\title{
IV. \\ Reports of Meetings of Commissions \\ Comptes Rendus des Seances des Commissions
}


Report of Meeting 1982 August 20

ACTING PRESIDENT: T Lederle

SECRETARIES: H Schwan

B D Ya11op

1. Organisation and Membership

The Vice-President $T$ Lederle reminded members that about a month ago he had informed members and consultants of the Commisston that he had taken over as Acting President since the President, MA Sinzi was unable to carry out his duties. He then put forward proposals for the officers and membership of the Commission; these were agreed as follows:

New Officers of the Commission for the next three years:

President: $\quad$ T Lederle

Vice-President: $\quad$ B L Morando

Organizing Committee: V K Abalakin, S Aoki, R L Duncombe, J H Lieske, A Orte, P K Seidelmann, M A Sinzi, G A Wilkins, B D Yallop

New nembers of the Commission: F Chollet, Y Kubo, N-y L1

New Consultants: $\quad \mathrm{H}-\mathrm{J}$ Felber, $\mathrm{K}$ G Steinert

Resignations from the Commission: J Kovalevsky, H G Walter

2. Working Group on Nutation

The Chairman said that the future of the Working Group on Nutation should be considered since its recommendations had now been adopted.

J H Lieske drew attention to J Vondrák's view that some significant planetary terms had been omitted. P X Seldelmann, the Chairman of the Working Group, said that the uncertainties in the nutation are insignificant in comparison with the relativistic effects which should be included in the reduction. E $M$ Standish suggested that it might be more useful to disband the Working Group on Nutation and create a new one on astronomical constants. $R$ L Duncombe agreed that the Working Group on Nutation had served a useful purpose and proposed that it should be discontinued; this was adopted with a vote of thanks to the retiring Chairman, $P$ Keidelmann.

\section{Bureau on Astronomical Ephemerides}

B L Morando, the Director of the International Information Bureau on Astronomical Ephemerides (BIIEA), asked the meeting to consider the possibility of closing down the, Bureau. The BIIEA is sponsored by COSPAR and supported by the IAU, but now the IAU is no longer supplying money, the Bureau has stopped supplying information and the users no longer enquire about the service. Summarizing the situation $R$ L Duncombe sald that the Bureau once met a real need but had now served its purpose; he proposed a vote of thanks to the retiring Chairman. 


\section{Transits of Mercury}

A paper on the results of observations on the transits of Mercury made at Belgrade in 1970 and 1973 was read by V Protitch-Benishek. The observations were recorded photographically and did not use the method of timing by visual contacts as in earlier transits. The method yielded a much better value for the solar parallax than before.

\section{Motion of Uranus}

A paper on the deviations of astrolabe observations of Uranus taken in Chile since 1975 was read by $F P$ Noël, who compared the observed rlght ascensions and declinations of Uranus directly with the Astronomical Ephemeris, the Astronomical Almanac and DE118. $R$ L Duncombe stressed the importance of continuing to make astrolabe observations of planets because they are subject to different types of error from transit circle observations.

\section{Radius of the Lunar Profile}

$P \mathrm{~K}$ Seldelmann proposed that a new value should be adopted for $k$, the ratio of the radius of the lunar profile to the Earth's radius (see Resolution 1), in calculations of lunar and solar ecllpses; this was necessary to prevent different almanacs using different values. The term "lunar radius" in the original draft was modifled to "radius of the lunar profile" and the resolution was then adopted without objection.

\section{Report of Joint Meeting of Commission 4, 7,8,19 and 31 on 1982 August 20}

Chairman: T Lederle

\section{HOMOGENIZATION OF COORDINATE SYSTEMS}

\section{Nutation}

P K Seldelmann, Chalrman of the Working Group on Nutation, discussed the developments after 1979 that led to the resolution on the 1980 IAU Theory of Nutation (see Resolution 2, and also Report for Commission 4, Section III and Appendix in Trans. IAU XVIIIA). He asked the meeting to endorse the adoption of the 1980 IAU Theory of Nutation which had been agreed by the majority of members of Commissions $4,7,8,19,24$ and 31 in separate postal votes. Resolution 2 was adopted unanimously.

\section{Report on the FK5}

W Fricke gave a report on the FK5. The specification of the FK5 was fixed by the 1976 IAU resolution on astronomical constants. The determination of equator and equinox of the FK5 was completed in 1981. The systematic corrections to the FK5 will be finished by December 1983, and individual star corrections for the FK5 will be ready by December 1985. Fricke sald that about 250 catalogues had been investigated involving 3400000 individual observations, and he gave a list of names of those who had made significant contributions to the determination of the FK5 equinox.

J Kovalevsky drew attention to the overlap between the MERIT campaign and the date of the introduction of the FK5 system and asked on what system w111 the astronomical observations be reduced durlng the campaign. G A wilkins replied that the procedure for the computation of star positions during the campaign will be decided by the MERIT Working Group on Standards. He hoped that the report will be 
distributed before the end of the year and final decisions will be taken at the second MERIT Workshop in May 1983.

P D Hemenway asked Fricke about the completeness of the final selection of faint FK5 stars. Fricke replied that there are about 50000 possible candidates with suffictent observational history and a good distribution in the sky. At most 5000 of these stars w111 be included in the FK5.

\section{Terrestrial Reference Frame}

I I Mueller read a paper by $S-y$ thu and himself on the effects of adopting new definitive precession, nutation and equinox corrections on the terrestrial reference frame. It was shown that the effect on polar motion is a diurnal periodic term with an amplitude increasing linear1y with time, whilst the effect on UT1 is a linear term. General principles were given to determine the effects of small rotations (such as precession, nutation or equinox corrections) of the frame of a Conventional Inertial Reference System (CIS) on the frame of the Conventional Terrestrial Reference System (CTS) in both stellar and non-stellar observations. Seven options to change the CTS to accommodate these sma1l rotations (corrections) were produced, one of which will have to be chosen. The last option, which requires no change in the origin of terrestrial Iongitudes or in UT1, is advocated. This option would be maintained by eventually referencing Greenwich Mean Sidereal Time to a fixed point on the equator, instead of the mean equinox of date, as is the current practice. Accommodating possible future changes in the astronomical nutation was also considered. The effects of differences which may exist between the various CTS's and CIS's (inherent In the various observational techniques) on Earth rotation parameters (ERP) and how these differences may be determined were mentioned. CTS differences are determined from observations made at the same site, whilst CIS differences are determined by comparing the ERP's obtained from the different techniques during the same period. The paper will be published in Bulletin Géodésique, 1982 .

J H Lieske disagreed with Mueller on the size of the effect on longltude for a non-stellar type of observation in his first option which was being investigated at JPL. The large $E_{0}$ term will not appear whilst the precession and equinox drift effects will largely cancel each other out. It is expected that the total effect from VLBI and radio tracking observations between the current system and the new system will be less than one metre. Mueller replied that geodetic appl1cations require better than one metre, and suggested that JPL is solving for the rotation of the frame and so is absorbing some of the effect.

4. Relationsh1p between GMST and UT1

$P$ K Seldelmann introduced a resolution on the expression for GMST at oh UT 1 (see Resolution 3) by drawing attention to the small changes from the resolution adopted in 1979. S Aoki suggested that the definition should give the relationship between Greenwich sidereal date and any instant of UT1, not merely Oh UT1. Seldelmann sald that this could be regarded as a direct extension of the original fundamental relation. J H Lieske opposed the resolution on the grounds that the $\mathrm{T}^{2}$ and $\mathrm{T}^{3}$ terms served no physical purpose. Furthermore if UTl is deduced from a transit observation 1 ts value will depend upon whether the reduction is made In the FK4 or the FK5 system. Seldelmann replied that the resolution adopted in 1979 included numbers which were based on preliminary values of $E$ and $\dot{E}$, therefore the equation had to be changed. The correction for the quadratic and higher terms were introduced to correct the effect of Newcomb's error of 1890 and so the equation has a sound basis for future investigations. $S-h$ Ye read a paper by $B-x X u, J-p$ Ren and $Z-s$ Sun on a new definttion of Universal Time where UTl is directly proportional to the sidereal time, which is a more natural 
and a much easter concept. T Lederle commented that others have thought on simflar 1ines. B Guinot said he had not changed his mind about the use of the non-rotating origin, but it is not possible to introduce a completely new concept in so short a time; we must adopt the proposed GMST/UTI relation now.

Seidelmann agreed to Muller's suggestion that the words "with respect to the stellar reference frame" be added to the resolution in the first sentence after "there is no change in either value or rate of UT1", and pointed out that Mueller's comments indicated that there was still room for study in these areas.

The resolution was adopted with some abstentions.

\section{Sign Convention for Terrestrial Longitude}

G A Wilkins proposed a resolution that the sign convention east longitude is positive should be used for terrestrial longltude (see Resolution 4). He drew attention to the many circumstances in which this convention is already in use and to the difficulties caused by the use of the opposite convention in some astronomical almanacs and publications. S V Débarbat asked if 1 t was also necessary to adopt a sign convention for azimuth. Wilkins replied that this should be considered as a separate issue and that the resolution should not be extended. $T$ van Flandern pointed out the inconsistancy that will still exist between planetographic and planetocentric longitudes on the Earth. The resolution was adopted by an overwhelming majority.

Report of Joint Meeting of Commissions 4, 19 and 31 on 1982 August 20

Chairman: T Lederle

ASTRONOMICAL CONSTANTS AND EPHEMERIDES

1. New Ephemerides

$E M$ Standish described the work done at JPL in collaboration with members of USNO in producing the DE200/LE200 ephemerides. They w111 form the basis of the Astronomical Almanac from 1984 onwards. The observations used to construct the ephemerides were lunar laser ranging to $30 \mathrm{~cm}$ precision, Earth-Mars ranging $(10 \mathrm{~m})$, and planetary radar $(1-2 \mathrm{~km})$, together with optical transits and astrometry ( $350 \mathrm{~km}$, or $100 \mathrm{~km}$ at $1 \mathrm{au}$ ) to the the ephemeris to the FK4 equinox. JPL has produced software for distribution; one routine will provide the ephemeris of a body or bodies and the other will provide a complete list of the constants used. The fundamental ephemeris was produced by fitting a simultaneous integration of all bodies, including the Moon to the observations; for nutation the 1980 IAU Theory of Nutation was used. The or1gin of the DE200/LE200 reference frame 1s the mean equator and dynamical equinox of $\mathrm{J} 2000.0$ which is almost identical to the origin of the FK5. The accuracy was 0:05 or better for the inner planets and $0: 2$ to $1: 0$ for the outer planets.

$P \mathrm{~K}$ Seldelmann discussed the work done at USNO on the new ephemerides and showed comparisons between transit observations at USNO and DE200/LE200. There were large waves in declination for Saturn and the fit for the outer planets ran off. It was found impossible to fit Uranus observations to the ephemeris before 1900 without the current ephemeris going of $f$. It was also found necessary to modify some of the 1976 IAU astronomical constants in order to obtain the best fit.

B L Morando described the work done at the Bureau des Longitudes on new theories for the Moon and planets. New analytical theorles had been developed and 
had been fitted to DE200/LE200. They have used the IAU (1976) System of Astronomical Constants. Tapes are available containing the analytical series. The basis of the method w1ll be published in Astron. \& Astrophys. The Connaissance des Temps will use the ephemerides based on the new theories and will give the elements of the orbits for all the bodies.

\section{Discussion on Resolutions}

$P \mathrm{~K}$ Seidelmann proposed a resolution that considering that it is planned to introduce the IAU (1976) System of Astronomical Constants, the 1980 IAU Theory of Nutation, the equinox of the FK5 and Dynamical Time, in 1984 January 1 , it is recommended that the ephemerides published in the international and national ephemerides be based on the above resolutions and the avallable observational data to the extent possible to provide the best prediction ephemerides possible.

B L Morando and others raised objections to the resolution, particularly on the grounds that its meaning was not sufficlently precise, and so Seldelmann withdrew it.

Seldelmann then put forward a second resolution which recommended that some of the values of the IAU (1976) System of Astronomical Constants should be revised to form the IAU (1982) System of Astronomical Constants. He pointed out that it had been necessary to use the new values in order to obtain an optimum fit of the new fundamental ephemerides DE200/LE200 to new high precision observational data. Morando considered that the IAU system should not be changed until a working group had considered in detall the evidence and the arguments for change; the 1976 values had been used in the new ephemerides for the Connaissance des Temps. Aok1 pointed out that the 1976 values would also be used for the Japanese Ephemeris. Lederle considered that such a resolution should be submitted for discussion in advance of the Assembly. H F Fliegel drew attention to the analogy with the change In the adopted nutation theory; the Commissions had refused to adopt a new theory in 1979 but had later found it desirable to do so. Duncombe supported the view of Lederle and proposed that the discussion of the resolution should be suspended. Th1s was agreed by a vote of 12 in favour, with 2 against.

Seldelmann then proposed a third resolution which was intended to confirm the general practice that the dynamical equinox be defined as the intersection of the mean celestial equator with the smoothly moving ecliptic plane at which the Sun's declination is changing from South to North. He pointed out that it is also posstble to determine the position of the equinox by treating the ecliptic as a fixed plane and that leads to a difference of $0: 1$ in position. After some discussion 1t was agreed not to vote on the resolution, even though there were no objections to the wording, since it was not a new definition or practice.

\section{RESOLUTIONS}

Resolution 1 of Commission 4 on the value of the ratio of the radius of the Iunar profile to the Earth's radius.

Commission 4 considering

that it 1s planned to introduce the IAU (1976) System of Astronomical Constants, the 1980 IAU Theory of Nutation, and the equinox of the FK5 on 1984 January 1,

recommends

that in the calculation of lunar and solar eclipses, there be a single value for $k$, the ratio of the radius of the lunar profile to the Earth's radius, and that it correspond to the mean radius of Watts' datum as determined by observation of occulta- 
tions and to the adopted radius of the Earth. The value is $k=0.2725076$.

Resolution 2 of Commissions 4,7,8,19 and 31 on the 1980 IAU Theory of Nutation.

Commissions $4,7,8,19$ and 31 recognizing

that since the Seventeenth General Assembly of the IAU, Commissions 4, 7, 8, 19 and 31 have adopted the 1980 IAU Theory of Nutation in place of the 1979 IAU Theory of Nutation,

endorse

the adoption of the 1980 IAU Theory of Nutation.

Resolution 3 of Commissions 4,19 and 31 on the expression for GMST at oh UT1.

This resolution $1 \mathrm{~s}$ an amendment to the provisional part of Resolution 3 of Comm1ssions 4, 19 and 31 made at the Seventeenth General Assembly of the IAU.

Commissions 4,19 and 31 considering

that 1t 1s planned to introduce the IAU (1976) System of Astronomical Constants, the 1980 IAU Theory of Nutation, and the equinox of the FK5 on 1984 January 1 ,

recommend

that (a) the relationship between mean sidereal time and UTl be modified so that there is no change in either value or rate of UTl with respect to the stellar reference system, due to a correction to the zero point of right ascensions of the FK4 and to a correction for the motion of the zero point to be introduced in FK5,

(b) the new expression for Greenwich mean sidereal time of oh UTl be

$$
\begin{aligned}
\text { GMST of } 0^{\mathrm{h}} \text { UT1 }= & 6^{\mathrm{h}} 41^{\mathrm{m}} 50.54841+8640184^{\mathrm{S}} 812866 \mathrm{~T} \\
& +0.093104 \mathrm{~T}_{\mathrm{u}}{ }^{2}-6.2 \times 10^{-6} \mathrm{~T}_{\mathrm{u}}
\end{aligned}
$$

where $T_{u}$ is the number of Julian centuries of 36525 days of Universal Time elapsed since 2000 January $1,12^{\text {h }}$ UT l (JD 2451545.0 ).

Resolution 4 of Commissions 4, 19 and 31 on the sign convention for terrestrial

longitude.

Commissions 4,19 and 31 noting

that the International Meridian Conference held in Washington in October 1884 adopted a resolution that from the meridian of the Observatory of Greenwich "longitude shall be counted in two directions up to 180 degrees, east longitude being plus and west longitude minus",

noting

that there is an increasing interaction between astronomy and geodesy in which field the sign convention "east longltude is positive" is in common use, recommend

that as soon as practicable all national ephemerides and other astronomical publications adopt the convention that terrestrial longitude be measured positively to the east. 
Report of Meetings, 18, 21, and 23 August 1982

I. WORKING GROUP ON ASTRONOMICAL DATA

CHAIRMAN: B. Hauck.

The first part of the meeting was devoted to business. The Chairman reported on the activity of the Working Group during the period 1979-1982. The main activity was concentrated on trying to centralize information concerning astronomical plate vaults (see IAU Inf.Bul1.48, June 1982). The Working Group also prepared a resolution concerning FITS. The Chairman attended the assembly of CODATA at Kyoto in 1980 as IAU Delegate.

Drs. Mead and Terashita were elected as new members of the Organizing Committee, the membership of which is otherwise unchanged. Chairman and Vice-Chairman will remain in office. It was decided to modify the structure of the W.G. by removing the notion of subgroup, and by no longer considering "Nomenclature" to be relevant to this Working Group. G.Wilkins then presented the finalized "Guide to the Presentation of Astronomical Data" (CODATA Bulletin 46, 1982). This Guide contains general recommendations to editors, referees, and authors on the reporting of numerical data obtained from astronomical observations. Copies may be ordered from the CODATA Secretariat, 51 Bd de Montmorency, 75016 Paris. B. Hauck reported on the plate vaults and was able to announce that two observatories had already sent the copies of their files to the Stellar Data Centre at Strasbourg.

The scientific part of the meeting was devoted to "Transfer of Astronomical Data". P.Grosbol reported on the Flexible Image Transport System (FITS), the purpose of which is to simplify the problems involved in the transfer of arrays of "image" data between different observatories, and different computer systems in particular. Data networks are also important for the transfer of data. Presentations on such networks in the United Kingdom, Italy, and France were prepared, respectively, by K. Tritton (read by G.Wilkins), G.Sedmak (read by S.Catalano), and F. Ochsenbein.

The composition of the Working Group as of 1982/83 is:

Chal rman: B. Hauck Vice-Chairman: G. Westerhout

Organizing Committee: W.P.Bidelman, O.G.Dluzhnevskaya, R. Duncombe, W. Heintz, C. Jaschek, T.Lederle, J.Mead, Y.Terashita, G.Wilkins.

Members: J.F.Dolan, R.S.Dixon, P.Grosbo1, A. Heck, P.Lantos, G.Lynga, J.Nishimura, F. Ochsenbein, L. E. Pasinetti, A.G.D.Philip, J. Shakeshaft, F. Spite, K. Tritton, P.T.Wallace, W.Warren.

\section{WORKING GROUP ON EDITORIAL POLICIES}

CHAIRMAN: S. Mitton.

The Chalrman presented the draft of the Style Manual for the IAU Astronomers' Handbook, and it was discussed by the participants of the meeting. Some amendments were suggested, and the proposed changes were agreed on after discussion. 
The document will be circulated, prior to its final publication, to editors of astronomical periodicals, in order to invite their comments also. The Chairman agreed to present a copy to the IAU EC immediately for comments. The Working Group believes that its continuing existence is not necessary once it has discharged the duty of revising the Handbook.

\section{WORKING GROUP ON CLASSIFICATION SYSTEMS AND INFORMATION RETRIEVAL}

CHAIRMAN: P. Lantos.

While this W.G. presented its report at the Full Commission Meeting, a special meeting was convened to discuss controlled vocabularies, their compatibility and desirable extent, and the feasibility of allowing composite, derivative, or free key words. L. Schmadel and P. Lantos will be in contact in order to examine currently used or proposed vocabularies in these respects. (From notes by W.Heintz.)

\section{SPECIAL WORKING GROUP ON DESIGNATIONS}

CHAIRMAN: C. Jaschek. SECRETARY: W. Heintz.

W.P. Bidelman has chaired the W.G. during the past term, and has completed his work with a comprehensive report; he was unable to attend the Patras conference. The business now at hand of the W.G. will be to implement the projects approved in Montreal in 1979, and to reach more impact of its recommendations, particularly with the help of scientific editors of journals. In pursuance of previous efforts, object designations should reach a degree of uniformity that permits rellable retrieval of material from all modes of data storage.

A related matter are the catalog codes, on which F. Spite reported. The draft of the standard list (Fernandez, Lortet, and Spite) was presented. (Note: A revised version was issued after the conference, in September 1982.) Completion of the work and its publication (about $120 \mathrm{pp}$.) are expected shortly. The W.G. calls attention of regional organisations to this catalog, and invites its publicizing in local newsletters.

Designation practices in various object categories will be the subject of a booklet to be published by J.Mead; in her absence this was reported on by Parsons. In view of new methods of discovery, Pecker advised that first publications should reference earlier catalogs relating to the objects in question. The Index of Double Stars (IDS) was cited as an example of a desirable compilation format. The recommendations on designations from past IAU Assemblies - a substantial though not always consistent list that has accrued - should be screened in order to arrive at standards which the periodicals will accept. Commission 37 reported 1 ts agreement to use the equator 1950 for cluster center coordinates.

Grosbol discussed the presentation of catalogs, especially in view of their exchange by magnetic tape. He advised the creation of an ad-hoc group (with Harten, Ochsenbein, Tritton, and Warren) which will study the matter, and expects to formulate appropriate recommendations.

Jaschek proposed to Commission 5 the following actions: That the mandate of the Working Group on Designations be extended for another period; that the W.G. contact journals in order to emphasize conformity with the recommendations approved at Montreal 1979; and that further input be solicited from IAU Commission presidents concerning unified practices of designation. 
The 1980 Report of the W.G. as written by Bidelman, and published in Bul1. CDS 18, 41 will go to Commission 5 and to the IAU Executive Committee for adoption. Further work on designations is expected to be published in the Bulletins of CDS (Strasbourg, France) and of NSSDC at Goddard Center (Greenbelt, USA).

\section{FULL COMMISSION MEETING (23 August 1982)}

PRESIDENT: W. D. Heintz.

SECRETARY: R. J. Davis.

Attendance: 23. Agenda: (1) Reports, (2) Business, (3) Resoluttons and recommendations, (4) Papers, (5) Announcements.

(1) The Chalrman recalled the recent history of expanding coordinative responsibilities of the Commission, which includes the secondary processing of scientiflc material from the time it leaves the authors' desks, to its retrieval by 1 ibrarians, abstracting and data services, and their clientele. The restructuring of the Commission and its Working Groups, accompanied by a doubling of membership, has taken place largely under the leadership of Past President J.-C.Pecker. A vote of thanks to him was applauded.

Some concern had arisen that the activities might be too broad and diversified, and better served by two commissions. However, the interfacing nature of coordinating activities (related to computers, standardisation, retrieval procedures, and the corresponding contacts with other Commissions) had already in Montreal supported the general consensus that this administrative unity should remain intact.

One successful result of this integration was the IAU Colloquium 64 (in Strasbourg, July 1981). C. Jaschek is credited with initiating and organizing the conference (together with his colleagues), and also with efficient editing work so that the volume of the Proceedings appeared already in May 1982.

The Chalrman then reported on the ICSU Abstracting Board, which is in a process of reorganisation toward its expected goals in the $1980^{\prime} \mathrm{s}$. Relevant recommendations will be formulated (with IAU participation) at the Executive Committee meeting in December 1982. It is recommended to the Commission and to the IAU EC that the membership in $A B$ be maintained at least until its new structure has emerged. The volume of abstracting work, the technology employed, and the sales revenues required to maintain good abstracting services, all of this renders $A B$ a meeting of experienced speclalists, working in cooperation with - and advised by - ICSU Unions but not under their exclusive contro1. The 60-year-old Commission 5 of IAU always has been a forum of interfacing on bibliographical matters; most other Unions do not have a similar body. One concern is the growing habit to treat astronomy and astrophysics as part of physics. This results in a bibliographic processing which simply does not meet the needs of the astronomical research community.

Some amusing stir has been provided by private "agencles" trying to sell star names for profit. W1thin ICSU, and thus under UNESCO auspices, the IAU and its authorized committees are in charge of nomenclature of celestial objects. Although not subject to copyright, the names have to comply with documentation standards to serve as useful identiflers (the Spite et al master list of codes, for example). Having exposed the "star registries" as rip-offs without sclentific backing, any further pressing-the-point by IAU would be a waste of time.

G.Wilkins, Vice-President, spoke about the documents on standards for the publication of data. He suggested a recommendation advocating compliance of astronomers with the "Guide" (CODATA Bull. 46), after its pending endorsement by the IAU Executive Committee. 
Following a suggestion by Jaschek at the Strasbourg meeting, a sma11 Working Group on a glossary of terms was set up. One portion, that on Computing, has so far been completed. Wilkins suggests that the work of this group continue.

The present document on subject classification for astronomy was 1ssued in 1975 and is now in need of revision. A group working on the revision is chatred by Wilkins. Unfortunately for the input, this document is not widely known to astronomers. It can be bought from the British Standards Institute, 2 Park St., Iondon W1A 2B5, and has the catalog number BS1000(52):1977. Of interest to astronomers is also the FID publication No. 483.

B.Hauck, IAU delegate to CODATA, referred to the reports in CDS Bul1.23 (1982) and in CODATA Bul1.36, chap. 3 of which is devoted to astronomy. Astronomers may benefit greatly by keeping track of CODATA activity. Jaschek suggested that CODATA sponsor a school for young astronomers as an introduction to data techniques.

P.Lantos, for the W.G. on Classification Systems, had a progress report on the IAU vocabulary and the key-word list. The vocabulary, using a number of standard sources, was originally created in English; a French version has now been evaluated by a number of French scientists. About 1000 entries are in the key-word list presently. Pecker observed the importance of uniform documentation, and of the responsibility of the authors in selecting key words; he suggested a deadline for completion of the vocabulary project. Heintz felt that the key-words should be agreed on and finalized first, and that IAU be asked to maintain the offer of publication expenses for the vocabulary.

S. Mitton summarized the revision of the Style Manual for the Astronomers Handbook, which is undergoing review now. Publication in IAU Trans. XVIII B is expected. Copies will be sent to the editors of the main journals. It is to be hoped that the IAU Manual will receive more attention.

(2) The business section of the agenda was opened by J.-C.Pecker in the chair. He recalled the Rules of the Commission, and its status as Committee of the IAU Executive Committee. For that reason the officers normally serve two terms. Pecker proposed the re-election of Heintz as President, Wilkins as Vice-President, and Schmadel as continuing member of the Organising Committee. This was approved by the vote of the Commission. Resuming the chair, Heintz led a discussion on the WorkingGroup structure of the Commission.

Hauck and Lantos were confirmed as continuing Chairmen of their respective Working Groups, and as ex officio members of the Organising Comittee. Mitton's group was discontinued, following completion of work on the new Manual, and the Commission appreciated Mitton's effort with applause.

The Special Working Group (Task Force) on Designations had been chaired by W. Bidelman, who indicated by letter to the Commission that he thinks that the bulk of the work has been finished. However, from the tasks assigned to that Group some incomplete segments of work ensue, viz.: To finalize the reports in discussion with other IAU Commissions (which may require some more time on part of the Commissions), in order to unify the practices of object designations, and to get the results to the status of IAU recommendations, published, and put into practice. It would be pointless to make resolutions unless there were sufficient inclination to enforce them. The Commission resolved: (a) To adopt the Bidelman Report as printed in CDS Bull. 18 and to express great appreciation to Bidelman for his work; (b) To continue and to promote the group to a full Working Group (Title: Designatlons); To install Jaschek as Chairman of the W. G., with H. Dickel, M.Lortet, J.Mead, J.-C.Mermilliod, and F. Spite as other nominated members.

There were requests for the appointment of two new Task Forces. 
The Special Group on Standardisation of Exchange of Astronomical Data, to be chaired by P.Grosbol, was approved as proposed in the preceding meeting on designations. Following a discussion at the Abstracting Board meeting in Pine Mountain in 1981, a Special Group to explore guidelines for Abstracts in Astronomy was suggested. This was approved, and the Cairman asked L. Schmadel to temporarily chair this Group and to prepare a slate of proposed members.

The Working-Group structure for 1982-85 is thus: Astronomical Data (Hauck); Classification Systems (Lantos); Designations (Jaschek); Standardisation of Data Exchange (Special Group; Grosbol); Guidelines for Abstracts (Sp.Group; Schmadel).

For two vacancies on the Organising Committee, three nominations were at hand. C. Jaschek having already become a new ex officio member of the 0. . , the Commission voted O.B.Dluzhnevskaya and C.E. Worley into office.

The Chairman paid tribute to three deceased members: J.0.Fleckenstein, J. Wempe and H.Hirose. Two members and several consulting members have resigned.

Six new members were admitted: S.Nishimura, K.P.Tritton, P.T.Wallace, P.Wayman, I. Heinrich, F. Ochsenbein (the latter two pending approval of proposed IAU membership). Reconfirmed consultant members: Bérardini, Berthélot, Collins, Dudley, Grassi, Guidon1, Laforge, Laloe, Martin, Schiminovich; new consultants: Corbin and de Narbonne. The Commission file now lists 59 members and 12 consultants.

(3) The Commission acted affirmatively on two Resolutions (see Report of General Assembly, 26 August) and endorsed several papers to be forwarded to the IAU General Secretary, including Wilkins's Guide and Mitton's Manual. The Chairman reported that no motion concerning the Rules of the Commission had been received.

(4) B. Corbin (U.S. Naval Observatory Librarian, and member of the Special Libraries' Association) reported on a compilation list of astronomical serials from their beginning and of astronomical books before 1800, including data on where available. In the Amer. Astr. Society a section has been established for the preservation of documents and instruments; microfilming the old papers is intended. In order to avold dupllcation, information is requested if work of this kind is already obtainable elsewhere. Librarians deem the Astr. Astrophys. Abstracts a superb and important publication, and they would much appreciate to have it also in machine-readable form. Schmadel responded that the matter is under negotiation. Wilkins mentioned the archive preservation program of the Royal Greenwich Observatory. He and Heintz recommended to reactivate the initiative and input by librarians in the Commission and the IAU, encouraging Dudley (Greenwich) and Corbin to further contacts.

G. Grassi Conti (Rome Observatory Library) had a report (read by M. Smyth) on the catalog of 17 th-century European books; the 1ist will perhaps be merged with the 1516 th-century catalog currently being updated. Since response to the request for information was not quite complete, support by the Commission was desired. Libraries with pre-1700 holdings are urged to aid in the extensive efforts on an important compilation by contacting Grassi.

(5) The new edition of the Multilingual Dictionary by J.Kleczek is finished, except for some final checking. Since it was found not feasible to include Chinese and Japanese presently, and the work should go to press shortly, these languages should be incorporated in a later supplement. - Other announcements concerned new databases and reference papers.

The Commission voted to co-sponsor a symposium contemplated for 1984 by the President of Commission 42, with support by Comm.29, under the title "Calibration in Astronomy". In view of the (Intentionally) broad scope of the subject, the participation by Commision 5 was invited. - The Commission meeting then adjourned to 1985. 
COMMISSION 6: ASTRONOMICAL TELEGRAMS (TELEGRAMMES ASTRCNOMIQUES)

Report of Meeting, 20 August 1982

PRESIDENT: J Hers

SECRETARY: B.G. Marsden

After having been welcomed by the President, members and guests were asked to stand in memory of Dr. E. Buchar, a former President of the Commission, who died in 1979. This was followed by the confirmation of nominations for officers of the Commission for the period 1982-1985: President, M.P. Candy; Vice President, A. Mrkos. No changes were proposed in the general membership of the Commission.

The President reported that, in view of constantly rising costs, it had again been necessary to approach the Executive Committee regarding an increase in the IAU subvention for the Central Bureau for Astronomical Telegrams. This resulted in an increased amount of S Fr. 10000 for the period 1982-1985 being proposed in the Draft Budget, which was subsequently approved by the Finance Committee. While this represents less than 5 percent of the actual running costs of the Bureau, it nevertheless remains tangible proof of the high regard in which the Bureau is held by the Executive Committee, and a letter to this effect received from the General Secretary was noted with appreciation.

Dr. Marsden, in his capacity as Director of the Central Bureau for Astronomical Telegrams, briefly discussed the report on the Bureau's activities during the period 1979-1982, as published in Transactions XVIIIA. He noted that all Commission members present at the Meeting were, without exception, also members of Commission 20, which might give the impression that Commission 6 was mainly a subsection of Commission 20 . This was by no means the case, and to stress this fact a special effort had been made some years previously to include representatives from other Commissions. However, these had taken little or no part in Commission 6 activities. The reason might be that rapid dissemination of astronomical data still remained, as in the past, mainly of interest to observers of minor planets, comets and related objects, while it was evident that workers in other fields were entirely satisfied with the operation of the Bureau.

Dr. Marsden explained that the IAU Circulars were currently being produced by him with the aid of two IBM electric typewriters, one in the office and one at home, which made it possible' for urgent Circulars to be sent out at very short notice. A more modern approach might make use of a microcomputer plus word processor, enabling the work to be done more quickly, and maintaining a uniformly high standard. This would have the added advantage that the material would be in machine readable form, and might thus be made directly available to users in that form. Postal services had deteriorated tremendously during the preceding few years (a brief enquiry among members present showed that the Circulars seldom took less than 5 days, and not infrequently took more than 20 days to reach their destination) and any method to speed up the process deserved serious consideration.

Questions raised in the ensuing discussion included the probable frequency with which users might dial for information (daily, say, or weekly?) and how this service was to be paid for. It was pointed out that a large part of the income of the Bureau was derived from subscriptions to the Circulars, and this was an income which the Bureau could not afford to lose. On the other hand, users outside the U.S.A. might find it difficult and expensive, perhaps even impossible, to make use of such a service, and the Circulars would doubtless have to continue 
to exist in their present form for many years to come. Nevertheless, it was agreed by the meeting that the modern way would be electronic rather than by means of pieces of paper, and it was agreed that investigations should continue.

This was followed by a report by Dr. Marsden on a meeting held on August 18 between representatives of Commission 6 (Hers and Marsden), Commission 20, and the Working Group on Planetary System Nomenclature (WGPSN). This meeting had been called, and Commission 6 had become involved in it, because it had become evident that there was a tendency to allocate designations and names to objects in the Solar System without due regard to existing well-established practices. These practices, based on long experience, and designed to reduce the possibility of errors and misidentifications to a minimum, had never received the formal endorsement of the IAU, and the Bureau was experiencing increasing difficulty in having to deal with incorrectly designated objects. The meeting had therefore drawn up a table, agreed to by all concerned, which allocated responsibility for all the various steps, from initial temporary designation to the final naming of planets, satellites, rings and comets. According to this, Commission 6 , through the Central Bureau for Astronomical Telegrams, remains responsible for the temporary designation of principal planets, satellites, rings and comets (but not minor planets), and for the naming of comets.

The Meeting ended by approving the proposed allocation of responsibility, which was to be submitted to the Executive Committee for formal IAU approval. 
COMMSSION 7: CELESTIAL MECHANICS (MÉCANIQUE CÉLESTE)

Report of Meetings on August 19, 1982

PRESIDENT: Y. Koza1

SECRETARY: H. Kinoshita

\section{Business Session}

\section{OFFICERS OF THE COMMISSION}

Recommendation for the following list was moved and unanimously approved:

President: J. Kovalevsky, the retiring Vice-President, Vice-President: V.A. Brumberg

Organizing Committee: E.P. Aksenov, J. Chapront, S. Ferraz-Me11o,

J. HadjIdemetrlou, J. Henrard, W. Jefferys, Y. Kozai,

H. Scholl, P.K. Seidelmann, A. Sinclair

MEMBERSHIP OF THE COMMISSION

The president reported that when China became a member country of the IAU the following three Chinese astronomers were approved as new members of the Commission: Tong $\mathrm{Fu}, \mathrm{Yi}$ Zhao Hua and Zhao Xian $\mathrm{Zi}$.

The following members of the IAU were proposed as new members of the Commission 7 and approved:

R. Meire and M.B.Moons(Belgium), W. Sessin(Brasil), B.B. Baghos, Y.E. Helali and M.Y. Tawadrous(Egypt), Z. Sima and M. Yidlichovský(Czechoslovakia), D. Benest and F. Mignard(France), A.G. Mavraganis and Ch. Varvoglis (Greece), B. Erdi(Hungary), K.B Bhatnagar(India), A. Milani(Italy), J. de Orus (Spain), D.B. Taylor(UK), and P.E. Elyasberg, N.V. Emelianov, V.A. Izvekov, B.N. Noskov, B.S. Novselov and S.K. Tatevian(USSR).

As S. B Bhme left the commission the total number of the members is now 148 .

CONSULTANTS

The following 6 persons were approved as consultants of the commission for the coming three years and approved:

V.A. Arnold, R.R. Allan, V.R. Bond, C.J. Brookes, J.K. Moser and J. Boels.

\section{$\underline{\text { Scientific Session }}$}

The five review papers were presented between 10:00 and 13:00 with much discussions at the scientific session.

1) Planetary Theories ( J. Kovalevsky)

In the present days, no group has endeavoured to cope simultaneously with the three tasks that have to be performed in order to construct a planetary theory: collect and reduce observations, obtain a theoretical representation of a set of solutions in the vicinity of the actual motions and compute the best fitting orbits. Two approaches have permitted to skip at least one of these tasks. Numerical integrations - the most recent being D.E. 200 by E.M. Standish(JPL) - skip the second task. In the approach by Bureau des Longitudes(P. Bretagnon, J. Chapront and J.L. Simon), an analytical theory has been derived for the 8 major planets, and the fit is made with D.E. 200 that is used as a kind of a collection of normal points. 
Two methods have been applied. In the iterative method, the solution of order $n-1$ is substituted in the right hand members of the equations and after integration and fitting of the pre-established constants of integrations, one obtains the $n$-th approximation. In the ordered solution, equations are developed in powers of the varlations of the elements, so that there is no subsitution, but it is necessary to compute a great number of partial derivatives. The final solution in Bureau des Longitudes was obtained in Poisson series of time to the third order of masses for the 4 inner planets by the ordered method and to the sixth order of masses for the outer planets by a combination of both methods - checked by the iterative method. After fitting to D.E. 200, maximum errors for 100 years are $+0 . " 002$ for Mercury, $\pm 0 . " 005$ for Venus and Earth, $\pm 0 . " 02$ for Mars and Uranus and $\pm 0 . " 01$ for Jupiter, $\bar{S}$ aturn and Neptune.

But it is very necessary to Improve also the general theories where the time is not allowed to appear outside trigonometric arguments. It is shown as an example, that the number of arguments must be larger by at least 2 or 3 orders of magnitude. The best available general theories for all planets present discrepancies of the order of $1 / 1000$ with Cohen and Desterwinter numerical integration over 700000 years. It is believed that one can do much better and that more work should be done in improving general planetary theories.

\section{2) Analytical Lunar Theories (H. Kinoshita)}

The accuracy of recent analytical theories has increased considerably during the last decade due to the requirement of lunar laser ranging observations and due to the rapid increase of capability of handling mathematical formulas by computer. Recent lunar theories are throughly investigated by different authors and various perturbation methods and have superseded Brown's theory. Recent analytical lunar solutions are divided into the following four categories according to the treatment of coefficients of Fourier series, by which solutions are expressed: analytical (or Iiteral) solution, partly analytical solution, semi-numerical solution, and semi-analytical solution. Advantages and disadvantages of each type of solution were briefly discussed. The precision of each solution was also discussed with use of comparison with numerical integration. ELP developed by M. Chapront-Touze and $\mathrm{J}$. Chapront, one of these solutions, is a complete solution in the sense that it includes all perturbations acting on the Moon except the effect due to non-rigidity of the Moon. The accuracy of solution of main problem in ELP is about a few centimeters and the accuracy of planetary perturbation in ELP is about a few meters over one hundred years. In order for an analytical solution to be compatible with the accuracy of lunar laser observations, we have to 1) increase precision of planetary perturbations(especially direct perturbations due to Venus and complete second-order planetary perturbations) and 2) investigate coupling effect among various perturbations (especially coupling between $J_{2}$ perturbation and planetary perturbation) and 3) increase precision of external theories(planetary theory, physical libration of the Moon, etc).

\section{3) Planetary Rings (J.A. Burns)}

The discovering of the rings about Jupiter and Uranus, plus the vast growth in our knowledge of Saturn's rings, has allowed substantial progress to be made in our understanding of their dynamical evolution. This talk summarizes the physical properties of the three ring systems and discusses the gross evolution(flattening, collisional spreading, location within the Roche 1imit) of unbound rings. It then describes how the lifetimes of small grains can be limited by dynamical processes (plasma drag and Poynting-Robertson effect) or by erosion(spattering and micrometeoroid collision); micron-sized grains, such as found in Juplter's ring and Saturn's E-ring, must be continually regenerated. Satellites have profound effects on ring structure: according to the mechanism proposed by Goldreich and Tremaine, satellites can repel disks of ring material and initiate spiral density waves at resonant loca- 
tions. About twenty-five spiral density waves have been identified in Saturn's rings with two of them bending waves.

Satellites also confine Saturn's F-ring and probably the Uranian rings; local short-time perturbations may produce the kinks and clumps in the F-ring while its bending is as yet unexplained. While our understanding of planetary rings has developed substantially over the last dcades, many questions remain unanswered.

4) Peroidic orbits in Dynamical Systems and Their Stability (G. Contopoulos)

When a family of stable orbits becomes unstable, it "inherits" its stability to a new family bifurcating from it. In general we have an infinity of successive "pichfork bifurcations" leading to an infinity of unstable families and a large degree of stochasticity. The ratio of successive intervals between bifurcations tends to the unfversal number 8.72 (Feigenbaum, Eckman etc). But we may have infinite bifurcations of the same family, which becomes successively stable and unstable. The corresponding ratio depends on the dynamical system and is not universal. We have also found a case of a Feigenbaum sequence followed by an inverse Feigenbaum sequence, leading back to a single stable family.

Another mechanism leading to large stochasticity is the breaking of a family to an infinity of families of periodic orbits separated by gaps. The extent of trapping around each family gives the non-linear stability of this family. We noticed that beyond each gap the central family of periodic orbits "inherits" its associated tube orbits to the next family.

\section{5) Dynamical Evolutions of Satellite Orbits (Y. Kozai)}

In the solar system there are many pairs of satellites, for which commensurable relations of the mean motions hold. Recently Voyager spacecrafts discovered several other commensurable pairs in Saturnian system. Why such commensurable relations, namely resonant configurations, are dominant in satellite systems? It is hardly believed that such relations have been dominant from the beginning of the solar system, and, therefore, some kinds of dynamical evolution processes should have slowly driven the systems in such resonant configurations. The most important factor to have driven the orbits is believed to be, for most of the cases, due to tidal dissipations in planets and satellites.

The tidal dissipations in the satellites tend to reduce the eccentricities even after the synchronization of the orbital motion with the rotational motion is realized. However, there are satellites, for which the eccentricity cannot be reduced to zero because of the forced eccentricity due to the resonant perturbations. For such pairs the tidal dissipation never stops and has produced volcanic activities in Io, one of Galilean satelittes.

Martian satellites are likely to be composed of carbonaceous chondrites, and the secular accelerations observed in the mean longitude of Phobos are due to the tidal dissipations. It is believed that Phobos was captured by Mars and come to the present orbit due to the tidal dissipation. 
COMMISSION 8: POSITIONAL ASTRONOMY

(ASTRONOMIE DE POSITION)

Report of Meetings of $19,20,21,23$, and 25 August 1932

PRESIDENT: E Høg

SECRETARIES: B L Klock, $T$ E Corbin

CONTENT

I. Commission Business

a) Constitution of the commission

b) Working Groups

c) Resolutions

d) Commission Rules

e) 1980 IAU Theory of Nutation approved

f) SRS

g) Symposium on Astrometric Techniques

II. Instrumentation

III. Stellar Reference Frame

IV. Extragalactic Reference Frame

I. COMMISSION BUSINESS (19 August)

a) Constitution of the Commission

President: J A Hughes Vice-President: Y Requieme

Organizing Committee: G Billaud, G Carrasco, W Fricke, VS Gubanov, EHøg, B L Klock, J A Lopez, Luo Ding-jiang, M Miyamoto, L V Morrison,

I Nikoloff, G Teleki, Y S Yatskiv

Deceased Member: $\mathrm{K} N$ Tavastsherna

Retired: none

New Members: L Helmer (DK), I Stange (GDR), R Bien, S Röser (GFR), L N Mavridis (Greece), M Yoshizawa (Japan), I Lindegren (Sweden), $R$ I Branham, P D Hemenway, R Stone (USA), V A Fomin, Yu L Kokurin (USSR)

Consultants: C Fabricius (DK), K C Blackwell (UK), J L Fanselow (USA), V I Orelskaya (USSR), M Dachich, I Pakvor (Yug)

Numerical Strength (with previous values in brackets) 15 (13) Officers, 103 (99) Members, 6 (9) Consultants, Total 124 (121) 
b) Working Groups

\section{Study Group on Astronomical Refraction}

Chairman: G Teleki. Members: W J Altenhoff (Bonn), F K Brunner (Kensington), B Garfinkel (New Haven), J A Hughes (Washington), I G Kolchinskij (Kiev), A I Nefed'eva (Kazan), K Ramsayer (Stuttgart), J Saastamoinen (Ottawa), C Sugawa (Tokyo), E Tengström (Uppsala), and $\mathrm{H}$ Yasuda (Tokyo), L N Marridis (Thessaloniki).

\section{Study Group on Horizontal Meridian Circles}

Chairman: $E \mathrm{H} \varnothing \mathrm{g}$. Members: $\mathrm{R}$ d'E Atkinson (Bloomington), $\mathrm{J} W$ Gietzen (RGO), G van Herk (Leiden), B L Klock (USNO), J Osorio (Oporto), G J Pinigin and G M Timashkova (Pulkovo), Xu Tong-qi (Shanghai), and Hu Ning-sheng (Nanjing).

\section{Working Group on Astrolabes}

Chairman: G Billaud. Members: S Debarbat, W Gliese, V S Gubanov, F Noel.

A Joint Working Group for coordination of work on the Stellar Reference Frame was established by Commissions 8 and 24 in 1979. The continuation of the WG was approved by Commission 8 , but the question of continuation was not treated by Commission 24 so that, formally, the WG has ceased to exist. Moreover, it seems difficult to have a WG on a subject which is the main subject of the whole commission 8. This difficulty was perhaps the reason that the report given by the Chairman of the WG on 23 August contained considerations on the stellar reference frame rather than a report from the WG.

Should the President of the Commission like to restablish the WG he may of course do so.

\section{SRS Committee}

Chairman: W Fricke. Members: T Lederle, J A Hughes, C Smith, VA Fomin, DD Polozhentsev.

\section{c) Resolutions}

Proposed resolutions on the observation of planets, of high luminosity stars and of HIPPARCOS stars were presented to the Commission and adopted in principle. A committee of $\mathrm{W}$ Fricke, $J A$ Hughes, $E H \varnothing g$ and $Y$ Requieme was given the task of formulation. After a meeting in the afternoon the following resolutions were written and were later endorsed by the IAU.

1. In considering that the determination of the equinox and equator will remain to be an important task of positional astronomy, it is recommended that

(a) the sun and major and minor planets are included in observational programs with transit circles,

(b) the sun and major planets are included in programs with astrolabes, and

(c) minor planets are included in programs of photographic astrometry,

such that from differential and/or absolute observations the 
positions can be rigorously referred to the fundamental

reference system and serve for further improvements of the zero points of this system.

2. In view of the importance of accurate positions and proper motions of High Luminosity stars for galactic research the Commission reaffirms its previous recommendation that such stars be included in transit circle programmes.

3. It is recommended to observe the subset of stars requested by the HIPPARCOS Input Catalogue Consortium. The positions should be available before 1985.5. A moderate accuracy of \pm 1.0 arcsec at that epoch is required, however \pm 0.3 arcsec (in the FK4 system) is desirable.

4. Noting that the HIPPARCOS stars will be selected for their astrophysical and/or astrometric interest and that such stars would traditionally be included in meridian observing programmes, and

noting that very accurate meridian positions of suitable subsets of the HIPPARCOS stars would give checks of the space observations and calibration of the ground-based instruments it is recommended that such subsets, e.g. faint fundamental stars and International Reference stars be observed.

5. A fifth resolution in support of the sydney Observatory was formulated and later passed in agreement with Commissions 24 and 40 .

d) Commission Rules vote.

New rules of the Commission has recently been approved by mail

e) 1980 IAU Theory of Nutation

This theory was adopted by mail vote of the Commission.

f) SRS

A meeting on SRS was held at Brorfelde, Denmark, on 3 March 1982. The full report has been distributed to members of the organizing committee and the conclusions have been accepted for Pulkovo by $K \mathrm{~N}$ Tavastsherna.

The following contains the main points of the meeting:

Participants:

E Høg, President of IAU Commission 8, (Chairman),

$T$ Lederle and $\mathrm{H}$ Schwan, Astronomisches Rechen-Institut (ARI)

$J$ Hughes and C Smith (Secretary), U.S. Naval Observatory (USNO)

The meeting was first proposed by Dr. Tavastsherna in about July 1980 as a meeting on SRS of the president of Commission 8 and representatives from Pulkovo, ARI and USNO. It was therefore now particularly regretted that no representatives from Pulkovo were present, even though the meeting had already been postponed once (from October 1981) in order to make their presence possible. 
It was recommended to the President to appoint $W$ Fricke as Chairman of the SRS Committee. The president has approved this, and it has been accepted by Fricke.

Exchange of Data and Completion of Catalog

It was agreed that ARI would act as the point of exchange of data between USNO and Pulkovo.

Recently USNO has deposited a magnetic tape at ARI of averaged Leoncito RA results of SRS stars referred to FK4 for immediate transmittal to Pulkovo.

A second tape giving Dec in addition to RA was deposited at ARI with the understanding that this second tape would be transmitted to Pulkovo whenever they shall also have deposited a tape of their SRS results with Dec at ARI. A third tape of the Washington six-inch SRS results referred to the FK4 system will be prepared and deposited with ARI for transmittal to Pulkovo under the same conditions.

It is noted that in accordance with the original sRs agreement only averaged results of SRS stars have been exchanged and no results about FK4 stars. A meeting in Heidelberg 1975 recommended the exchange of results from individual nights and of FK4 stars. This is still considered highly desirable. The Perth 70 results of individual nights and of FK4 stars have however been distributed to all parties in 1976.

The meeting agreed to recommend to commission 8 that both Pulkovo and USNO should be free to complete the compilation of the SRS catalog with all data which may be available to them as of the end of 1982 , and that all data whose exchange is now pending wiII have been deposited with ARI by 1 Nov 1982 (i.e. the third USNO tape mentioned above and the Pulkovo Dec).

It must be anticipated that there will regrettably be two different SRS catalogues, one from USNO referred to FK4 and another one from Pulkovo referred to an improved SRS system. The USNO catalog is expected by Hughes to be available by the end of 1983 . The participants in the meeting agreed that the results of the compiled SRS catalogs, once they are completed, will be made available for open distribution to all interested parties.

\section{Meetings on SRS in Patras}

It is recommended to the Chairman of the SRS Committee that he should select members for that committee in correspondence with Pulkovo and the USNO. It is further recommended to the Chairman of the SRS Committee that he schedule a meeting of the committee early in the General Assembly so that reports may be given to Commission 8 before the close of the General Assembly.

Members of the SRS Committee are listed in Ib).
April 1982)

g) Symposium on Astrometric Techniques

The proposed IAU Symposium was supported by the Commission to be held in Venezuela January 1984. Chairman of the Scientific Organizing Comittee: H Eichhorn.

\section{INSTRUMENTATION}

Meeting of Commission 8 ( 19 and 25 August) Chairmen: $E \mathrm{H} \varnothing \mathrm{g}$ on 19 Aug., J A Hughes 25 Aug. On 23 Aug: 
G Billaud: The Photoelectric Astrolabe of CERGA.

The fully automatic instrument employs a full pupil angle of zero expansion material and a Maksutov-Cassegrain reflector. A photoelectric slit micrometer measures the two stellar images, one at a time.

Standard deviations of $0.0038 \mathrm{~s}$ and 0.12 have been obtained for the determination of UTO-UTC and latitude, respectively, from each group of 32 stars.

Y Requieme, J M Mazurier: Bordeaux AMC, Application to Faint Stars. The automatic meridian circle is used for routine differential observations since 1980 and about 18000 observations have been obtained. The tracking photoelectric micrometer with rotating knifeedge sensor is used and mean errors of $0: 11$ and $0: 17$ are obtained in $\mathrm{RA}$ and Dec respectively. With a diaphragm of $20^{\prime \prime}$ diameter a few hundred stars up to $\mathrm{m}_{\mathrm{y}}=12$ were observed and no significant increase of mean error was noticed. The quasar 3C273B fainter than $12 \mathrm{~m}_{5}$ has been observed three times. Average observing time is 70 s per star.

E Høg: Observation of Faint Stars with a slit Micrometer.

A theoretical study has shown that stars of $\mathrm{m}_{\mathrm{y}}=12$ to 13 will be observable in 70 s provided a photomultiplier with low dark current about $5 \mathrm{~Hz}$ is used.

J A Hughes: Progress Report on the Instrumental Development of the Seven-Inch TC.

The development involves the following major components: New objective lens with a solar attachment, a new glass circle, a photoelectric circle scanning system, an automatic telescope setting system, a photoelectric micrometer utilizing an image dissector, and on line computer control.

R Stone: Processing of Images from USNO's Transit Circle Image Dissector.

I V Morrison: Results from the Carlsberg Automatic Transit Circle.

H Yasuda, M Yoshizawa: Tokyo Photoelectric Meridian Circle.

The instrument is being installed this year. It uses a slit micrometer and photon counting techniques. Nadir, zenith mirror, azimuth marks, Sun, Moon and planets will be observable in an automatic mode controlled by on-line computer.

M Miyamoto, Ch Kỉhne: Derivation of Division Corrections from Unbiased Microscope Scales.

B L Klock, T J Rafforty: An Investigation of US Naval Observatory Glass Circles.

Thick transparent glass circles have been used elsewhere, thick enough to carry themselves. The USNO circle is a thin glass annulus cemented onto the conventional steel wheel that was previously directly engraved. High quality division lines and uniform background being stable for many years have been obtained.

E. Høg: Report of Study Group on Horizontal Meridian Circles, by its Chairman.

Since the report of Commission 8 published this year new information on the Pulkovo Horizontal Meridian Circle and the Glass Meridian Circle has been given: 
Declination Observations with the Pulkovo Horizontal Meridian Circle Kirian, Pinigin, Sukharev and Timashkova have (May 1982) given preliminary results of declinations obtained during 3 months:

(1) The photoelectric circle reading gives an mse of $\pm 0: 02$, and the zero point is determined by a pendulum mirror to $\pm 0: 04$ or $0: 06$. A photoelectric ocular micrometer registers the star position in the field of view to $\pm 0: 10$ near zenith.

(2) The ventilation between inner and outer tubes is effective and has reduced the vertical temperature gradient of air inside the tube from a previous $0.5^{\circ} \mathrm{C}$ to $0.1^{\circ} \mathrm{C}$ per tube diameter.

(3) Observations of star declinations show that the equator point does not change with temperature, that the equators obtained from the two tubes coincide and that the final HMC system does not show variations in declination within $\pm 0: 3$. It is therefore concluded that the instrumental flexure of the HMC is small, if at all existing. Further observations will be obtained and the great potential possibilities of this instrument is expected to be realized in the nearest future.

Workshop on the GMC held in China

Dr. Hu Ningsheng arranged lecture and discussion about meridian astrometry and the Glass Meridian Circle with $\mathrm{E} H \varnothing \mathrm{g}$ on 3 and 4 June 1982 at Nanjing. Shanxi Observatory organized a workshop on the GMC at Iintong (near Xian) on 1 to 9 June with 25 participants from Institutes in Lintong, Xian, Beijing, Wuhan, Tianjin, Nanjing and Shanghai, and working papers by $T$ B Andersen, Hu Ningsheng and $E \mathrm{H} \varnothing \mathrm{g}$ on the GMC have appeared in Chinese translation in Scientific Version of Shanxi Observatory B Series, 1982, Vol. 1, pp. 1-79. Høg participated in the Workshop during the last three days, and further cooperation is planned and awaiting approval with Shanxi Observatory, Purple Mountain Observatory, and Nanjing Astronomical Instruments Factory on design study, manufacturing and operation of the GMC.

\section{On 25 August:}

G. Teleki: Report of Study Group on Astronomical Refraction.

A brief review was given on papers written recently that relate to refraction. A summary of the meeting of the working group at Dubrovnik, Yugoslavia. Papers presented there were listed. Particular mention was made of anomalous refraction caused by meridional tilt of atmospheric layers and the possibility of obtaining refractional values directly from astrometric observations.

I S Guseva, E G Zhilinsky, G S Kosin: On the Fifth Edition of the Pulkovo Refraction Tables.

The tables will appear in logarithmic form, as before; however, algorithms in Fortran-4 for the direct computation of values will now appear. These may be used for zenith distances of $0^{\circ}$ to $80^{\circ}$.

I S Guseva: Calculation of the Astronomical Refraction for Different Aerological Data.

The calculations are based on atmospheric physics and optics. An ellipsoidal Earth is used in the computations, which are done by direct numerical integration. This procedure results in a $\Delta \mathrm{R}<0: 05$ at $85^{\circ}$ zenith distance. 
A S Kharin: On the Refraction Anomalies from Meridian Observations. A study of refraction anomalies was presented. A vertical circle has been used to make the necessary measures.

R Fukaya, M Yoshizawa: Numerical Calculation of the Atmospheric Refraction at a Given Wavelength Region.

Numerical calculations based on meteorological data and a spherical, concentric model of the atmosphere. Currently investigating atmospheric tilt. Comparisons with the pulkovo tables show agreement to within $0: 02$ from $0^{0}$ to $75^{\circ}$ zenith distance. The work illustrates that refraction, in its first order theory, can be determined by the meteorological data in the region of the telescope objective.

\section{STELLAR REFERENCE FRAME}

Joint Meeting of Commissions $8+24$ (23 and 25 August) Chairmen: E Høg on 23 Aug., J A Hughes 25 Aug.

On 23 August:

A resolution was proposed by C A Murray requesting the General Assembly to note with concern the decision to terminate the astronomical work at the sydney Observatory and to urge that the sky Survey continues and that the instruments at the observatory remain available for observations. The resolution was endorsed by Commission 40 and passed by a vote of Commissions 8 and 24 .

H K Eichhorn: Report of IAU Commission 8 Working Group on the Stellar Reference Frame.

A review was given of present and possible future needs of the accuracies of various sets of stars that define the stellar reference frame at various magnitude intervals (FK4, GC, $A G$ and $A C$ ). Future extensions of the fundamental catalog, beyond FK5, were discussed and it was recommended that these involve improvement of the positions and motions of the GC stars. The need for an improved SAO catalog was also emphasized.

W Fricke: Report of the SRS Committee.

This report was deferred since C Smith was to report on the compilation of the SRS.

W Fricke: Status of Work on the FK5.

A review was given of the resolutions that began the work and led to the adoption of the 1976 system of astronomical constants. 260 catalogs observed after 1900 contribute 55,000 positions to the new system. There are 30 new absolute catalogs being used for the improvement of the fundamental system. In addition, there are $100 \mathrm{new}$ differential catalogs as well as 130 catalogs, observed 1900 to 1950, that will contribute to the faint fundamental extension. Corrections to the FK4 were shown. The $\Delta \alpha \delta$ exhibit the largest corrections in the zone $-60^{\circ}$ to $-80^{\circ}$ while the $\Delta \delta \delta$ show large oscillations in this region. The current schedule for the completion of the work is: 1. By 1 January 1984 the system will be complete. 2. After January 1984 tables giving the conversions from FK4 to FK5 will be available. 3. By the end of 1985 all of the FK5 will be completed.

C A Murray: Measurement of the Cape Survey, CPC2. progress in measuring the Cape plates on the GALAXY machine was 
reported. 3000 of the 5000 plates, $-40^{\circ}$ to -900 , have been measured. The internal precision was reported as $\pm 0: 1$, and the limiting magnitude is $10 \mathrm{~m} 5$.

C Smith (read by J A Hughes): Progress Report on a Revision of the IRS. The distribution of the International Reference Stars (AGK3R \&

SRS) has been studied to identify regions of low density. It is proposed that about 1200 stars be added to the IRS, mostly in the zone $0^{\circ}$ to $-30^{\circ}$, to give the list a more uniform density.

C Smith (read by $J$ A Hughes): Progress Report on the Compilation of the SRS.

A preliminary SRS catalog has been formed from the results of those observatories that reported both FK4 and SRS observations. The mean errors of the preliminary positions are \pm 0.007 in right ascension and $\pm 0: 12$ in declination. The final SRS will be available by the end of 1983 .

T E Corbin: The Proper Motions of the Southern IRS (SRS) Stars.

A preliminary system of 4968 positions and proper motions on the system of the FK4 has been computed from 18 catalogs that were observed using screens. This system will now be used to study magnitude equations in other southern catalogs and reduce them to the FK4 system.

Y Yatskiv: Review of Astrometric Work in the USSR.

The catalog of Pulkovo observations in the zone $-47^{\circ}$ to $-90^{\circ}$

has been completed. The catalog contains 5511 SRS, 829 BS and

280 FK4 stars. The mean error of a single observation is $\pm 0.014 \mathrm{sec} \delta$ in right ascension and $\pm 0: 39$ in declination. Kiev has begun

compilation of the Bright Star Catalog. The contributing catalogs have been listed and the work of combining them will begin at the end of this year. A list of high-luminosity stars has been proposed for observation. Pulkovo has prepared the list which contains about 2000 stars, mostly in the 6 th to 9 th magnitude range. The Ratan 600 radio telescope has made observations of Mercury and venus. The mean errors of a single observation in right ascension are \pm 0 S 1 sec $\delta$ for Mercury and $\pm 0.06 \mathrm{sec} \delta$ for Venus.

J A Hughes: Progress Report on the Proposed New Zealand Program. The 7" Transit Circle at latitude -4198 in New Zealand and the 6 " Transit Circle at +38.9 in Washington will simultaneously observe the FK5 and IRS (AGK3R \& SRS). The program is to begin in 1983, and the observations of the two instruments will overlap by $60^{\circ}$ and will be made fundamentally.

$Y$ Requieme: Ground-based Measurements for the HIPPARCOS Input Catalog. The Input Catalogue will require epoch 1988.0 positions of $40,000$ bright stars ( $B<10 \mathrm{mag}$.$) with accuracies of \pm 1: 0$ and of 10,000 faint stars $(10<B<13 \mathrm{mag}$.$) with accuracies of \pm 1: 5$. Some 20,000 to 30,000 may need additional observations to obtain the required accuracy. The Cape survey should provide many of the southern positions, and the Bordeaux automatic meridian circle has been proposed to make the necessary observations in the north.

E Høg: The TYCHO Reference System of 400000 Stars.

An exploitation of the HIPPARCOS star mapper has been approved by ESA as the TYCHO project. This project will give positions of at least 400000 stars brighter than $B=11$ with an accuracy at $B=10$ of 
0.03 arcsec. An accurate stellar reference frame with 10 stars per square degree can thereby be established.

Presented by Xu Tong-qi: A Preliminary Report of the General

Catalogue of Stars observed with Photoelectric Astrolabes in China, and: Some Observations of Bright Radio Stars by Means of Astrolabes in China.

The results of eight series of astrolabe observations have been combined to form the General Catalogue (GCPA). This has resulted in mean errors of $\pm 0 \$ 0033$ in right ascension and \pm 0.058 in declination in the GCPA positions. The GCPA is used to investigate errors in the FK4 and comparisons were given in the form of several figures. Of the 1579 GCPA stars, 642 are from the FK4, 516 are from FK4 sup and 421 are from the GC. Work has begun on the problem of the radiooptical connection. Since 197915 radio stars have been observed with the astrolabe at Shanghai (from the list endorsed by Commission 24). Observations of Algol have been given special attention.

H Yasuda, K. Hurukawa: Northern PZT Star Catalog.

Results from ten instruments have been combined to form the NPZT with a mean epoch of 1974. The catalog contains 1719 stars with catalog positions whose average mean errors are \pm 0.0033 sec $\delta$ in right ascension and $\pm 0: 079$ in declination. Comparisons were made with $A G K 3 R$ and AGK 3 to look for magnitude effects, but none were found.

E Marcus: Main Features of the Bucharest NPZT Reference Star Results. A program of 951 NPZT stars was observed using the Bucharest Meridian Circle. Using AGK 3 proper motions the Bucharest results were compared with the Tokyo $\mathrm{NPZT}_{74}$. The computed internal precision is $\pm 0 \$ 016$ in right ascension and \pm 0.29 in declination. The comparison with Tokyo gave external errors of \pm 0 S. 019 in right ascension and $\pm 0: 21$ in declination.

F Laclare, F Chollot: Observations du Soleil avec Astrolabe. Astrolabes at Sao Paulo and CERGA began observing the Sun in 1974 and 1975, respectively. They are now involved in a joint effort to regularly observe the sun. Utilizing prisms that allow observations at zenith distances of $30^{\circ}, 45^{\circ}$ and $60^{\circ}$ each can cover 708 of the orbit.

G Westerhout: Concluding Remarks. (These had not been received at the time of writing).

On 25 August:

S N Sadzakov, M D Dacic, V A Fomin: An Investigation of the Systematic Errors of the Belgrade NPZT and AGK3 Catalogues on the Basis of Modern PZT Observations.

The NPZT catalog was observed at Belgrade from 1972 to 1980. The accuracy was reported to be $\pm 0 \$ 012 \mathrm{sec} \delta$ in right ascension and $\pm 0: 10$ in declination. Comparisons were made for several different stations.

S Sadzakov, V A Fomin, M Dachich, D Saletich: Systematic Errors in Latitude Observations of Various observatories.

Work by Yasuda and the AGK 3 were used to estimate the external accuracy of the NPZT. Curves of $\Delta \alpha_{\alpha}$ and $\Delta \delta_{\alpha}$ were shown. The systematic component was shown to be due to the systematic errors in the AGK3 proper motions. 
J Phan Van: Observations Planetaires a l'Astrolabe du CERGA.

The results of observations of Mars and Jupiter made in February and March of 1980 at CERGA. A $45^{\circ}$ prism was used. Comparisons were made with Washington 6" observations for the same period that show systematic differences between the two series of observations. The differences are in the 1" to 2" range in both coordinates. However, subsequent discussions revealed that the two sets of observations had been treated differently with respect to $\Delta T$.

G Vigouroux: Catalogues Astrolabes a 2 Distances Zenithales.

G Carrasco, P Loyola: RA Observations with the MC of Cerro Calan 1978 to 1981.

The Repsold Meridian Circle at Cerro Calan started observing the FKSZ stars in December 1979. In addition, FK4 stars have been observed over a wide arc, from $+42^{\circ}$ to -900 and from $-90^{\circ}$ to $-68^{\circ}$ lower culmination. Comparisons of Instrument - FK4 in right ascension with similar differences from other observatoris show very good agreement.

\section{EXTRAGALACTIC REFERENCE FRAME}

Joint Meeting of Commissions $7,8,24,33,40$ and 44 (21 August)

Chairman: A N Argue

An extensive report will be given elsewhere of papers by: Argue; Johnston; Eichhorn; Kovalevsky and Froeschle; Lindegren; Chollet and Debarbat; Fanselow, Purcell, Sovers and Thomas; Hemenway; de Vegti Quijano. 
Report of Meetings: 18,20,21,24 and 25th August 1982

PRESIDENT: E.H. Richardson

\section{Report of President}

Since the 17th General Assembly in Montreal, the major activity organized by Commission 9 was IAU Colloquium 67, held 8-10 September, 1981, on the observing floor of the 6 metre telescope of the Special Astrophysical Observatory, USSR. The Proceedings have been published by D. Reidel Company, entitled "Instrumentation for Astronomy with Large Optical Telescopes". Commission 9 also participated in 'ICO-12', the Congress and Twelfth Assembly of the International Commission for Optics held at Graz, Austria, 31 August to 5 September 1981. The papers presented at that meeting have been published in optica Acta, 1982, vol. 29.

The scientific and technical reports of the Commission 9 meetings at the 1982 General Assembly are arranged below according to the Working Group indicated, with the exception of the general meeting on 20th August which combined the interests of the Working Group on Large Telescopes and the Working Group on Infrared and General Techniques.

Business Meeting, 21st August 1982

In anticipation of a poor attendance at the meeting scheduled for 21 st August (which proved to be so), the election of new officers was started at the end of the well-attended general meeting of 20th August and the results were confirmed at the meeting on 21 st August.

Dr W.C. Livingston was elected President, there being no other nominations. For the office of Vice-President, two nominations were received and a ballot was held. Nominations for membership of the Organising Committee were made by the outgoing President and Vice-President in consultation with the Commission members who were present. This resulted in the election of the following:Vice-President: C.M. Humphries; Committee members: J.C. Bhattacharyya, J. Davis, J.L. Heudier, I.M. Kopylov, G. Lelièvre, J.P. Maillard, A.B. Meinel, N.N. Mikhel'son, E.H. Richardson (Retiring President), J. Ring, J. Rösch, N. Steshenko, R.G. Tul1, M.F. Walker, W.L. Wilcock, G. Wlérick.

Dr Richardson reported that in early 1982 all members of Commission 9 who could be reached by mail were asked to reply if they wished to remain members. Those who did not reply were removed from the membership list unless another member spoke on their behalf. It was agreed that the names of all new applicants should be added to the membership list.

General Meeting, 20th August: TELESCOPES AND INSTRUMENTS (Chairman: E.H. Richardson)

The following communications were presented: 
LEST - A SUGGESTED DESIGN FOR A NEXT GENERATION SOLAR TELESCOPE (A. Wyller; Stockholm Observatory)

Design of the proposed Large European Solar Telescope (LEST), initiated by the Joint Organisation for Solar Observations, is proceeding on the basis of a specification for an instrument of large aperture combined with high spatial resolution (< $0.3 \mathrm{arcsec}$, preferably $0.1 \mathrm{arcsec})$, tracking at this accuracy for at least $30 \mathrm{~min}$, low intrinsic polarization properties, and an adequate field of view (2 arcmin minimum, 30 arcmin preferably). The Ritchey-Chretien optical design is based upon that of the NASA Space Telescope with a $2.4 \mathrm{~m}$ honeycomb primary (f/2.3) and a $0.84 \mathrm{~m}$ diameter secondary. To minimise thermal problems, evacuated and He filled tube structures have been considered (see next paper). The telescope will be built on a tower of minimum height $30 \mathrm{~m}$ with a cap dome similar to that used by Rösch at Pic-du-Midi, and is likely to be located either at La Palma or at Tenerife.

TESTS OF A HELIUM FILLED SOLAR TELESCOPE (R.B. Dunn, O. Engvold, W. Livingstone and R. Smartt; Kitt Peak National Observatory). With additional presentation by J. Rösch, Pic-du-Midi Observatory.

Since helium has $1 / 8$ th of the refractive index (in terms of $n-1$ ) of air and almost 10 times greater heat conductivity, it should be capable of reducing thermal problems in a sealed telescope tube structure without the need for evacuation. In order to test this, the internal seeing of the KPNO 'vacuum' telescope was evaluated from video recorded interferograms obtained with the solar image present, at various pressures of air and of helium. At evacuated air pressures up to 25 Torr, motion of the fringe pattern could just be detected whereas at higher pressures the fringes became more and more chaotic until, at atmospheric pressure, the fringes were barely visible. When the tube was filled with helium, no image motion occurred until the pressure had increased to 650 Torr. The conclusion is that a helium filled solar telescope at normal atmospheric pressure will hardly differ from an evacuated one. Furthermore, the helium removes heat effectively from the optical surfaces, reducing thermal effects such as distortion and change of focus.

MICRO-ARCSECOND ASTROMETRIC ORBITER CONCEPT (S.M. Rathjen, D.C. Black, J.P. Murphy, L.W. Bandermann and W.H. Alff; NASA Ames Research Center and Lockheed Palo Al to Research Laboratories)

A shuttle launched free-flying satellite characterised by the ability to make astrometric measurements with exceptionally high angular resolution is being proposed. The mission objective is the discovery and characterisation of other planetary systems which in turn would lead to a better understanding of the formation and evolution of our own solar system. The instrumentation consists of a telescope which feeds an optical system utilising a Ronchi grating, an apodized pupil and a split-field detector giving an angular resolution capability of $10^{-5}-10^{-6}$ arcsec. With a $1 \mathrm{~m}$ diameter telescope aperture and 1 hour integrations, such an instrument would allow dozens of stars to be searched for Earth-mass planets and thousands of stars for Jupiter-mass planets. The concept is based on a 5 year mission, refurbishab1e, with 10 acquisitions per year per target star.

\section{ULTRA-THIN MIRRORS (E.J. Hysom; A.E. Optics Ltd., Luton, England)}

For some time now, A.E. Optics has been making mirrors of diameter $23 \mathrm{~cm}$ and thickness $1 \mathrm{~cm}$ which were figured to a moderate astronomical quality. Recently we received a request for mirror substrates of diameter $80 \mathrm{~cm}$ and thickness $3 \mathrm{~mm}$ $(d / t=270 / 1)$ with a focal ratio $\sim f / 6 ; 81$ such mirrors were required by the Rutherford Appleton Laboratory for use in a tessellated mirror Cerenkov detector. The method of manufacture consisted of preforming to the required radius by slumping the substrates onto a steel mould. At the early testing stage, difficulties were encountered with an edge effect due to the extreme thinness. To 
ease manufacturing problems it was agreed to increase the thickness to $4 \mathrm{~mm}$, and work the substrates at $80 \mathrm{~cm}$ diameter before edge cutting to hexagons of circumscribed diameter $70 \mathrm{~cm}$. It is considered that increasing the thickness still further to $\sim 10-12 \mathrm{~mm}$ would permit figuring to give an image size which would be acceptable for certain types of astronomy, whilst still retaining the cost advantage that results from their low weight. It is estimated that a $1 \mathrm{~cm}$ thick $70 \mathrm{~cm}$ diameter substrate could be worked relatively quickly to give an image size of a small fraction of a millimetre and a number of such mirrors driven by stepping motors could form the basis of a cheap multi-aperture telescope.

ACTIVE OPTICS INSTRUMENT FOR USE AT MAUNA KEA (Laird A. Thompson; Univ. of Hawaii).

An active optics image stabilising instrument is currently under construction for use at Mauna Kea Observatory. It is designed to remove image jitter with frequencies $<70 \mathrm{~Hz}$ and with amplitudes $<13$ arcsec. There is a special need for such an instrument at Mauna Kea because stellar images, when viewed from this site, shrink to diameters $<0.5$ arcsec. When this happens the image core almost invariably shows a residual motion with a frequency $\sim 20 \mathrm{~Hz}$ and an amplitude $\sim 0.5$ arcsec. Until such image jitter is removed, full advantage cannot be made of the superb Mauna Kea conditions.

The primary optical element in the image stabilizing system is an active mirror which folds the incoming converging Cassegrain beam by $45^{\circ} \pm \Delta \theta$, where a range of $\Delta \theta<16$ arcmin provides image mobility of \pm 13 arcsec in the image plane. The active mirror, $12.5 \mathrm{~cm}$ in diameter, gives an unvignetted field of view of 8 arcmin $\times 11$ arcmin. The mirror is controlled by four linear motors (Ling drivers) which are linked in pairs to provide two axes of motion. Image position can thereby be controlled to within \pm 0.01 arcsec in the image plane. Following the active mirror the converging beam intercepts a beam splitter (or alternatively a small central mirror) which provides two optical paths: one to the Cassegrain focal plane and the second to the guide probe. Freezing image motion at the guide probe (by feedback to the active mirror) simultaneously freezes image motion at the instrument focal plane.

As a guide probe, we use a set of 5 closely packed optical fibres linked individually to 5 photoelectric cells. The guide probe gives not only an error signal for controlling image motion, but also provides a rea1-time measure of the image quality. The image quality information is used to control an active shutter placed immediately above the Cassegrain focal plane. When the image quality deteriorates momentarily, the active shutter closes to maintain excellent image quality.

PRIME FOCUS CORRECTOR LENS FOR A 300-INCH (7.6M) TELESCOPE (E.H. Richardson, C.F.W. Harmer and W.A. Grundmann; Dominion Astrophysical Observatory)

A new design of three-element prime focus corrector lenses for RitcheyChrétien ( $R-C)$ telescopes has superior resolution and a greater back focal distance than previous designs. In the older design where the size is restricted, the resolution would decrease if the lenses were enlarged.

The proposed 300-inch Texas telescope made severe demands on the design of a corrector: the primary focus is very fast, $F / 2$, and a resolution of 0.5 arcsec was sought over a very wide spectral region. It has been found from this work that exceptionally fast Ritchey-Chretien telescopes can now be well corrected at the prime focus with three-element corrector lenses covering a wide field and a broad spectral region. Although larger than previous refractive lens designs, they are feasible and still have much smaller central obstruction than corrector mirrors. 
UKIRT INSTRUMENTATION (W.J. Zealey; Royal Observatory, Edinburgh)

Improvements in the mechanical performance of the United Kingdom Infrared Telescope have continued with the installation of new RA and Dec position encoders (giving 3 arcsec RMS pointing accuracy), the provision of secondary mirror chopping (max. amplitude \pm 100 arcsec at $10 \mathrm{~Hz}$, the re-writing of all control software in Fortran (instead of the less flexible assembly language), and an improvement in the magnitude 1 imit $(V=18.5)$ for which autoguiding can be used. Fully commissioned instrumentation comprises InSb photometers for use with $\mathrm{J}, \mathrm{H}$ and $\mathrm{K}$ filters or $\mathrm{CVF}(\mathrm{R}=130,1.4$ to $2.8 \mu \mathrm{m})$; InSb photometers for use at $J, H, K, L$ and $M$ (optimised at $L$ and $M$ ) or with CVF ( $=100,2.3$ to $4.6 \mu \mathrm{m})$; a Ge bolometer ( $L$ to $35 \mu \mathrm{m}$ ); and a cooled grating spectrometer (single channel, 1 to $5 \mu \mathrm{m}, \mathrm{R}=500$ at $2.0 \mu \mathrm{m}$ in first order). Instrumentation under construction includes a 7 channel InSb array spectrometer with option of a 2 to $5.3 \mu \mathrm{m}$ Fabry-Perot system; an 8 channel bolometer with 4 spatial channels at any two of 10,20 or $34 \mu \mathrm{m}$; a 16 channel SiAs array photometer $(10,20 \mu \mathrm{m})$; and a

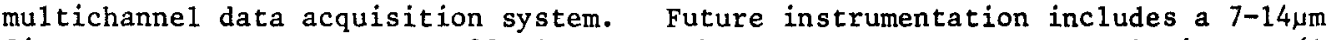
SiAs array spectrometer; a 32 element InSb array spectrometer, a polarimeter (1 to $5 \mu \mathrm{m})$; a ${ }^{3} \mathrm{He}$ cooled submillimetre photometer ( $350 \mu \mathrm{m}$ to $1 \mathrm{~mm}$ ); and heterodyne receivers for use at submillimetre and millimetre wavelengths. At the Cassegrain focus ( $f / 9$ or $f / 35$ ), up to six instruments can be mounted simultaneously. Effort has also been directed towards achieving remote operation of the telescope from the sea-level base at Hilo and from the Royal observatory, Edinburgh (note added after the meeting: the first successful link giving remote control and direct data transfer between UKIRT and Edinburgh using the TELENET satellite and Packet Switching Service occurred on 6 September 1982).

\section{CFHT INFRARED INSTRUMENTATION (J.P. Maillard)}

Infrared instrumentation at the Canada-France-Hawaii Telescope has been tested and commissioned recently (October 1981 to April 1982). The telescope is now equipped with an infrared top end holding a chopping $f / 35.5$ secondary mirror (maximum throw 6 arcmin, frequency 1 to $50 \mathrm{~Hz}$ ). Each instrument using this focus is mounted on a Cassegrain adaptor providing acquisition of the field and guiding. Two photometers ( $\mathrm{InSb}$ and $\mathrm{Ge}$ bolometer) provide complete coverage from 1.25 to $33 \mu \mathrm{m}$ by discrete filters and CVFs. A high resolution 1-5 $\mu \mathrm{m}$ interferometer ( $60 \mathrm{~cm}$ path difference) is also part of the Cassegrain instrumentation and is completely computer controlled. Preliminary photometric tests at the telescope show that a magnitude 1 imit of 19.3 at waveband $K$ can be reached in 1 hour with $\mathrm{S} / \mathrm{N}=1$. First results with CVF spectra in the 3-5um region of some young objects provide evidence that their envelopes are composed of different grains of silicate.

FOURIER TRANSFORM SPECTROMETER FOR THE IRTF ON MAUNA KEA (Reinhard Beer; Jet Propulsion Laboratory, Pasadena)

This is a dual input, dual output, step-and-lock spectrometef for the region 1.1 to $5.5 \mu \mathrm{m}$, extendable to $20 \mu \mathrm{m}$. Resolution is $0.01 \mathrm{~cm}^{-1}$ with a maximum scan rate of 400 steps/sec and a path difference accuracy $<0.5 \AA$ peak to peak in $1 \mathrm{~Hz}$ bandwidth (< $10 \AA$ in $3 \mathrm{KHz}$ bandwidth). The reflective optics are sapphire overcoated silver mirrors giving an instrument transmittance of $>75 \%$ (beam size $25 \mathrm{~mm}$ ), the beam splitters are either $\mathrm{Si}$ on $\mathrm{SiO}_{2}$ or $\mathrm{Si}$ on $\mathrm{CaF}_{2}$, and the detectors are InSb housed in I.R.L. dewars with overcoated $\mathrm{ZnSe}$ relay and field lenses. The instrument is equipped with a wide range of filters and has built-in facilities for laser alignment and test-lamp calibration. The data handling system utilises two lock-in amplifiers with programmable gain control, digitised to 12 bits, a 24 bit integrator/ratiometer, and a DEC PDP/8E contro1 computer with $66 \mathrm{M}$ byte disk for interferogram co-adding, and tape facilities for off-line interferogram transformation. Individuals with appropriate observing facilities who might be interested in using this instrument for their own programs are urged to contact the author. 
RESULTS WITH THE HYDROGEN FLUORIDE PRECISION RADIAL VELOCITY TECHNIQUE

(B. Campbe11; CFHT Corpn., and A. Irwin; Univ, of Victoria)

This method utilises a hydrogen fluoride absorption cell to superimpose reference wavelength 1 ines upon coude spectra. A series of high $\mathrm{S} / \mathrm{N}$ spectra of Arcturus obtained on two nights gave radial velocities with internal errors of $6 \mathrm{~m} \mathrm{~s}^{-1}$. There is, however, a change in velocity of about $100 \mathrm{~m} \mathrm{~s}^{-1}$ between the two nights. This is likely to be real since similar series of spectra of Procyon on the same two nights show no velocity variations. Additional observations of Procyon on nine other nights suggest that any systematic errors in this velocity technique are less than $20 \mathrm{~m} \mathrm{~s}^{-1}$.

\section{THE NEW DIFFERENTIAL PHOTOMETRY SYSTEM OF THE UNIVERSITY OF CALGARY}

(E.F. Milone)

The Rapid Alternate Detection System developed at the Rothney Astrophysical Observatory over the past decade for differential variable star photometry uses a single telescope, photometer and detector, and consists of gated, pulsecounting electronics synchronized to a chopping secondary. The mirror is successively driven (Ling driver, \pm 10 arcmin maximum throw) to four positions permitting rapid alternate detection of a variable star, comparison star and two sky background regions. The system compensates adequately for the effects of transparency, sky brightness, and detector sensitivity variations occurring on timescales longer than the system's duty cycle, and has been used successfully with as much as three magnitudes of cloud cover and attendant sky brightness variations. Information about this system is also being communicated to Commission 25.

\section{SOME RECENT CHINESE INNOVATIONS IN INSTRUMENT DESIGN (T. Kiang; Dunsink Obser-} vatory)

A "photoelectric astrolabe" of novel design was completed in China in 1974. It differs from the Danjon Astrolabe in the following respects: (1) its optical components are placed in an evacuated chamber and the light enters through two horizontal windows on the upper side. In this way, all refraction effects that can be attributed to a plane-parallel atmosphere are eliminated. The vacuum also keeps the mercury horizon clean for a very long time; (2) the Danjon $60^{\circ}-$ prism is replaced by a side-by-side split mirror receiving the direct and reflected stellar beams; (3) reflecting surfaces are used exclusively. Thus, there are no chromatic effects and larger apertures are possible (actual aperture $=20 \mathrm{~cm}$ ); (4) a Wollaston prism is not required. The direct and reflected images traverse a reflecting-transmitting grid and the phase of the cut-off signals produced by the grid is registered photoelectrically. In this way, all the light from a star is utilized. A detailed description in English can be found in the translation journal Chinese Astronomy 1977, 1, pp.79-87. The following are some test results, compared with results from parallel observations using a Danjon instrument (in brackets): precision of a single star position: \pm 0.14 ( \pm 0.21$)$; closing error in the instrumental almucantar: $0.13(+1.67)$; therma1 correction $-0.1025 /{ }^{\circ} \mathrm{C}\left(+0.160 /{ }^{\circ} \mathrm{C}\right)$. Routine observations with this Chinese photoelectric astrolabe have been in operation in three observatories (Shanghai, Beijing and Yunnan) and the results obtained are generally accessible in Chin. Astron.

Beginning with Vol. 5 (1981), this translation journal was re-titled Chinese Astronomy and Astrophysics. A recent issue (C.A.A. 6, No. 2, 1982) contains reports on two minor innovations. One refers to a three-channel photometer capable of uninterrupted monitoring of a program star, a comparison star and a control star. Three photoelectric multipliers are used and are mounted on three of the four arms of a cross, rotatable in its own plane. The other concerns the chopping arrangement in an IR detector. Unlike the design in general use, the rotating chopper is used to feed in the sky background only, 
while the fixed mirror feeds in star + sky. This simple exchange of roles reduces excessive demand on the setting and alignment of the chopper.

A SCANNING MICROPHOTOMETER FOR ON-IINE DATA REDUCTION (H. Lorenz; Zentra1institut für Astrophysik, Potsdam)

The measurement of astronomical plates using a scanning microphotometer gives information of high redundancy together with considerable noise. On the basis of HAAR-transform a processing is described which separates the noise optimally by a further variable digital filter (KALMAN filter). The system fits its own bandwidth to the incoming signal and reduces the number of data by a factor of up to 100 . For the measurement of astronomical photograms, an evaluation system (ASAG) is planned in cooperation with VEB Carl-Zeiss-Jena. The system will include software packages for different applications and ensure high mechanical accuracy and stability (air bearings, laser metering). The method used allows an improvement in sensitivity for the evaluation of faint objects in the background by $1-3 \mathrm{mag}$.

DESIGN FOR A U.K. 18M TELESCOPE (A. Boksenberg; Royal Greenwich Observatory) Design studies carried out at the RGO for an optical/IR telescope of effective aperture $\sim 18 \mathrm{~m}$ favour a multi-mirror concept. The configuration being studied comprises six close-packed $\mathrm{f} / 2$ paraboloids (diameter $7.5 \mathrm{~m}$ ) which form an integrated, filled aperture in a cell supported by two sets of relatively short but stiff trusses of an altazimuth mounting; each element of the aperture is a two-mirror system equipped with $0.5^{\circ}$ field corrector. The particular advantages of this arrangement are: (1) on-axis optics can be produced more easily than off-axis; (2) the use of two-mirror systems removes the requirement for excessively strict tolerances on focal lengths; (3) each component of the aperture is optically clean and suitable for IR work; (4) it can be used as an aligned, coherent array; (5) flexibility for the efficient matching of instrumentation to the telescope beam by using either multiple instruments at the individual foci or, for large instruments, the combined focal station.

THE NTT AND VLT PROJECTS OF ESO (J.P. Swings; Institut d'Astrophysique, University of Liege)

The New Technology Telescope (NTT) being built by ESO for completion in $1986 / 87$ is intended as a test for several new design concepts before embarking on the much larger VLT project. These include: weight reduction; unique type of focus; compact rotating building; closed loop active control of thin primary mirror (aspect ratio 15/1); reduced auxiliary instrumentation; remote control. With an aperture of $3.5 \mathrm{~m}$, its approved budget is DM 24 million (1982) which is less than a third of that for the existing $3.6 \mathrm{~m}$ telescope at La Silla.

The VLT (Very Large Telescope) is conceived as a powerful new instrument of large aperture with a capability for interferometry. The proposed design consists of four separate telescopes, each with aperture in the range $8-10 \mathrm{~m}$, arranged as an array in which the beams can be combined by coherent or incoherent addition. With an equivalent aperture of $16 \mathrm{~m}$ and diffraction 1 imited imaging in the infrared, the VLT is likely to be used particularly for high resolution spectroscopy of faint objects and interferometry at optical and IR wavelengths.

Working Group on Detectors (Chairman: W.C. Livingston)

At a meeting of the Working Group held on 24 th August the following papers were presented:- 


\section{A) Electronography}

PROGRESS OF THE ELECTRONIC CAMERAS AND PHOTOCATHODES PRODUCED AT PARIS OBSERVATORY (G. Wlérick, M. Duchesne, B. Servan, F. Gex and J.M. Munier)

Production and research: The photocathode laboratory, which has been transferred to a new building and includes clean room facilities, produces about 200 photocathodes each year: S11 cathodes (20 to $81 \mathrm{~mm}$ ), S20 cathodes (20 to $30 \mathrm{~mm})$. Recent developments include $S 20$ cathodes of $81 \mathrm{~mm}$ diameter and $\mathrm{S} 1$ infrared photocathodes. Two cameras for the C.F.H. telescope have been described at the Zelenchuk meeting (IAU Colloquium 67):- (1) the 40mm camera with electrostatic focusing, developed by M. Duchesne, (2) the $81 \mathrm{~mm}$ "camera electronique grand champ" which, since the retirement of A. Lallemand, has been operated by $B$. Servan and G. Wlerick; these cameras are due to be shipped to Hawaii before March 1983.

Observations at Haute Provence: (i) Infrared: In January 1982, M. Duchesne and $Y$. Andrillat made observations with a camera equipped with an S1 cathode that was both sensitive and homogeneous. They took plates of emission line

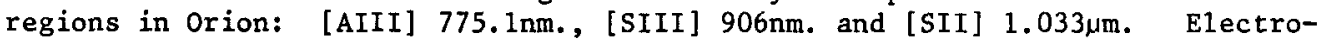
nographic cameras are probably the best receivers for monochromatic studies in the 1 to $1.1 \mu \mathrm{m}$ region since they are free from interference fringes; (ii) Camera grand champ: This camera has been operated mostly by G. Wlérick, B. Servan, V. Cayatte and L. Renard. Different programmes have been carried out: studies of radio sources including the separation of the components of BL Lac type objects (in collaboration with $\mathrm{A}$. Bijaoui); stellar photometry (open cluster M37, studied by A. Robin); Saturn's rings (Sicardy et al.), gravitational lenses $(0957+561$, Vanderriest et al.).

Studies of electronographic emulsions: To choose the most convenient emulsion for a particular observation, G. Wlérick, V. Cayatte, A. Sellier and D. Michet have been engaged in a systematic photometric programme to determine parameters such as characteristic curve, speed, signal/noise ratio, and homogeneity. Whereas the emulsions show very different speeds, the signal/noise ratio is not very sensitive to the emulsion type and to the choice of developer.

\section{ELECTRONOGRAPHIE AVEC LE TELESCOPE CFH (G. Lelievre)}

$D^{\prime}$ ici un an, les trois types de cameras electroniques seront operationnelles au T.C.F.H.: (1) La caméra électrostatique de $40 \mathrm{~mm}$ dévelopé par $M$. Duchesne est destiné a la spectroscopie avec le spectrographe a fente longue ainsi qu'avec le spectrographe multifentes. M. Duchesne a dû résoudre des problemes difficiles sur les flexions et l'influence du champ magnétique terrestre afin de respecter les tolérances imposees: les images ne doivent pas bouger de plus de $4 \mu \mathrm{m}$ pour des poses superieures a $1 \mathrm{H} \mathrm{a} 45^{\circ}$; (2) La caméra magnétique "grand champ" est décrite par G. Wlérick; (3) La camera électrostatique a vanne a été presenté a Zelenchuk en septembre 1981. E1le est régulièrement utilisee au foyer primaire du TCFH. Les résultats les plus marquants concernent les mesures UBV de la galaxie responsable du quasar double $0957+561$ ainsi que les sondages profonds pour donner une 1 imite de la magnitude limite du noyau de la comete Halley: $m_{v}>25.8$ à 3 sigmas.

Pour faciliter les conditions d'observation, le support d'instrumentation au foyer primaire a éte reconstruit af in de permettre d'empiler les instruments suivants:- correcteur grand champ; réseaux objectif; bonnette; caméra électronique. Avec des images de 0.7", les magnitudes limites attendues sont: $B=26.5$ en photometrie et $V=23$ pour la spectroscopie des raies d'emission des quasars. Une nouvelle camera, avec une photocathode de diametre $40 \mathrm{~mm}$ est en cours de réalisation pour remplacer le modele actuel de $30 \mathrm{~mm}$. 
ELECTROGRAPHIC CAMERAS AT BEIJING OBSERVATORY (Fu Delian)

A Kron camera which has been developed has been tested and proven to be reliable. The working field is only $12 \mathrm{~mm}$ in diameter owing to simplified electron optics. Details of a novel gate valve are given. Cs-Sb photocathodes having a sensitivity of $40 \mu \mathrm{a} / \mathrm{lum}$ are achieved. This camera is presently being evaluated at the telescope. An improved version having better electron optics and hence a larger field $(\sim 40 \mathrm{~mm})$ is being designed. Finally, a magnetic focus camera similar to that built at Meudon is under development. A format of $100 \mathrm{~mm}$ is the goal.

UBV PHOTOMETRY OF STARS IN M3 (H.D. Ables, C.C. Dahn and A.V. Hewitt; US Naval Observatory, Flagstaff Station)

As part of a continuing program to establish UBV sequences for use with small format panoramic detectors, we have measured photoelectrically and/or electrographically 130 stars in an 8 arc min diameter region centered approximately 13 arcmin east of the nucleus of M3. The stars range in brightness from $\mathrm{V}=12.85$ to $\mathrm{V}=21.37$ and almost all appear to be members of $\mathrm{M} 3$. The details of the photometry are discussed and the resulting color-magnitude and colorcolor diagrams are presented.

STELLAR PHOTOMETRY WITH THE $1.5 \mathrm{~m}$ DANISH TELESCOPE AND $90 \mathrm{~mm}$ McMULLAN ELECTRONOGRAPHIC CAMERA (A. Blecha, M. Walker and J. Andersen)

A comparison of B-V photometric results for the HODGE-11 LMC cluster has been made from independent analyses of the same data (two $B$ and two $V 3$ hour exposures), using (1) a Joyce-Loebl microdensitometer at Lick and (2) the Geneva microdensitometer and HP 1000 computer system. Much better plate-to-plate agreement and a more comprehensible colour-magnitude diagram are achieved with the Geneva system working with the sophisticated multiple profile fitting sof tware. In the magnitude range 16-22, a precision of \pm 0.04 RMS is obtained. This program is part of a larger photometric study of LMC and SMC globular clusters.

\section{ELECTROGRAPHY AT PIC-DU-MIDI OBSERVATORY (J.L. Vida1)}

A short review is given of the main results obtained in recent years at Pic-du-Midi using Lallemand-Duchesne cameras. These include (1) studies of Saturn (discovery of the new satellite, Dione B; measurement of the thickness of Saturn's rings and their scattering properties; detection of E-ring), (2) galactic globular clusters and HII regions (map of the core of MI5; discovery of a new breed of nebular condensations in the vicinity of the Trapezium in Orion), (3) extragalactic studies (ionised gaseous content of NGC 1068, supernova in NGC 2903 and in NGC 1199; high resolution plates of the jet in M87).

\section{B) Solid State Detectors}

A WINDOWLESS INTENSIFIED CCD IMAGE SENSOR FOR A FAR-UV ECHELLE SPECTROGRAPH ON A SOUNDING ROCKET (E.B. Jenkins; Princeton University Observatory)

A photon-counting camera to record high resolution spectra $\left(\lambda / \Delta \lambda=2 \times 10^{5}\right)$ of bright stars has been developed at Princeton. Since the spectral range to be recorded spans the Lyman 1 imit and $1100 \AA$, the image must be recorded with a windowless detector. The camera design for this mission uses an opaque photocathode and has an image section with an $18 \mathrm{kV}$ potential difference to accelerate the photoelectrons towards a thinned, back-illuminated RCA CCD ( 320 x 256 active pixels, each $30 \mu \mathrm{m}$ square). A 150 gauss magnetic field directs the electrons at a small angle from the incoming optical beam and focuses them onto the CCD. Each high-energy photoelectron generates several thousand charges, with a lateral spread of about one pixel, within the CCD. 
With the CCD running at 15 frames per second, we obtain a readout noise of approximately 200 electrons rms per pixel, which is about a factor of ten below the charge of a single photoevent. Rapid readout permits us (1) to count relatively high photon fluxes, (2) to run the CCD uncooled, and (3) to bypass the requirement for fine image stablization by allowing frame-by-frame postflight corrections of small translations of the spectrum. In recognising individual photoevents in the data reduction, we can identify the much larger pulses produced by ions and reject them. This is crucial 1 for sounding rocket applications where the vacuum conditions are poor ( $\mathrm{P}>10^{-5}$ Torr). Laboratory tests of the resolution of the image section demonstrate that the electron focus is good over the whole field of view, confirming an earlier calculation of electron trajectories for the oblique magnetic focusing.

The flight test of this detector is expected to occur in November 1982 when the spectrograph will be flown for the first time.

IMPROVED INTERFEROMETRIC TESTING FOR TELESCOPE MIRRORS (M.J. Smyth; Dept of Astronomy, University of Edinburgh)

The production of large precise optical components is a cyclical process of measurement and optical working. A new interferometric measurement system, developed at the University of Edinburgh in collaboration with NEI Grubb Parsons Limited, replaces the conventional photographic method and improves the process by shortening the cycle time and improving accuracy and spatial resolution. It comprises a Reticon silicon diode array detector attached to a wavefront shearing interferometer and microcomputer.

The system images the fringe pattern on the detector array which senses the intensities at 512 points across the mirror diameter. These are stored and the measurements are repeated for a total of 5 stepped phase values. Within 10-15 minutes of measurement and computing time, the microcomputer displays a plot of relative phase across the mirror diameter. Interactive graphics allow $360^{\circ}$ phase discontinuities to be removed, and the display can be folded to allow comparison and superposition of points equidistant from a selected centre of symmetry. For routine measurements of uncoated mirror surfaces, the relative phase precision is $\sim 0.01$ wavelength. The phase data are stored on a floppy disc and converted into wavef ront errors using a summation procedure, giving a precision for the measured wavefront errors of $\sim 0.03$ wavelength (depending on the amount of shear used). For a shear value of $100 \mathrm{~mm}$, the phase precision is equivalent to an image resolution of 0.01 arcsec.

\section{SOLID STATE DETECTOR DEVELOPMENTS AT THE ROYAL GREENWICH OBSERVATORY (P.R. Jorden)}

The RGO is concentrating on developing CCD cameras to be used on the telescopes of the new La Palma Observatory. In 1981 a prototype camera was built for the Anglo-Australian Telescope and a description of this and some performance characteristics of the RCA 53612 CCD can be found in Proc. SPIE 331, Tucson 1982 (Jorden, Thorne and Van Breda). A camera for the 2.5m (INT) prime focus is complete and further cameras for the $2.5 \mathrm{~m}$ Cassegrain and the $1 \mathrm{~m}$ telescope are under construction. A revised multi-detector system is being designed for the $4.2 \mathrm{~m}$ telescope along with a CCD autoguider which will be designed in 1983/84. Problems due to Fabry-Perot interference fringe effects in the RCA thinned $C C D$ are being investigated. We have shown that the patterns generated by night-sky emission lines can be effectively removed.

In the U.K., the General Electric Company are making a "speculative" development of a large CCD for the AAT. RGO, together with the Institute of Astronomy, Cambridge, will be testing this device. The format is $1501 \times 1501$ pixels of size 26 microns square and the noise is expected to be 10 electrons rms. Production is due at the end of this year and we plan to test them early 
in 1983. At present at RGO we are examining the GEC P8600 CCD in comparison with the RCA 53612 device. Other detector applications, including a microchannel plate intensified system, are also being studied.

KAVALUR FAINT IMAGE DETECTION DEVICE (J.C. Bhattacharyya; Indian Institute of Astrophysics, Bangalore)

A system for producing digitally recorded faint spectra at the coude spectrograph focus of the $1 \mathrm{~m}$ telescope at Kavalur is described. It consists of a 4-stage image intensifier coupled to a CCD detection device by a relay lens arrangement. An Intel 8086 based microprocessor generates the stream of pulses for CCD operation and controls the digitization and storage of the data in an auxiliary dynamic RAM. The system permits reading of selected pixels and can handle a variety of two dimensional detector matrices.

SOLID STATE AREA SCANNER PHOTOMETER (K.D. Rakos; University of Vienna) - presented at General Meeting, 20th August.

An alternative to the use of a photomultiplier and moving slit aperture for extended field photometry is a solid state one-dimensional multidiode detector. A linear self-scanned photodiode array has been tested for this purpose. The detector is a linear array of 128 silicon photodiodes, each $2.5 \mathrm{~mm}$ in length and arranged on $0.025 \mathrm{~mm}$ centers with zero aperture effective dead space between them. The peak quantum efficiency is about $80 \%$ and the dark current is typically about $5 \mathrm{pA}$ per diode at $25^{\circ} \mathrm{C}$. Using a Im telescope, integration time $1 \mathrm{~s}$ and thermoelectric cooling, the difference in brightness of double stars to visual magnitude 10 and as close as 0.5 arcsec can be measured with very high accuracy. Results of UBV and uvby photometry, position angles and separations obtained with this equipment for close visual binaries have been published (Rakos et al., Astron. Astrophys. Supp1. Ser. 1982, 47, 221).

FIBRE-COUPLED CCD'S FOR COUDE SPECTROSCOPY (B. Fort; Toulouse Observatory) CCD image detectors for use in astronomy have been developed in France through work performed at Paris observatory (T. Fauconnier), Toulouse observatory (B. Fort), and the Nuclear Astrophysics Center of Saclay (L. Vigroux). The program started in 1979 with the construction of a prototype using a Fairchild 202 ( $100 \times 100$ pixels) and continued with the production of several cameras using thin back-illuminated RCA chips (see SPIE, volume 290, Solid State Imagers for Astronomy). A review of this work is given. The use of an optical fibre dissector with a CCD for coude spectroscopy is described with particular reference to performance and limitations. Measurements of the shift of the quantum efficiency bandpass at low temperatures for thick and thin CCD's are also presented, and an optimum temperature which results from a trade-off with dark current shot noise is derived for far red observations.

\section{C) Image Photon Counting Systems}

A PULSE COUNTING ARRAY DEVELOPED AT MT. STROMLO OBSERVATORY (Bruce A. Peterson) Stapinski, Rodgers and E11 is (Pub1. Astron. Soc. Pacific 1981, 93, p.242; Advances in Electronics and Electron Physics 1979, 52, p.389) have developed a two-dimensional pulse counting array (2D-PCA) which has been used for photometry and spectrophotometry. It has al so been used by Peterson and Savage for stellar calibration photometry (15-19 mag.) at the 1m telescope at Siding Spring Observatory. A large-field version of the $2 \mathrm{D}-\mathrm{PCA}$ consisting of two CCD's coupled by fiber optics to a microchannel-plate image tube is planned for operational use before the end of 1982. 
A TWO-DIMENSIONAL PHOTON COUNTER (S. Shectman; Mt. Wilson and Las Campanas Observatories)

Based on many of the same principles as the Reticon photon counters, this instrument detects photon event scintillations in a multi-stage image intensifier, using a Fairchild CCD 221 diode array. A diode readout rate of $15 \mathrm{MHz}$, and the ability to inspect as little as one-eighth of the vertical format, make possible frame repetition times as short as 1 millisecond. Photon event centers are located to 0.25 diode horizontally and 0.5 diode vertically.

PHOTON COUNTING INTERFEROMETRY AT THE 6m TELESCOPE (Michel Marcelin; Marseilles Observatory)

The Marseilles Observatory Photon Counting System has worked successfully on several large telescopes ( $2 \mathrm{~m} \mathrm{O.H.P.} \mathrm{telescope,} 3.6 \mathrm{~m} \mathrm{E.S.0.} \mathrm{telescope,} 6 \mathrm{~m}$ Soviet telescope). Interesting new results have been obtained with the $6 \mathrm{~m}$ telescope, mainly with Perot-Fabry interferometry, for the study of nearby galaxies (Boulesteix et al., I.A.U. Colloquium 67, Zelenchuk, 1981). In January 1982 we added to our I.P.C.S. a new automatically processed focal reducer equipped with a scanning Perot-Fabry interferometer. This sophisticated set of equipment is called CIGALE ("Cinematique des Galaxies") and will be used mainly at the Cassegrain focus of the $3.6 \mathrm{~m} \mathrm{C.F.H.} \mathrm{telescope} \mathrm{(first} \mathrm{obser-}$ ving run in December 1982). CIGALE will be a powerful instrument for the detailed observation of the velocity field of galaxies in Ha light. It should help in solving problems such as the density wave structure in spiral galaxies through the observation of arm-disk velocity discontinuities.

SPECTROSCOPIC OBSERVATIONS WITH MULTI-ANODE MICROCHANNEL ARRAY DETECTOR SYSTEMS (Bruce Bohannan and J. Gethyn Timothy; Sommers-Bausch Observatory, Colorado) Multi-anode Microchannel Array (MAMA) detector systems with formats as large as 256 x 1024 pixels are now being prepared for use in a series of groundbased, balloon, and sounding rocket observing programs. The modes-of-operation and the formats of the different detector systems are described briefly and high signal-to-noise spectroscopic data recorded with a visible-1ight ( 1 x 1024)pixel MAMA detector at a number of different ground-based observatories are presented. An outline of our plans for stellar spectroscopy using the $60 \mathrm{~cm}$ telescope on the University of Colorado campus is also given.

Working Group on Photographic Problems (Chairman: J.L. Heudier)

The Working Group has been very active since the Montreal meeting in 1979. The mailing 1 ist is now about 450 people, indicating the degree of interest worldwide in astronomical photography. A three-day meeting of the Working Group, held at Nice in April 1981, was attended by 80 people from 20 countries. The Proceedings of that meeting have been published (Astronomical Photography 1981 , eds. Heudier \& $\mathrm{Sim}$ ) and nearly 600 copies distributed. The Working Group has also encouraged publication of papers in the AAS Photobulletin (more than half the papers published in the Photobulletin are now from non-American contributors). Excellent contacts have been maintained with the AAS Working Group and with Eastman Kodak Company. In the period under review we have made some improvements in methods of ordering, shipping and storing plate material, and a new emulsion has become available. Kodak Technical Pan Film type 2415 is a fine grain panchromatic emulsion, similar in spectral response to the IIIa-F, but with higher resolution. The $f i l m$ version responds well to hypersensitisation but the same emulsion on glass (Kodak Special Plate Type 153-01, backed, or 153-02, unbacked) does not respond so well, at least in its initial tests. 
During the General Assembly a meeting of the Organising Committee on 18th August 1982 preceeded a business session of the Working Group and two sessions of short contributed papers. The membership of the next organising committee was adopted as follows: Chairman: Elizabeth Sim (Royal Observatory, Edinburgh, UK); Secretary: K. Ishida, (Tokyo, Japan); Members: O. Dokuchaeva (USSR), J.L. Heudier (France), H. Maehara (Japan), D.F. Malin (Australia), A.G. Millikan (USA), W.E. Schoening (USA), K.R. Sivaraman (India), A.G. Smith (USA), R.M. West (ESO, Munich) and 0. Zichova (Czechoslovakia).

The meeting noted with regret the death of W.C. Miller in October 1981, in Pasadena. Bill was a consultant to the IAU Working Group and a former Chairman of the AAS Working Group. His contributions to astronomical photography were fundamental and outstanding. The Working Group expressed its wish to be associated with the tribute to Bill which is being prepared for publication in the Photobulletin.

Seventeen papers were offered for inclusion in the working sessions. Most were presented as short contributions, and included the following: the AAS Photobulletin (A.G. Smith); hypersensitisation of $2415 \mathrm{film}$ (A.G. Smith), and of IIIa-J plates (Takase); the importance of drying hypersensitised plates (McCarthy); automatic processing of large plates (Takase); the incidence of 'gold spots' on IIIa plates (Sim); processing and storage methods to prevent gold spot formation (A. Lecart, Kodak Pathe). Descriptions were given of a new Schmidt Plate Library in Edinburgh ( $\mathrm{Sim}$ ) and the new photographic laboratories at ESO, Munich (West). Applications and research reports included an evaluation of noise on UKSTU plates digitised with COSMOS (Okamura, Tokyo); a study of granularity and resolution, grain size and pixels (Everhart, Denver); two reports on photometry with electronographic emulsions (H. Smith; Wlerick); a report of astrometry of open clusters using the Danish 1.5 metre telescope (Reiz); colour photographs of the zodiacal cloud and of Comet West (Koutchmy \& Lamy).

There is a proposal to hold another meeting of the Working Group at the Royal Observatory, Edinburgh, UK, probably in April 1984.

Working Group on High Angular Resolution Interferometry (Chairman: J. Davis)

The following papers were presented at meetings of the Working Group held on 21 st and 25th August:-

STELLAR INTERFEROMETRY AT CERGA: A REVIEW OF OBSERVATIONAL METHODS, TECHNICAL PROBLEMS AND IMPROVEMENTS (M. Faucherre)

The two telescope interferometer (I2T) at CERGA continues to obtain astrophysical data. Michelson's approach has been adopted to obtain measurements of angular diameters of giant stars: the method involves attempting to see fringes at the limit of detectability for the longest baseline so that the position of the zero of the visibility function can be determined more accurately. A simulation of $\mathrm{I} 2 \mathrm{~T}$ and atmospheric turbulence has allowed low fringe contrasts to be calibrated and observers to be trained to give improved visibility estimates. Good agreement has been found for observations of $\alpha$ Lyr. The angular diameters of five giant stars have been measured. The paths for I2T can only be matched initially to within $\pm 1 \mathrm{~mm}$ and this range has to be searched for fringes since the coherence length is only $30 \mu \mathrm{m}$ at $\lambda=0.55 \mu \mathrm{m}$. A new spectrograph is being built which will increase the coherence length to $300 \mu \mathrm{m}$. When this is completed a photon counting camera will be used in conjunction with electronics to 
compute the Fourier transform of each frame. This will give the amplitude at the fringe spatial frequency and hence the first objective measure of fringe visibility.

THE LARGE STELLAR INTERFEROMETER AT CERGA (A. Labeyrie and G. Schumacher) The aim of this project is to build an array of optical telescopes having $1.5 \mathrm{~m}$ apertures and working in coherence. Currently the first spherical mount telescope works and follows stars with \pm 1 arc second accuracy. It is able to send an afocal beam to the central station. The second telescope and the optical table where the beams will be combined are under construction and it is hoped to obtain the first fringes early in 1983.

A PROTOTYPE FOR A LONG BASELINE MICHELSON STELLAR INTERFEROMETER ( $J$. Davis) Since the completion of the programe with the Narrabri Stellar Intensity Interferometer, the Chatterton Astronomy Department of the University of Sydney, Australia, has devoted its efforts to the development of an interferometer with $\sim 100 \mathrm{x}$ the sensitivity and $\sim 100 \mathrm{x}$ the resolution of the Narrabri instrument. An amplitude interferometer, although technically more difficult than a very large intensity interferometer, promises greater sensitivity and lower cost and we are building a prototype of a modern form of Michelson's stellar interferometer. This prototype instrument is currently being installed in the grounds of Australia's National Measurement Laboratory near Sydney. It features coelostats at each end of an 11 metre baseline to direct starlight into the instrument, internal path compensation, active wave-front tilt correction and rapid signal sampling to overcome the problem of phase fluctuations. The prototype interferometer will be used to establish whether fringe visibility can be measured through the atmosphere with the reliability and accuracy required to make useful astrophysical measurements. If successful, an interferometer with baselines extending to the order of a kilometre or more is planned.

\section{A TWO-COLOR FULLY-COHERENT INTERFEROMETER FOR LARGE ANGLE AND DIFFERENTIAL ASTRONOMETRY (M. Shao)}

We report initial measurements with the NRL-MIT stellar interferometer. The instrument is a phase coherent interferometer that maintains the extreme positioning of its optical elements by the extensive use of active optics technology. The first star observed was Vega for which the angle tracking servo maintained wavef ront parallelism to 0.2 arcsec R.M.S. The fringe tracking servo maintained path length equality to better than 0.1 microns. The fringe tracker occasionally lost the fringe but the automatic fringe reacqusition algorithm was successful in finding the fringe every time (in less than 1 sec) over a period of an hour and a half without intervention by the observer. The system can be considered operational in a routine way. Data are currently being analysed to identify instrumental and atmospheric effects which must be calibrated for milliarcsecond astrometry. It is noted that there are some operational advantages of using active optics for both angular diameter measurements and astrometry. Advantages include shorter integration times, lack of ambiguities such as $n 2 \pi$ in phase measurements and no loss of temporal coherence in visibility measurements.

A MULTIPLE SPACECRAFT MICHELSON SPATIAL INTERFEROMETER (R.V. Stachnik)

A design for a multiple spacecraft Michelson spatial interferometer has been considered. The design includes two one-metre telescopes in orbits which are identical except for slightly differing inclinations. The telescopes achieve separations as large as 10 kilometers and relay starlight to a central station which has a one-meter optical delay line in one interferometer arm. Our four key findings are as follows: 1) a $1000 \mathrm{kilometer} \mathrm{altitude,} \mathrm{zero} \mathrm{mean}$ inclination orbit affords natural scanning of the $10 \mathrm{~km}$ baseline with departures from optical pathlength equality which are well within the corrective capacity of the optical delay line; 2) electric propulsion is completely adequate to 
provide the required spacecraft motions (principally those needed for repointing); 3) all necessary technology is already ${ }_{5}$ in a high state of development; and 4) resolution and magnitude 1 imits of $10^{-5}$ arcsecond and $m_{v}=15$ to 20 are achievable.

TWO APERTURE INFRARED HETERODYNE INTERFEROMETER AT CERGA ( $\mathrm{J}$. Gay and Y. Rabbia)

The infrared correlation interferometer at CERGA uses heterodyne detection, two $1 \mathrm{~m}$ aperture telescopes and operates at a wavelength of 11 microns. The instrument is still under development. Preliminary observations have revealed that the current sensitivity falls short of the estimated theoretical performance but development continues.

EXTENSION OF PHASE CLOSURE IN OPTICAL ASTRONONY (N. Arnot and A.H. Greenaway) Redundant aperture systems may be re-distributed prior to recording images in such a way that they behave as non-redundant apertures and thus allow one to use 'phase closure' to reconstruct diffraction-limited images from single turbulence-degraded frames of data. The re-distribution involves the manipulation, within an interferometer, of three sub-apertures within the redundant aperture system. The scheme will work in the high photon flux limits, extensions to lower fluxes may be possible by summing several solutions. The best signal to noise is obtained by using a $T$ shaped aperture. Preliminary reconstructions using computer simulations indicate that satisfactory solutions may still be obtained with up to $5 \%$ multiplicative noise on the data. Detailed simulations with satisfactory atmospheric models and path length correction errors etc., have yet to be done.

IMAGE RECONSTRUCTION FROM PHASELESS FOURIER DATA BY MAXIMUM ENTROPY (J. Skiliing) In general, a large number of images can be compatible with a given dataset. This difficulty is exacerbated with phaseless data, where there is a circular degree of freedom corresponding to each measured Fourier amplitude.

Maximum entropy selects from the compatible images that one with greatest configurational entropy $S=-\Sigma_{p} \log p$ (sum over pixels). This criterion corresponds to seeking a maximally non-committal answer to the question "Where would the next photon come from?", considering the image as a radiating pattern. The resulting image is uniquely free of artifacts, (and maximum entropy has been used successfully in conventional interferometry, optical deconvolution, medical tomography and el sewhere).

A program has now been written for phaseless data from 2-D images of arbitrary size [and tested up to $120 \times 120$ pixels]. Even with incomplete and noisy amplitude data, sparse fields of point sources appear to reproduce accurately. Complex objects, though, yield reconstructions which of ten differ considerably from the original. There can also be subsidiary local maxima of the entropy: amplitude data do not suffice to recover complex objects uniquely.

Future work will upgrade the program to 3-D for X-ray crystallography, and will at tempt to use maximum entropy in speckle interferometry.

Working Group on Computer Processing of Astronomical Data (Chairman: R. Albrecht)

At the meeting of the Working Group on 24 th August the Chairman's Report was read by $P$. Boyce. 
Chairman's Report

The Working Group was originally formed as an ad hoc Working Group during the 1976 IAU Meeting in Grenoble, France. Tom Bolton of David Dunlap observatory, Toronto, became the first chairman of the Working Group. The "Circular of the Working Group" was initiated to serve as a communication vehicle for the members of the group. The immediate reason for forming the Working Group was the increasing popularity of powerful minicomputers, which for many astronomers meant sudden exposure to the complex problems of sof tware development. Another strong reason was the availability to many astronomers of the PDS measuring engines and their assorted hardware and software problems.

In 1979 the Working Group was incorporated formally as a Working Group of Commission 9. The name of the "Circular of the Working Group", was changed to "Astronomical Image Processing Circular", both to streamline it a bit and to take note of the fact that the growing field of astronomical image processing was now the driving force of all non-theoretical software development. Reacting to widespread complaints that there was no adequate mechanism for the publication of sof tware and of software-related papers, efforts were made to upgrade the "Image Processing Circular" to make it attractive for the publication of such technical papers. I am sharing the editorship of the "Circular" with $M$. Capaccioli of Padova Observatory. A quarterly publication schedule was adopted and continued throughout 1979 and 1980. There followed a pause in the publication schedule during 1981 for two reasons: contributions were not forthcoming in expected numbers; and the change of affiliation from the University of Vienna to the Space Telescope Science Institute absorbed my productivity, especially during the early days of the Institute when total manpower and support were low. However, the "Circular" has been started up again and will eventually return to the quarterly publication schedule.

As already indicated, it is the purpose of the "Circular" to disseminate information related to software, both SW application and development, and especially information on a very technical level which is usually not publishable in traditional astronomical journals. In fact, even source code listings of moderate length can be published in the "Circular". The intention behind this is to allow the authors of software to get credit for it in the form of a publication, at the same time encouraging better software documentation and making the software available to the community fast. Clearly, the goal has to be more than that. Ideally there should be a software library where software and software-related information is sorted. Using state-of-the-art retrieval systems, software development can be made much more efficient through coordination and sharing. I will go into that in more detail towards the end of this report.

There are no formal rules for membership of the Working Group. Anybody with a legitimate interest in astronomical software can be a member of the Group. The membership 1 ist is identical with the mailing 1 ist of the "Circular". Since the members of the group are spread all over the world it would be very difficult for the group to engage in more technical activities. This has been taken over by local groups which have been formed recently. In Europe the "Ad Hoc Working Group for the Coordination of Astronomical Software" was formed. Chairman of this group is Phil Crane of the European Southern Observatory. In the United States the "Astronomical Software Working Group" has been formed under the auspices of the American Astronomical Society. Chairman of that group is Don Wells of the National Radio Astronomy Observatory. In cooperation with both chairmen I will now briefly report on the activities of these two groups. 
The European group has now been in existence for two years and has held several meetings hosted by major European astronomical institutes. Building on efforts by the European Space Agency to find the best approach for the development of software for the Faint Object Camera of the Space Telescope, the group extended those efforts to the global problem of astronomical software development. The major emphasis is on guidelines for software standardization and software documentation. Sof tware design issues like transportability, maintainability, and image processing techniques have been discussed, as were networks and remote processing. The "Flexible Image Transport System" (FITS), developed and maintained by Eric Greisen and Don Wells, was adopted as a data exchange standard and a FITS-compatible format has been recommended for the exchange of source code. An effort was made by myself to start up a software library but, after a promising start, this also fell victim to my move to Baltimore. I hope that I will be able to revive the project at the ST ScI although at this point I have to be careful with promises.

The main result of these activities is the awareness that software is important, crucial in fact, and that efforts to improve the quality of software and software development more than pay for themselves in the long run. Most important, people know who is doing what, and where they can get information they require. A good example is the Italian computer network ASTRONET which is currently being built up. In order to spend their money as efficiently as possible Giorgio Sedmak and his people use, wherever applicable, available solutions that have been developed elsewhere. And this, after all, is what software coordination is all about.

The AAS working group for Astronomical Software was formed later than the European Group. There have been no meetings so far but the first is scheduled for the January 1983 meeting of the AAS in Boston. Activity is expected to pick up considerably after this meeting. The objectives of the Group are to identify and propagate mechanisms and standards by which coordinated development and exchange of software will be possible. This includes coding, documentation, and data format standards. In particular, FITS will be further developed and improved. Currently, a Microprocessor Special Interest Group is being formed within the Working Group. Also, it was suggested that a "Catalog of Sites and People" be developed, and Dan Klinglesmith of Goddard Space Flight Center has volunteered to collect the data.

The first approximation to such a data base is, of course, the mailing 1 ist of the "Image Processing Circular". In October 1980 a one-page questionnaire was sent to all persons and institutes on that list requesting information on type and size of their computers, peripheral devices, software, and software systems. About $70 \%$ of the questionnaires came back and it was possible to compile some statistics. I do not want to present any numbers here because they must certainly have changed by now, but the global conclusions are undoubtedly still correct. The computer most widely used throughout the community is the DEC PDP-11. This is not as surprising as the fact that the VAX was already second in 1980. Data General Novas and HPs come next. A wide variety of other machines is used in insignificant numbers.

In the area of software it was found that all but two installations supported, and were actually using, FORTRAN, in conjunction with a vendor-supplied operating system. Several installations use non-standard operating systems like UNIX but so far the numbers are small. A remarkable result was the almost total lack of dedicated software support manpower. With the exception of some large visitor-oriented institutes, all software development is done part-time by scientists, particularly graduate students. Everybody agreed that cooperation and software sharing was desirable even though some did not think it was possible. 
Image processing was cited by the majority as the area where such collaboration was most important. This is not surprising since digital image processing is also the principal motivation for the formation of all three working groups. Microdensitometers, digital television cameras, and aperture synthesis radio telescopes are sprouting like mushrooms. The research budgets of nations and of institutes have been, and still are being, strained to provide the computing resources to process and analyze the digital imagery which these new data acquisition systems are creating. The new computer hardware demands new software, and the programming resources of institutes are also being strained to keep up with the demands made by astronomers. The people responsible for managing these programing resources naturally wish to minimize the degree to which they are 're-inventing the wheel', and so they want to coordinate the software development efforts occurring in the various institutes. There is a growing need to be able to transport software and data from institute to institute since astronomers do a lot of travel1ing and collaborating. Reports of experiences with various hardware and software components used in digital image processing systems are of great value to people who are establishing new installations. These various motivations have all contributed to the formation of the three working groups.

Despite such widespread general agreement there is not much that people seem willing to do about it. Sometimes I get the impression that software is like sex; everybody has it, needs it, but nobody talks about it. And doing it for its own sake, and maybe even improving it, is actually considered dirty. It is a fact that an awful lot of "re-inventing the wheel" is going on out there. The most scarce and most valuable resources, manpower and time, are being wasted. Also, there are some contradictions between the way in which astronomical sof tware development is treated and the treatment of other areas of astronomy; for example, although it is accepted that scientists have an obligation to publish new data and the conclusions derived from them, the same approach is not always encouraged for advances in software.

In the area of hardware development, off-the-shelf components are used widely; home-building some piece of equipment that can be obtained much easier and faster commercially is frowned upon. Yet in the area of software development we are committing all these sins. For instance, nobody publishes software and quite often it is not even communicated to colleagues working in the same institute. This is a waste of resources and should be of interest to the funding organizations. It might be worthwhile to work out some mechanism of active persuasion by which such information can be made accessible to the community.

It seems to me that what we need in the near future is some kind of software library, maybe organized in ways similar to the software libraries maintained by the user groups of some of the computer vendors. A practical solution would be to attach such a library to one of the large national institutes. This would only require adding some storage devices to existing computers and to add one, maybe two, operator-level type of people to the staff.

In Europe this is being done already by STARLINK, the British network, although the software library is intended mainly for the participants of the net. It will also be done to a certain extent by the European Coordinating Facility, the organization managing the European end of Space Telescope Science operations.

In the U.S. a similar exercise will be carried out by the Space Telescope Science Institute, this being the institute which in the long run will be the center of the biggest community of sophisticated users. Of course in both cases the emphasis will be on software useful for ST data analysis. 
This concludes my report on the activities of the IAU Working Group for Computer Processing of Astronomical Data. I would like to thank the chairmen of the two regional working groups, Phil Crane and Don Wells, for contributing material to this report.

BABSY - AN IMAGE PROCESSING SYSTEM BASED ON SOFTWARE EXCHANGE (P. Steffen, C. Crezelius, P.T. Rayner, R. Steube; Radioastronomisches Institut der Universität Bonn)

In late 1981 we started to build up the Bonn Astronomisches Bildrechner System (BABSY), based on a VAX 11-780 and an ARGS 7000 image display. In addition to our own software we have installed the following packages: AIPS (VLA), ASPIC and PICPRO (STARLINK) and GIPSY (Groningen). This work was done with the active cooperation of members of the IAU Working Group on co-ordination of astronomical software. Current work includes standards for documentation and "Online Help" and a command language structure for multiprocess control.

A NEW MICROPROCESSOR-BASED CONTROL SYSTEM FOR THE PDS-1010 MICRODENSITOMETER (H. Jenkner, M. Sto11 and J. Hron; Institute for Astronomy, Vienna) - presented at General Meeting, 20th August.

An ageing DEC PDP-12 computer, used for control of a PDS microdensitometer and for data acquisition, had to be replaced due to limited functionability, increased unreliability, and maintenance problems. The new system uses a compact Motorola Exorset-30 development system, featuring an M6809 microprocessor, 2 mini-floppy disk drives, keyboard and CRT with graphics capabilities, as well as serial and parallel interfaces. The Exorset-30 is configured to execute all control, data acquisition, and user communication tasks, whereas data storage in general formats is implemented on a DEC PDP-11/34 host computer via a serial RS-232 link. The VIPS (Vienna PDS System) software, written in Basic and assembler, provides an efficient and self-explanatory user interface (continuous display and individual input of parameters and coordinates, single key commands, argument prompting) and increased functionability (parameter/ coordinate storage and retrieval, continuous scanning, real-time graphic display during scanning, optional data transmission to host computer). 


\section{Report of Business Meeting}

PRESIDENT: V. Bumba

SECRETARY : R. Howard

A business meeting was held on August 19. The slate of new officers was approved, and the Commission elected the following organizing Committee:

President: E. Tandberg-Hanssen

Vice-President: M. Pick

Organizing Committee: A. Bhatnagar, V. Gaizauskas, E. A. Gurtuvenko, J. Machado, D. Melrose, E. Priest, K. Tanaka, and H. Zirin.

The names of new Commission members were read and approved. The Commission approved the following nominations of IAU representatives to international organizations: IUWDS, H. Coffey; QBSA, F. Moriyama; SCOSTEP, M. Kundu.

A report of the ScosTeP committee on Long-Range Planning for Future Projects was presented by $M$. Dryer. The International Heliospheric Study and Transfer of Energy within the Solar system were planned, and other suggestions were solicited.

The President read a report by L. Dezso on the efforts in Hungary to continue the Greenwich Photoheliographic Results. The first portion of this work will be published before the end of this year. There will be a study of an overlapping interval to ensure continuity. Spot polarities will be used to decide spot groupings.

A. Koeckelenberg was appointed to replace P. Simon as Chairman of the Working Group on International Programs.

\section{SCIENTIFIC SESSIONS}

1. Solar Luminosity Variations (J. A. Eddy) August 18.

H. S. Hudson: Observations of Short-Term Solar Irradiance Variations from Spacecraft.

P. V. Foukal: Interpretation of Short-Term Solar Irradiance Variations.

J. A. Eddy: Historical Reconstruction of Solar Constant Variations.

G. Frohlich: Radiometry of Solar Irradiance Variations over Long Time Scales.

J. Christiensen-Dalsgaard: Theory of Evolutionary Changes in Solar Luminosity.

W. C. Livingston: Indirect Diagnostics of Solar Irradiance Changes.

G. W. Lockwood: Luminosity Variations in Late-Type Stars.

2. Solar Maximum Year Special Session (E. Tandberg-Hanssen) August 20.

E. Tandberg-Hanssen: Opening Remarks.

C. de Jager: The SMY Idea-An Overview.

Z. Svestka: Report of FBS Activities.

D. M. Rust: Report of SERF Activities.

M. Dryer: Report of STIP Activities.

J. Smolkov/E. Stepanov: SMY Activities in the USSR.

H. Tanaka: Report on Hinotori.

K. J. Frost: Report of SMM.

M. Pick: Radio Astronomical studies During SMY. 
3. Recent Solar Results (V. Bumba) August 21.

H. Snodgrass and R. Howard: Differential Rotation of Solar Magnetic Fields.

R. W. Noyes: Magnetically Sensitive Emission Lines in the Far Infrared.

D. Ventkatesan: Solar Coronal Holes.

M. Pognerusse, C. Caroubalos, and J. Z. Steinberg: Stereo 5 Measurement of Type III and Type IIIb Radiation Patterns.

A. O. Benz: Solar Maximum Mission and Decimetric Radio Observations.

A. Dollfus: A New Solar Filter at the Meudon Observatory.

L. E. Alissandrakis: Preliminary Results from the Clark Lake Radioheliograph.

L. E. Alissandrakis: Ring Structure of an Active Region at $6 \mathrm{~cm}$ Wavelength.

T. Takakura: Sub-second Fine Structure in a Solar Burst observed Simultaneously at Hard $\mathrm{X}$-Rays and $\mathrm{mm}$ Waves.

S. Martin and P. Kaufmann: Mm-Microwave Source Outside the Solar Limb Detected After a Major Prominence Disruption.

O. F. Smith: Microwave Signature of Thick-Target Electron Beams.

E. Becklin: Recent 30, 50, 100 and $200 \mu \mathrm{m}$ Determination of the Solar Limb Extension.

K. V. Sheridan: A New Frequency at the Culgoora Radiospectroheliograph.

4. Magnetic Field Geometry in Active Phenomena.

This meeting, organized by Dr. R. G. Giovanelli, consisted of several papers on sunspot magnetic field configurations, including penumbral filaments, the heights of the horizontal field component (magnetic canopies), radio observations of quiet and active coronal fields, and airborne eclipse observations bearing on the increase of the filling factor with height above the active regions. 
COMMISSION 12: RADIATION AND STRUCTURE OF THE SOLAR ATMOSPHERE (RADIATION ET STRUCTURE DE L'ATMOSPHERE SOLAIRE)

Report of the Meetings held in conjunction with the 18th General Assembly

President : Y. Uch1da

Business Meeting of Commission 12

Business Session on August 19, 1982 was chaired by $Y$. Uchida with the secretary P. Foukal, and the following items were decided :

\section{Organizing Committee}

The Commission elected the following new Executives and Organizing Committee Members of Commission 12 for the term 1982 - 1985.

President : R. W. Noyes

Vice President : M. Kuperus

Organizing Committee Members : Chen Blao, F. Deubner, E. Fossat, J. W. Harvey, V. A. Kotov, J. Lelbacher, K. R. Sivaraman, J. O. Stenflo, P. R. Wilson, Y. Uchida ( ex-president).

\section{New Members of the Commission}

New general members of the Commission were endorsed as follows :

C. E. Alissandrakis, D. L. Book, J. B. Breckenridge, V. F. Chistyakov,

E. Chrojkova, J. W. Cook, L. Dunkelman, P. Foukal, V. Gaizauskas,

V. P. Gaur, M. V. Goldman, S. S. Hassan, R. M. E. Illing, P. Kaufmann,

S. L. Ke1l, M. R. Kundu, B. J. La Bonte, J. T. Mariska, M. R. Nicolas,

R. E. Rees, v. Rusin, M. Rybansky, J. Sakai, T. Sakurai, S. J. Schwartz,

R. Stebbins, A. H. Vaughan, J. K. Vial, D. Wentzel, L-X. Wu,

\section{Working Group on Eclipses}

Report from the Working Group on Eclipses (Drs. Leroy and Fiala) on the past and future eclipses was read by the chairman. It was decided that the Working Group is waintained with the same member in the term 1982 - 1985. Further meeting for the Indonesian eclipse in 1983 was organized separately by M. Alzenman.

\section{Resolution}

The Commission supported the following resolution :

"Comession 12, recognizing the importance of the observation of solar seismology, strongly supports international cooperation in establishing a world-wide network of observing stations. 
Scientific Sessions and Symposium Cosponsored by Commission 12

I. IAU Symposium No 102, "Solar and Stellar Magnetic Field : Origins and Coronal Effects"

This symposium sponsored by Commission 12 with the cosponsorship of Commissions $10,27,35,36$, and 44 , was held in Zurich on August $2-6$ in conjunction with the 18th General Assembly. It was organized by J. 0. Stenflo under the support of Swiss Federal Institute of Technology. Scientific Organizing Committee consisted of J. O. Stenflo ( chairman ), D. Mihalas, R. W. Noyes, A. Severny, M. Stix, Y. Uchida, G. Vaiana, N. Weiss, O. C. Wilson, and C. Zwaan, and Local Organizing Committee was headed by M. C. E. Huber. 17 invited talks and 34 contributed talks were given on the problems of the origin and the effects of the solar and stellar magnetisms. Detailed report will be published as the Proceedings of IAU Symposium No 102, separately.

\section{Joint-Discussion "Solar Luminosity Variations"}

This Joint-Discussion cosponsored by Commission 12 jointly with Commissions 10,27 , and 35 , was organized by the Scientific Organizing Committee consisted of J. Eddy ( chairman ), P. V. Foukal, D. O. Gough, G. A. Newkirk and G. W. Lockwood and was held on August 18, 1982. The program consisted of seven invited talks and six contributed talks. Details will be found in the Highlights of Astronomy.

\section{Special Session "Solar Maximum Year"}

The special session cosponsored by Commission 12 jointly with Commissions 10,40 , and 44 , was organized by E. Tandberg-Hanssen, and was held on August 20 , 1982. A whole day was devoted to the special session which consisted of ten talks summarizing the organized efforts of Solar Maximum Year Campaign including the results obtained by satellites SMM and Hinotori. Details will be found in the Highlights of Ast ronomy.

IV. Joint-Meeting "Recent Solar Results"

This joint-meeting cosponsored by Commission 12 jointly with Commissions 10 and 44, was organized by V. Bumba and was held on August 21, 1982. The description of this half-day session will be found in the report of Commission 10.

\section{Joint-Meeting "Magnetic Field Geometry in Active Phenomena"}

This joint-meeting, cosponsored by Commission 12 jointly with Commission 10, and 49, was organized by R. Giovanell1 and was held on August 23, 1982. The description of this whole-day session will be found in the report of Commission 10 .

\section{Joint-Meeting "Solar and Stellar Spots and Activity Cycles"}

This joint-meeting, sponsored by Commission 12 with the cosponsorship of Commission 10, was organized by R. W. Noyes and was held on August 24, $1982 . R$. W. Noyes opened the discussion by pointing out that recent data on spots and activity cycles of lower main sequence stars other than the sun is now providing a rich new source of information on the general phenomenon of starspots and 
stellar activity cycles. The purpose of the discussion meeting was to compare and contrast related phenomena of magnetic activity on the Sun and on similar stars. ( It was noted that the topics covered relate closely to the more in-depth proceeings of the recent IAU Symposium No 102 "Solar and Stel lar Magnetic Fields : Origins and Coronal Effects", and IAU Colloquium No. 71 "Magnetic Activity on Red Dwarf Stars").

The major content of this discussion meeting consisted of three invited review papers. First, M. Rodono reviewed "The Starspot Phenomenon", comparing physical properties of spots on BY Dra stars and RS CVn stars with those on the Sun. The speaker reviewed the observations of photometric waves on spotted stars and implications for physical properties of stellar spots and their rotation and differential rotation. Second, A. Vaughan discussed "Solar and Stellar Activity Cycles", reviewing the accumulation of data from Mt. Wilson Observatory on activity cycles of lower main sequence stars. Two principal conclusions were that solar-like cycles appear clearly only in stars with rotation periods of 20 days or more, and that for stars showing activity cycles there is at present no evidence for a dependence of cycle period upon rotation rate or on spectral type. In the third invited paper, Stix reviewed "Theories of Stellar Spots and Activity Cycles". This discussion covered the theory of concentration of magnetic flux into flux knots, and spots, as well as dynamo theories of cycles and ways in which they can be reconciled with observations of stellar cycles.

Contributed papers include : (1) H. Hudson and H. Jones ; Solar Magnetism and Rapid Variations of Solar Irradiance. (2) L. Doherty : Mg II h, k Emission in $F$ and $G$ Dwarfs. (3) A. Charles and L. Booth : The X-ray Transient 3A 1431-409. (4) P. Wilson and P. McIntosh; A New Model for the Solar Magnetic Field and its Relation to the Solar Cycle. (5) R. Howard and B. LaBonte; The Observed Relationships between some Solar Rotation Parameters and the Activity Cycle. (6) R. Giovanelli ; The Mechanism of the Sunspot and Solar Magnetic Cycles as inferred from observations. Poster presentations made during the session include : (1) A. Boesgaard and T. Simon; Observations of Chromospheric Activity through Rotation Cycles of the Young Solar-type Star $\chi^{1} O r_{1}$. (2) $\quad$ D. Dravins, P. Linde, K. Fredga, and G. Gahm; Beta Hydri ; Chromospheric Activity in a Very 0ld Solar-type Star. (3) J. Arsenijevic; A Possible Eruptive Event on the Cool Supergiant $\mu$ Cephei.

In concluding, I would like to acknowledge with the heartiest appreciation the valuable cooperation of Dr. R. W. Noyes, the Vice President, and of all the Members of the Organizing Committee, Drs. P. Delache, F. Deubner, A. Gabriel, S. Gopasyuk, J. Harvey, R. Howard, P. Wilson, and C. Zwaan, throughout the term 1979 - 1982. Finally, representing Commission 12, I would like to express our deepest sorrow for the passing away of Prof. M. K. V. Bappu, the President of IAU as of the 18th General Assembly, and the former President of Commission 12 in 1976 - 1979, and would like to deliver our heartiest condolences to his fami$1 \mathrm{y}$. 
The meeting of the Commission was opened by a brief business session, followed by a number of communtcations in a scientific session. About 30 members of the commisston attended as the meeting progressed.

\section{Bustness Session}

A. The President called attention to reprints of the triennial Draft Report, that had fust been recelved. In referring to the truncated form of some of the Working Group reports, he reminded the meeting of the plan to distribute the full reports in mimeographed form.

B. Three years ago, at the 1979 Montreal meeting, a major item of discussion was the efficacy of our current Working Groups. The only substantive results of that discussion was the change in the name of the Commisston, from "Fundamental Spectroscopic Data," to "Atomic and Molecular Data," thus opening up the possihility that the Commission might include Working Groups charged with the monitoring of important non-spectroscopic materlal, if such were deemed desirahle. However, no changes or adjitlons were made at the time. Numerous oplntons expressed at Montreal were repeated and added to. They included:

1. While historically, the current Working Groups evolved as the needs of astrophysics for data developed, and were scientifically useful in their time, the present distritution of Working Groups may not any longer reflect the current needs of astronomy for atomic and molecular data. Some Working Groups may have served their purpose and should be retired. A possible example of the latter that was discussed at Montreal was Working Group 1 (Wavelength Standards); at that time it was the consensus that it should he continued, since to astronomers good secondary standards are of vital interest, especlally when new spectral reglons become avallable to astronomical observation.

2. Perhaps some Working Groups now cover too great a field of current research and should be further suhdivided (e.g., Colliston Cross Sections and Line Broadening; Molecular Spectroscopy).

3. Perhaps other Working Groups should be added to reflect other data needed by astronomers (e.g., Thermonuclear Processes; Flementary Particle Physics). made:

During a wide-ranging discussion of these questions, the following points were

1. In their present form the reports of the Commission are not as valuable to astronmers as they might be. Many do not know of the existence of these reports, yet astronomers are always anxlous to have avallable atomic and molecular data from critically filtered data hases without the need for them to address the principal 1.terature.

2. Beyond the compllation of bibliographies of original results, an essential feature that would be welcome hy astronomers would he a critical review of data.

3. The production of such reviews is a lot of work. Probably the Trans. IAIJ is not the hest vehicle for puhlication of critical reviews. The Commission should encourage publication of such revlews 1 , say, the Annual Reviews of Astronomy and As trophysics, etc.

4. When the Commission considers other flelds (e.g., Elementary Particle 
Physlcs) to be monitored by additlonal Working Groups, it should he sure that some other Commission is not already doing so.

After consideration of the points of the discussion the meeting adopted the proposal of the chairman that:

"A subcomittee of three persons be estahlished to consider, after consultation with colleagues, which fields our Working Groups should cover, and how they should be organized. The subcomittee w11l report back hefore the 1985 General Assembly of the IAU so that their recommendations can be fully discussed at the business meeting of the Commlssion at that General Assembly."

A. H. Gabrle1, R. W. N1cholls, and K. Takayanagi were unanimously adopted as the members of this committee.

C. In a broader discussion of the service of the Commission to the astronomical community, the chalrman reported on informal discusstons he had carrted out with a number of influential astronomers. What emerged was a general consensus well-expressed by Dalgarno, that our current role of data compilation in triennial reports might he outdated, in view of the evolution of Data Centers in Europe, the US and Japan. The discussion at the meeting raised the following points:

1. The production of a loose-1eaf Commission 14 data handhook might help to keep our information current.

2. While the triennial reports already in part refer to the various data bases, they might in the future be restructured to lead astronomers more specifically to data bases of value in the various fields.

3. Data bases are all very we11, but what is also needed is a series of crttical compllations of rellable data. Fxisting data hases are of varying quality and may have been oversold.

4. What is needed is a practical and usable index of critical data hases for working astronomers.

5. Critical compilation is a hard and thankless task viewed by some as secondary scholarship, and not research. Thus few would to it as a service to the community.

6. A data base for specles of interest in stellar atmospheres is heing set up at University College London. Reference was made to the CODATA Bullet In on Atomic and Molecular Data. Few members of the Commission present at the meeting were aware of it. Reference was also made to the Berkeley Newsletter as a continually updated springboard to the literature fo molecular spectra. Phillips reported that tt accessed over 30 journals, had a malling 1 st of nearly 500 workers and had been running since 1958 .

7. There is a lack of uniformity of 1nformation 1n, and updating of, the varfous data bases and mode of access to them. What are urgently needed by the user are internationally agreed protocols for atomic and molecular data bases.

8. There is a great need for all developers and users of data hanks to establish a dialogue and reach some sort of consensus before investing a lot of time and money in such endeavors.

In the 1 ight of these comments, the chalrman suggested that the Triple Commission of Spectroscopy (IAU/IUPAC/UIPAP) be 1nformed of our concerns and he asked to help coordinate needs. Accordingly, it was moved and adopted that:

"There be a charge to the IAU representatives on the Triple Commisston on Spectroscopy to raise the profile of data banks on atomic and molecular data with their colleagues in the Triple Comission and the respective Untons to seek to achieve some sense of unfformity in data gathering and acquisition."

The current IAU members on the Triple Commission are Edlen, Phillips and Seaton. Initial informal discussions via the Triple Commission could eventually lead to more formal action by the three Unions and the involvement of the International Council of Sclentific Unlons (ICSU). It was emphasized that users 
should be consulted extensively on data needs and format. Nicholls referred to his questionnalre to be Alstrthuted to stellar spectroscoplsts on their needs for stellar spectroscoplc molecular data.

D. In connection with possthle cooperation with other Unions, the chairman reported on his discusston with Professor A. Schawlow of Stanford, the chairman of Commission 15 (Atomic and Molecular Physics and Spectroscopy) of IUPAC on possible collahoration and cooperation. Prof. Schawlow has subsequently reported general receptivity of posstble collaboration from members of his Organiz tng Committee. They will he walting to see what the astronomers suggest as an outcome of this General Assembly. Their advice and collaboration will be of great value in connection with the charge to the Triple Commission mentioned above.

F. Appointment of President, Vice-President, Organizing Committee and Cha1rmen of Working Groups for the 1982-85 period, and other committee appolntments.

President: A. H. Gabriel

Vice-Prestident: R. W. Nicholls

Organizing Committee: E. Trefftz had asked to be retired from the Organizing Committee for personal reasons. The President was instructed to send to Prof. Trefftz a letter of thanks for her service to the Commission. H. Nussbaumer was appointed to succeed her, so that the 1982-85 members on the Organizing Committee are:
D. R. Johnson
S. L. Mandel'shtam
H. Nussbaumer
S. Sahal
K. Takayanag 1
W. Wiese

J. G. Ph1111ps

It was suggested by the Incoming President, A. H. Gabrlel, that the new organizing Committee should co-opt some astronomers onto the Organizing Committee.

Chairmen of Working Groups:

$\begin{array}{ll}\text { 1. Wavelength Standards } & \text { K. M. Baird } \\ \text { 2. Atomic Transition Probabilities } & \text { W. Wiese } \\ \text { 3. Collision Cross Sections and Line Broadening } & \text { A. Dalgarno } \\ \text { 4. Atomlc Spectra } & \text { W. C. Martin } \\ \text { 5. Molecular Spectra } & \text { R. W. Nichol1s }\end{array}$

Other Memherships:

1. Consultative Committee on Definition of the Metre: A. H. Cook

2. Triple Commission on Spectroscopy: B. Edlen, .J. G. Phillips, M. J. Seaton

Commission Memberships: The Commission approved the addition of the following to the membership of the Commission:
Alan W. Irwin
D. A. Varshalovich
Ke1th Adrtan Berrington
V. S. Strelnitsky

II. Sclentific Session

1. ON A NEW DEFINITION OF THE METRE

A. H. Cook

The Cavendish Laboratory

Cambridge, ENGLAND

Professor A. H. Cook who is the representative of the Union on the Consultative 
Committee for the Definftion of the Metre of the Internat tonal Committee for Weights and Measures, described recent developments in the stabilisation and measurement of frequencles of laser radiation and the way in which they have led to the now accepted princlple that the standard of length, the Metre, should be re-defined by the distance travelled by light in a speclfled time, the speed of light being taken to be the Invarlable, convent lonal value of $299792458 \mathrm{~m} / \mathrm{s}$. The cholce of that value for the speed of 1 ight ensures that any new definition of the Metre according to those principles will be consistent with the present (1ess prectsely realisable) definition in terms of the wavelength of a line in the spectrum of $\mathrm{Kr} .86$. The Consultative Committee met in 1982 June in Sevres and adopted a resolution (of which the English text is appended) recommending a form of definition of the Metre. It is expected that the proposed new definition will be considered at the next General Conference of Weights and Measures. The defintition as proposed by the Consultat lve Committee corresponds with geophysical and astronomical practice wherehy terrestrial and astronomical distances are determined by the times of passage of electromagnetic stgnals.

\section{Append 1x}

On a New Definttion of the Metre Recommendation is 1 (1982)

The Comité Consultatif pour la Définition du Mètre, recalling its Recommendation M 2 (1979) and considering:

1 - that the present definition does not allow a sufficiently precise realization of the metre for a11 requirements;

2 - that progress made in the stahilization of lasers allows radiations to be obtained that are more reproducible and easier to use than the radiation emitted by a krypton-86 lamp;

3 - that progress made in the measurement of the frequency and wavelength of these radiations has resulted in concordant determinations of the speed of 1 ight of which the accuracy is 1 imited princlpally by the practical realization of the present definition of the metre;

4 - that wavelengths determined from frequency measurements and a given value for the speed of light have a reproductbility superior to that which can be obtained by comparison with the wavelength of the standard radiation of krypton-86;

5 - that there is an advantage, notahly for astronomy and gendesy, in maintalning unchanged the value of the speed of 1 ight recommended in 1975 by the 15 th Conférence Générale des Potds et Mesures in its Resolution 2 (c $=299792458 \mathrm{~m} / \mathrm{s})$;

6 - that a new definition of the metre has been envisaged in various forms all of whtch have the effect of giving the speed of light an exact value, equal to the recommended value, and that this introduces no appreciable discontinuity in the unft of length, taking into account the indetermination of $\pm 4 \times 10^{-9}$ of the hest realizations of the present definition of the metre;

7 - that these varfous forms, making reference elther to the distance travelled by light in a specifled time interval or to the wavelength of a radiation the frequency of which is measured or specifled, have heen the ohject of consultations and deep discussions, have heen recognized as belng equivalent and that a consensus has emerged in favour of the first type;

8 - that the Comité Consultatif pour la Définition du Mètre is now in a position to give instructions for the practical realization of such a definttion, instructions which could include the use of the orange radiation of krypton-86 used as standard up to now, and which may in due course he extended or revised.

recommends:

- that the metre be defined as follows: 
"the metre is the length of the path travelled by light in vacuum during the fraction (1/299 792458$)$ of a second";

- that the definition of the metre in force since 1960, hased upon the transition between the levels $2 \mathrm{p}_{10}$ and $5 \mathrm{~d}_{5}$ of the atom of krypton- 86 be abrogated.

\section{ATOMIC PROCESSES IN PLANETARY NEBULAE}

G. Peach

Department of Physics and Astronomy

Unfversity College London, England

A detalled knowledge of many different types of atomic process is required in order to successfully interpret the complex features observed in the spectra of Planetary Nebulag. Much progress has been made in the last few years in the development of methods that can give accurate atomic data for this purpose, and in this paper recent advances are summarised. This report is based on the talks given by Dr. C. Mendoza, Dr. P. Storey and Professor R. McCarroll at the symposium on Planetary Nebulae, held at University College London just before the General Assumbly of the IAU.

The types of atomic data required may be listed as follows:

1) Atorols Transition Probahilities

2) Rate Coefficlents for Electron Excitation

3) Photolonization Cross Sections

4) Radiative Recomblnation

5) Charge Exchange Reactions.

\section{Flectron-At om Processes}

If $A^{+m}(S L)$ denotes an $m$ times lonized atom $A$ in a state $S L$, the following processes are of Interest:

1) $A^{+m}(\mathrm{SL})+\mathrm{h} v \nRightarrow \mathrm{A}^{+m}\left(\mathrm{~S}^{\prime} \mathrm{L}^{\prime}\right)$

2) $\mathrm{A}^{+\mathrm{m}}(\mathrm{SL})+\mathrm{h} \nu \underset{\mathrm{a}}{\rightleftarrows} \mathrm{A}^{+\mathrm{m}+\mathrm{l}}\left(\mathrm{S}^{\prime} \mathrm{L}^{\prime}\right)+\mathrm{e}$

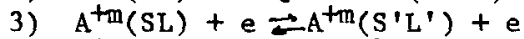

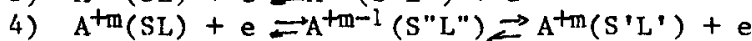

New computers, for example Cray-1, enable us to get accurate data for atoms of the 1st row and to get less accurate but nevertheless consistent data for 2nd row atoms. Calculations for heavier systems such as $\mathrm{Fe}^{+m}$ can now be attempted. A compllation of transition probabilitles, electron excitation coefficients and photolonization cross sections of interest in Planetary Nebulae has heen made and critically evaluated by Dr. C. Mendoza (University College London).

\section{Radiative Recombination}

Radiative recombination of an atomic system may take place in two ways, i.e.:

1) $A^{+m+1}\left(S^{\prime} L^{\prime}\right)+e \rightleftarrows A^{+m}(S L)+h v$

2) $A^{+m+1}\left(S^{\prime} L^{\prime}\right)+e \underset{\rightleftarrows}{\rightleftarrows} A^{+m}\left(S^{\prime \prime} L^{\prime \prime}\right) \rightleftarrows A^{+m}(S L)+h \nu$

Process 1) represents direct radiative recombination, whereas process 2) is that of dielectronic recombination, 1.e. the recombination proceeds through an intemediate doubly excited state S"L" of the atomic ion. It has been shown that the results for processes of type 1) as given by Aldrovandi and Péquignot (1973) can be wrong by as much as a factor of two or three at temperatures of $10^{4} \mathrm{~K}$ (Peach 1982, unpub11shed). Storey and Nussbaumer (1982, unpublished) have studied ions of carbon, nitrogen and oxygen and have found that the Burgess formula for dielectronic recombination grossly underestimates the effects for temperatures less thar. $10^{4} \mathrm{~K}$, because the formula neglects the effects of low-ly ing resonances. More calcalations are in progress at Unfversity College London (Peach and Storey). 


\section{Charge Exchange Reactions}

The charge exchange reactions that are important are:

1) $\mathrm{A}^{+\mathrm{m}}+\mathrm{H} \rightarrow \mathrm{A}^{+\mathrm{m}-\mathrm{l}}+\mathrm{H}^{+}$

2) $\mathrm{A}^{+\mathrm{m}}+\mathrm{He} \rightarrow \mathrm{A}^{+\mathrm{m}-1}+\mathrm{He}^{+}$

where the atom A denotes $C, N, 0$, Ne or S. Col11sions of the type 1) and 2) at thermal energies can be the dominant mechanism for determining the relative concentrations of the lons. The Inverse processes are not isually 1mportant, as $A^{t m-1}$ is usually formed in an exctted state, which rapidly decays radiatively. So far, the conclusions that have been reached are that firstly the Landau-Zener formula can be very inaccurate, and secondly that good results can he obtained for processes in which no rearrangement of the core $A^{\text {th }}$ takes place. The accuracy of the data of Butler et al. (1980) on the two-electron charge-exchange processes cannot yet be assessed (McCarro11, 1982).

\section{References}

Aldrovand1, S.M.V. and Péquignot, D.: 1973, Astron. and Ap. 25, p. 137. Aldrovand1, S.M.V. and Péquignot, D.: 1976, Astron. and Ap. $\overline{47}$, p. 321 (erratum). Butler, S.E., He 11, T.G., and Dalgarno, A.: 1980, Astron. and Ap. 89, p. 379. Mendoza, C.: 1982, "Recent Advances In Atomic Calculations and Experiments of Interest in the Study of Planetary Nebulae," Invited talk at the IAJ symposfum on Planetary Nebulae, University College London.

McCarro11, M: 1982, "Charge Exchange Reactions In Astrophysical P1asmas," invited talk at the IAU symposium on Planetary Nebulae, Universtty College London.

Nussbaumer, H. and Storey, P.: 1982, contributed paper to the IAU symposium on Planetary Nebulae.

Storey, P.: 1982, "Radiative Recombination," Invited talk at the IAII symposium on Planetary Nebulae, UnIversity College London.

\section{RECENT RESULTS ON THE SPECTRUM OF Cr II WITH ASTROPHYSICAL APPLICATIONS}

Sveneric Johansson

Department of Physics

University of Land

\section{Introduction}

An extended analysis of Cr II was initiated for special reasons besides the general purpose of lncreasing the knowledge of the atomic structure of neutral and lonized atoms. The following four points are discussed in more deta11:

(1) The structure of $\mathrm{Cr}$ II is simflar to that of $\mathrm{Fe}$ II, previously analysed by the present author (1978), due to exchange of electrons to holes in the $3 \mathrm{~d}-$ and $4 \mathrm{~s}$ shells.

(2) Iso-1onic studies of the singly Ionized 3d elements (Johansson et al. 1980 ) revealed gaps in the knowledge of Cr II, particularly for high configurations.

(3) In an article by Cowley and Arnold (1978) it was shown that many Ines in the optical spectra of Ap stars could be identified as predicted lines of Cr II, calculated from known levels, but not observed in the laboratory.

(4) There is an urgent need of $\mathrm{Cr}$ II data in the short wavelength region of IUE. In the previous analysts of $\mathrm{Cr}$ II (Kiess 1951) no lines were reported below $1780 \AA$.

A. THE ATOMIC STRUCTURE OF $\mathrm{Cr}$ II

The fact that $3 \mathrm{~d}$ - and $4 \mathrm{~s}$ electrons have almost the same binding energy means that the configurations $3 d^{k+1}, 3 d^{k} 4 s$ and $3 d^{k-1} 4 s^{2}$ have nearly the same energy. This set of configurations in $\mathrm{Cr} I \mathrm{I}$, with $k=4$, and In Fe II, with $k=6$, is given in Table 
1. These two groups of low even configurations generate the same set of LS terms, as $3 d^{7}$ and $3 d^{6}$ are equivalent to $3 d^{3}$ and $3 d^{4}$ respectively, while the presence of $4 s^{2}$ does not affect the possible values of $L$ and $S$. It can also be visualized if the three Fe II configurations are written in the number of holes instead of electrons for the $3 d-$ and $4 s$ shel1s. We then get $3 d^{3} 4 s^{2}$ (for $3 d^{7}$ ), $3 d^{4} 4 s$ (for $3 d^{6} 4 s$ ) and $3 d^{5}$ (for $3 \mathrm{~d}^{5} 4 \mathrm{~s}^{2}$ ), which is equivalent with the three electron configurations in $\mathrm{Cr}$ II.

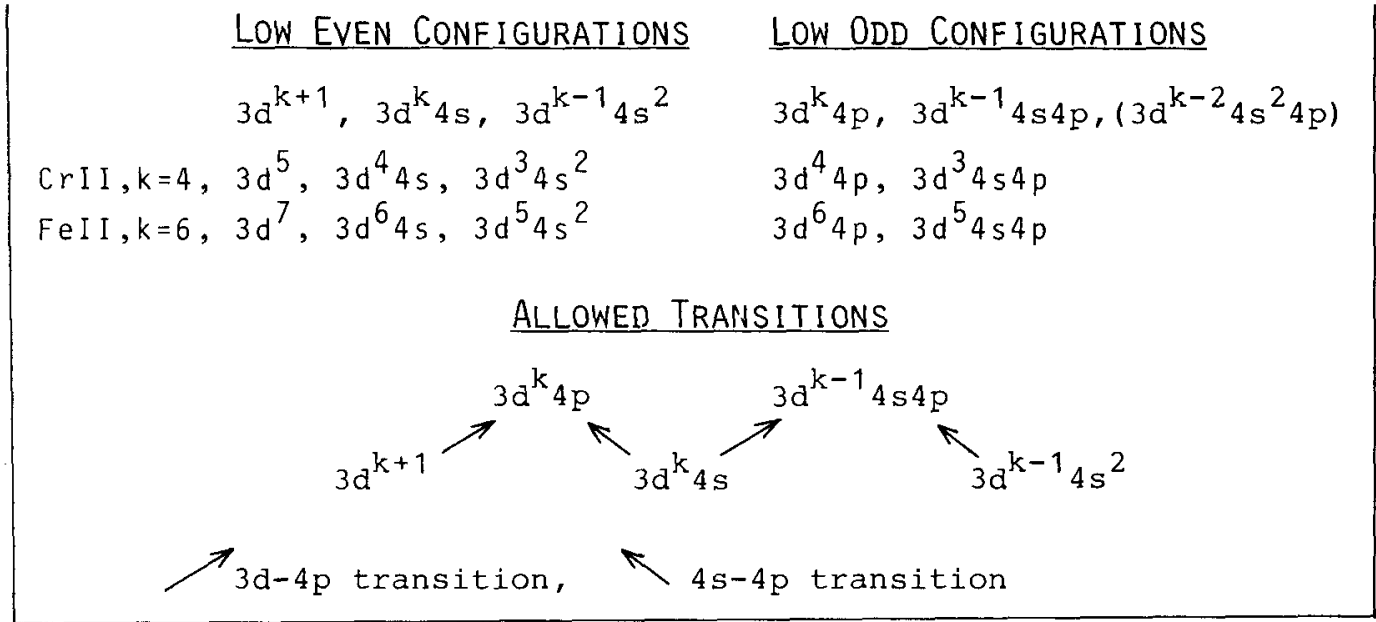

Table 1: Synoptic tahle of the low configurations in the spectra of neutral and singly fonlzed iron group elements. The strongest transition arrays are isplayed.

The overlapping of these three low configurations of the same parity is constierable through the whole iso-tonic sequence of singly ionized iron group elements. This is illustrated in Figure 1 where the relative posttions of the configurations are represented by thelr lowest LS term. Together these configurations generate a great number of metastable leve1s, 73 in $\mathrm{Cr}$ II and 61 in Fe II, and thereby a large set of possible forbitien transitions. The increasing binding energy with $k$ of the $3 d$ electron relative to the $4 \mathrm{~s}$ electron in the two halves of the $3 \mathrm{~d}$ shell allows $3 \mathrm{~d}^{\mathrm{k}+1}$ to constitute the ground state in five cases and $3 d^{k} 4 \mathrm{~s}$ in four cases, while $3 \mathrm{~d}^{\mathrm{k}-1} 4 \mathrm{~s}^{2}$ never gets this status in second spectra. When a $3 \mathrm{~d}-$ or $4 \mathrm{~s}$ electron is exclted to a $4 \mathrm{p}$ orbital we get three odd conflgurations, as 11lustrated in Table $I$, but the third one, $3 \mathrm{~d}^{\mathrm{k}-2} 4 \mathrm{~s}^{2} 4 \mathrm{p}$, is predicted to appear high up in the level system and is generally not observed. The strongest absorption Iines in the second spectra of the Iron group elements are consequent1y due to $3 d-4 p$ and 4s-4p trans1tions, which normally appear below $3000 \AA$ (see Figure 5).

\section{B. ISO-IONIC REGULARITTES}

The iso-ionic regularities through the sequence of singly tonfzed iron group atoms provide possibilities to roughly predict the positions of the lowest terms in different configurations. In Figure 2 the term value, 1.e. the absolute value of the binding energy, is plotted for the lowest level of the observed configurations. From this graph the position of the completely unknown configuration $3 \mathrm{~d}^{3} 4 \mathrm{~s} 4 \mathrm{p}$ in Cr II was interpolated and the strongest transitlons down to $3 d^{4} 4 \mathrm{~s}$ were predicted to appear around $1430 \AA$. This is the most prominent group of 1 ines below $2000 \AA$ and $i t$ will be discussed further tn Section III. 


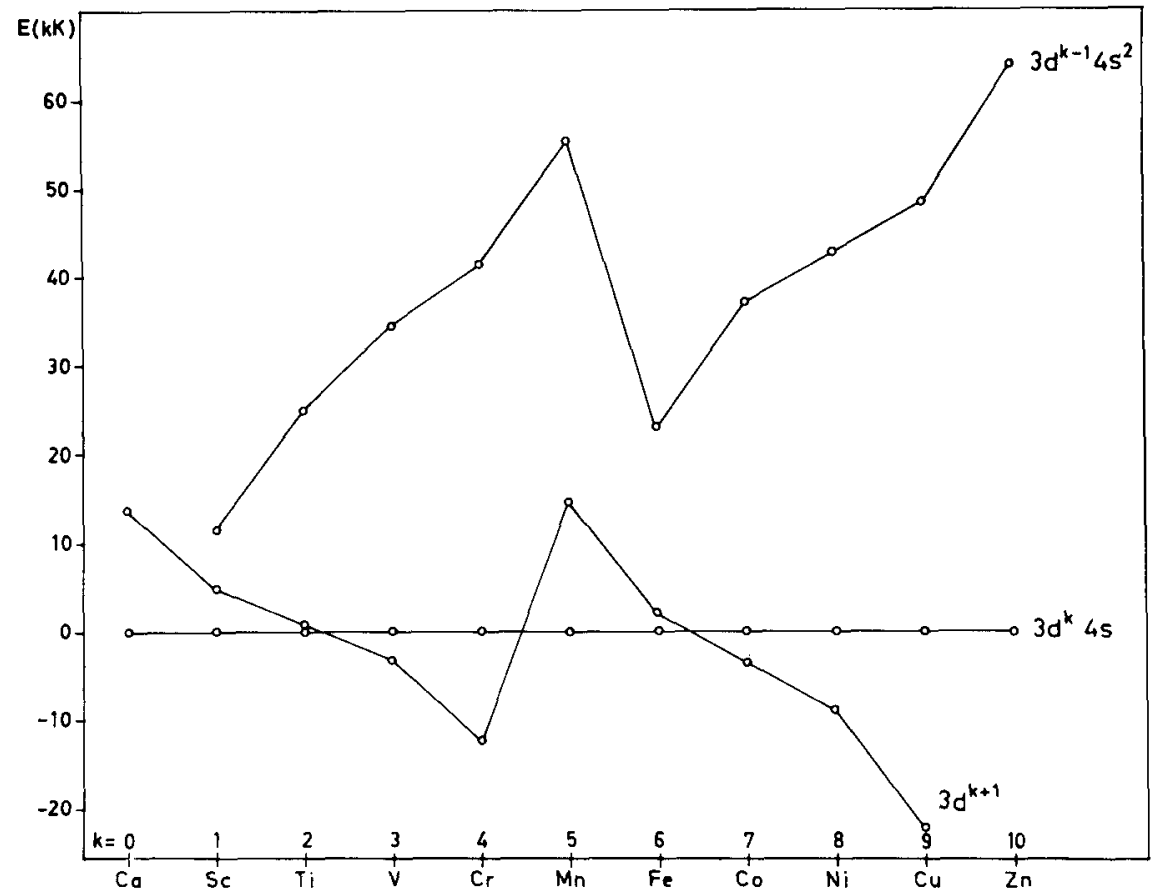

Fig. 1: Positions of the lowest terms of the $3 \mathrm{~d}^{\mathrm{k}+1}$ and $3 \mathrm{~d}^{\mathrm{k}-1} 4 \mathrm{~s}^{2}$ configurations relative to the lowest term of $3 \mathrm{~d}^{\mathrm{k}} 4 \mathrm{~s}$ for singly fontzed iron group elements.

\section{PREDICTED Cr II LINES IN STELIAAR SPECTRA}

The reinvestigation of neitral and singly ionized iron group elements by means of hollow cathode lamps have offered better possihilities to separate and analyse the spectra. The additions to the term structure have often been extracted from new ohservations in the vacuum ultraviolet and the infrared reglons in the later studies. In some cases the line rich optical region (3000-5000 $A$ ) has not been reexamined, whtch means that the old 1 ine lists are still valid for this region. Most of the old analyses were based on recordings of the spectrum from an arc in air with serlous blends with molecular bands as well as other tmpurity 11 nes. However, in most cases the high quality of the analyses is impresslve, and the very few mistakes in the prevlous work on e.g. Fe II and Cr II give evidence for that. These analyses were carried out wthout the support of parametric calculations of the term structure, which is an important tool in modern spectral analysis. It should be ment toned that the data complied in the Revised Multiplet Tahles (Moore 1945) for the neutral and singly lonized iron group elements are extracted from these analyses from the 1920s and 1930s. From this background it is not surprising that many IInes, calculated from later level compllations give rise to new itentifications in stellar spectra. Thus, the important suggestion by Cowley and Arnold (1978) that doubtful Identifications may be replaced by permitted transitions in fron group spectra 1llustrate the continuous need of updating all useful comptlat lons and tables. Cowley will illustrate this tdentffication technique by some spectfic examples at the Joint Commission Meeting and his article together with Arnold as well as his talk elucldates the need of more laboratory spectroscopy of elements with high cosmic abundances. 


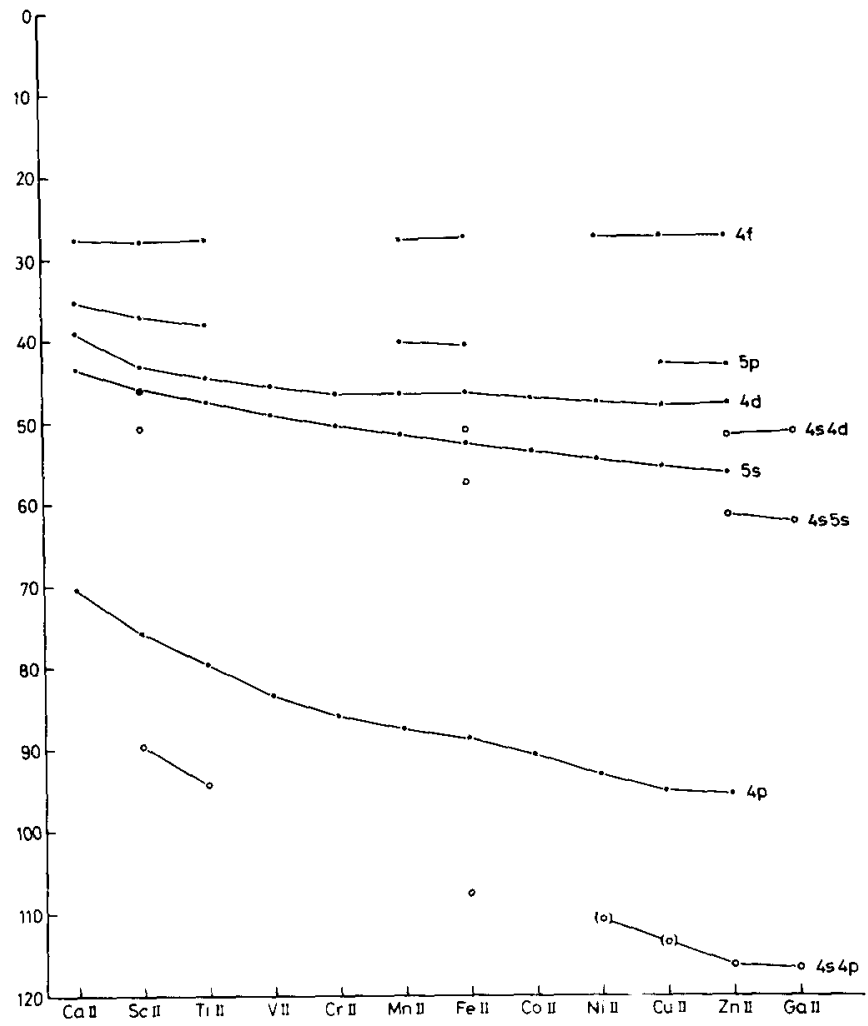

Fig. 2: Term values of the lowest-1imit levels with highest possible

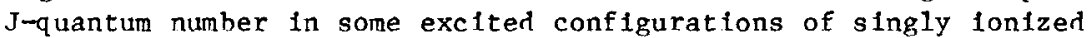
Iron-group elements (from Johansson et al. 1980).

\section{Cr II DATA IN THE IUE REGION}

The work by Kiess on Cr II (1951) does not include any lines below $1780 \AA$, which explains why the $3 d^{3} 4 \mathrm{~s} 4 \mathrm{p}$ configuration was unknown. In the present work about 1000 lines have been identified below this earlier limit. The self-evident fact that Iines at lower wavelengths and higher energies are assigned to transitions from deep levels in the energy diagram give them hlgh probability to contribute to the opacity in stellar spectra.

\section{Experiments and Results}

\section{A. RECORDINGS OF DATA}

The Cr II spectrum has been recorded in the vacuum ultravlolet region with the $10.7 \mathrm{~m}$ normal incirence spectrograph at the National Bureau of Standards, Washington D.C., whch has an Inverse dispersion of $0.78 \mathrm{Amm}^{-1}$. The advantage of using that instrument has become evtdent during the analysis of the spectrum. As the $3 d^{5}$ terms theoretically (to the first approximation) should have no fine structure the observed splittings of the terms were expected to be very small. That means a need of high dispersion in order to resolve the components of a LS multiplet. Take a 4 P in $\mathrm{Cr}$ II as an example, where the two lowest levels are separated by only $0.3 \mathrm{~cm}^{-1}$, which corresponds to a wavelength ifference of $0.01 \AA$ at $2000 \AA$ and $0.03 \AA$ at $3000 \AA$. Another example is a very narrow LS multiplet at $2330 \AA$, UV 47 . The small fine structures of the combining terms, two quartets, gather all the ten 1 ines in the multiplet within $1.4 \AA$. Plates in the air region have heen taken at Iund and 
Madrid. In Figure 3 we can see how the separation of different lonization stages is possible on hollow-cathode spectrograms recorded with a stigmattc instrument. The figure contains two spectrograms of Fe II taken under different conditions of the 1 ight source, the top one is from a continously burning hollow cathode lamp and the bottom one from a pulsed discharge. The distribution of the light inside the cathode is projected on the slit and the stigmatic image of the llluminated slit on the plate preserves the distribution in the shape of the vertical 1 ine.
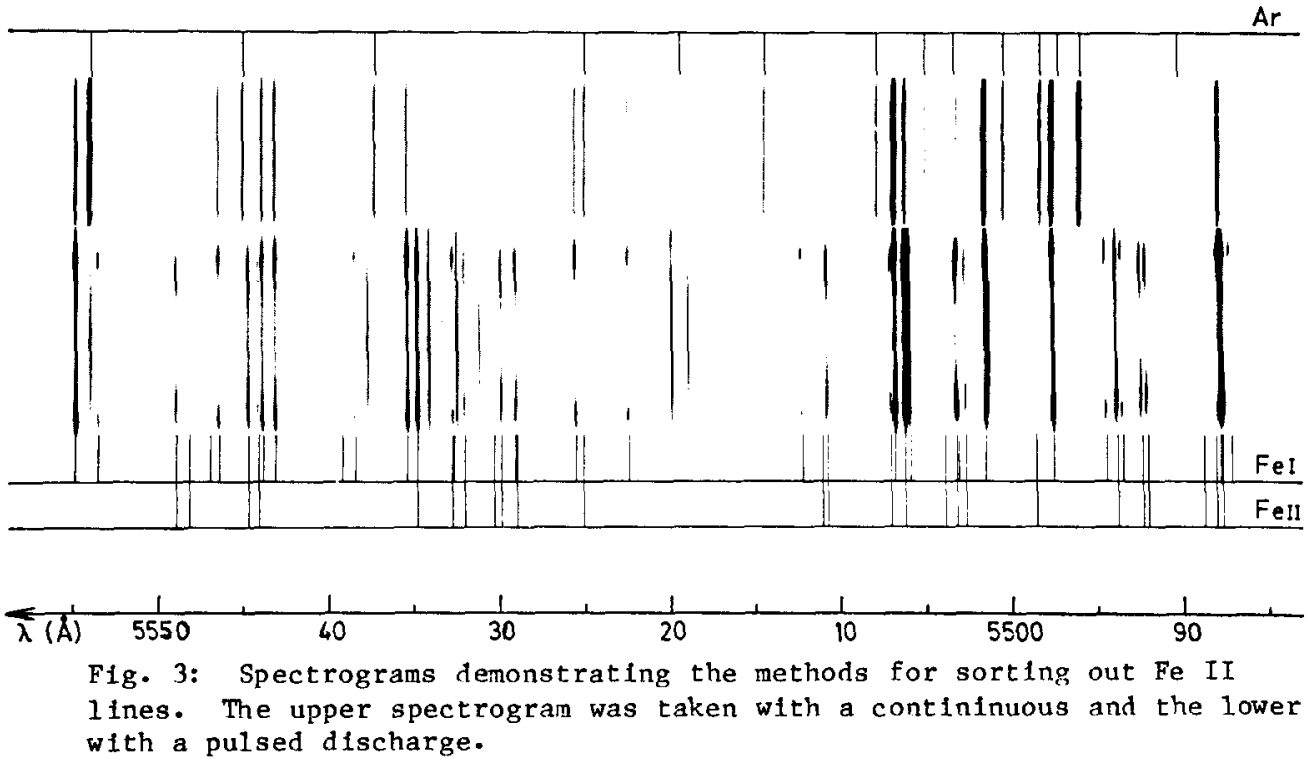

B. RESIJLTS

The results of this investigation will eventually be presented in long tables of wavelengths and level values. To give an idea of the quantity of data some statistics are given in Table 2. Even if the number of known levels is now about 600 there is only one completely known configuration, namely $3 \mathrm{~d}^{5}$ with 37 levels.

Table 2

Condensed Data for Cr II

\begin{tabular}{ll}
\hline Number of measured spectral lines & $\simeq 15,500$ \\
Number of 1ines classifled as Cr II & $\simeq 6,000$ \\
Number of identified Cr II Iines & $\simeq 4,900$ \\
Number of unfientified Cr II lines & \\
with an intensity of $>1$ & $\simeq$ \\
Number of known Cr II levels & $\simeq 600$
\end{tabular}

In Figure 4 is given the distribution of known levels of $\mathrm{Cr} I I$, where the hatched areas represents the additions from the present investigation. The sharp cut-off at $110 \mathrm{kK}$ may be due to the excltation conditlons in the 1 ight source or to the decreasing transition probabilities for lines from highly exclted levels. The level system is falrly complete up to $100 \mathrm{kK}$ as only some 30 levels are missing below that value. In the energy range $100-110 \mathrm{kK}$ about 150 levels are still unknown but predictable from parametrlc calculations. The configurations $3 \mathrm{~d}^{3} 4 \mathrm{~s} 4 \mathrm{p}$ and $3 d^{4} 4 d, 5 d, 4 f, 5 p$ account for the major part of the hatched area in the histogram. 


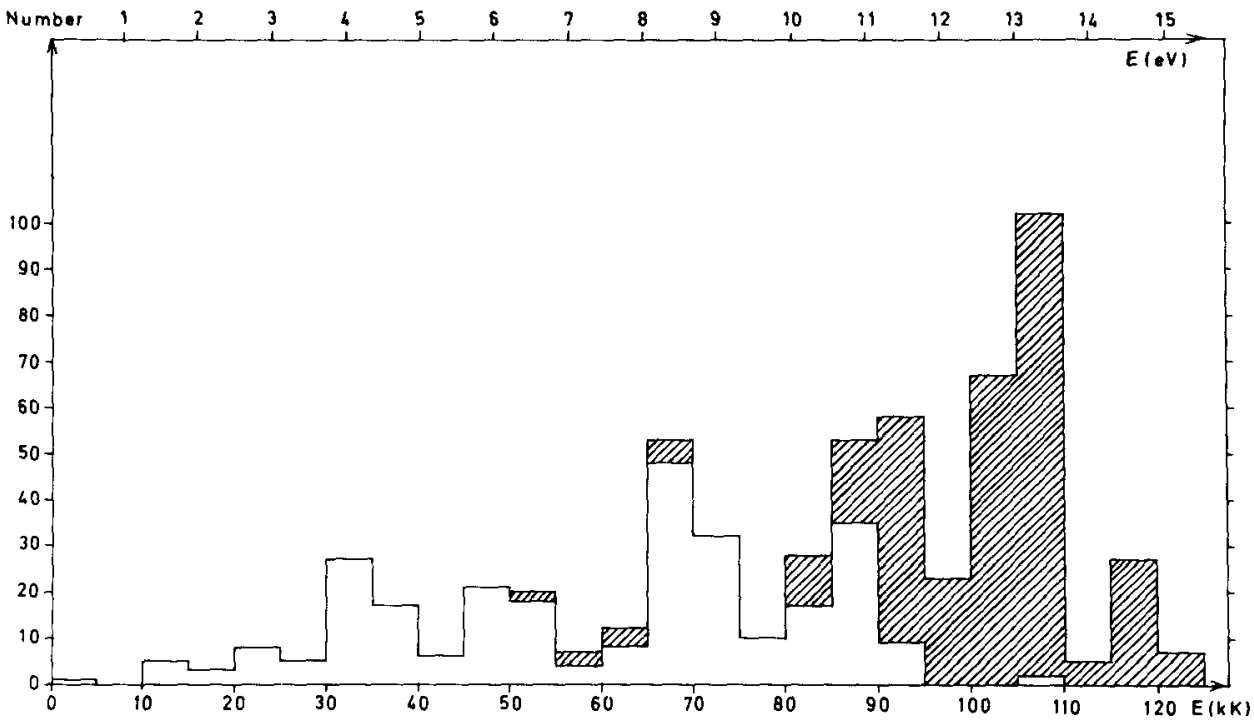

Fig. 4: The distribution of known levels in $\mathrm{Cr}$ II as a function of energy. The hatched area represents the additions from the present investigation.

The spectral distribution of the observed Iines is illustrated in Figure 5, where the number of Identified lines in certain wavelength intervals is represented by the area of a box. The upper $11 \mathrm{mit}$ of wavelength is set by the investigation itself, while the lower $1 \mathrm{imit}$ is set by the radiating atoms in the 1 ight source. We also infer from this histogram that the major part of the transitions appear in the IUE region. The regions where the strongest radiation occurs from different transition arrays are indicated below the histogram.

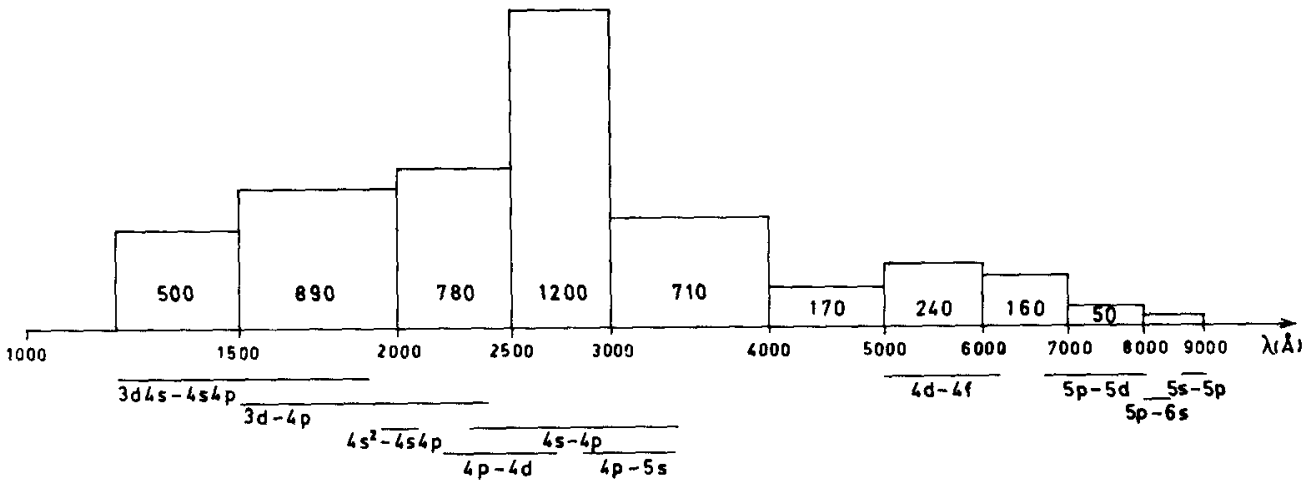

F1g. 5: The spectral distribution of the tientified lines in $\mathrm{Cr}$ II. The number of 1 ines in each box is represented by the area. Below is indicated the reglons, where the different transitions occur.

\section{SELECTIVE POPULATION}

When the light source is run under different conditions the excitation and ionization rates are drastically changed. This is 11lustrated in Figure 6, which 
shows a part of the spectrum around $2000 \AA$, produced under different $d$ ischarge modes. The upper one is from a pulsed discharge with a peak current of $80 \mathrm{~A}$ and a repetition of $100 \mathrm{~s}^{-1}$ and the lower one is from a continuously burning discharge with a current of $0.8 \mathrm{~A}$. Norma1ly the $\mathrm{Cr}$ II spectrum is much stronger in a pulsed discharge, while the continuous discharge favours the spectrum of the neutral atom. However, on these spectrograms almost all lines are due to $\mathrm{Cr} I \mathrm{I}$, even those on the low current spectrogram. The strong multiplet, which is indicated on the lower spectrogram, is generated from selectively excited $\mathrm{Cr}$ II levels. The excitation potential of these levels is about $14.5 \mathrm{eV}$ and they are populated through a charge transfer reaction between $\mathrm{Ne}^{+}$and $\mathrm{Cr}$. When the lonization energy of $\mathrm{Ne}^{+}$is transferred in a collision the neutral $\mathrm{Cr}$ atom will he fonized and left in a highly excited state. This process will consequently affect the spectrum from the low-energetic 1 ight source, which normally contains 1 ines only from the neutral atom and probably the resonance lines from the singly lonized spectrum. Th 1 s can be seen in Figure $G$ as well, where the 1 ines marked $R$ are resonance transitions in $C r$ II and they appear much weaker than the high level transitions in the low current ischarge.

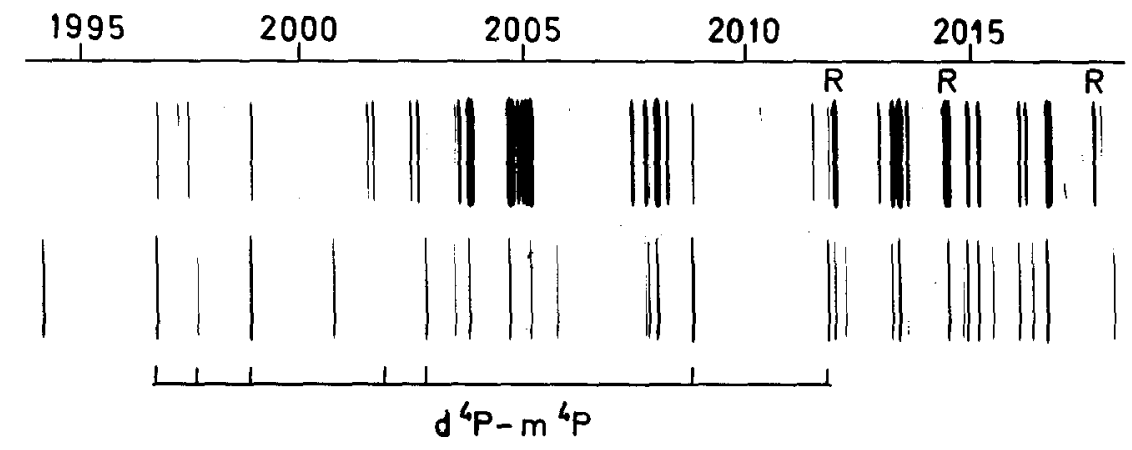

Fig. 6: Spectrograms demonstrating selective excltation of high levels of Cr II due to charge transfer. The upper spectrogram (pulsed discharge) contains three resonance lines (R), which are weak on the lower spectrogram (continuous discharge). The upper term in the indicated multiplet, $\mathrm{m}^{4} \mathrm{P}$, with an E.P. of $14.5 \mathrm{eV}$ is selectively populated in the continous discharge.

\section{Astrophysical Applications}

The high abundance of Cr II in some CP stars has made it meaningful to compare the laboratory spectrum with stellar spectra. For instance, Cowley has supplied me with a line list of HR 4816, arranged hy Bidelman, and in the reglon $3700-4800 \AA$ all identifled laboratory Cr II lines are present in the spectrum of this star. There are also traces in the stellar spectrum which might be identified as $5 p-6 \mathrm{~d}$ transitions with a lower EP of about $11 \mathrm{eV}$. If such lines noticeab1y contribute to the opacity, then the corresponding transitions in Fe II would appear due to the normally higher abundance of iron. However, these lines are not known in Fe II and cannot be predicted since the $6 \mathrm{~d}$ configuration $1 \mathrm{~s}$ still unknown. This is the case in most singly ionized iron group spectra and the occurence of such high level transittons would imply a neef of extensions of most existing analyses. In this last section we will come hack to the prominent group of lines around $1430 \AA$ mentioned in section IB. Recently "An At las of Solar Spectra in the VUV Region Recorded on SKYIAB" was issued by Cohen (1981). In Figure 7 is shown a part of the spectrum from a solar active reglon, where a group of strong absorption lines appear. On the bottom of the flgure the strong Cr II group is displayed on the same wavelength scale. There is a perfect agreement between the absorption features in 
the solar spectrum and the two $\mathrm{Cr}$ II multiplets. It should further be mentioned that all identified Fe II and N1 II Iines in the spectrum of this active region appear in emission and that some more $\mathrm{Cr}$ II lines show up as weak absorption features. A more detalled description of this coincidence is described in a paper submitted to Monthly Notices (Johansson 1982).
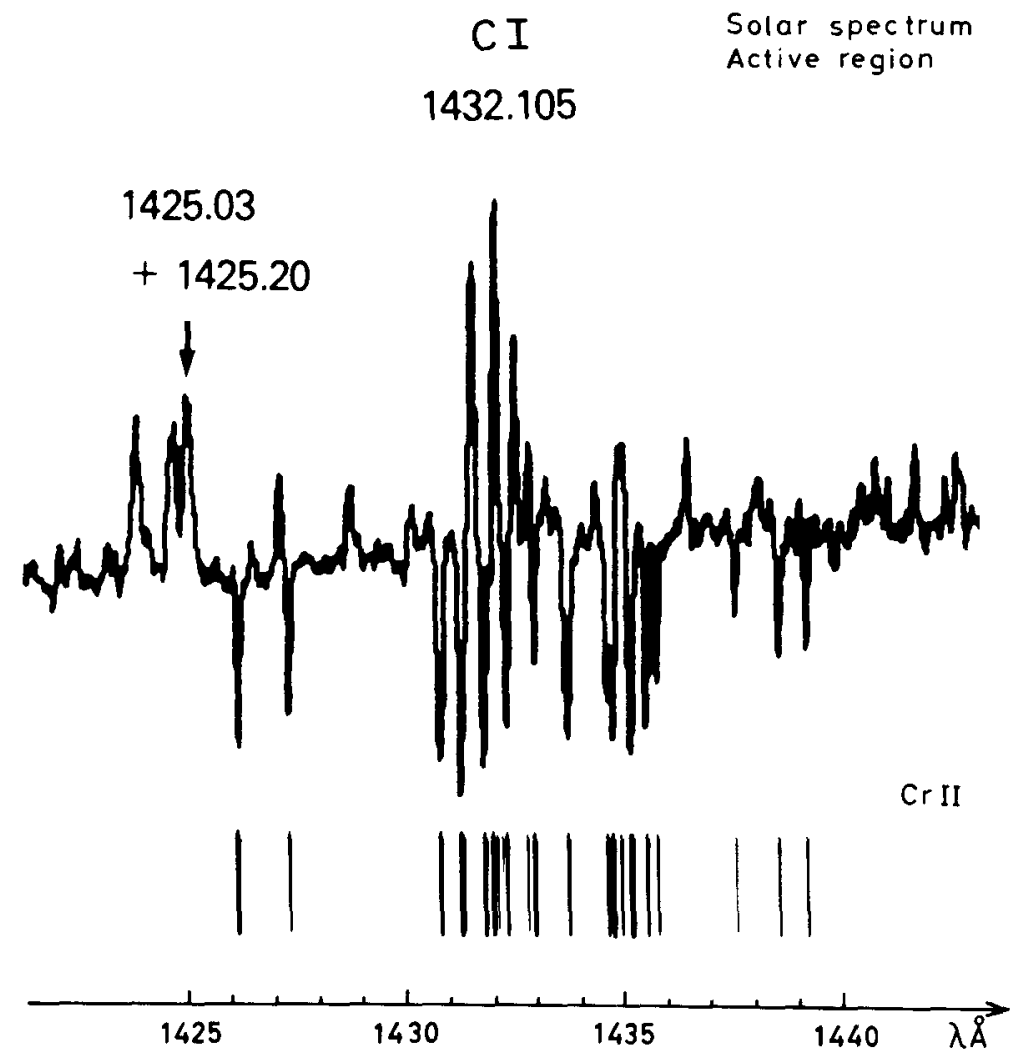

Fig. 7: A part of the spectrum of an active region on the sun, extracted from an atlas of solar spectra (Cohen 1981), compared to the laboratory spectrum of $\mathrm{Cr}$ II.

\section{References}

Cohen, L.: 1981, NASA Reference Publication 1069.

Cowley, C.R. and Arnold, C.N.: 1978, Ap. J. $\overline{226,}$. 420.

Johansson, S.: 1978, Phys. Scr. 18, p. 217.

Johansson, S., L1tzén, J., Sinzelle, J., and Wyart, J.-F.: 1980, Phys. Scr. 21, p. 40 .

Johansson, S.: 1982, Mon. Not. Roy. Astron. Soc., in press.

Kiess, C.C.: 1951, J. Res. Nat. Bur. Stand. 47, p. 385.

Moore, C.E.: 1945, Contrib. Princeton Ohs. No. 20. 
4. DATA BASES FOR ASTROPHYSICAL MOLECULAR SPECIES

R. W. Nicholls

York Un 1versity, Canada

\section{Introduction}

The impacts of laser phenomena on research in chemical physics together with increasing demands on spectroscopy for dlagnostic tools in atmospheric remote sensing have led in recent years to greatly increased research activity in molecular spectroscopy. This activity has been in both of the major research areas of a) determination of structure constants, and b) 1ine profile parameters. Relatively few molecular spectroscopy research laboratorles devote much of their attention to spectra solely of astrophysical importance.

It is impossible to do justice in this report or the draft report, to the very $v$ igorous research activity of the past three years, on many aspects of the spectroscopy of diatomic molecules. The vigour of the flelit is clear from examination of the many Iiterature citations in each lssue of the well known and Invaluable Berkeley Newsletter [1].

Even a cursory survey of the recent critical compllation "Spectroscople Constants" by Huber and Herzberg [2] reveals many gaps in the structure constant data for electronic states of astrophysically important diatomic molecules.

Astrophysical and other users cannot always waft unt 11 these data gaps are f11led. Two tasks should thus be undertaken, perhaps at the instigation of Comission 14. They are:

a) To develop approximate methods for the interpretation of those molecular astrophysical spectra for which rellable data are sparse.

b) To develop internationally accesstble and easy-to-use data bases of critically complled constants of astrophysical molecules. The compllations should be presented in formats most useful to the astrophysical community. It is anticlpated that thermochemical as well as molecular structure and transition probability constants would be included in such data bases. They would allow for the straightforward computation of synthetic molecular spectra of astrophysical importance.

The difficulties of Implementing these tasks, particularly the second, should not be underestimated.

The first task presents some interesting theoretical challenges, which can be Individually tackled [3].

Progress on the second task is made more difficult by the perhaps mistaken perception of many individual research workers that the development of critical compllations of data, no matter how useful and badly needed such compllations are, is an activity of "secondary scholarsh1p," and not therefore as attractive as primary research. The tasks involved in critical assembly of continualiy updated data compllations are enormous and very time consuming. To undertake them in an orderly fashion calls for we11-coordinated distribution of effort among many collaborators. The total effort will be best divided among a number of easily identifled (and publishable) sub-tasks, each of whlch is complete in itself.

The more experlenced the research workers who are Involved in making the compllations, the more rellable and authorftative they will be.

\section{Compllations and Data Bases}

Numerous comptlattons do of course exist to ald the astrophysical user of 
diatomic molecular spectroscopy. They include the regular 1 isting of 1iterature references in the Berkeley Newsletter [1], the 1istings of principal features of many hand systems In Pearse and Gaydon's Indispensible Identifications of Molecular Spectra [4], supplemented by the CNRS Donnees Spectroscoplque edited by Rosen [5], Suchard's Spectroscoplc Data [6], the varfous collections by Krupenie et a1. of spectroscopic data for individual molecules [7], the compllation of Huber and Herzberg [2], the 1 ine 1 ists on $C N$ and $C_{2}$ spectra of PhI111ps et a1. [8,9], and the York Unlversity series of "Identification Atlases of Molecular Spectra."

None of these absolves the astronomical user from making an Individual assessment of the primary 11terature.

Except for the work of Ph1111ps et al. [8,9], no ordered compllations of rotational ine identifications, and Iine intensities appear to be available for astrophysical diatomic molecular spectra comparable to, for example, the AFGL 1 ists by McClatchey et al. for $\mathrm{CO}_{2}$ and other atmospheric molecules [10]. This continua11y updated data base Is also avallable on computer-compatthle tape for those who need to use the data for truly realistic studies of atmospheric transmission.

We work in an environment in which computer-accesstble data bases of many kinds are becoming increasingly more avallable tools for research. Typlcal examples are:
a) Iiterature search data hases
b) the IANAF Thermochemical data base
c) the AFGL Atmospheric Molecules data base
d) the Unfv. Sussex Molecular Potentlals data base

Some of these (e.g. (a)) are accessible on a "user-pay" basis. Others, (e.g. (b)) are published in hard cover and provide for many species a continuously updated set of thermochemical data tables. Yet others, (e.g. (c)) are avallable, by subscription, on computer tape. And others, (e.g. (d)) may be accessed through remote terminals.

Of these, Item (c), the AFGL data base on positions and strengths of 1 ines of atmospheric polyatomic molecules, such as $\mathrm{CO}_{2}$, which contribute to atmospheric opactiy in the infrared and ricrowave reglons of the spectrum, is a good model for a data hase of astrophysical molecular spectra. More spectral information should undoubtedly be contalned in the astrophysical data base.

III. An Astrophys1cal Mo lecular Spectrum Data Base

In the context of the above comments, it is proposed to 1 imtt this discussion to data hase needs for electronic spectra of diatomic molecules of astrophysical interest in general, and in particular to those of Importance to stellar spectrocopy.

To Identffy the optimum contents and format of a data base on molecular contributions to stellar spectra, the user communty, and molecular spectroscopists interested in stellar spectra should be invited for comments on:

a) What wavelength range (waveband)?

b) Which molecular species?

c) Which spectral features of these spectes?

d) Which molecular (and other) data on these specfes?

e) What data accuracy would he acceptable?

f) What data presentation format data is most useful?

g) What presentation medium is most useful?

h) What are the priorities comments on each of these?

Astronomers have access to different instruments and will thus be intrested in different spectral wavebands. They work on ifferent astronomical objects and thus 
are interested in different molecular species, and different spectral features of the species. They also work at different resolutions. Some need line information. Some would be content with band data. Different problems call for different molecular data. Some problems call for 1 ine positions. Some call for 1 ine and band strengths and related transition probability data. Others call for 1ine profile information. Different problems also call for fundamental data at different precisions.

There is probahly a wide spectrum of opinions on optimum data display formats. Similarly because of the many media on which data are commonly displayed (hard copy, computer tape, microcomputer diskette, etc.) there is 11kely to be a wide spectrum of opnions on the best media on which to make parts of the data hase available.

Answers to the above questlons by a representative number of astrophysical users of molecular data would provide a most valuable indication of the optimum content of an Astrophysical Molecular Spectra Data Base. It is hoped that during the coming year, Working Group 5 (Molecular Spectra) can gather this information from at least Commission 27 (Stellar Spectra). Later, a wider group of potentlal users might be polled. On the basis of this information, it is hoped that Commission $14 \mathrm{might}$ be able to encourage the collaborative and cumulative provision of an Astrophysical Molecular Spectrum data base, perhaps one band system at a time.

The minimum data which it is anticipated would be provided for each band system are:

a) line 1ist (band and branch number identifications)

b) assoclated wavelengths and wave numbers

c) Iine strength (Hon1-Lonion Number)

d) Iine widths under typical stellar atmosphere conditions

e) band Franck-Condon Factors and r-centroids

f) band system electronic transition moment

g) band system molecular structure constants

h) typical synthetic spectra

1) principal references

In astronomical applications, where spectral features of a number of molecular species are interlaced in a particular observational waveband, parts of the band-system data bases could be appropriately merged by users if display formats and media are chosen with care.

It is hoped that this proposal might be considered by interested members of the astronomical and spectroscoplc community before the next meeting of Commission 14.

\section{References}

[1] Phill1ps, J.G., Davis, S.P., and Eakin, D.M.: Berkeley Newsletter, Departments of Astronomy and Physics, Intversity of Calffornia, Berkeley, California.

[2] Huher, K.P. and Herzberg, G.: 1979, Constants of Diatomic Molecules, Van Nostrand Re Inhold, New York.

[3] Nichol1s, R.W.: 1982(a), Ap. J. Supp1. 47, pp. 279-290. 1982(b), J. Quant. Spect. Rad. Transf. (in press). 1982(c), J. Chem. Phys. 77, pp. 1614-1616.

[4] Pearse, R.W.B. and Gaydon, A.G.: 1976, The Identification of Molecular Spectra, (4th Edition), Chapman and $\mathrm{Ha} 11$, London.

[5] Rosen, B. (Ed.): 1970, Spectroscopic Data Relative to Diatomic Molecules, Pergamon Press, Oxford.

[6] Suchard, S.: 1975(a), Spectroscopic Data: Volume I - Heteronuclear Diatomic Molecules, (Part A), Plenum, New York. 1975(b), Spectrocopic Data: Volume I - Heteronuclear Diatomic Molecules, (Part B), Plenum, New York. Suchard, S. and Meltzer, J.E.: 1976, Spectroscopic Data: Volume II Homonuclear Diatomic Molecules, P1enum, New York. 
[7] Krupenie, P.H.: 1972, J. Phys. Chem. Ref. Data 1, No. 2.

[8] Davis, S.P. and Philitps, J.G.: 1963, The Red System of the CN Molecule, Un1versity of Calffornia Press, Berkeley, CA.

[9] Phillips, J.G. and Dav1s, S.P.: 1968, The Swan System of the $C_{2}$ Molecule and the Spectrum of the $\mathrm{HgH}$ Molecule, Untversity of California Press, Berkeley, CA.

[10] McClatchey, R.A. et a1.: 1973, AFCRL Atmospheric Absorption Line Parameters Compilation, ATFR $\overline{\mathrm{L}-\mathrm{TN}}-73-0096$.

\section{Append 1 $\mathrm{x}$}

The following questionnaire was distributed at the scientific sesston. It is hoped to distribute an updated version of 1 t by mail to interested parties during 1982 and 1983.

COMMENTS ON NEEDS FOR AN ASTROPHYSICAL MOLECIULAR SPECTROSCOPIC DATA BASE

1) Which molecular species are most important to you?

2) Which band systems, bands, or Iines of these species are most important?

3) Which wavelength ranges or wavebands are of principal and subsidiary importance?

4) What types of molecular data are most needed for your work?

5) What is the preferred format and medium (hard copy, tape, diskette, other) for these data?

6) Comments you wish to make?

Please send comments by air mall to:

Professor R. W. Nicholls

Centre for Research In Experimental Space Sclence

York University

4700 Keele St reet

Downsview

Ontario, CANADA M3J $1 \mathrm{P3}$

5. THE MOLECULAR SPECTROSCOPY FILE AT JPL

D. D. Locanthi

Jet Propulsion Laboratory

About 14 years ago the Planetary Atmospheres Section at JPL (Jet Propulsion Laboratory), at the Instigation of Dr. C. B. Farmer, began maintalning in a systematic manner a file of papers about molecular spectra. Only those molecules, radicals, and lons that are found (or expected to be found) in the atmospheres of the planets or comets of the solar system are Included in the 1 ist, which grew from about 115 to 345. More recently molecules found in interstellar space were added.

For each paper two information cards have been typed and kept in one section of the M.S.F. (Molecular Spectroscopy File) one glving the author's name on the top Ine, the other having the accession number at the top. A coding system on the cards provides access to information descrtbed in the articles: molecular constants, processes, experimental conditions, etc. The papers themselves are arranged in order of an accession number. About thirty articles are added per month. There are now about 6000 papers in the file.

To facllitate searching for papers about glven molecules, the information on the typed cards has been transferred to standard punched cards. For each paper there is first a title card, which shows accession number, name of first author, abbreviated title, year of publication, volume number, page, and journal of publication. In the beginning, the list of journals was alphahet1c, but, as new 
journals were added, alphabetization could no longer be mafntalned. A second punched card for each paper gives the code numbers that correspond to information in the article. A program for searching and listing all papers that our flle contains for a given molecule was written by H. Aumann; but it is seldom used because of the expense involved in computer search. Instead a small file is kept with a card for each molecule/radical/ion. Whenever a new paper is added to the system its accession number is typed on the appropriate card. Thus, if one wants a 1 ist of a11 papers on $\mathrm{HC} 1$, for example, one may simply pull the punched cards for all papers noted on the $\mathrm{HCl}$ card, and have the computer 1 ist them.

The future of the M.S.F. is a little uncertain, owing to drastlc decrease in funds allocated to NASA. Though our section's concerns are now necessarily 1 imited to molecules found only in the Earth's stratosphere, we are attempting to continue Including papers about all molecules in the solar system and interstellar space.

\section{ADDITIONS TO REPORT OF WORKING GROUP 2 (ATOMIC TRANSITION PROBABILITIES)}

John Black

Center for Astrophysics

Harvard College Observatory

The Atomic and Molecular Physics Division of the Harvard-Smithsonian Center for Astrophysics has constructed an ion trap and is using it for the first laboratory measurements of A-values for the Intersystem lines of 1 ight fons that are used in the diagnosis of astrophysical plasmas. Many of these lines have A-values of the order of $10^{2} \mathrm{sec}^{-1}$ to $10^{4} \mathrm{sec}^{-1}$ and none have been measured. As a consequence, astronomers have used unconfirmed calculated values until now.

Preliminary results for the SI III $3 s^{2}{ }^{1} s_{0}-3 s 3 p{ }^{3} P_{1}{ }^{\circ}$ Iine at $1892 \AA$ give $A=(1.63 \pm 0.26) \times 10^{4} \mathrm{sec}^{-1}$. This value is in good agreement with the calculations of Cowan et al. (1982) and Laughiln and Victor (1979), but is smaller than the results of Nicolas (1977), $2.25 \times 10^{4} \mathrm{sec}^{-1}$. Our measurements are being described at IAU Colloquium 73 by Parkinson, Kwong, Sinth and Johnson (1982).

Similar measurements for the ${ }^{4} D-2 P^{\circ}$ multiplets of $C$ II and Si II at $-2320 \AA$, for the ${ }^{1} S-{ }^{3} P^{0}$ line of $C$ III at $1909 \AA$, and for the ${ }^{3} P-{ }^{5} S^{\circ}$ multiplet of $O$ III at $1660 \AA$ are underway.

Electron impact excltation cross sections are being measured with inclined electron and ton beams at this laboratory by G. P. Lafyatts and J. L. Koh1. The near threshold cross section for excttation to the upper state of the C II $\lambda 1335$ \& spectral Iine was measured from threshold to $14.5 \mathrm{eV}$ and measurements of the well-established cross section for C IV $\lambda 1550 \AA$ have veriffed the experimental methods being used (Lafyatis, 1982). A measurement of dielectronic recombination in C IV is currently in progress.

\section{References}

Cowan, R.D., Hobbs, L.M., and York, D.G.: 1982, Ap. J. 257, p. 373.

Lafyat1s, G.P.: 1982, "An Experimental Study of Electron Impact Excitation of Positive Ions," Ph.D. Thesis, Harvard U.

Laughlin, C. and Victor, G. A.: 1979, Ap. J. 234, p. 407.

Nicolas, K.R.: 1977, "The Application of S1 III Line Intensity Ratios to Determine the Solar Transition Zone Density and Pressure," Ph.D. Thesis, U. of Maryland. See also, Nicolas et al.: 1979, Ap. J. 233, p. 741.

Parkinson, W.H., Kwong, H. S, Smlth, P.L., and Johnson, B.C.: 1982, "Measurements of A-values for Intersystem Lines Us ed in Diagnosis of Astrophysical P1asmas," to be presented at IAU Colloquium No. 73, Dublin, Ireland, August, 1982. 


\section{FRANCK-CONDON FACTORS AND SYNTHETIC PROFILFS FOR MOLECULES OF ASTROPHYSICAL INTEREST}

Basile Petropoulos

Research Center for Astronomy and Applted Mathematics

Athens Ac ademy, Greece

Plasma spectroscopy is a good diagnostic tool to study the chemical reactions in the plasma of the sun of comets, atmospheres of planets and in interstellar s pace.

This method is consisting of the comparison of the computed synthet ic profiles with the measured, absolute intensities obtained by spectrophotometric methods. For this comparison it is possible to determine plasma parameters, such as the concentration and the temperature of the molecular plasma. The following data are needed for the computation of synthetic profiles: 1) Hönl London factors; 2) Franck-Condon (F.C.) factors; 3) electronic transition moments; 4) measurements in laboratory as Spectroscopical constants, dissociation energies, 1ifetimes of electronic states, oscillator strengths.

Franck-Condon factors have been computed used a Morse potential to resolve the one dimension Schrödinger equation, for the most diatomic molecules of astrophysical interest by McCa1lum et al. [1].

Different authors have used the more correct R.K.R. Cooley method to compute F.C. of diatomic molecules of astrophysical interest, Petropoulos [2], Sp11nder [3], and Petropoulos et a1. [4,5]. A rotational effect has to be taken into account for the computation of $\overline{\mathrm{F} . C}$. factors, at high temperatures. This effect is more important for molecules of type XH. Consequently F.C. factors are needed for the following transitions: $\mathrm{CH}^{+}(\mathrm{A}-\mathrm{X}), \mathrm{MgH}(\mathrm{A}-\mathrm{X}), \mathrm{MgH}^{+}\left({ }^{1} \Sigma-1 \Sigma\right), \mathrm{AlH}(\mathrm{A}-\mathrm{X}), \mathrm{AlH}^{+}(Z I I-Z \Sigma)$, $\mathrm{SiH}^{+}(A-X), \operatorname{PH}\left({ }^{3} \Pi-{ }^{3} \Sigma\right), H S(A-X), \operatorname{CaH}(A, B, C-X), \operatorname{FeH}(A-X), \operatorname{CoH}(A-X), N i H(A, B, C-X)$, $\mathrm{BaH}(\mathrm{A}-\mathrm{X})$, $\mathrm{T} 1 \mathrm{H}(\mathrm{A}-\mathrm{X})$. As also for the following transitions of molecules: $\mathrm{MgO}(\mathrm{B}-\mathrm{X})$, $S c O(A, B-X), \operatorname{YO}(A-X), \operatorname{ZrO}(A, B, C-X), T 1 O(B-X, b-d), V O(A-X), S 1 O(A-X)$ and $C N(\operatorname{Red}$ and Ballik Ramsay transitions).

For polyatomic molecules the computation of F.C. factors is more difficult. Different methods have been proposed to integrate the multidimensional Schrödinger equation. The method of generated functions has been used by Sharp and Rosenstock [6], which have computed F.C. factors for $\mathrm{CO}_{2}, \mathrm{C}_{2} \mathrm{H}_{2}, \mathrm{C}_{2} \mathrm{D}_{2}, \mathrm{CS}_{2}$. An harmonic potential has been admitted by these authors to resolve the Schrobdinger equation. The normal coordinates $Q^{l}$ of the upper electronic state of the molecule, can be obtained by the following orthogonal transformation of the normal coordinates $Q$ of the lower electronic state for each symmetric vibration:

$$
Q^{l}=/ J / Q+/ K /
$$

where $/ \mathrm{J} /$ is a rotation matrix and $/ \mathrm{K} /$ a transition matrix. The rotation effect was pointed out by Duchinsky [7] (Duchinsky effect). Lucas [8] has observed that this effect is only a first order approximation, because for the Eckart's [9] axes system the transformation of coordinates is not orthogonal.

In Sharp-Rosenstock [6] computations the Duchinsky effect has been taken into account, in its first approximation given by relation (1). The method has been applied to compute F.C. for $\mathrm{H}_{2} \mathrm{O}, \mathrm{H}_{2} \mathrm{O}^{+}$and $\mathrm{NH}_{3}, \mathrm{NH}_{3}{ }^{+}$, by Botter et al. [10] for small or large molecules of hydrocarbons by Warshel et al. $[11,12]$ and for Benzen by Metz [13].

Coon [14], Craig [15] and other authors have used the one dimenslonal method to compute F.C., but they have not taken into account the Duchinsky effect. 
Doktorov [16] Introduced another method to compute F.C. of Benzen, as also Faulkner [17], where Duchinsky effect is also taken into account in its first approximation. Petropoulos $[2,18]$ proposed a general theory which takes into account the Lucas [18] transformations of coordinates and the one dimension theory to resolve the Schrödinger equation. Morse functions or R.K.R. potentials can be used for computing F.C. factor for the symmetric vibrations of every molecule. This method has been applied to compute F.C. factors of the electronic transitions:

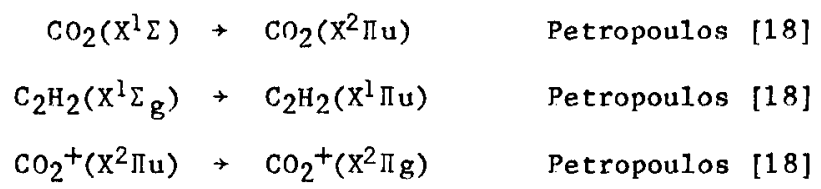

and also for

$$
\mathrm{H}_{2} \mathrm{O}\left(\hat{\mathrm{X}}^{1} \mathrm{~A}_{1}\right) \rightarrow \mathrm{H}_{2} \mathrm{O}^{+}\left(\hat{\mathrm{X}}^{2} \mathrm{~A}_{1}\right) \quad \text { Petropoulos }[2]
$$

F.C. factors computed by this method are in good agreement with the measured values of transition probabilities, and the Computed F.C. factors by Botter et al. [10]. McCallum [19] have also computed F.C. factors for $\mathrm{CO}_{2}$ and $\mathrm{CO}_{2}^{+}$by used Morse potential. For these 1 inear molecules Duchlnsky rotation effect can be neglected, Petropoulos [2].

Polyatomic molecules have been observed in spectra of Interstellar medium and comets as $\mathrm{H}_{2} \mathrm{O}, \mathrm{HCO}, \mathrm{H}_{3}^{+}, \mathrm{HCO}^{+}, \mathrm{C}_{2} \mathrm{H}, \mathrm{HCN}, \mathrm{HNC}, \mathrm{N}_{2} \mathrm{H}, \mathrm{H}_{2} \mathrm{~S}, \mathrm{OCS}, \mathrm{SO}_{2}, \mathrm{HNO}, \mathrm{CH}_{2}, \mathrm{CO}_{2}$, $\left(\mathrm{CO}_{2}+, \mathrm{H}_{2} \mathrm{O}^{+}, \mathrm{NH}_{2}, \mathrm{C}_{3}\right.$ : comets only) $\mathrm{H}_{2} \mathrm{CO}, \mathrm{NH}_{3}, \mathrm{HNCO}, \mathrm{H}_{2} \mathrm{CS}, \mathrm{C}_{3} \mathrm{~N}, \mathrm{HCOOH}, \mathrm{HC}_{3} \mathrm{~N}, \mathrm{CH}_{2} \mathrm{NH}$ (comets only), $\mathrm{NH}_{2} \mathrm{CN}, \mathrm{H}_{2} \mathrm{CCO}, \mathrm{HCH}_{2} \mathrm{OH}, \mathrm{NH}_{2} \mathrm{CHO}, \mathrm{CH}_{3} \mathrm{CN}, \mathrm{CH}_{3} \mathrm{C}_{2} \mathrm{H}, \mathrm{CH}_{3} \mathrm{CHO}, \mathrm{HC}_{5} \mathrm{~N}_{2} \mathrm{CH}_{3} \mathrm{NH}_{2}$, $\mathrm{CH}_{2} \mathrm{CHCN}, \mathrm{CH}_{3} \mathrm{COOH},\left(\mathrm{CH}_{3}\right)_{2} \mathrm{O}, \mathrm{CH}_{3} \mathrm{CH}_{2} \mathrm{OH}, \mathrm{CH}_{3} \mathrm{CH}_{2} \mathrm{CN}, \mathrm{HC}_{2} \mathrm{CN}$. And in the atmospheres of planets: $\mathrm{N}_{2} \mathrm{O}, \mathrm{NO}_{2}, \mathrm{CO}_{2}, \mathrm{H}_{2} \mathrm{O}, \mathrm{H}_{2} \mathrm{~S}, \mathrm{COS}, \mathrm{SO}_{2}, \mathrm{O}_{3}, \mathrm{~S}_{3}, \mathrm{CO}_{2}+$. For the above molecules Franck-Condon factors are needed in order to compute synthetic spectra by the Whiting et al. [20] program which has been applied for diatomic molecules, by Danylewych et al. [21] for the Swan hands of $\mathrm{C}_{2}$. These authors by comparing their results with absolute spectrophotometric measurements of the Swan bands of the comets (1973) (XII) Kohoutek and 1975 IX (Kabayask1-Berger-Milon), have determined one relationship between the molecular column density and the electronic excitation temperature. Macris and Petropoulos [22] have also used the above program to compute the synthetic profile of the $C O\left(A^{l} \Pi-X^{l} \Sigma\right)$ transition in low and high temperatures. The computed intensities for low temperatures have been compared to absolute intensities measured in the Mars atmosphere by the Mariner's 9 spectrometer. From this comparison, the distribution of $C O$ has been obtained, for different altitudes.

The computed profiles for high temperatures and concentrations, have been compared to the measured absolute intensities, of the sunspots, observed by the telescope of Sky-Laboratory. From this comparison they have obtained the minimum solar temperature.

\section{References}

[1] McCallum, J.C., Nicholls, R.W. and Jarmain, W.R.: 1970, "Franck-Condon Factors and Related Quantities for Diatomic Molecular Spectra," Spectrosc. Rep. Cent. Res. Exp. Space Sciences, York Unfversity.

[2] Petropoulos, B.: 1974, These doctorat of Sc lences, Paris VI, No. CNRS 3216.

[3] Splinder, R.J.: 1965, J. Quant. Spectr. Rad. Transfer 5, p. 165.

[4] Petropoulos, B., Dessaux, 0., Chaffiol, D., Goudmand P.: 1967, Com. Rend. Acad. Sc 1., 265, pp. 355-358.

[5] Petropoulos, B. and Herman, L.: 1967, Com. Rend. Acar. Sc 1. 264, p. 250.

[6] Sharp, T.E. and Rosenstock, H.M.: 1964, J. Chem. Phys. 41, p. 3453. 
[7] Duchinsky, F.: 1937, Acta Physicochim. U.R.S.S. 7, p. 551.

[8] Lucas, N.J.D.: 1973, J. Phys. (B) 6, p. 155.

[9] Eckart, C.: 1935, Phys. Rev. 47, p. 553.

[10] Botter, R. and Rosenstock, H. M.: 1969, J. Res. Nat. Bur. Stand. Sect. A73, p 313.

[10a]Botter R., Diheler, V.H., Walker, J.A., and Rosenstock, H.M.: 1966, J. Chem. Phys. 44 (3), p. 1275.

[11] Warshel, $\overline{A .}$ and Karplus, M.: 1975, J. Chem. Phys. 62, p. 214.

[12] Warshe1, A.: 1975, J. Chem. Phys. 62, p. 214.

[13] Metz, F., Robey, M.J., Schlay, E.W. and Dorr, F.: 1977, Chen. Phys. Lett. 51, p. 8.

[14] Coon, J.B. and De Wanes, Loyd C.M.: 1962, J. Mol. Spectr. 8, p. 825.

[15] Cra1g, D.P.: 1950, J. Chem. Soc. 2146.

[16] Doktorov, E.V., Ma1kin, I.A., and Marka, V.I.: 1975, J. Mo1. Spectr. 56, p. 1; 1977,64, p. 302 .

[17] Faukner, T.R. and Richardson, F.S.: 1979, J. Chem. Phys. 7n, p. 1201.

[17a]Faukner, T.R.: 1979, J. Chem. Phys. 71, p. 2737.

[18] Petropoulos, B. and Botter, R.: 1968, Com. Rend. Acad. Sc1. 266, p. 104; 266, p. 276; 266, p. 933.

[19] McCallum, J.C. and Nichol1s, R.W.: 1972, J. Phys. Atom. Mo1. 4, p. 1096; 5, p. 1417.

[20] Whiting, E.E.: 1972, Ph.D. thesis, York University, Toronto.

[20a]Whiting, F.E., Paterson, J.A., Kovaks, I., and N1cholls, R.W.: 1973, J. Mol. Spectrosc. 47, p. 84 .

[20b]Whiting, E.E.: NASA Tech. Note 57268.

[21] Danylewych, I., Nichol1s, R.W., Neff, J.S., and Tatum, J.: 1978, Icarus 35, p. 112 .

[22] Macr1s, C. and Petropou1os, B.: 1982 (1n press), Com. Rend. Acad. Sci., Proc. of the 6th European Congress of I.A.U., Belgrade, 1981. 
COMIISSION 15: PHYSICAL STUDY OF COMETS, MINOR PLANETS AND METEORITES (L'ETUDE PHYSIQUE DES COMETES, DES PETITES PLANETES ET DES METEORITES)

Report of Meetings, 20,21,24 and 25 August 1982

PRESIDENT: B. DOnn

SECRETARY: J. Rahe

20 August 1982

BUSINESS MEETING

The meeting of the Commission opened with noting the deaths of Past President N. Richter and A. E. Douglas and holding a brief period of silence in respect. The serious illness of Vice-President $B$. Levin was also noted. Because of this illness, $\mathrm{Dr}$. Levin could not accept the nomination as President and with regret his name was withdrawn.

The Trienniel Report of the Commission was distributed. Because of the late receipt of the Soviet contribution by 0 . V. Dobrovolsky it was distributed separately.

\section{Election of Officers, New Members and Consultants}

President:

V. President

Organizing Committee:

New Members:

Consultants :
Clark R. Chapman

L. Kresak

B. Donn, F. Dossin, H. Fechtig, M. Festou, D. L. Matson, Z. Sekanina, H. V. Schmidt, L. M. Shul'man, J. Wasson, G. W. Wetherill, L. Wilkening

E. Barker, W. Cochran, B. Cosmovici, J. Crovisier, J. C. Degewij, P. Farinella, J. A. Fernandez, M. Gaffey, D. M. Gibson, J. Gradie, E. Grun, D. W. Hughes, R. Hutchinson, W. H. Ip, W. Irvine, S. Isobe, K. Jockers, A. C. Levasseur-Regourd, D. F. Lupishka, S. M. P. McKenna-Lawlor, R. Newburn, P. Pellas, D. Revelle, M. Shimizu, H. Takeda, E. F. Tedesco, P. R. Weissman, H. Tanabe, T. Nakamura

F. Begemann, G. Briggs, V. A. Bronshten, K. Churyumov, M. Combi, P. Eberhard, H. Hoopes, Z. Knezevic, G. Kurat, S. Larson, M. Oppenheimer, K. Saito, P. Signer, G. Turner, R. Zerull

\section{Resolutions}

Two resolutions were approved by the Commission membership for submission to the General Assembly. One called for IAU recognition of the International Halley Watch and the other requested observatories to give high priority to selected Comet Halley observations. These were adopted by the General Assembly and appear under resolutions of the General Assembly.

\section{Working Group Reports}

Filter standards. M. A'Hearn described the status of the program to develop and make available standard sets of filters for cometary photometry. To obtain a set of these filters contact Dr. M. F. A'Hearn, Astronomy Program, University of Maryland, College Park, Maryland, 20742, USA. 
Comet Filter Specifications

diameter $-2.54 \mathrm{~cm} \pm 0.05,(1.000 \mathrm{in})$, thickness $<0.95 \mathrm{~cm}(0.375 \mathrm{in})$

\begin{tabular}{|c|c|c|c|}
\hline Species & $\lambda_{0}$ & FWHM & Peak Tran \\
\hline $\begin{array}{l}\mathrm{C}_{2} \\
\mathrm{coont}_{\text {ont }}\end{array}$ & $\begin{array}{l}5139 \\
4845 \pm 10\end{array}$ & $\begin{array}{l}90 \pm 10 \\
65+10\end{array}$ & $\begin{array}{l}0.50 \\
0.50\end{array}$ \\
\hline $\mathrm{C}_{3}$ & $4060 \pm 5$ & $70(+10,-5)$ & 0.40 \\
\hline $\mathrm{CN}$ & 3871 & $50(+0,-10)$ & $>0.20$ \\
\hline Cont. & $3650 \pm 10$ & $80(+10,-5)$ & $>0.25$ \\
\hline
\end{tabular}

\section{Additional Filters}

$\begin{array}{lll}\mathrm{OH}^{+} & 3085 & \\ \mathrm{CO}^{+} & 4623 & 45 \\ \mathrm{Cont}^{+} & 7500 & 80 \\ \mathrm{H}_{2} \mathrm{O}^{+} & & \end{array}$

Fifteen sets have been distributed, another twenty-five have been assigned, five are available and additional sets are on order. There is a charge of $\$ 335$ per set of five. This will provide funds for expanding the set. The initial purchase was made with a grant from the U. S. National Science Foundation. This enables the cost to be subsidized for observers who do not have funds to purchase the filters.

Cometary Archives. The Notebooks of $\mathrm{N}$. Bobrovonikouv are being prepared for publication by A. H. Delsemme. These are expected to be ready before much longer.

The preparation of an Atlas of Comet Halley, 1910, by J. Rahe, B. Donn and J. Brandt is well underway. PHotographic collections from the Cordaba, Harvard, Heidelberg, Helwan, Kodaikanal, Lick, Lowe11, M. Wilson and Yerkes observatories were made available. These included exposures made in Hawail. The set for inclusion in the Atlas have been selected and prepared.

Workshop on Modern Observational Techniques for Comets. The Workshop was organized by J. Brandt, B. Donn. J. M. Greenberg and J. Rahe and held at Goddard Space Flight Center, October 22-24, 1980. The proceedings of the meeting have been published as JPL Publication 81-68. Copies may be obtained from Dr. J. C. Brandt, Code 680, Goddard Space Flight Center, Greenbelt, Maryland 20771, USA.

Atlas of Cometary Spectra. Work on the updated version of the Swings and Haser "Atlas of Representative Cometary Spectra" by C. Arpigny, B. Donn, F. Dossin, J. Rahe and $\mathrm{S}$. Wyckoff is continuing. Enlarged reproductions of essentially all spectra with dispersions greater than $20 \mathrm{~A} / \mathrm{mm}$ have been prepared. Medium resolution spectra of several of these comets will be included to facilitate the interpretation of such spectra by comparison with high dispersion observations. In addition to photographic reproductions, tracings of spectra will be presented. With the increasing trend to digitized spectra using electronic detectors, example of such data will be shown. Examples of ultraviolet and near infrared spectra are planned. Tables of identified species and wavelengths and unidentified lines will be included.

Coordinated Comet Observing. Plans under development in this area have effectively been taken over by the comprehensive International Halley Watch. Detailed accounts of the IHW were distributed at Patras. Anyone wishing descriptions of the IHW or copies of the Newsletter should write to: 
Ray L. Newburn, Jr.

MST -1166

Jet Propulsion Laboratory

4800 Oak Grove Drive

Pasadena, CA 91109 USA
Dr. Jurgen Rahe

Director-Remeis Observatory

Sternwartstrasse 7

D-8600 Bamberg

FEDERAL REPUBLIC OF GERMANY

Other Items. Sample copies of the Lunar and Planetary Information Bulletin prepared and distributed by the Lunar and Planetary Institute, 3303 NASA Road One, Houston, TX 77058, USA were sent to all Commission 15 members before the General Assembly. It was decided at Patras that anyone wishing to continue receiving them should inform the Bulletin editor at LPI, Frances B. Waranius, phone (713) 486-2135.

Dr. D. Morrison described the composition and activities of NASA's Planetary Exploration Committee. One very relevent action was their support for modifying the mission of the International Sun-Earth Explorer satellite, ICEE-3 to convert it into a comet tail probe. This change is now in effect and the probe will intercept the tail of Comet Giacobini-Zinner in September 1985.

\section{August 1982}

The first session briefly reviewed the IHW and allowed IHW Discipline Specialists and interested observers in each area to meet.

Session 2 was a Joint Meeting of Commissions 15 and 34 "Spectroscopic Investigations of Grains". The program consisted of three invited papers by $R$. Knacke, W. Kratschmer and B. Donn and contributed papers by V. Pirronello and L. d'Hendecourt. These are summarized in the Report of Commission 34.

\section{August 1982}

\section{Comet Halley Apparition, 1986}

J. Rahe - The International Halley Watch

D. Yeoman - Tied together orbits from 1911 back to 241 B.C. and integrated motion back to 1404 B.C. There is no evidence for significant change in brightness. Uniformity and characteristics of non-gravitational forces indicate direct rotation, no spin axis precession and no significant change in outgassing rate over this interval.

R. Newburn devised a Halley coma model from gas production/luminosity ratios of recent comets and gas dust ratio of A'Hearn.

B. McIntosh described a new model for the Halley meteor stream. He concludes meteors not perturbed since $\sim$ 1400. They lie in a shell $0.44 \mathrm{AU}$ wide and $0.04 \mathrm{AU}$ thick.

H. Fechtig described the ESA Giotto mission to Halley. Launch will be July 7 , 1985 and intercept, past perihelion will be March 13, 1986. A camera will obtain 140 images of the nucleus and a impact mass-spectrometer dust analyzer will seek the dust composition. Other experiments were not described.

Bertaux reported on the French-Soviet Vega mission with two spacecraft. After dropping probes into the Venusian atmosphere the main $\mathrm{S} / \mathrm{C}$ continued on to intercept Halley at $10^{4} \mathrm{~km}$. The first intercept will be March 8, 1986, the second a few days later. Experiments include a camera, IR and UV spectrometers, plasma wave detectors, magnetometer, and dust detector. 
Oda described the Japanese Planet A Mission. Two S/C will be launched in January and August 1985, and intercept at $0.1 \mathrm{AU}$. Experiments consist of a CCD Ly-alpha imaging device, solar wind and plasma detectors.

J. Brandt described the U.S. Space Telescope capabilities for observing Halley. Launch is planned for the first quarter of 1985. There are also plans for the Space Lab Optical Science mission to observe Halley.

E. Gerard reported a search for Conet Halley on December 2-3, 1981 with the French-Canadian telescope in Hawaii to a limiting mag of 25.2 .

Norris briefly presented a proposal for an interactive experiment on Comet Halley using an $\mathrm{OH}$ maser beam to turn off the cometary $\mathrm{OH}$ emission and watch its decay.

Dunham reported on the ISEE-3 mission to Giacobinni-Zinner.

The second Commission 15 session was on "Coma Models and Physical Processes". Invited papers by E. Barker, D. A. Mendis and M. Wallis appear in "Highlights of Astronomy".

D. Malaise gave an invited paper "The Nuclear region of Comets". Other papers were: 2. Sekanina - Two Dust Populations in Cometary Coma. V. Vanysek - Solar Corona After Impact of Coemt 1979XI; Rickman - Thermal Model of Comet Halley Nucleus; K. Jockers - In Production in the Coma via Solar Wind Charge Exchange.

\section{August 1982}

Minor Planets - Physical Properties and Future Research Plans

The four scheduled minor planet sessions were combined into two on August 24. The following papers were presented:

E. Helin - Apollo 1982DB: The Earth's Most Accessible Neighbor

E. Tedesco: Results from Recent 8-Color JHK and Radiometric Observations of Asteroids

E. Bowell and K. Lumme: A Possible New Magnitude System for Atmosphereless Solar System Objects.

A. Harris: Statistics of Asteroid Rotation Rates.

D. Watson: Physical Properties of Asteroids from IRAS.

A. Brahic: Asterex and European Plans for an Asteroid Mission.

A. Harris: Recent Asteroid Mission Planning Efforts in the United States.

\section{August 1982}

Condensation, Accretion and Early Parent Body Processes

G. Morfill - Some Cosmochemical Consequences of a Turbulent Protoplanetary Cloud.

H. J. Volk - Grain Growth in Turbulent Protostellar Accetion Disks.

B. Donn - Experiments on the Formation, Properties and Evolution of Primitive 
Grains.

J. T. Wasson - Origin of Meteoritic Chondrules and Mechanical Fractionation During the Accretion Process.

G. Wetherill - Time Scale of Planet and Planetesimal Formation.

P. Pellas - Early Thermal History of Meteorites.

L. L. Wilkening - Possible Chemical Relationships Between Comets and Asteroids Based on Their Starting Compositions.

With turbulence, the time scale for disk formation and evolution are 10 and $10^{6}$ years, respectively. Grains in disk vaporize in inner zones, vapor flows out and recondenses. This leads to element separation with distance from center. Grains limited to fraction of $\mathrm{mm}$. Solid bodies form by particle accretion but large bodies not possible unless turbulence varies in intensity. Experiments indicate amorphous, non-stoichiometric refractory grains form and current nucleation theories are not applicable. Composition of chondrites suggests everything vaporized where they formed by high temperature equilibrium condensation. There appears to be a conflict between condensation experiments and interpretation of chondrite formation conclusions. Chondrules seem to require several distinct precursors. Occurrence of $\mathrm{cm}$ size isotopic anomalous grains requires some massive particle infall from interstellar medium. Numberical treatment of accretion of planets after decay of turbulence would require $10^{5}-10^{7}$ years. If Jupiter stopped asteroid growth, it must have formed more rapidly. Formation of giant gaseous protoplanets or planetesimal formation in turbulent nebula may provide rapid formation but the answer is not clear. Comets and asteroids are smallest, therefore least changed in composition. C1 meteorites show best correlation with solar abundances but show evidence of aqueous processing. C3 may be most primitive. Low and high temperature material need to be mixed. Turbulence will do this. 
COMMISSION 16: PHYSICAL STUDY OF PLANETS AND SATELLITES (ETUDE PHYSIQUE DES PLANETES ET DES SATELLITES)

Report of Meetings 18, 19, 20, 24 August 1982

PRESIDENT: Bradford A. Smith

\section{August 1982}

COMMISSION BUSINESS

Presiding: Bradford A. Smith, President of Commission 16

Recording: Garry E. Hunt, Executive Secretary of Commission 16

\section{Election of Officers for 1982-1985}

The following slate was presented and elected unanimously by the members present:

President: Victor G. Tejfel'

Vice President: Garry E. Hunt

Executive Secretary: Andre Brahic

Organizing Committee: R.F. Beebe, J-L Berteaux, J. Elliot, D. Gautier,

L.V. Ksanfumaliti, M.Ya. Marov, H. Masursky, G. Neukum, T. Owen, V.V.

Shevshenko, B.A. Smith

2. New Members of the Commission

The following list of new members of the Commission was unanimously endorsed by the members present:
R.F. Beebe
J.A. Burns
J.J. Caldwell
J. Degewij
L.W. Esposito
P. Farinella
K. Fox
G.P. Horedt
R.F. Jurgens
A. L. Lane
J.B. Lewis
G. Neukum
M.Ya. Marov
Y. Nakagawa
L.A. Soderblom
K. Pauy
E.L. Ruskol
R.J. Terrile
E.C. Stone
D.F. Strobel.
C. F. Yoder
T.W. Thompson
L.H. Wasserman

\section{Meetings of Interest to Commission Members}

Attention was called to the following meetings that will be held prior to the next General Assembly:

A. Planetary Spectroscopy

Org: Dr. R.W. Saunders

Rutherford Appleton Laboratory

Chilton, DIDCOT

Oxon, OXII OQX, UK
Place: Oxfordshire, England

Time: 5-9 April 1983 
B. Natural Satellites

Org: Dr. Joseph A. Burns

Place: Ithaca, New York

Corne1l University

Thurston Hall

Time: 5-9 July 1983

Ithaca, NY 14853, USA

C. IUGG (including Syemour Hess

Memorial Symposium)

Place: Hamburg, FRG

Time: 15-22 August 1983

D. COSPAR

Org: Mr. Harold Masursky

U.S. Geological Survey

2255 N. Gemini Drive

Flagstaff, Arizona 85701, USA

Place: Graz, Austria

Time: 25 June-7 Ju1y 1984

This is not intended to be a complete list. Members who wish to bring additional meetings to the attention of the Commission 16 membership should contact the incoming Executive Secretary, Dr. A. Brahic.

\section{COSPAR Decisions of interest to Commission 16 Membership}

a) COSPAR has set up a group concerned with a reference atmosphere for Venus. Members are Klione, Moroz, Bauer, Biswas, Hunten, Marov, Seiff and Taylor.

b) COSPAR has issued a statement to the national space agencies urging them to try to find sufficient funds to ensure that more regular space missions are carried out in planetary exploration. COSPAR is deeply concerned by the reduction in solar sytem space missions, as has occured during the last decade.

\section{Planetary Nomenclature}

H. Masursky reported that meetings of the IAU Working Group for Planetary System Nomenclature have been held on 14 and 15 August in Patras to discuss outstanding issues relating to nomenclature of solar system objects. Unresolved issues included the naming of newly discovered satellites and the question of how to treat the numerous rings and gaps in the Saturn ring system.

\section{Other Commission Business}

There was a brief discussion of problems with archival storage of digital data. B. Smith expressed concern over the current status of large amounts of digital data from planetary spacecraft which, through poor archiving and loss of related documentation, are losing some of their value. He indicated that Mariner 9 and Viking data were good examples of large data sets that could be lost to future investigators. The problem has become more acute as increasing numbers of research groups have access to digital image processing systems and prefer to work with the original digital data rather than hard copy photographs. Smith noted that some Mariner 9 and Viking data were already irretrievably 10 st. Following discussion of this matter, the members present suggested that a small group draft a proposal for subsequent discussion as a possible means of getting an IAU resolution supporting improved archiving and documentation procedures for digital data at appropriate national data storage facilities. The President appointed G. Hunt, T. Johnson and H. Masursky to prepare a draft proposal.

Note: Because of inadequate time to prepare this proposal, the action has been deferred until the next General Assembly. 


\title{
I. SCIENTIFIC SESSION
}

Chairman: B.A. Smith

\author{
S.K. Runcorn \\ F. Meyer \\ E. A. Whitaker \\ P. Moore \\ R.H. Brown et al. \\ C.F. Capen
}

G.P. Mantas

I.P. Williams

M.A. Janssen et a1.

R. Beer and M.A. Janseen

B. Petropoulos et al.

G.E. Hunt

I. de Pater

Y. Leblanc

A. Dollfus

T.V. Johonson

M.Ya. Marov

\author{
Lunar Magnetism and Its Implications \\ Creation of a Data File for Lunar Profiles \\ from a New Set of Measurements \\ The Lunar Procellarum Basin \\ Recent Results of Lunar Transient \\ Phenomena \\ Diameters and Albedos of the Satellites of \\ Uranus and Neptune \\ International Mars Observation Computer \\ Program Studies of Seasonal Meteorological \\ Phenomena and Current Changes in the Martian \\ Arctic \\ Comparison of Theoretical and Observed \\ Photoelectron Energy Spectra in the \\ Ionosphere of Mars \\ The Breakup of Comets to Form Meteor Streams \\ High Resolution Microwave Imaging of the \\ Venus Atmosphere \\ High Resolution Spectroscopy and Narrow-Band Imaging \\ of Venus Around $4.8 \mathrm{~m}$ \\ A Model for the Upper Atmosphere of Jupiter after \\ Voyager's Measurements \\ Voyager Observations of Vortex Streets in the \\ Atmosphere of Saturn \\ New High Resolution Radio Observation of Jupiter \\ and Saturn \\ Jovian and Saturnian Radio Emissions Observed with \\ the Voyager Spacecraft Radio Experiment \\ First Planetary Results from the 2-m Telescope at \\ Plc-du-Midi \\ Galileo Mission to Jupiter \\ Future Soviet Planetary Missions
}

\section{August 1982}

I. JOINT DISCUSSION WITH COMMISSION 44: EXPLORATION OF THE SOLAR SYSTEM Organizing Committee: R. Von Eshelman, D.M. Hunt, H. Masursky, T.C. Owen, G.H. Pettengill, B.A. Smith, G.L. Tyler (USA); G.E. Hunt (Chairman), J.E. Guest (UK); M.Ya. Marov (USSR); J.E. Blamont, A. Brahic (France)

A. VOYAGER ENCOUNTER WITH SATURN

Chairman: G.E. Hunt

1. Magnetosphere of Saturn

F. Scarf

2. Satellites of Saturn

3. Rings of Saturn

4. Structure and Composition of the Atmosphere of Saturn

D. Morrison

5. Meteorology of Saturn

J. Cuzzi

T. Owen

R. Beebe

6. Titan

$\mathrm{T}$. Owen 
B. PIONEER VENUS AND VENERA STUDIES OF VENUS

\section{Chairman: B.A. Smith}

1430-1500

$1500-1530$

$1530-1600$

Surface of Venus

Chemistry and Clouds of Venus

Atmospheric Composition and

Evolution of Venus Atmosphere

$1630-1700$

$1700-1720$

$1720-1740$

1740-1800

Interaction of Venus and Solar
Wind

Ground Based Radar Mapping

Pioneer Venus Radar Mapping

The Venus Nature: A Discussion on New Findings
H. Masursky

R. Prinn

T. Donahue

I. Brace

D. Campbe11

G. Petteng 111

M. Marov

\section{August 1982}

WORKING GROUP ON CARTOGRAPHIC COORDINATES

Presiding: M.E. Davies, Chairman

A meeting of the Working Group on Cartographic Coordinates and Rotational Elements of the Planets and Satellites was held with members of both Commissions 4 and 16 in attendance. The report of the Working Group is included in this report of Commission 16. 
REPORT OF THE IAU WORKING GROUP ON CARTOGRAPHIC COORDINATES AND ROTATIONAL ELEMENTS OF THE PLANETS AND SATELLITES: 1982

CHAIRMAN: M.E. Davies

MEMBERS: V.K. Abalakin, J.H. Lieske, P.K. Seidelmann,

A.T. Sinclair, A.M. Sinzi, B.A. Smith

CONSULTANT: Y.S. Tjuflin

\section{Introduction}

The IAU Working Group on Cartographic Coordinates and rotational Elements of the Planets and Satellites was established as a consequence of resolutions adopted by Commisssions 4 and 16 at the IAU General Assembly at Grenoble in 1976. The first report of the Working Group was presented to the General Assembly at Montreal in 1979 and published in the Trons. IAU, pp.72-79, 1980. The report with appendices was published in Celestial Mechoon ies, 22, pp. 205-230, 1980. The guiding principles and conventions that were adopted by the Group and the rationale for their acceptance are presented in that report and its appendixes and will not be reviewed here.

This report will incorporate changes in the tables giving the directions of the north poles of rotation and the prime meridians of the planets and satellites expressed in standard equatorial coordinates of 1950.0. New tables give these same parameters with respect to the standard equator and equinox of $J 2000$. The tables giving the sizes and shapes of the planets and satellites have also been revised.

\section{Definition of Rotational Elements}

The north pole is that pole of rotations which lies on the north side of the invariable plane of the solar system. At this time, two standard coordinate systems are in common use; they are designated B1950 and J2000. B1950 is defined with respect to the fundamental FK4 catalog and J2000 is defined with respect to the new FK5 catalog. B1950 has the standard epoch (J1950) of 1950 January 1.0, ET(JED 2433282.5) and J2000 has the standard epoch (J2000) of 2000 January 1.5 (JD 2451545.0), TDB. The variable quantities are expressed in units of days (86400 SI seconds) or Jullan centuries of 36525 days.

The direction of the north pole is specified by the value of its right ascension $\alpha$ and declination $\delta$; whereas the orientation of the prime meridian is specified by the angle $"$ ! that is measured along the planet's equator in the positive sense with respect to the planet's north pole (i.e., in an easterly direction on the planet's surface) from the ascending node of the planet's equator on the standard equator to the point where the prime meridian crosses the planet's equator. Because the prime meridian is assumed to rotate uniformly with the planet, $W$ accordingly varies linearly with time. In addition, $\alpha, \delta$, and $W$ may vary with time due to a precession of the axis of rotation of the planet (or satellite). If $"$ increases with time, the planet has a direct (or prograde) rotation relative to the invariable plane; if $W$ decreases with time, the rotation is said to be retrograde.

In the absence of other information, the axis of rotation is assumed to be normal to the mean orbital plane; Mercury and most of the satellites are in this category. For many of the satellites, it is assumed that the rotation rate is equal to the mean orbital period.

The angle $W$ speciftes the ephemeris position of the prime meridian, and for the planets or satellites without any accurately observable fixed surface 
features the adopted expression for $W$ defines the prime meridian and is not subject to correction. Where possible, however, the cartographic positions of the prime meridian is defined by a suitable observable feature and so the constants in the expression $W=W+W d$, where $d$ is the interval in days from the standard epoch, are chosen so that the ephemeris position follows the motion of the cartographic position as closely as possible; in these cases the expression for $W$ may require emendation in the future.

Recommended values of the constants in the expression for $\alpha_{0}, \delta_{0}$, and $W$ are given for the planets and satellites in Tables 1 and 2 for the standard equatorial coordinates with equinox B1950 at epoch $\mathrm{J} 1950$ and for the planets and satellites in Tables 3 and 4 for the standard equotorial coordinates with equinox $\mathrm{J} 2000$ at epoch $\mathrm{J} 2000$. In general these expressions should be accurate to one-tenth of a degree; however, two decimal places are given to assure consistency when changing coordinate systems. Zeros are added to rate values (W) for computational consistency and are not an indication of significant accuracy. Three decimal places are given in the expresstons for the Moon and Mars, reflecting the greater confidence in their accuracy. Expressions for the Sun and Earth, are given to a similar precision as those of the other bodies of the solar system for comparative purposes only.

\section{Definition of Cartographic Coordinates Systems}

Both planetocentric and planetographic systems of coordinates are used in the study of the planets and satellites. Both systems are based on the same fundamental reference axis but differ, as explained below, in the definitions of latitude and longitude. Planetocentric coordinates are used for general purposes and are based on a right-handed system of axes, whereas planetographic coordinates are used for cartographic purposes and depend on the adoption of additional parameters to define a reference surface, usually a spheroid, that approximates an equipotential surface of the planet.

For both systems, the fundamental reference z-axts is the mean axis of rotation and the planetary equator is the plane that is normal to this axis and passes through the center of mass of the planet. The $x$-axis is defined by the intersection of the equatorial plane with the plane of the prime meridian, whose position is defined in an arbitrary manner. The $y$-axis of planetocentric rectangular coordinates is defined so as to form a right-handed system.

Latitude is measured north and south of the equator; north latitudes are designed as positive. The planetocentric latitude ( $\phi)$ of a point is the angle between the equatorial plane and the line connecting the point to the center of mass. The planetographic latitude $\left(\phi^{\prime}\right)$ of a point on the reference surface is the angle between the equatorial plane and the normal to the reference surface at the point.

Longitude is measured around the equatorial plane from the prime meridian from $0^{\circ}$ to $360^{\circ}$. Planetocentric longitudes $(\lambda)$ are measured positively to the east, whereas planetographic longitudes $\left(\lambda^{\prime}\right)$ are measured in the direction opposite to the rotation, i.e., positively to the west in the case of direct rotation. Planetocentric longitudes are measured from the ephemeris position of the prime meridian as defined by the rotational elements, whereas planetographic longitudes are measured from the cartographic position to the prime meridian as defined by the adopted longitude of some clearly observable surface feature. These two positions may normally be assumed to coincide, but it is conceivable that errors in the rotational elements may cause the cartographic position to drift away from the ephemeris position by a small amount $\Delta W$, where $\Delta W$ is measured positively to the east of the ephemeris position. 
Planetcentric rad $u s(R)$ is measured from the center of mass to the point concerned. In the planetographic system the position of a point ( $P$ ) not on the reference surface is specified by the planetographic longitude and latitude of the point $\left(P^{\prime}\right)$ on the reference surface at which the normal passes through $P$ and by the height (h) of $P$ above $P^{\prime}$.

The reference surfaces for most of the planets are spheroids for which the radius of the equator $(A)$ is larger than the polar semiaxis (C). For some planets and most satellites the reference surface is a sphere $(A=C)$, and the planetocentric and planetographic latitutes are then numerically the same. The polar axis of each reference surface is assumed to be the mean axis of rotation as defined by the adopted rotational elements since the accuracy of measurement cannot, at present, observe a motion of the axis of rotation with respect to the axis of figure.

The recommended values of the parameters for the reference surfaces for planets and satellites are given in Table 5. Radii for irregular-shaped satellites are given in Table 6.

It should be noted that east longitude on the Sun, Earth, and Moon is commonly considered to be in the positive direction and that longitudes are usually measured $0^{\circ}$ to $180^{\circ}$ east and west. 
Table 1. Recommended Values for the Direction of the North Pole of Rotation and the Prime Meridian of the Sun and Planets (1982).
$\alpha_{0}, \delta_{0}$ are standard equatorial coordinates with equinox B1950 at epoch J1950. Approximate coordinates of the north pole of the invariable plane are $\alpha=272.40, \delta=+66^{\circ} .99$.
$\mathrm{T}=$ interval in Julian ephemeris centuries (of 36525 days) from the standard epoch.
$\mathrm{d}=$ interval in ephemeris days from the standard epoch.
The standard epoch is 1950 January $1.0 \mathrm{ET}$, i.e., JED 2433282.5

\begin{tabular}{|c|c|c|c|c|c|}
\hline Sun & $\begin{array}{l}\alpha \\
\delta^{\circ} \\
w^{\circ}\end{array}$ & $\begin{array}{r}=285.90 \\
=63.90 \\
=240.90\end{array}$ & + & $14^{\circ} .1844000 \mathrm{~d}$ & \\
\hline Mercury & $\begin{array}{l}\alpha \\
\delta^{\circ} \\
\omega^{\circ}\end{array}$ & $\begin{array}{l}=280.90 \\
=61.40 \\
=184.74\end{array}$ & $\begin{array}{l}- \\
+\end{array}$ & $\begin{array}{l}0.033 \mathrm{~T} \\
0.005 \mathrm{~T} \\
6.1385025 \mathrm{~d}\end{array}$ & (a) \\
\hline Venus & $\begin{array}{l}\alpha \\
\delta_{0}^{\circ} \\
\omega\end{array}$ & $\begin{array}{l}=272.80 \\
=\quad 67.20 \\
=213.63\end{array}$ & - & $1.4814205 \mathrm{~d}$ & \\
\hline Earth & $\begin{array}{l}a_{0}^{0} \\
\delta_{0}^{0}\end{array}$ & $\begin{array}{r}=0.00 \\
=\quad 90.00 \\
99.87\end{array}$ & $\begin{array}{l}- \\
+\end{array}$ & $\begin{array}{c}0.640 \mathrm{~T} \\
0.557 \mathrm{~T} \\
360.9856123 \mathrm{~d}\end{array}$ & (b) \\
\hline Mars & $\begin{array}{l}\alpha \\
\delta_{0}^{\circ} \\
W^{0}\end{array}$ & $\begin{array}{l}=317.342 \\
=\quad 52.711 \\
=\quad 11.504\end{array}$ & $\begin{array}{l}\overline{-} \\
+\end{array}$ & $\begin{array}{c}0.108 \mathrm{~T} \\
0.061 \mathrm{~T} \\
350.8919830 \mathrm{~d}\end{array}$ & (c) \\
\hline Jupiter & $\begin{array}{l}\alpha \\
\delta^{\circ} \\
h^{\circ}\end{array}$ & $\begin{array}{l}=268.00 \\
=\quad 64.50 \\
=\quad 80.60\end{array}$ & $\begin{array}{l}- \\
+ \\
+\end{array}$ & $\begin{array}{c}0.008 \mathrm{~T} \\
0.003 \mathrm{~T} \\
870.5360000 \mathrm{~d}\end{array}$ & (d) \\
\hline Saturn & $\begin{array}{c}a \\
\delta_{0}^{\circ} \\
w\end{array}$ & $\begin{array}{l}=38.50 \\
=83.31 \\
=\quad 76.81\end{array}$ & - & $\begin{array}{c}0.034 \mathrm{~T} \\
0.004 \mathrm{~T} \\
810.7939024 \mathrm{~d}\end{array}$ & (d) \\
\hline Uranus & $\begin{array}{l}\alpha \\
\delta^{\circ} \\
\omega^{\circ}\end{array}$ & $\begin{array}{l}=256.72 \\
=-15.04 \\
=360.00\end{array}$ & - & $554.9130000 \mathrm{~d}$ & \\
\hline Neptune & $\begin{array}{l}\alpha \\
\delta_{0}^{\circ} \\
\omega^{0}\end{array}$ & $\begin{array}{l}=294.91 \\
=\quad 40.53 \\
=\quad 360.00\end{array}$ & + & $468.7500000 \mathrm{~d}$ & \\
\hline Pluto & $\begin{array}{l}\alpha \\
\delta_{0}^{0} \\
w^{0}\end{array}$ & $\begin{array}{r}=311.00 \\
=4.00 \\
=360.00\end{array}$ & - & $56.3640000 \mathrm{~d}$ & \\
\hline
\end{tabular}

Note (a) The $20^{\circ}$ meridian is defined by the crater Hun Kal.

(b) The $0^{o}$ meridian is defined by the transit circle at Greenwich, England.

(c) The $0^{\circ}$ meridian is defined by the crater Airy-0.

(d) The equations for $W$ for Jupiter and Saturn refer to the rotation of their magnetic fields (System III). On Jupiter, System I $\left(\mathrm{W}_{\mathrm{T}}=17.7+877^{\circ} .900 \mathrm{~d}\right)$ refers to the mean atmospheric equatorial rotation; System II $\left(W_{I I}=16^{\circ} .8+870.270 \mathrm{~d}\right)$ refers to the mean 
atmospheric rotation north of the south component of the north equatorial belt, and south of the north component of the south equatorial belt.

Table 2. Recommended Values for the Direction of the North Pole of Rotation and the Prime Meridian of the Satelittes (1982).

$\alpha_{0}, \delta_{0}, \mathrm{~T}$, and $\mathrm{d}$ have the same meanings as Table 1 (epoch 1950 January 1.0 ET, i.e., JED 2433282.5)

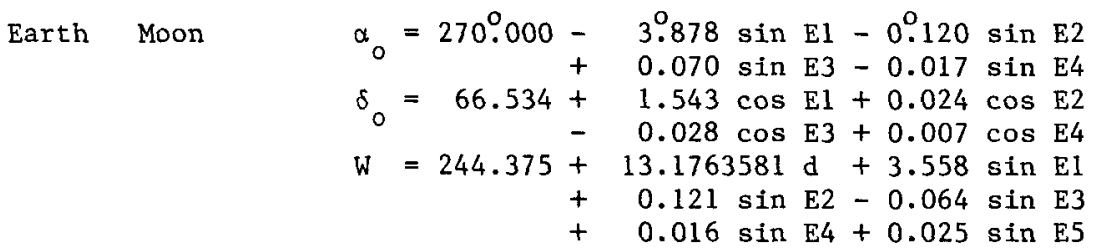

where $\mathrm{El}=12.112-0.052992 \mathrm{~d}, \mathrm{E} 2=24^{\circ} .224-0^{0} .105984 \mathrm{~d}$

$\mathrm{E} 3=227.645-13.012000 \mathrm{~d}, \mathrm{E} 4=261.105+13.340716 \mathrm{~d}$

$\mathrm{E} 5=358.000-0.985600 \mathrm{~d}$,

$$
\begin{aligned}
& \text { Mars Phobos } \alpha_{0}=317^{\circ} .31-0.108 \mathrm{~T}+1^{\circ} .79 \sin \mathrm{M}_{1} \\
& \begin{array}{l}
\delta^{0}=52.70-0.061 \mathrm{~T} \\
\mathrm{~W}^{0}=270.23+1128.8444790 \mathrm{~d}+0.66 \times 10^{-8} \mathrm{~d}^{2}
\end{array} \\
& -1.42 \sin M_{1}-2.31 \sin M_{2} \\
& \text { Deimos } \alpha_{0}=316.29-0.108 \mathrm{~T} \quad+2.98 \sin \mathrm{M}_{1} \\
& \begin{array}{l}
\delta^{0}=53.33-0.061 \mathrm{~T} \\
\mathrm{~W}^{0}=69.97+285.1619030 \mathrm{~d}-0.4 \times 10^{-9} \mathrm{~d}^{2}-2.66 \sin \mathrm{M}_{1}
\end{array}
\end{aligned}
$$

where $M_{1}=206^{\circ} .34-0.435764 \mathrm{~d} \mathrm{M}_{2} 88^{\circ} .80+1128.409670 \mathrm{~d}+0.66 \times 10^{-8} \mathrm{~d}^{2}$

Jupiter: Amalthea

$$
\begin{aligned}
& \alpha=268.00-0.008 \mathrm{~T} \\
& \delta^{0}=64.50+0.003 \mathrm{~T} \\
& W^{0}=50.20+722.6303746 \mathrm{~d}
\end{aligned}
$$

Io

$$
\begin{aligned}
& \alpha=268^{\circ} .00-0.008 \mathrm{~T}+0.094 \sin \mathrm{J} 1+0^{\circ} .024 \sin \mathrm{J} 2 \\
& \delta^{\circ}=64.50+0.003 \mathrm{~T}+0.040 \cos \mathrm{J} 1+0.011 \cos \mathrm{J} 2 \\
& W^{\circ}=262.72+203.4889538 \mathrm{~d}-0.085 \sin \mathrm{J} 1-0.022 \sin \mathrm{J} 2
\end{aligned}
$$

Europa

$$
\begin{aligned}
\alpha_{0}=268.03-0.008 \mathrm{~T} & +1.086 \sin \mathrm{J} 2+0.060 \sin \mathrm{J} 3 \\
& +0.015 \sin \mathrm{J} 4+0.009 \sin \mathrm{J} 5 \\
\delta_{0}=64.52+0.003 \mathrm{~T} & +0.468 \cos \mathrm{J} 2+0.026 \cos \mathrm{J} 3 \\
& +0.007 \cos \mathrm{J} 4+0.002 \cos \mathrm{J} 5 \\
\mathrm{~W}=158.44+101.3747235 \mathrm{~d} & -0.980 \sin \mathrm{J} 2-0.054 \sin \mathrm{J} 3(\mathrm{a}) \\
& -0.014 \sin \mathrm{J} 4-0.008 \sin \mathrm{J} 5
\end{aligned}
$$

$$
\text { Ganymede } \begin{aligned}
\alpha_{0}=268.15-0.008 \mathrm{~T} \quad & -0.037 \sin \mathrm{J} 2+0.431 \sin \mathrm{J} 3 \\
\delta_{0}=64.57+0.091 \sin \mathrm{J} 4 & +0.003 \mathrm{~T} \quad-0.016 \cos \mathrm{J} 2+0.186 \cos \mathrm{J} 3 \\
& \\
\mathrm{~W}=196.82+0.039 \cos \mathrm{J} 4 & +50.3176081 \mathrm{~d}+0.033 \sin \mathrm{J} 2-0.389 \sin \mathrm{J} 3(\mathrm{~b}) \\
& -0.082 \sin \mathrm{J} 4
\end{aligned}
$$




$$
\begin{aligned}
& \text { Callisto } \alpha_{0}=268.68-0.008 \mathrm{~T} \quad-0.068 \mathrm{sin} \mathrm{J} 3+0.590 \mathrm{sin} \mathrm{J4} \\
& \delta_{0}=64.83+0.003 \mathrm{~T} \quad-0.029 \cos \mathrm{J} 3+0.254 \cos \mathrm{J} 4 \\
& \mathrm{~W}=157.55+21.5710715 \mathrm{~d}+0.061 \sin \mathrm{J} 3-0.533 \sin \mathrm{J} 4(\mathrm{c}) \\
& -0.009 \text { sin J6 }
\end{aligned}
$$

where $\mathrm{J} 1=19^{\circ} .20+4850^{\circ} .7 \mathrm{~T}, \mathrm{~J} 2=120^{\circ} .80+1191.3 \mathrm{~T}, \mathrm{~J} 3=349^{\circ} .50+262^{\circ} .1 \mathrm{~T}$, $\mathrm{J} 4=198.30+64.3 \mathrm{~T}, \mathrm{~J} 5=241.60+2382.6 \mathrm{~T}, \mathrm{~J} 6=317.70+6070.0 \mathrm{~T}$

Saturn: Mimas

$$
\begin{aligned}
& \alpha=38.50-0.034 \mathrm{~T}+13.13 \sin \mathrm{S} 1 \\
& \delta^{\circ}=83.31-0.004 \mathrm{~T}-1.53 \cos \mathrm{S} 1 \\
& \omega^{\circ}=246.80+381.9945550 \mathrm{~d}-13.04 \sin \mathrm{S} 1-43.41 \sin \mathrm{S} 6
\end{aligned}
$$

Enceladus $\alpha_{0}=38.50-0.034 \mathrm{~T}$

$\delta^{0}=83.31-0.004 \mathrm{~T}$

$\mathrm{W}^{\mathrm{O}}=301.41+262.7318996 \mathrm{~d}$

Tethys

$$
\begin{aligned}
& \alpha=38.50-0.034 \mathrm{~T}+9.36 \sin \mathrm{S} 2 \\
& \delta^{0}=83.31-0.004 \mathrm{~T}-1.09 \cos \mathrm{S} 2 \\
& \mathrm{~W}^{0}=31.76+190.6979085 \mathrm{~d}-9.29 \sin \mathrm{S} 2+2.16 \sin \mathrm{S} 6
\end{aligned}
$$

Dione

$$
\begin{aligned}
& \alpha=38.50-0.034 \mathrm{~T} \\
& \delta^{0}=83.31-0.004 \mathrm{~T} \\
& W^{0}=121.54+131.5349316 \mathrm{~d}
\end{aligned}
$$

Rhea

$$
\begin{aligned}
& \alpha=38.22-0.034 \mathrm{~T}+3.00 \sin \mathrm{S} 3 \\
& \delta^{0}=83.34-0.004 \mathrm{~T}-0.35 \cos \mathrm{S} 3 \\
& \mathrm{~W}^{0}=14.52+79.6900478 \mathrm{~d}-2.98 \sin \mathrm{S} 3
\end{aligned}
$$

Titan $\quad \alpha^{\circ}=34.30-0.034 \mathrm{~T}+2.58 \sin \mathrm{S} 4$
$\delta^{\circ}=83.71-0.004 \mathrm{~T}-0.30 \cos \mathrm{S} 4$
$\mathrm{~W}^{\circ}=79.10+22.5769768 \mathrm{~d}-2.56 \sin \mathrm{S} 4$

$$
\begin{aligned}
\text { Hyperion } \alpha^{0}=33.42-0.034 \mathrm{~T}+4.89 \sin \mathrm{S} 5+2.75 \sin \mathrm{S} 4 \\
\delta_{0}=83.80-0.004 \mathrm{~T}-0.57 \cos \mathrm{S}-0.32 \cos \mathrm{S} 4 \\
W^{\circ}=344.75+16.9199514 \mathrm{~d}-4.86 \sin \mathrm{S} 5-2.73 \sin \mathrm{S} 4 \\
+9.09 \sin \mathrm{S} 7 \\
\text { Iapetus } \alpha_{0}=289^{\circ} .26
\end{aligned}
$$$$
\begin{aligned}
& \alpha_{0}=289.26 \\
& \delta^{0}=78.73
\end{aligned}
$$$$
\mathrm{W}^{0}=275.36+4.5379571 \mathrm{~d}
$$

where $\mathrm{S} 1=68^{\circ} .60-36505^{\circ} .5 \mathrm{~T}, \mathrm{~S} 2=314^{\circ} .50-7225^{\circ} .9 \mathrm{~T}, \mathrm{~S} 3=134^{\circ} .90-1016^{\circ} .3 \mathrm{~T}$, $\mathrm{S} 4=57.40-52.1 \mathrm{~T}, \mathrm{~S} 5=22.60-239.2 \mathrm{~T}, \mathrm{~S} 6=64.90+506.2 \mathrm{~T}$, $\mathrm{S} 7=277.40+20528.5 \mathrm{~T}$

Uranus: Miranda $\alpha=256^{\circ} .72$

$$
\begin{aligned}
\delta^{\circ} & =-15.04 \\
W^{\circ} & =59.20-254^{\circ} .5968883 \mathrm{~d}
\end{aligned}
$$

$$
\text { Arie1 } \quad \begin{aligned}
\alpha & =256.72 \\
\delta^{0} & =-15.04 \\
W^{0} & =47.30-142.8356047 \mathrm{~d}
\end{aligned}
$$

Umbriel $\alpha_{\alpha}=256.72$

$$
\begin{aligned}
& \delta^{0}=-15.04 \\
& W^{0}=146.40-86.8688136 \mathrm{~d}
\end{aligned}
$$



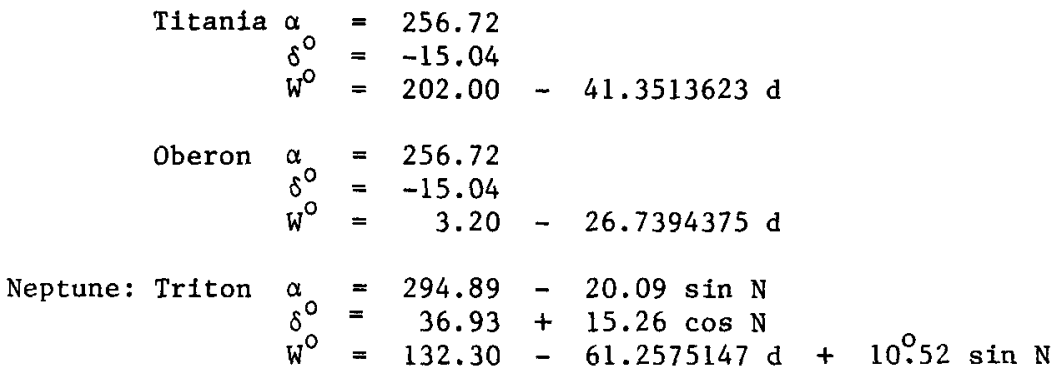

whwere $\mathrm{N}=158^{\circ} .34+62.0 \mathrm{~T}$

Pluto: Charon $\alpha=311^{\circ} .00$

$$
\begin{aligned}
\alpha & =311.00 \\
\delta^{0} & =4.00 \\
W^{0} & =133.00-56.3640000 \mathrm{~d}
\end{aligned}
$$
Note (a) The $180^{\circ}$ mexidian is defined by the crater Cilix.
(b) The 1280 meridian is defined by the crater Anat.
(c) The $326^{\circ}$ meridian is defined by the crater Saga.
(d) The $162^{\circ}$ meridian is defined by the crater Palomides.
(e) The $5^{\circ}$ meridian is defined by the crater Salih.
(f) The $299^{\circ}$ meridian is defined by the crater Arete.
(g) The $63^{\circ}$ meridian is defined by the crater Palinurus.
(h) The $340^{\circ}$ meridian is defined by the crater Tore.
(i) The $196^{\circ}$ meridian is defined by the crater Bahloo.
(j) The $276^{\circ}$ meridian is defined by the crater Almeric.

Table 3. Recommended Values for the Direction of the North Pole of Rotation and the Prime Meridian of the Sun and Planets (1982).

\footnotetext{
$\alpha_{0}, \delta_{0}$ are standard equatorial coordinates with equinox J2000 at epoch J2000. Approximate coordinates of the north pole of the invariable plane are $\alpha=272.38, \delta=+66.99$.

$T=$ interval in Julian centuries (of 36525 days) from the standard epoch.

$\mathrm{d}=$ interval in days from the standard epoch.
}

\begin{tabular}{|c|c|c|c|c|c|}
\hline Sun & $\begin{array}{l}\alpha \\
\delta^{0} \\
W^{0}\end{array}$ & $\begin{array}{l}= \\
= \\
=\end{array}$ & $\begin{array}{r}285^{\circ} .96 \\
63.96 \\
84.11\end{array}$ & + & $14^{\circ} .1844000 \mathrm{~d}$ \\
\hline Mercury & $\begin{array}{l}\alpha \\
\delta^{0} \\
W^{o}\end{array}$ & $\begin{array}{l}= \\
= \\
=\end{array}$ & $\begin{array}{r}281.02 \\
61.45 \\
329.71\end{array}$ & $\begin{array}{l}- \\
- \\
+\end{array}$ & $\begin{array}{l}0.033 \mathrm{~T} \\
0.005 \mathrm{~T} \\
6.1385025 \mathrm{~d}\end{array}$ \\
\hline Venus & $\begin{array}{l}\alpha \\
\delta^{0}\end{array}$ & $\begin{array}{l}= \\
= \\
=\end{array}$ & $\begin{array}{r}272.78 \\
61.21 \\
159.91\end{array}$ & - & $1.4814205 \mathrm{~d}$ \\
\hline Earth & $\begin{array}{l}\alpha \\
\delta^{0} \\
w^{o}\end{array}$ & $\begin{array}{l}= \\
= \\
=\end{array}$ & $\begin{array}{r}0.00 \\
90.00 \\
100.21\end{array}$ & $\begin{array}{l}- \\
+\end{array}$ & $\begin{array}{c}0.641 \mathrm{~T} \\
0.557 \mathrm{~T} \\
360.9856123 \mathrm{~d}\end{array}$ \\
\hline
\end{tabular}

The standard epoch is 2000 January 1.5 , i.e., JD 2451545.0 TDB. 


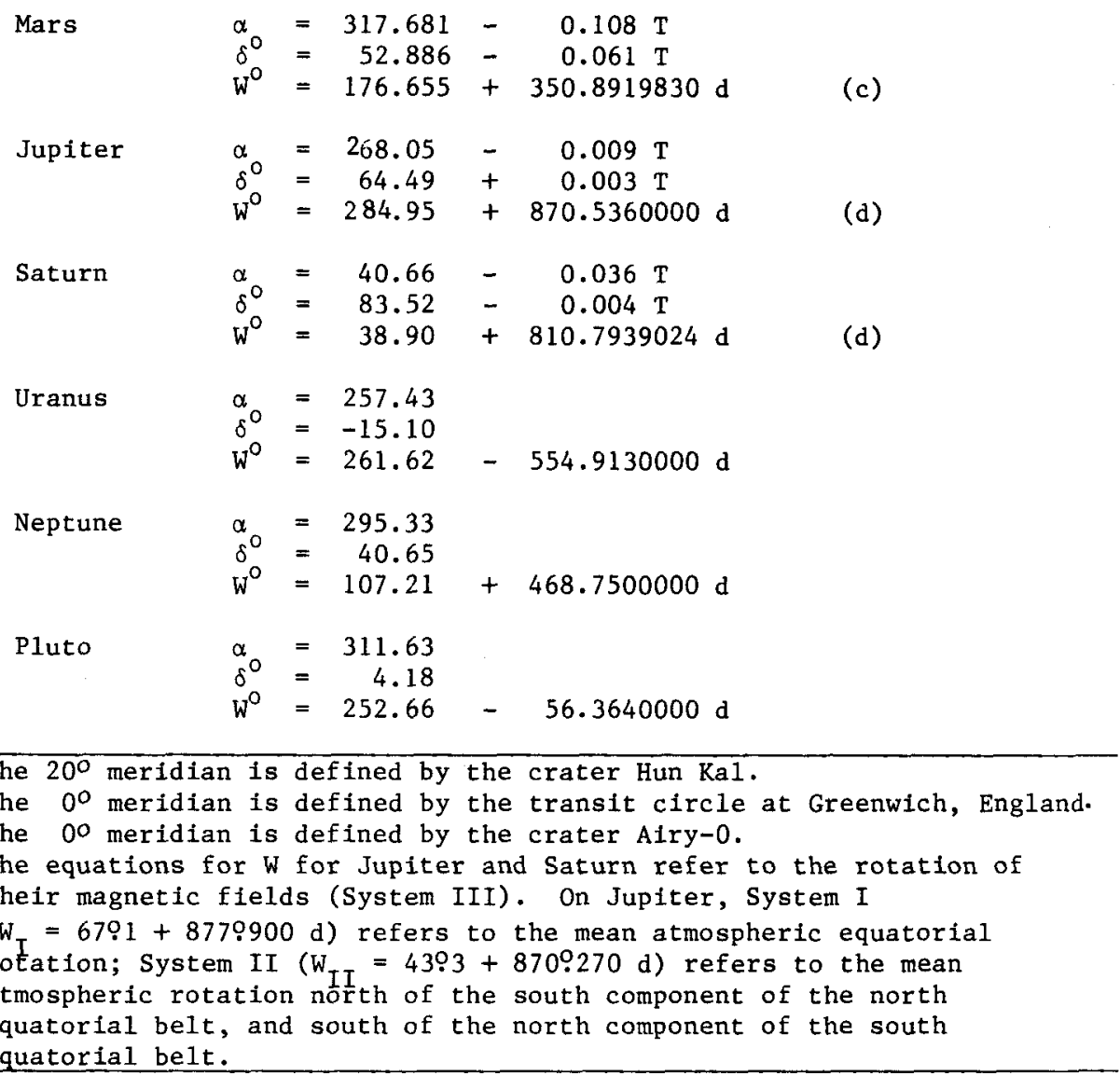

Table 4. Recommendation Values for the Direction of the North Pole of Rotation and the Prime Meridian of the Satellites (1982).

$\alpha, \delta, T$, and $d$ have the same meanings as in Table 3 (epoch 2000 January 1.5 , i.e., JD 2451545.0 TDB)

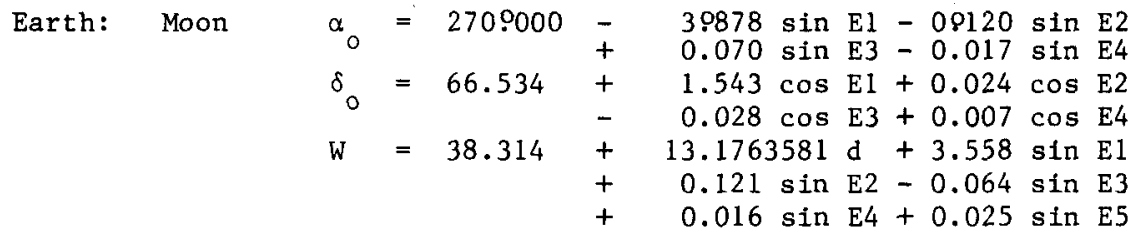

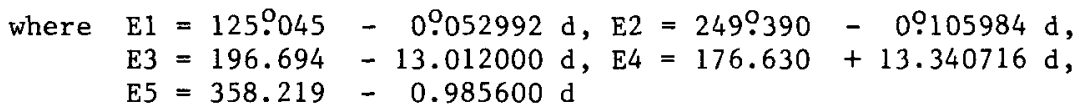


Table 4. Recommended Values for the Direction of the North Pole of Rotation Mars: Phobos $\begin{aligned} \alpha_{0} & =317.65-0.108 \mathrm{~T}+1.80 \sin \mathrm{M}_{1} \\ \delta_{0} & =52.87-0.061 \mathrm{~T}-1.08 \cos \mathrm{M}_{1} \\ & =32.84+1128.8444790 \mathrm{~d}+0.66 \times 10^{-8} \mathrm{~d}_{\mathrm{d}} \\ & -1.43 \sin \mathrm{M}_{1}-2.32 \sin \mathrm{M}_{2} \\ & =316.62-0.108 \mathrm{~T}+3.00 \sin \mathrm{M}_{1} \\ \text { Deimos } & =53.50-0.061 \mathrm{~T}-1.78 \cos \mathrm{M}_{1} \\ & =79.55+285.1619030 \mathrm{~d}-0.4 \times 10^{-91} \mathrm{~d}^{2}-2.68 \sin \mathrm{M}_{1}\end{aligned}$ where $M_{1}=169951-0.435764 \mathrm{~d} \mathrm{M}_{2}=190971+11289409670 \mathrm{~d}+0.66 \times 10^{-8} \mathrm{~d}^{2}$

$$
\begin{aligned}
& \text { Jupiter: Amalthea }=268.05-0.009 \mathrm{~T} \\
& =64.49+0.003 \mathrm{~T} \\
& =208.06+72296303746 \mathrm{~d} \\
& \text { Io } \quad=268.05-0.009 \mathrm{~T}+0.094 \sin \mathrm{J} 1+0.024 \sin \mathrm{J} 2 \\
& =64.50+0.003 \mathrm{~T}+0.040 \cos \mathrm{J} 1+0.011 \cos \mathrm{J} 2 \\
& =200.39+203.4389538 \mathrm{~d}-0.085 \sin \mathrm{J} 1-0.022 \sin \mathrm{J} 2 \\
& \text { Europa }=268.08-0.009 \mathrm{~T}+1.086 \sin \mathrm{J} 2+0.060 \sin \mathrm{J} 3 \\
& +0.015 \sin \mathrm{J} 4+0.009 \sin \mathrm{J} 5 \\
& =64.51+0.003 \mathrm{~T}+0.468 \cos \mathrm{J} 2+0.026 \cos \mathrm{J} 3 \\
& +0.007 \cos \mathrm{J} 4+0.002 \cos \mathrm{J} 5 \\
& =34.97+101.3747235 \mathrm{~d}-0.980 \sin \mathrm{J} 2-0.054 \sin \mathrm{J} 3 \\
& -0.014 \sin \mathrm{J} 4-0.008 \sin \mathrm{J} 5 \\
& \text { Ganymede }=268.20-0.009 \mathrm{~T} \quad-0.037 \sin \mathrm{J} 2+0.431 \sin \mathrm{J} 3
\end{aligned}
$$

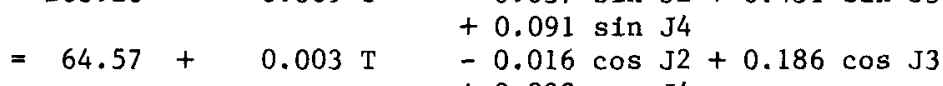

$$
\begin{aligned}
& =42.79+50.3176081 \mathrm{~d}+0.033 \sin \mathrm{J} 2-0.389 \sin \mathrm{J} 3 \\
& -0.082 \sin \mathrm{J} 4 \\
& \text { Callisto }=268.72-0.009 \mathrm{~T}-0.068 \sin \mathrm{J} 3+0.590 \sin \mathrm{J} 4 \\
& \begin{aligned}
& +0.010 \sin \mathrm{J} 6 \\
& -0.029 \cos \mathrm{J} 3+0.254 \cos \mathrm{J} 4
\end{aligned} \\
& =259.90+21.5710715 \mathrm{~d}+0.0061 \cos \mathrm{J} 6 \\
& -0.009 \sin J 6
\end{aligned}
$$

where $\mathrm{J} 1=283^{\circ} .90+4850^{\circ} .7 \mathrm{~T}, \mathrm{~J} 2=355^{\circ} .80+1191^{\circ} .3 \mathrm{~T}, \mathrm{~J} 3=119^{\circ} .90+262^{\circ} .1 \mathrm{~T}$, $\mathrm{J} 4=229.80+64.3 \mathrm{~T}, \mathrm{~J} 5=352.25+2382.6 \mathrm{~T}, \mathrm{~J} 6=113.35+6070.0 \mathrm{~T}$

$$
\begin{aligned}
\text { Saturn: Mimas } & =40.66-0.036 \mathrm{~T}+13.56 \sin \mathrm{S} 1 \\
& =83.52-0.004 \mathrm{~T}-1.53 \cos \mathrm{S} 1 \\
& =340.81+381.9945550 \mathrm{~d}-13.48 \sin \mathrm{S} 1-44.85 \sin \mathrm{S} 6 \\
& \\
\text { Enceladus } & 40.66-0.036 \mathrm{~T} \\
& 83.52-1.18+262.7318996 \mathrm{~d} \\
\text { Tethys } & 40.66-0.036 \mathrm{~T}+9.66 \sin \mathrm{S} 2 \\
& =83.52-0.004 \mathrm{~T}-1.09 \mathrm{cos} \mathrm{S} 2 \\
& =10.77+190.6979085 \mathrm{~d}-9.60 \sin \mathrm{S} 2+2.23 \sin \mathrm{S} 6 \\
& =40.66-0.036 \mathrm{~T} \\
\text { Dione } & 83.52-0.004 \mathrm{~T} \\
& =356.68+131.5349316 \mathrm{~d}
\end{aligned}
$$




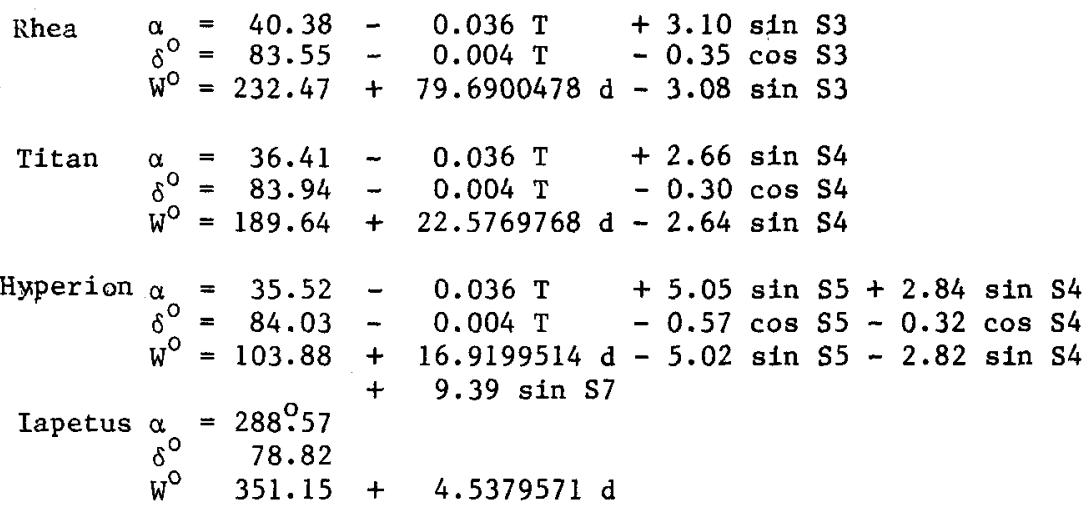

where $\mathrm{S} 1=177^{\circ} .40-3650^{\circ} .5 \mathrm{~T}, \mathrm{~S} 2=300^{\circ} .00-7225^{\circ} \cdot 9 \mathrm{~T}, \mathrm{~S} 3=345^{\circ} .20-1016^{\circ} .3 \mathrm{~T}$, $\mathrm{S} 4=29.80-52.1 \mathrm{~T}, \mathrm{~S} 5=261.45-239.2 \mathrm{~T}, \mathrm{~S} 6=316.45+506.2 \mathrm{~T}$, $\mathrm{S} 7=100.10+20528.5 \mathrm{~T}$

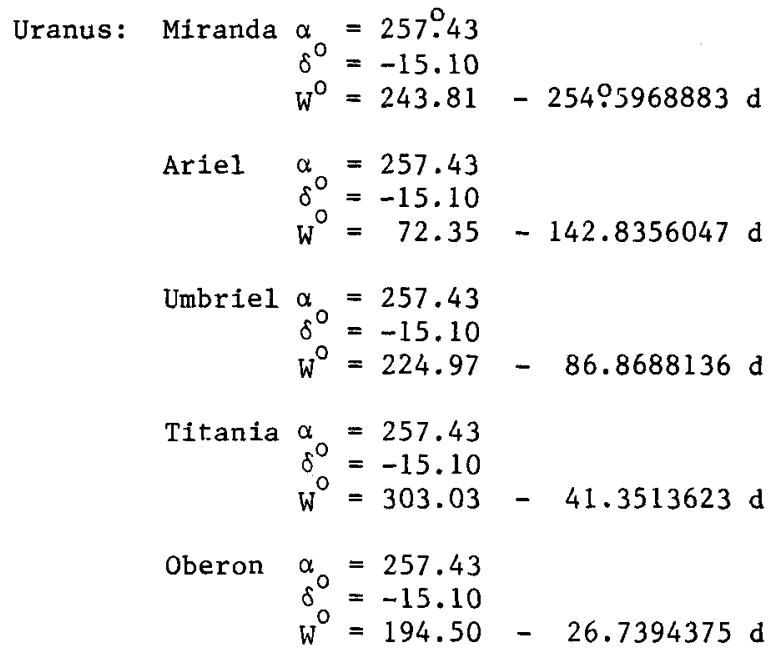

Neptune: Triton $\alpha=295.34-20.12 \sin \mathrm{N}$$$
\delta^{0}=37.05+15.26 \cos \mathrm{N}
$$$$
W^{0}=297.25-61.2575147 \mathrm{~d}+10.56 \sin \mathrm{N}
$$

where $\mathrm{N}=189^{\circ} .66+62^{\circ} .0 \mathrm{~T}$

Pluto: Charon $\alpha_{0}=311.63$

$\delta^{\circ}=4.18$

$W^{\circ}=25.66-56.3640000 d$

Note (a) The $182^{\circ}$ meridian is defined by the crater Cilix.

(b) The $128^{\circ}$ meridian is defined by the crater Anat.

(c) The $326^{\circ}$ meridian is defined by the crater Saga.

(d) The $162^{\circ}$ meridian is defined by the crater Palomides.

(e) The $5^{\circ}$ meridian is defined by the crater Salih.

(f) The $299^{\circ}$ meridian is defined by the crater Arete.

(g) The $63^{\circ}$ meridian is defined by the crater Palinurus.

(h) The $340^{\circ}$ meridian is defined by the crater Tore.

(i) The $196^{\circ}$ meridian is defined by the crater Bahloo 
(j) The $276^{\circ}$ meridian is defined by the crater Almeric.

Table 5. Recommended Reference Spheroids for Mapping the Planets and Major Satellites (1982).

\begin{tabular}{|c|c|c|c|}
\hline \multirow[b]{2}{*}{ Planet } & \multirow[b]{2}{*}{ Satellite } & \multicolumn{2}{|l|}{ Equatorial } \\
\hline & & Radius $(\mathrm{km})$ & Flattening \\
\hline \multirow{4}{*}{$\begin{array}{l}\text { Mercury } \\
\text { Venus } \\
\text { Earth }\end{array}$} & & 2439 & 0 \\
\hline & & 6051 & 0 \\
\hline & & 6378.140 & 0.00335281 \\
\hline & Moon & 1738 & 0 \\
\hline \multirow{6}{*}{$\begin{array}{l}\text { Mars } \\
\text { Jupiter }\end{array}$} & & 3393.4 & 0.0051865 \\
\hline & & 71398 & 0.0648088 \\
\hline & Io & 1815 & 0 \\
\hline & Europa & 1569 & 0 \\
\hline & Ganymede & 2631 & 0 \\
\hline & Callisto & 2400 & 0 \\
\hline \multirow[t]{9}{*}{ Saturn } & & 60000 & 0.1076209 \\
\hline & Mimas & 198 & 0.019 \\
\hline & Enceladus & 253 & 0.020 \\
\hline & Tethys & 525 & 0 \\
\hline & Dione & 560 & 0 \\
\hline & Rhea & 765 & 0 \\
\hline & Titan & 2575 & 0 \\
\hline & Iapetus & 725 & 0 \\
\hline & Phoebe & 110 & 0 \\
\hline \multirow{6}{*}{ Uranus } & & 25400 & 0.030 \\
\hline & Ariel & 400 & 0 \\
\hline & Umbriel & 275 & 0 \\
\hline & Titania & 500 & 0 \\
\hline & Oberon & 450 & 0 \\
\hline & Miranda & 150 & 0 \\
\hline \multirow[t]{2}{*}{ Neptune } & & 24300 & 0.0259 \\
\hline & Triton & 1600 & 0 \\
\hline \multirow[t]{2}{*}{ Pluto } & & 1500 & 0 \\
\hline & Charon & 600 & 0 \\
\hline
\end{tabular}

Note: The equatorial radil for Mercury, Venus, Moor, Mars, Io, Europa, Ganymede, Callisto, Mimas, Enceladus, Tethys, Dione, Rhea, and lapetus are used in current mapping programs, and those for Jupiter and Saturn are used in sequencing and analyzing data from current flight missions. The values for Mars and Pluto differ from those recommended by the IAU in 1976 (Trons. IAU, 16B, p. 60). The reference spheroid for Mars ( $3393.4 \mathrm{~km}$ radius) has been used in all mapping programs since 1973, although the IAU 1976 radius $(3397.2 \mathrm{~km}$ ) is probab1y a better value. In 1976 Pluto's satellite, Charon, had not been discovered.

Table 6. Recommended Reference Shapes for Mapping Irregular Satellites (1982)

\begin{tabular}{|c|c|c|c|c|}
\hline & & Equatorial & Equatorial & Polar \\
\hline Planet & Satellite & Radius, $A(\mathrm{~km})$ & Radius, $B(\mathrm{~km})$ & Radius, C (km) \\
\hline Mars & $\begin{array}{l}\text { Phobos } \\
\text { Delmos }\end{array}$ & $\begin{array}{r}13.5 \\
7.5\end{array}$ & $\begin{array}{r}10.7 \\
6.0\end{array}$ & $\begin{array}{l}9.6 \\
5.5\end{array}$ \\
\hline
\end{tabular}


COMMISSION 16

$\begin{array}{llrrr}\text { Jupiter } & \text { Amalthea } & 140 & 105 & 80 \\ \text { Saturn } & \text { Hyperion } & 200 & 125 & 110 \\ & \text { Janus } & 110 & 95 & 80 \\ & \text { Epimetheus } & 70 & 57 & 50\end{array}$


COMMISSION 19: ROTATION OF THE EARTH (ROTATION DE LA TERRE)

Report of Meetings 18, 19,23 and 24 August 1982

President: P. Paquet Secretary: W.J. K1epczynski

18 August 1982 (Morning)

\begin{abstract}
After the opening of the meeting, the president reported on some of the highlights of the past three years. These included: the availability of the observations made within the People's Republic of China (PRC); the successful completion of the MERIT Short Campaign; and, the establishment of the IAU/IAG Joint Working Group on the establishment and maintenance of a Conventional Terrestrial Reference System (COTES). He emphasized the need to still continue observations with the classical techniques, and noted the improvements which were being incorporated into the classical instrumentation. He also looks forward to the completion of the Hipparcos catalogue and the consequent improvement that should result in some optical determinations of Earth Rotation Parameters (ERP).
\end{abstract}

B. Guinot summarized the parts of the Annual Report of the Bureau International de 1 'Heure which pertained to Commission 19. He stressed the involvement of the BIH in the Merit short Campaign. The BIH acted as the coordinator of the campaign and furnished weekly summaries of ERP's derived through analyses of observations contributed by the new techniques. He also noted the steady improvement in the ERP's published by the BIH over the years. In Polar Motion (PM), the pair variance improved from 0:.015 in 1967 to $0: 006$ in 1982. The most recent values reflect the inclusion of improved Doppler data, observations from the PRC, and Connected Element Radio Interferometry. In Universal Time (UT), the formal standard deviation (not pair variance) has improved from $1.3 \mathrm{~ms}$ in 1967 to about $0.6 \mathrm{~ms}$ in 1982. He pointed out that a weaker smoothing of UT1 was introduced in circular D on 1 January 1982. The new smoothing interval is 30 days as opposed to 90 days. The weaker smoothing is expected to improve the comparison of observations with BIH data by not artificially masking the short period effects. He also stressed the importance of the role that the G.E. Mark III Information system played during the MERIT Short Campaign in allowing fast, efficient international exchange of data.

K. Yokoyama, the new Director of the IPMS, reported on its activity during the last three years. He noted the dissolution of the ILS chain in June 1982 with the termination of observations at Gaithersburg and Ukiah by the National Geodetic Survey. During the MERIT Short Campaign, the IPMS acted as an analysis center for the classical techniques. He reported that the IPMS analysis noted the same short period variations in UT as other new techniques when compared with the strongly smoothed BIH data. He also noted the improvement in precision and accuracy of ERP's derived from optical astrometry due to the inclusion of data from the PRC. The completion of the re-reduction of past ILS observations from 1899 to 1978 was mentioned. 
Ye Shu-hua reported on activities within the PRC. There are now six observatories contributing to Earth rotation studies and observations: Shanghai Observatory (responsible for the issuing of UT1); Purple Mountain Observatory (in Nanjing); Beijing observatory and its Tienjin Latitude station (responsible for Polar Motion); Wuchan Time observatory (affiliated with the Institute of Geodesy and Geophysics); Shaanxi Observatory (responsible for broadcasting $H F$ and LF time signals); and, Yunan observatory (in Kunming). Presently, the internal consistency for UT, determined within the PRC, is about $1 \mathrm{~ms}$ and for PM about 0:01. While three months predictions for UT1 and PM are good to $+5 \mathrm{~ms}$ and +0.01, respectively. Local atomic time scales are maintained at Shanghai, Shaanxi and Beijing observatories. Photoelectric astrolabes are now in operation at Beijing and Shanghai observatories.

S. Debarbat reported on her comparison of Paris observatory Astrolable observations with those of the Green Bank Connected Element Radio interferometer. She found that over the two and a half years of comparison, the external precision of the two techniques remained constant regardless of whether one uses a short or long span of dates. The one day averaged values for the Paris astrolabe have an external precision of $4.5 \mathrm{~ms}$ in UT and 0.059 in PM, while the three day averaged values of the Green Bank Interferometer have an external precision of $2.0 \mathrm{~ms}$ in UT and 0.034 in PM.

R.0. Vincente noted the success of the MERIT Short Campaign and its effective communications. He cautioned that extreme care must be exercised in analyses based on short intervals of data such as the three months of the short Campaign. Furthermore, he recommended that investigators use continuous data points instead of averaged or smoothed values. He showed by several examples that significantly different values of the chandler frequency could be obtained as a function of the time span of the data set used in the analysis.

C.0esterwinter, reporting for R.J. Anderle and C.A. MalyevaC, noted that PM, as determined from the "OCSAR" type Navy Navigation Satellites (TRANSIT) had deteriorated in the 1 ast two years due to increased drag effects arising from the high levels of solar activity. The use of rubidum frequency standards in portable tracking equipment should improve the overall performance of the observing network. A new procedure was introduced to derive UT based on discrepancies of the predicted Right Ascension of the NAVSAT satellites rather than on the basis of the prediction errors in the longitude of the node. While the revised procedure produces useful results, they are still not conclusive.

V. Dehant presented the results of a study, co-authored by P. Paquet, which analyzed the variations in the height of the Brussels station as deduced from Doppler observations during the period 1972-1980. The variations, which are related to unmodelled perturbations of the troposphere and ionosphere, can be interpreted as an estimation of the stability of the Doppler system. The main variations are of medium and long period: an annual component, one of about the 11.75 year period of the solar activity and one of four months (121-128 days). 
J. Fanselow reported on JPL's effort to develop a near real time UT and PM service in support of the Deep Space Network. The nominal operational capability is for weekly measurements of ERP's available within one week after observations with an accuracy of $10-20 \mathrm{cms}$.

B.E. Schutz reported on the work that he and his colleagues, B.D. Tapley and R.J. Eanes, have done at the University of Texas at Austin on deriving ERP's from satellite laser ranging. Presently, the satellite force model LPM81.12 (page D-13, BIH Annual Report 1981) is being improved and a new model is expected to go into use after January 1983. Because UT1 is correlated with the satellite's longitude of the ascending node, UTl changes can only be identified if an adequate model for the nodes exists. By basing a solution for the node on one year of data, one can convert node residuals into UT1 changes. Comparison of these UT1 residuals with weakly smoothed UT1 of BIH show good correlation.

Ye Shu-hua reported on the work of Gu Zhen-nian, Wang Shu-he and Zjeng Da-wei from Shanghai observatory on analyzing the differences in PM deduced from several series of classical optical observations and that deduced from Doppler (DMA) and Satellite laser ranging (IASOM).

\section{August 1982 (Afternoon)}

W. Markowitz noted that it is almost impossible to determine exactiy the periods of the annual and chandler terms of PM. He bases this conclusion on the assumption that the annual and Chandler terms have random variations which preclude their separation and the fact that there are random observational errors further complicates the problem. By using short spans of data, which cover the beat period between the two periods (6-7 years) one can get reasonably good resolution of the two terms.

L.V. Morrison and F.R. Stephenson reported on their computation of the secular and decade fluctuations in the Earth's rotation between 700 BC and $A D 1978$ from timings of lunar eclipses and occultations.

T. Van Flandern reported on the work which he and his colleague M.R. Lukac, did in computing new values of $\Delta T$ back to 1820 utilizing observations of 150,000 lunar occultations. Their analysis indicates that the Earth's rate of rotation changed by three parts in $10^{10}(1 \mathrm{~s} / \mathrm{cy})$ during an interval of less than a year in early 1896 .

M. Feissel showed that a very strong correlation exists between variations in the length of the day and in the angular momentum of the atmosphere. The variations seem to have a period between 50 and 55 days. 
B. Guinot discussed the chandlerian nutation from 1900 to 1980 following the lines of a study published in 1972. Based on the assumption that the annual term of the annual variation remains stable, he showed that a change in the amplitude of the Chandlerian motion occurred in 1980 .

C. Kakuta conjectured that thermal energy dissipation under the crust, caused by earthquakes, might excite variations in the Earth's rotational motion.

S.K. Runcorn cited the importance of astronomical data on fluctuations in the rotation rate of the Earth and on the Chandler wobble because only the astronomical data, along with geomagnetic secular changes, gives evidence of the dynamical processes in the Earth's interior. The westward drift of the magnetic pole reflects the westward drift of the inner core. Furthermore, no quantitively satisfactory explanation has been offered for the irregular changes in the length of the day other than that they represent transfer of angular momemtum between the core and the mantle. It seems that only the outer part of the core interchanges its angular momentum with the mantle over periods of 10-100 years. In addition, the time scale of the irregular changes in the length of day (as presented by Van flandern) is of importance to the geomagnetician. For this is related to the time taken for a magnetohydrodynamic disturbance to travel over a distance in the core comparable to the length of the non-dipole field. It is also related to the free decay time-constant of the semi-conducting mant1e.

C.F. Yoder discussed the free rotational librations of the Earth's inner core. There are six possible free motions of the Earth's inner core. Three involve displacements of the inner core center of mass with respect to the mantle. The remaining three free modes are rotations of the inner core figure with respect to the mantle figure: a chandler-like wobble, a free nutation of the inner core spin axis and a free libration in longitude. The free wobble exists even if no external torques act on the Earth's inner core while the remaining free motions require an external restoring torque. M. Slade and $C$. Yoder found that the gravitational interaction between the figures of the core and the mantle will drive the free nutation and longitude librations and reduce the period of the inner core wobble to about eight years for a density jump of about $0.6 \mathrm{gm} / \mathrm{cm}^{3}$ at the inner core-fluid core boundary. The equivalent free nutation period of the inner core spin axis is about 12 years.

19 August 1982
Joint Meeting with Commission 31 - Project MERIT
(Session I-Chairman, P. Paquet)

Progress reports by the Working Groups on the rotation of the Earth (MERIT) and on the new Conventional TErrestrial reference system (COTES) were presented. 
The first session consisted of three introductory reviews as follows:

G.A. Wilkins:
I.I. Mueller:

M. Feissel:
The objectives and organization of Project MERIT

The objectives and proposals of the Working Group on the conventional terrestrial reference system (COTES)

A review of the MERIT Short Campaign and of the plans for the MERIT Main Campaign $(1983 / 1984)$.

The second session consisted of six reports on the activities and plans for each of the observing techniques and then a consideration of a resolution for submission to the General Assembly. The reports were as follows:

K. Yokoyama:

C. Oesterwinter:

R. Schultz:

0 . Calame:

W.J. Klepczynski:

D.S. Robertson: optical astrometry

Doppler tracking of satellites

Satellite 1 aser ranging

Lunar laser ranging

Connected element radio interferometry

Very long baseline radio interferometry.

The draft resolution was amended in minor respects and then adopted without objection. It was subsequentiy endorsed by the General Assembly.

G. Wilkins, the chairman of the IAU/IUGG Joint Working Group on the Rotation of the Earth explained that Project MERIT is a programme of international collaboration to Monitor Earth Rotation and to Intercompare the Techniques of observation and analysis. A Short Campaign was held in August - October 1980 and the results have been reported in detail. The Main Campaign will take place from 1 September 1983 to 31 0ctober 1984. Current information about the project is distributed in the MERIT Newsletter which is available from the Royal Greenwich Observatory.

I.I. Muelier drew attention to the resolution of IAU Colloquium Number 56 that led to the setting up of the Joint Working Group of which he is chairman; the group is to prepare a proposal for the estabiishment and maintenance of a conventional TErrestrial reference system. For the new system it wiाl be necessary to adopt precise coordinates for a set of well-distributed observatories and to relate the new system to the celestial and inertial systems that are implicit in the various techniques for precise positioning. In particular it will be necessary to determine earth-rotation parameters (ERPs) to a precision of, say, $5 \mathrm{~cm}(0 . .002)$ with a time resolution of 12 hours ( 6 hours if possible). Extra observations will be made during the MERIT Main Campaign to look for shortperiod variations and to obtain data at selected sites by at least two techniques. Several mobile SLR and VLBI systems will be used. The proposals were endorsed by the International Association of Geodesy at its General Meeting in Tokyo in May.

M. Feissel reviewed the MERIT Short Campaign and identified areas which should be improved for the Main Campaign. The purpose of the short Campaign was to test the different observing 
techniques and establish a reliable, efficient communication system. Quick-Look estimates for ERP's were usually available within one week; most results were available within two weeks. Communication of data was done either through the G.E. Mark III Information system, a worldwide computing system, or through TELEX messages. It seemed that the general organization of the short Campaign was satisfactory. In regards to improvements, it is recommended that there be fewer operational Centers and that the coordinators establish better links with the Operational Centers of their respective techniques. Analysis of the data showed that the period covered by the Short Campaign included a reasonably significant perturbation in the path of the pole. Some analyses showed the existence of systematic differences between techniques and also pointed out that different Analysis centers, using the same data, showed systematic differences in ERP's amounting to about $2 \mathrm{~ms}$. This pointed out the need to improve homogeneity in the reduction procedures and the importance of the work of the Standards Committee.

In closing the first session the President of Commission 19 congratulated the Working Groups on the progress that has been made since the last General Assembly.

Please refer to Report of Commission 31 for minutes of Session II.

\section{August 1982}

This session was primarily concerned with new instrumentation. The first three reports dealt with recent improvements to the astrolabe. Two reports on the forthcoming Hipparcos satellite were presented. The session also included reports on Connected Element Radio Interferometry and the Canadian Polar Motion stations.

G. Billaud reported on the photoelectric astrolabe designed and built at CERGA. The design goal was to overcome the three major imperfections of the classical Danjon astrolabe: 1) the temperature induced variation of the angles of the glass prism; 2) the chromatism and astigmatism of the walloston prism images; and, 3) the personal equation of the observer. The instrument has a photomultiplier to replace the observer, a Maksutov-Cassegrain reflector instead of a refractor, and a modified Hog's slit micrometer. Preliminary results obtained from observations made from 9 February - 19 March 1982 indicate an accuracy of $3.8 \mathrm{~ms}$ in UTO-UTC, i.e., standard deviation of a comparison with the BIH.

Z.-y Li of Shanghai Observatory discussed the development of the photoelectric astrolabe within the PRC. A prototype Type I Photoelectric Astrolabe was constructed in 1972 by the Nanjing Astronomical Instrument Factory. It was installed at Shaani observatory. Three Type II instruments were subsequently developed in 1974 and installed at Beijing, Shanghai and Yunan. The Type I: instrument is divided into two parts. One is in the dome and consists of the photomultiplier box and telescope; the other part is located in a room under the dome and consists of the telescope 
controls, amplifiers and power supplies. The main telescope is sealed and exhausted to a low vacuum to eliminate refraction inside the tube and contamination of the mercury. In regard to accuracy, the rms deviation for time is $2.5 \mathrm{~ms}$ when compared to the smoothed Chinese UT1 system and in latitude it is 0.02 when compared to a Vondrak smoothed curve, fit through the observations.

C. Kakuta described the photoelectric astrolabe of Mizusawa. It is a new Tsubokawa automatic astrolabe of a pendulum type with an electronic transit detector. The instrument is based on the same principle as the original one at Kanozan Geodetic observatory. Several improvements have been made and a real time digital filter is designed to eliminate motions of the stars. Presently, the instrument is being evaluated and the observational results will be compared with those derived from the Danjon astrolabe.

J. Popelar reported on three years (1979-1981) of PZT and Tranet satellite Doppler observations at two collocated stations near ottawa and Calgary. A comparison of the variation in station coordinates and relative precision of the two techniques was made. In regard to station longitude, the Calgary PZT shows long term variations in the 3-4 m range while for the ottawa PZT they are below the $2 \mathrm{~m}$ level. The two Tranet stations show long term variations in the horizontal station coordinates at the $2 \mathrm{~m}$ level with no apparent correlation between the stations. However, the height variations exceed $2 \mathrm{~m}$ and are clearly correlated. Residual variations of the 5 day averages from smoothed long term curves yield standard deviations around the $1 \mathrm{~m}$ level for both techniques. Popelar pointed out that careful hardware calibrations and careful modelling are necessary for best Tranet results.

W.J. Klepczynski described improvements to the Green Bank $35 \mathrm{~km}$ interferometer system. A new element, located orthogonal to and $32 \mathrm{~km}$ distant from the present baseline, will be in operation in the fall of 1983. This will allow the interferometer to resolve al1 three components of the Earth's rotation. A new source position catalogue was recently introduced. Using this new catalogue to re-reduce past data obtained during the best observing weather, the internal mean errors for UTO-UTC improved to $0.4 \mathrm{~ms}$. In the near future, a new atmospheric model will be used in the reduction of observations. This should help in reducing the annual term exhibited in comparisons with the BIH. Water vapor radiometers, which will measure the differential water vapor seen along the line-of-sight between two antennas are being constructed and should be in operation within 4-6 months. They will be useful in reducing the day-to-day scatter in UT and PM as determined by the interferometer.

J. Kovalevsky reported on the status of the Hipparcos sateliite. Launch is scheduled for 1986. It is hoped to have the first catalogue, based on Hipparcos observations, completed by 1991. A preliminary source list is scheduled to be completed next year, with final source catalogue to be completed by 1985 . There stili are a few small problem areas. One is the existence of possible systematic errors due to chromatic effects caused by nonalignment of optical parts; the other is the introduction of spurious signals in the detector (glare) caused by the presence of 
a stray bright star in the field of view. By choosing an attitude control system controlled by jets of gas, a possible source of error, called jitter, has been eliminated.

M. Feissel discussed the list of stars selected for the Hipparcos program from those supplied by members of Commission 19. Initially, 10,000 stars were proposed, but the list was cut to 5,508 stars. The majority of the stars are between $10^{\circ} \leqslant \delta \leqslant 60^{\circ}$ and 6-7 magnitude. The stars in this program are to be used primarily for geodetic purposes.

\section{August 1982 (Scientific)}

P. Hill discussed the plans of the Groupe de Rechereche de Geodesie Spatiale (GRGS) for VLBI. Primary aim of the project is for global geodynamics and astrometry. Initial plans call for participation in the ERIC (EISCAT Radio Interferometric Campaign) Project and include the acquisition of two Mark II terminals and a compact French hydrogen maser. Future plans call for the development of a large fixed antenna and a $15 \mathrm{~m}$ transportable antenna with Mark III terminals.

H. Enslin reported on the work of $C$. de Vegt to construct a photographic PZT reference star catalogue which will connect the PZT star catalogues of Calgary, Herstmonceux, Hamburg, Ondrejor and Potsdam. Observations with a $23 \mathrm{~cm}$ astrograph, along a $6^{0}$ belt centered at $52.5 \mathrm{~N}$, have begun. It is planned to adjust the declination system to an extragalactic reference frame through the optical counterparts of 45 compact extragalactic radio sources.

B. Kolaczek reviewed the secular and irregular variations of the mean latitudes of IPMS/BIH stations. She found that the variations of mean latitudes showed some regional similarities. She also computed the local annual term of latitude variations for the period 1962-1980 for the variations in latitude and found that they are very changeable for some stations and more stable for about 25 stations.

Ya. Yatskiv reported on the work of L.D. Kostina and V.I. Sakharov, on the long period variations in the chandler wobble. For the period 1900-1975, they have fit the Chandier wobble by 2 two parameters $(\alpha, \beta)$ exponential functions. For the two exponential functions, they found that the growth coefficientc $\left(\alpha^{\prime} s\right)$ were different, but that the damping coefficients ( $\beta^{\prime} s$ ) were similar. This leads them to conclude that al though there may be different excitation mechanisms, the Earth's damping mechanism has remained stable.

L. Randic has been comparing the latitude differences between pairs of stations in the northern and southern hemispheres. For the pairs, Mizusawa - Mt. Stromlo and Washington - Santiago, he finds that the latitude differences are not constant and appear to exhibit an annual term. He discussed some possible causes for this, among them are meterological effects and geophysical processes. 
W. Markowitz noted the differences between the astronomical and geodetic systems of reckoning PM. In the astronomical system, the $y$ axis is located at $90^{\circ} \mathrm{W}$ longitude with longitudes increasing to the West. In the geodetic system, the $y$ axis is $10 c$ ated at $90^{\circ} \mathrm{E}$ with 1 ongitudes increasing to the East. He noted that it seems harmful to maintain both systems and recommended that astronomers adopt the geodetic system for PM.

P. Paquet summarized a study prepared by C. Dramba which attempts to develop an analytical representation for PM which includes the effects of an elastic Earth.

\section{August 1982 (Business)}

The President, P. Paquet, reported on the election of officers. Ya. Yatskiv was elected President and W.J. Klepczynski, Vice-President. In addition to Yatskiv and Klepczynski, the new organizing committee will be composed of F. Barlier (82), H. Enslin $(80)$, C. Kakuta (80), B. Kolaczek (80), I. Mueller (82), V. Naumov $(80)$, P. Paquet (past President), E. Proverbio (80), M. Rochester (82), Shu-hua Ye (82), and K. Yokoyama (IPMS).

Twenty-three new members were approved into the commission. Two members resigned during the last triennum, J.L. Locke and J.D. Mulholland and two members died, E. Buchar and J.0. Fleckenstein. Three new consultants were added to the commission: A. Stolz (Australia), N. Sidorenkov (USSR), Sh-y. Zhu (PRC). B. Kolaczek was appointed as Commission 19's representative to the Directing Board of the IPMS and H. Ensiin's appointment as Commission $19^{\prime} \mathrm{s}$ representative to FAGS was continued.

Resolution Adopted at the Joint Session of Commissions 19 and 31 on Project MERIT 18 August 1982

For details, refer to report of Commission 31 .

Resolution Adopted at the Joint Session of Commission 19 and 31 on Regularized UT1, 19 August 1982

For details, refer to the report of Commission 31 .

Resolution Adopted at the Joint Session of Commissions 4, 19 and 31 on Nutation and the Definition of GMST, 20 August 1982

For details, refer to the report of Commission 4 .

Resolution Adopted at the Joint Session of Commissions 4,19 and 31 on the System of Astronomical Constants and Ephemerides, 20 August 1982

For details, refer to the report of Commission 4 . 
REPORT OF JOINT MEETING OF COMMISSIONS 19 (ROTATION OF THE EARTH) and 31 (TIME) HELD ON 1982 AUGUST 19.

CHAIRMAN : 1st session (09.30-11.00) P. Pâquet 2nd session (11.30-13.15) S. Iijima

Progress reports by the Working Groups on the rotation of the Earth (MERIT) and on the new conventional terrestrial reference system (COTES).

The first session consisted of three introductory reviews as follows :

G.A. Wilkins : the objectives and organisation of Project MERIT

I.I. Mueller : the objectives and proposals of the Working Group on the conventional terrestrial reference system (COTES)

M. Feissel : a review of the MERIT Short Campaign and of the plans for the MERIT Main Campaign (1983/4).

The second session consisted of six reports on the activities and plans for each of the observing techniques and then a consideration of a resolution for submission to the General Assembly. The reports were as follows :
K. Yokoyama : optical astrometry
C. Oesterwinter : Doppler tracking of satellites
R. Schutz : satelitite laser ranging
O. Calame : lunar laser ranging
W.J. Klepczynski: corrected-elements radio interferometry
D.S. Robertson : very-long baseline radio interferometry.

The draft resolution was amended in minor respects and then adopted without objection. It was subsequently endorsed by the General Assembly and is given as resolution No. $\mathrm{R} 5$ on page 39.

Wilkins, the chairman of the IAU/IUGG Joint Working Group on the Rotation of the Earth explained that Project MERIT is a programme of international collaboration to monitor earth rotation and to intercompare the techniques of observation and analysis. A Short Campaign was held $\overline{i n} 1980$ August to October and the results have been reported in detail (Wilkins and Feissel, 1982). The Main Campaign will take place from 1983 September 1 to 1984 october 30. Current information about the project is distributed in the MERIT Newsletter which is available from the Royal Greenwich Observatory.

Mueller drew attention to the resolution of IAU Colloquium No. 56 (Gaposchkin and Kolaczek, 1981) that led to the setting up of the Joint Working Group of which he is chairman; the group is to prepare a proposal for the establishment and maintenance of a conventional terrestrial reference system. For the new system it will be necessary to adopt precise coordinates for a set of well-distributed observatories and to relate the new system to the celestial and inertial systems which are implicit in the various techniques for precise positioning. In particular it will be necessary to determine earth-rotation parameters (ERPs) to a precision of, say, 5cm (0".002) with a time resolution of 12 hours ( 6 hours if possible). Extra observations will be made during the MERIT Main Campaign to look for short-period variations and to obtain data at selected sites by at least two techniques. Several mobile SLR and VLBI systems will be used. The proposals (COTES, 1982) were endorsed by the International Association of Geodesy at its General Meeting in Tokyo in May. 
Feissel summarised the principal conclusions that had been drawn from the MERIT Short Campaign, which had stimulated faster development of the new techniques and had shown where improvements are required in the operational procedures. In order to bring the reference systems into better agreement it will be necessary to adopt a standard set of constants and models and to go on looking for sources of systematic errors in the techniques. Special efforts are also needed to eliminate the "weekend effect", to improve the links between the operational and coordinating centres, and to disseminate the information more widely, as well as to look for short-period variations and to make colocated observations.

In closing the first session the President of Commission 19 congratulated the Working Groups on the progress that has been made since the last General Assembly.

Yokoyama pointed out the Short Campaign had led to an improvement in the results from optical astrometry and that a further improvement could be expected during the Main Campaign as a result of the use of more accurate star catalogues for the astrolabes and PZTs and better corrections for nutation and tidal effects. He hopes to determine short-period variations from the observations at the better stations.

Oesterwinter reported that data from the Doppler tracking of satellites had been analysed in an attempt to detect plate motions; the values found for the Australian and Pacific plates appeared to be significant and in general agreement with geological studies. The lack of proper information about local changes in the positions of the antennae had hindered the analyses. In reply to a question, Guinot stated that a new MEDOC II system is being developed; it will include four new French stations and should come into operation during the Main Campaign.

Schutz reviewed the current status of the Satellite laser ranging stations that supplied data to the analysis centres at Greenbelt and Austin in the USA. For many stations the precision is about $10 \mathrm{~cm}$ and for one or two it is $2-3 \mathrm{~cm}$. There is a very noticeable weekend effect in the frequency of observations; a better balance between day and night observations will also be required if reliable determinations of short-period variations are to be made. More effort must be made to reduce both systematic and random errors. Guinot commented that the monthly reports from the University of Texas are very useful.

Calame said that the lunar laser ranging contribution to MERIT was carried out in the framework of the EROLD campaign, but was limited to the determination of UT since data were only available from the McDonald Observatory. She expressed the hope that LLR data would also be available from Grasse, Wettzell and Orroral during the Main Campaign, and she presented solutions for the annual and semi-annual terms in UT from McDonald data for the period 1971-1981.

Klepczynski spoke mainly about the connected-element radio interferometer at Green Bank since this was the only CERI system in regular use for the determination of earth-rotation parameters. Some results had been obtained from Cambridge, England, during the Short Campaign and he hoped that some time would be available on the Very Large Array at Socorro during the Main Campaign. The Green Bank system is being extended to allow the determination of all three parameters, and a water-vapour radiometer is being installed in order to reduce the errors due to the troposphere. In response to a question he said that it might be possible to obtain a solution with a precision of $0.5 \mathrm{~ms}$ at an interval of 8 hours.

Robertson showed results from VLBI and claimed that UT could be obtained with a precision of the order of $0.1 \mathrm{~ms}$ from 24 hours of observations in spite of bad weather and bad ionospheric conditions. The POLARIS network in the USA requires one more element in order to obtain all three parameters; observations will be made every five days. In response to a question he said that this network when used with another element at Wettze11, Germany, should give results with precisions of $0.1 \mathrm{~ms}$ and $5 \mathrm{~cm}$ from 24 hours of observation. 
In the ensuing general discussion Wilkins stated that the third and final draft of the MERIT recommendations on standards should be available during the autumn and that recommendations on the monumentation of sites could be prepared since this will be very important in the establishment of the new conventional terrestrial reference frame.

The meeting then adopted a resolution that noted the success of the MERIT Short Campaign, recognised the potential value of the MERIT Main Campaign, and endorsed the two resolutions concerning the COTES proposals for extra observations that were adopted recently by IAG. (See Resolution No. R5 on page 40.) The President of Commission 31 closed the meeting by thanking all those who had presented reports and contributed to the discussions.

\section{References}

COTES, 1982. Reference Frame Requirements and the MERIT Campaign : proposal for extra observations. Special issue of CSTG Bulletin.

Gaposchkin, E.M. and B. Kolaczek (eds), 1981. Reference Coordinate Systems for Earth Dynamics. D. Reidel Publishing Co., Dordrecht, Holland.

Wilkins, G.A. and M. Feissel (eds), 1982. Project MERIT : Report on the Short Campaign and Grasse Workshop with observations and results on earth-rotation during 1980 August-October. Royal Greenwich Observatory, Herstmonceux, Sussex, UK. 
COMMISSION 20: POSITIONS AND MOTIONS OF MINOR PLANETS, COMETS, AND SATELLITES (POSITIONS ET MOUVEMENTS DES PETITES PLANETES, DES COMETES ET DES SATELLITES)

Report of Meetings, 18, 20, and 25 August 1982

PRESIDENT: E Roemer (Acting) SECRETARY: C J van Houten

\section{August 1982}

\section{ADMINISTRATION I}

The meeting was opened by the Vice President, who noted that because of unusual circumstances, the President of the Commission, G Sitarski, had been unable to participate in the planning for meetings of the Comission at the General Assembly, and was now unable to be present. Those in attendance were invited to join in extending greetings to him by signing a card. C J van Houten was then confirmed as secretary, and it was agreed that no interpreter was needed. All present stood in silent respect as the names of three members or former members of the Commission who had died since the previous General Assembly were read: P Herget, H Hirose, J G Porter.

Proposed new officers were $E$ Roemer as President and $C J$ van Houten as Vice President. Working Group chairmen proposed as continuing for the 1982-85 triennium were L Kresák, comets; Y Kozai, satellites; and G E Taylor, prediction of occultations. Proposed members of the new Organizing Committee were Yu V Batrakov, M P Candy, Y Kozai, L Kresák, L K Kristensen, B G Marsden, H Scholl, G E Taylor, D K Yeomans and $Y Z$ Zhang. The names of a number of individuals who had been nominated as new members or consultants of the Commission were then announced; in some instances, eligibility would depend on confirmation of membership in the IAU. Additional nominations could still be considered, with the list of new members and consultants to be finalized at a later meeting of the Commission. The chairman then announced that a subvention of $10000 \mathrm{SwFr}$ for the Minor Planet Center was included in the proposed IAU budget for the triennium; approval was hoped for.

The chairman then reported on negotiations in progress with the Working Group on Planetary System Nomenclature (WGPSN) with the aim of clarifying the allocation of responsibilities for assignment of designations and names to bodies in the solar system. Responsibility for designations and names of satellites had grown into a matter of considerable concern to some members of Commission 20 . At the request of the chairman, Roemer, Kozai and $\mathrm{K}$ Aksnes were confirmed as representatives of the Commission on a committee that would try to develop a plan responsive to concerns of WGPSN, Commission 20, and Commission 6.

Several recommendations were in process of refinement and would be presented for Commission consideration at a later administrative session. Topics addressed included a proposal to give a limited review to all proposed new minor planet names, recommendations by the ad hoc comittees on Minor Planet Programs and on Magnitude Ephemerides of Minor Planets, and support for further efforts to recover lost numbered minor planets. On this last topic, it was noted that with the reobservation of (1009) Sirene by $J$ B Gibson at Palomar, in close agreement with the prediction by $\mathrm{L} K \mathrm{~K}$ Kistensen, only six numbered planets were still lost.

\section{SCIENTIFIC SESSION: Comets}

L Kresák, chairman of the Working Group on Comets, opened the session with a brief overview of progress since completion of the triennial report of the Commis- 
sion. Among the most noteworthy events were the recoveries of two long-lost comets, $\mathrm{P} / \mathrm{du}$ Toit-Hartley $(1945 \mathrm{II}=198 \mathrm{2b}, 1982 \mathrm{c})$ and $\mathrm{P} /$ Peters-Hartley $(1846 \mathrm{VI}=198 \mathrm{2h})$, both with the UK Schmidt telescope, Siding Spring.

$B$ G Marsden then reported on the paucity of astrometric observations of comets, noting that during the past few years there has been a dramatic reduction in the number of astrometric observations made of comets. The problem applies not just to faint comets but to bright ones as well. It exists in both hemispheres, but, if anything, the Southern Hemisphere seems to be in slightly better shape than the Northern. The reduction in number of observations seems to coincide with the transfer of publication of observations from the IAU Circulars to the MPCs. Early observations of new discoveries and of recoveries are still published on the IAU Circulars, but with the publication of both series of circulars at the same location, it is clearly more efficient to include the bulk of the observations of comets along with those of minor planets. This facilitates the collection of data in machinereadable form. Files of comet observations were, in fact, included in the version of the magnetic tape of minor planet observations issued in March 1982. The MPCs do not enjoy the prominence given to the IAU Circulars, with their appearance on bulletin boards at observatories around the world, but they do have a moderately large circulation, are still published rather rapidly, and--as far as observations are concerned--have been declared as a 'refereed journal'. Nowadays, dozens of astronomers in other disciplines--radio astronomers, those working with the International Ultraviolet Explorer, even those attempting to bounce radar pulses off comets--are plaguing the Minor Planet Center/Central Telegram Bureau for improved orbit/ephemeris data on comets--data that cannot be supplied if astrometric observations are not made and reported quickly.

Marsden next called attention to nomenclature problems that attend the discovery of comets found long after the actual time of observation. The recent announcement of the discovery of two sungrazing comets in the SOLWIND satellite/coronagraph data of 1981 has created a minor nomenclature problem. Since its introduction in 1870 , the system of provisional letter designations has never been applied in retrospect. If orbits can be determined for comets reported belatedly, those comets have been incorporated in the definitive Roman numeral designation system. But the 1981 Roman numerals will not be assigned until 1983. The letter-designation system could obviously be extended, but there is some question as to whether the year of discovery or of the original observation should be used. Further, if designations are to be given in retrospect, it will be difficult to control the system. Provisional designations could clearly be given to the comets recently identified on the Palomar Sky Survey, but what is to be done about the unconfirmed comet reports that occasionally appear in the IAU Circulars and other literature? It was proposed that a special committee of Comission 20 study the matter, prepare and publish a list of provisional designations, and continue to monitor the situation in the future.

$Z$ Sekanina then gave a brief scientific report concerning his recent studies of Comet Howard-Koomen-Michels (1979 XI) and P/du Toit-Hartley. A satisfactory orbital solution was presented for comet $1979 \mathrm{XI}$, showing that it did indeed fall into the sun. The surviving dust tail was studied as a function of the particie production vs. heliocentric distance, the span of accelerations by solar radiation pressure, and particle-sublimation effects. Extremely refractory material was reported to exist in the submicron range of particle sizes. The motions and brightness variations of the two nuclei of $\mathrm{P} / \mathrm{du}$ Toit-Hartley $(1982 \mathrm{~b}, \mathrm{c}$ ) were inyestigated on the basis of Sekanina's model of split comets. The fragments, $1 \times 10^{6} \mathrm{~km}$ apart at the time of discovery, were found to have separated shortly before perihelion passage during the comet's (unobserved) 1976/77 return to the sun, with a relative nongravitational acceleration near $3 \times 10^{-5}$ of the solar attraction. The anomalous brightness behavior of the companion nucleus at the time of discovery may have been a result of the object's highly oblate shape and morphologically heterogeneous surface. 
D $\mathrm{K}$ Yeomans invited the participation of astrometric observers in the astrometry network of the International Halley Watch (IHW). Five spacecraft, none of which has any internal guidance capability, will require information from groundbased astrometric observations for midcourse corrections. Fast astrometry will be required at certain critical times in the missions. Several meetings were being held at the General Assembly to acquaint potential collaborators with the goals of IHW. D W Dunham then called attention to the need for early groundbased astrometry for guidance also of the ISEE 3 spacecraft, which is to be redirected for a rendezvous in September 1985 with $\mathrm{P} /$ Giacobini-Zinner.

\section{SCIENTIFIC SESSION: Satellites}

$Y$ Kozai, chairman of the Working Group on Satellites, opened the session by distributing copies of a progress report, which was an extension of that published in Trans. IAU XVIIIA, material presented to Commission 7 and notes about very recent work having been added. Many faint satellites as well as faint rings for Jupiter and Saturn were discovered through both ground-based and spacecraft observations in the past three years. Most of the newly discovered satellites have very interesting dynamical characteristics: there are two co-orbital pairs, two seem to shepherd the F ring of Saturn, and three are moving near the equilateral triangular points of Dione and Tethys. Many astrometric observations of satellites with respect to the centers of planets or with respect to other satellites were made and published. Also, mutual phenomena which took place in 1979 and 1980 for Jupiter and saturn were observed and analyzed to determine accurate orbital elements. Motions of the Galilean and other satellites still attract the interest of theoreticians, and several new theories which well represent observations were developed and published. A number of papers addressed the question of the dynamical evolution of satellites, particularly how so many commensurable relations of the mean motions might have arisen through secular changes in orbital semimajor axes.

$S$ Ferraz-Mello then reported on the current status of studies of the motion of the Galilean satellites. The minimal standard residuals in the coordinates of the Galilean satellites that have been obtained from photographic observations correspond to a standard error for a single exposure of $3 \mu \mathrm{m}$ on the plates. To obtain the necessary precision ( $100-200 \mathrm{~km}$ ), multiple exposures ( 4 or more) and long-focus telescopes ( $6 \mathrm{~m}$ or more) are needed. Mutual events may also be observed with such a precision, and hope exists for good results in 1985, when many events will be observable. The best current ephemerides are those obtained with the Sampson-Lieske theory and Lieske's elements E-2. Progress is being made toward development of new theories. Substantial improvement, however, remains strongly tied to the existence of good observations and to the continuity of the present observational efforts.

$P K$ Seidelmann, with the collaboration of $R S$ Harrington, assessed the current understanding of the dynamics of the recently discovered small satellites of Jupiter and Saturn. Some of the satellites had been discovered during the Voyager 1 and 2 encounters, while the 1980 ring plane crossing of Saturn provided both additional Earth-based discoveries and clarification of observations made at the ring-plane passage in 1966. Only isolated observations have been made of some satellites, with the consequence that accurate orbits cannot be determined. A number of satellites are in librational motion, sharing orbits with large and small satellites. Additional observations are required for determination of the librational periods. The dynamics of the small satellites and of ring particles present some interesting and challenging problems in celestial mechanics.

It was noted in discussion that several of the satellites discovered during the Voyager encounters have been observed, or should be observable, from the Earth. The Space Telescope Wide-Field/Planetary Camera with a CCD detector has been used successfully by at least two groups for observations of faint satellites, and $\mathrm{Ch}$. Veillet has recorded several of the small Saturnian satellites photographically with the Danish-ESO $1.5-\mathrm{m}$ reflector at La Silla. 
Marsden then called attention to a problem concerning publication of astrometric observations of satellites, in that from time to time the Minor planet center/ Central Telegram Bureau receives long lists of astrometric observations of satellites. Reports of new discoveries of satellites are published rapidly in the IAU Circulars as a matter of course. Some of the other observations have been published there also, but it is clearly inappropriate to continue this practice or to utilize the MPCs. It is proposed that the Working Group on Satellites examine the situation with respect to the publication of observations of satellites, specifically with regard to the possibility of issuing a companion publication to the MPCs. Such a publication could facilitate the collection of observations in machine-readable form. It could also serve as a forum for publishing orbital data on sateliites and could play a useful role in making the decision as to if and when a new discovery should be given a permanent designation.

On behalf of $J \mathrm{D}$ Mulholland, Roemer called attention to problems posed by collections of astrometric plates of satellites for which funds for measurement and reduction were lacking. In principle, unmeasured plates from Mulholland's collection are available for loan to responsible individuals with reasonable ideas for their use. It is anticipated that a 'catalog' will eventually be compiled so that potentially interested individuals might be better aware of what is available.

\section{August 1982}

\section{ADMINISTRATION II}

Roemer presided over a special administrative session convened to consider recommendations concerning nomenclature developed by the special committee composed of representatives of Commissions 6 and 20 and of WGPSN. A scheme had been worked out to clarify the allocation of responsibilities for the several steps in the assignment of temporary and permanent designations, and, eventualiy, of names to newly discovered bodies in the solar system, including satellites, minor planets, comets, and rings. The long-standing responsibilities of Commission 20 with respect to designations and names for minor planets and comets were affirmed in the scheme. In addition, Commission 20 would be responsible for decisions concerning assignment of permanent designations to satellites and for indicating thereby when assignment of names by WGPSN was appropriate. The proposed scheme was approved without dissent.

[Following ratification by members of Commissions 6 and 20 and of WGPSN, the scheme was submitted to and received the approval of the IAU Executive Committee. Details of the scheme are included in IAU Information Bulletin No. 49, January 1983, and in the Astronomers' Handbook, Section 4 of this volume.]

Roemer then reported on recommendations concerning nomes for recently discovered satellites of Jupiter and Saturn that had been developed by WGPSN. Following considerable discussion, the Commission voted unanimously to recommend permanent designations and to endorse proposed names as follows:

$$
\text { J XIV* } 1979 \text { Ja Thebe }
$$

$\begin{array}{lll}\text { S X } & 1980 & \text { S1 } \\ \text { S XI } & 1980 & \text { S3 } \\ \text { S XII } & 1980 & \text { S6 } \\ \text { S XIII } & 1980 & \text { S13 } \\ \text { S XIV } & 1980 & \text { S25 }\end{array}$

Janus

(*Note that this Roman numeral designation differs from that recommended S XIV $\quad 1980 \mathrm{~S} 25$

Epimetheus

("Dione B")

Telesto

Calypso

The Commission also voted, with one dissent, to support the recommendation of those most knowledgeable about the dynamical studies, that assignment of permanent designations and names for the following satellites was not yet timely: $1979 \mathrm{Jl}, 1979$ $\mathrm{J} 3$, and $1980 \mathrm{~S} 28$.

SCIENTIFIC SESSION: Minor Planets

B G Marsden, Director of the Minor Planet Center, opened the session with a 
report on the status of work on minor planets:

(1) Observations. In contrast to the situation with comets, there is currently no shortage of accurate astrometric observations of minor planets. The August 1982 monthly batch of MPCs contains almost 4500 observations, and the accompanying 223 preliminary orbits amply document the value of several important programs. One can, however, question the usefulness of observations of very faint objects when no effort is made to follow objects up a month later; the limiting magnitude of the program at the Crimean Astrophysical Observatory, $B=18.0$, might be taken as the general limiting magnitude for useful observing programs, unless they are conducted on the scale of the Palomar-Leiden survey and some of the recent Palomar-UK Schmidt surveys by $\mathrm{E}$ Helin and $\mathrm{S} J$ Bus.

(2) Identifications. There is also no lack of activity in finding and establishing identifications. These have led to the numbering of no fewer than 300 new minor planets during the past twelve months. L D Schmadel has recently found more than 2000 previously unsuspected identifications with numbered minor planets. Several observatories have extensive files of old plates; measurement of positions of unnumbered minor planets for which only approximate positions have been available would facilitate the recognition of further identifications.

(3) Lost planets. The above-mentioned activity has reduced the number of lost numbered minor planets to six: 473, 719, 724, 878, 1026 and 1179.

(4) Catalogues. The Minor Planet Center has recently published updates for the 1961 catalogue of orbits of unnumbered minor planets and the 1963 edition of the 'Identifiziemungsnachweis'. The new orbit catalogue contains 2471 entries, the listing of discoveries, 29157 provisional designations, of which 8550 are identified with numbered objects.

(5) Palomar-Leiden and McDonald surveys. The second (and final) phase of the P-L survey is now essentially complete. Of the 516 originally unidentified objects in the McDonald survey, $239(46 \%)$ are now numbered or have been identified with numbered objects; of the 27 such objects brighter than $B=15,18$ are numbered.

(6) Verification. H Scholl has recently derived osculating elements for some 2300 minor planets at 200-day intervals from 1882 to 2017 . One of the uses of this calculation is the checking of all the observations. Residuals have been calculated for the 206329 observations of numbered minor planets in the Minor Planet Center's machine-readable file, and cases larger than 100" have been isolated.

(7) Ephemerides. Since many users now calculate their own ephemerides and procedures for setting telescopes directly from osculating elements, some thought might be given to abbreviating, possibly even eliminating, ephemerides, particularly the quadrature-to-quadrature ones given in detail, only three to a page, in the MPCs. Possibly the information on distances and magnitudes could be shortened, so that six ephemerides could appear on one page. It should be noted that ephemerides have two uses: one is to enable observations to be made of a specific object, and the other is to identify in retrospect whatever minor planet trails may appear on an exposure. Because of the second use, it would be rather unsatisfactory to represent ephemerides using Chebychev polynomials; in any case, the calculation of an unperturbed ephemeris from readily available osculating elements nowadays involves scarcely more effort than interpolating in a published ephemeris.

A straw vote showed a nearly even split between those who preferred that publication of ephemerides in the present form be continued in the MPCs and EMP and those who were prepared to compute their own ephemerides from osculating elements. There was little support for the use of Chebychev polynomials. A suggestion that approximate values of the elements $a, e$ and $i$ be included with the ephemerides in 
the MPCs was warmly received.

$J$ Kovalevsky then presented the report of the ad hoc Committee on Program Definition. After some discussion, a study session was scheduled; a refined version of the recommendations of the committee was adopted by the Commission and is included in the report of the final administrative session.

E Bowell reported on the findings of the ad hoc Comittee on Magnitude Ephemerides of Minor Planets. Iively discussion prior to the Patras meeting, together with a work session in Patras, made it clear that it was premature to make a recommendation to the Commission for adoption in 1982. Consequently, the committee requested that a decision be deferred until 1985. There was agreement that the calculation of minor planet magnitudes should be made by means of an easily applied two-parameter formula that includes the opposition effect as well as a non-inear drop-off in brightness at large phase angles, and that applies to both minor planets and satellites. In order to increase the range of expertise within the ad hoc committee, co-option of several additional members was recommended. Pending adoption of a new system, observers were urged to give all results in the current IAU system in addition to whatever alternative system they might choose to use.

$C J$ van Houten reported on the completion of the Palomar-Leiden survey. The original aim of this survey was to provide statistical data on the orbits of minor planets of magnitude between 16 and 20 in opposition; these results have been published. During the first Tucson colloquium on minor planets, it appeared, however, that the interest in the orbits themselves was considerable. It was decided, therefore, to improve these orbits as much as possible, by improving the original positions and searching for additional positions on the PLS plates. In addition, the plates containing the photometric calibration field were also blinked and yielded 169 orbits. The present number of PLS orbits is close to 2400 , 144 new first-class orbits having been added. The program is near completion.

I van Houten-Groeneveld provided information on the progress of the 1977 Trojan campaign. A strip centered on and perpendicular to the ecliptic had been photographed by $T$ Gehrels with the 1.2-m Schmidt telescope of the Palomar Observatory. The center of the field was near the following libration point of the Sun-Jupiter system, at a distance on the sky of $65^{\circ}$ from Jupiter. The plates of the two central epochs have been blinked, yielding discovery of 36 Trojan and Hilda objects. The number of other minor planets found totals about 1800 . The available plates allow an extension of the observed arc to 19 days; orbital elements will be determined.

$\mathrm{H}$ Scholl then described the opportunity for accurate astrometric observations of minor planets offered by the satellite HIPPARCOS. Objects brighter than $B=13$ can be observed close to quadratures during the interval 1987-1990. The accuracy of minor planet positions determined by HIPPARCOS is expected to be in the range 0.01-0".1. The minor planets $1,2,3,4,6,7,8,9,10,11,14,15,16,18,20$ and 29 can be observed at each quadrature. In addition, the minor planets 5, 13, 19, $22,23,28,31,39,40,42,44,51,63,88,89,192,216,324,349,354,471,511$, 532 and 704 can be observed at least at two quadratures. Ephemerides with an accuracy of $l^{\prime \prime}$ will be needed for those minor planets accepted by ESA for observation by HIPPARCOS. Observers are therefore requested to observe the listed minor planets as often as possible. The purposes of the accurate observations of minor planets include (1) improvement of orbits, (2) determination of the equator and equinox in the HIPPARCOS star catalogue, (3) detection of a possible rotation in the HIPPARCOS star system, and (4) determination of the masses of (20) Massalia and (44) Nysa. In addition, there is the possibility of obtaining accurate orbits of interesting minor planets indirectly, in that HIPPARCOS may observe a sufficiently large number of suitable reference stars. Reference stars for the minor planets 348, 486, 526, 1023 and 1825 should be observed by HIPPARCOS. With accurate orbits for these minor planets, masses for the minor planets 1, 4, 65, 52, and 10, respectively, should 
be derivable.

D I Matson reported on the opportunity for observations of minor planets presented by the Infrared Astronomy Satellite (IRAS). The mission of the satellite, scheduled for launch in late 1982 or early 1983, is to map the celestial sphere at wavelengths of $10,20,50$ and $100 \mu \mathrm{m}$. In the course of the survey, IRAS is expected to make 180000 observations of thermal emission from some 30000 minor planets. Positional accuracy, however, will be between $\sim 0.5$ and $\sim 1$ arcminute, depending upon how many of the satellite's sensors detect each asteroid during a given observation. In addition to the many main-belt asteroids expected to be observed, nearEarth, fast-moving objects will also be discovered. It is urgent that ground-based astrometric observers obtain follow-up observations promptly, so that orbits for these objects can be determined.

After some brief remarks about problems that had arisen concerning names proposed for minor planets, Marsden recommended that the Commission adopt a policy calling for review of all names proposed for minor planets, even when names are proposed by discoverers. The review could be done as indicated in the 1979 commission 20 resolution, except that in the case of a name proposed by the discoverer, the waiting period for naming a newly numbered object could be reduced. He also recommended that the length of names be limited to sixteen characters, including spaces and hyphens. To save possible embarrassment, all who propose names for minor planets were urged to be cautious about situations that could lead to premature publicity.

\section{August 1982}

\section{SCIENTIFIC SESSION: Prediction of Occultations}

The chairman of the Working Group on Occultations, G E Taylor, noted in his opening remarks that despite a considerable effort from both professional and amateur observers the occultation campaigns had only limited success during the preceding three years. Although occultations by 13 minor planets had been observed since 1979, in many cases only one chord had been observed, or chords on only one half of the disk of a minor planet. Lack of adequate fast astrometry in the Southern Hemisphere was a factor. Participation of more observers able to provide precise relative positions of minor planet and star as soon as they are in the same astrometric field could increase the success rate, as could the participation of more ouservers with transportable equipment for photoelectric timing. With information about the larger satellites of Jupiter and Seturn available from the Voyager encounters, the value of predictions of future occultations by these bodies seems limited.

R L Millis made a number of suggestions concerning improvement of occultation timings. Since photoelectric observations are much to be preferred over visual ones, and results from permenent observatories do not give enough information, the participation of many properly equipped mobile observers is essential. Inexpensive systems that give timings to 0.01 precision recorded on magnetic tape and usable on Celestron telescopes of 8- to 14 -inch aperture are being developed at the Lowell Observatory. Inquiries concerning these systems are invited. For the foreseeable future, however, visual observers will far outnumber those prepared to make photoelectric observations, and their participation continues to be useful.

L Wasserman considered how the limited manpower available for observation of occultations could best be used. Some selection criteria must be applied to predicted events by the observers, if not by the predictors. Top priority should be given to occultations by minor planets of large linear or angular size and to events chosen on the basis of expected duration, relative brightness of star and minor planet, or small amplitude of the lightcurve as an index of regular shape. Searches should be made for occultations by particularly important minor planets that involve stars fainter than those found in typical astrometric catalogs. And more help is needed in last minute astrometric refinement of predicted occultation ground tracks. 
E Bowell then reported on occultations of stars by comets. Rather than attempt to observe an occultation by a cometary nucleus, for which the chances of success are slight, it should be of great interest to determine the distribution of material in the coma, in a region close to the nucleus, by measuring directly the optical depth in the continum. Some information on the single-particle scattering function might also be inferred from such measurements. Extended, dusty comets are good candidates for such observations. The astrometric accuracy required is not as great as that for occultations of stars by minor planets because most cometary comae are much larger and therefore produce a wider ground track. A plea was made for early recovery of comets so that an ephemeris of $1^{\prime \prime}$ accuracy could be derived and used to make timely predictions of occultations.

L K Kristensen requested as many observations as possible of occultations of stars by (5I) Nemausa. The purpose is to determine the rotation in the proper motion systems of the future fundamental catalogs that will be based on space astrometry. occultations provide precise observations of planet positions for a period of time longer than the mission length of the HIPPARCOS satellite if the occulted stars have been on the observing program of the satellite. Occultations by (51) Nemausa until the year 2017 have been found by $G E$ Taylor. A current campaign of lightcurve observations will, ultimately, give rotation and flattening of (51) Nemausa, which will increase the accuracy of the derived star positions.

\section{ADMINISTRATION III}

Roemer then presided over the final session of the Commission, at which a number of administrative decisions were finalized. Officers and members of the organizing Committee were confirmed in accordance with nominations announced at the first session of the Commission. New members admitted by vote of the Commission include M F A'Hearn, N A Belyaev, D Benest, R Bien, F Börngen, R I Branham, A Camusi, E Helin, P D Hemenway, J Hers, A S Khatsiashvili, C-I Lagerkvist, $N$ L Lomb, A M Nobili, $P R$ Weissman, and I P Williams. ( $J-X$ Zhang had been co-opted to membership during the triennium, upon full adherence of China to the IAU.) Consultants for 1982-85 were confirmed as follows: C M Bardwell, $\mathrm{K}$ I Churyumov, $\mathrm{R} W$ Farquher, $V$ A Izvekov, Z M Pereyra, C F Peters, V Protitch-Benishek and H J Reitsema.

Composition of Working Groups was confirmed as follows:

Orbits and Ephemerides of Comets: J C Bennett, M P Candy, A C Gilmore, E I Kazimirchak-Polonskaja, İ Kresák (chm), B G Mersden, E Roemer, G Sitarski, Ko Tomita, $\mathrm{R}$ M West, $\mathrm{P}$ Wild and $\mathrm{D} K$ Yeomans.

Satellites: K Aksnes, Yu V Batrakov, A Bec-Borsenberger, O Calame, C Cristescu, S Ferraz-Mello, $R$ Greenberg, P A Ianna, Y Kozai (chm), J H Lieske, R L Millis, B Morando, J D Mulholland, D Pascu, M Rapaport, E Roemer, J L Sagnier, P K Seidelmann, $\mathrm{V}$ A Shor, A T Sinclair, D T Vu; J E Arlot (consultant).

Prediction of occultations: M P Candy, D W Dunham, J L Elliot, A C Gilmore, J Hers, A R Klemola, Y Kozai, B Morando, V A Shor, G E Taylor (chm), L H Wasserman, R M West; H J Reitsema (consultant).

The membership of the ad hoc Committee on Magnitude Ephemerides of Minor Planets (and satellites), augmented to include representation from Commissions 15 and 16 , was approved as follows: Yu V Batrakov, E Bowell (chm), C Chapman, $\mathrm{T}$ Gehrels, A Harris, B Hafke, L Kresák, B G Marsden, D Morrison, E Roemer, I D Schmadel and J Veverka. The committee will examine various formulae that meet the several criteria specified in the interim report and recommend a formula for the prediction of magnitudes to be considered for adoption by the IAU in 1985.

Establishment of a Satellite Nomenclature Liaison Committee (SNLC) was then formally approved. This committee will work with WGPSN on matters of satellite nomenclature. The membership is to include the President and vice President of the 
Commission as well as the Chaiman and two other members of the Working Group on Satelites. The members of the committee for $1982-85$ are $E$ Roemer, $C J$ van Houten, $Y$ Kozai, K Aksnes and P K Seidelmann. Aksnes will serve as chairman and occupy the voting seat on WGPSN accorded to a representative of Commission 20; Seidelmann was designated as his alternate. This committee also was asked to consider the style of the permanent designations for satellites, some preference having been expressed in WGPSN for Arabic rather than Roman numerals. Following some discussion of the merits of the alternatives, a straw vote of Commission 20 members showed a strong preference for Roman numerals over Arabic.

Formation of a Comet Nomenclature Committee (CNC) was also opproved, with membership to include the President of the Commission (Roemer), the Chairman of the Working Group on Comets (Kresák), and the Director of the Central Bureau for Astronomical Telegrams (Marsden, chm). The committee is to study such matters as designations for comets discovered on old plates and for the comets found recently in the data from the SOLWIND satellite coronagraph, as well as the problem that would arise should more then 26 comet discoveries/recoveries occur within one year. The committee was also asked to prepare and publish a list of comets that received only provisional designations, including those originally identified as minor planets. A straw vote of Commission members concerning designations for comets discovered on old plates showed a strong preference for the year to be the one in which the plate was taken, rather than the one in which the discovery occurred.

The matter of the policy for review of minor planet names was addressed next, approval being given to the following recommendation:

\begin{abstract}
"All names proposed for minor planets will be reviewed for suitability, even when names are proposed by discoverers. The review will be done as indicated in the 1979 Commission 20 resolution, except that in the case of a name proposed by the discoverer the six-month waiting period for a newly numbered object can be reduced to two months. Names shall be limited to a maximum length of sixteen characters, including spaces and hyphens." [The review committee is composed of the President and Vice President of the Commission and the Director of the Minor Planet center.]
\end{abstract}

Attention was then given to the report of the ad hoc Committee on Minor Planet Program Definition [E Bowell, J Kovalevsky (chm), I K Kristensen, B G Marsden, J Schubart, V A Shor, G E Taylor, C J van Houten, J G Williams]. The revised report was read in its entirely so that all members of the Commission could be aware of recent changes. Following further discussion, the report and recommendations of the committee were adopted by the Commission as follows:

\title{
Programs of Observations of Minor Planets
}

"The ad hoc committee on programs of observations of minor planets set up by commission 20 wishes, first, to stress the importance of minor planet observations in understanding the structure, the formation, and the evolution of the solar system. An improvement of the general statistical description of the minor planet belt, of its families, and of its marginal membership (objects with orbits of high inclination or high eccentricity, Mars and Earth crossers, Trojans, Chiron or Hidalgo type planets, inactive cometary nuclei, etc.) provides clues to the dynamical evolution of the solar system and possibly to its formation. It is also a key to the understanding of the relation between various populations of the solar system (small bodies, comets, satellites and cratering processes).

"Accurate observations of particular minor planets have a major impact 
on the knowledge of the physics of these very poorly known objects (masses, possible existence of satellites, planning of eventual spacecraft missions). Celestial mechanics greatly benefits from the refined analysis of the motion of some selected minor planets and from accurate statistical descriptions of minor planet families.

"Finally, some selected minor planets are useful for determining the inertial celestial reference frame; these bodies have to be observed very accurately.

"The committee requests that Commission 20 maintain and periodically update lists of minor planets or classes of objects to be observed or searched for with given accuracy. A first approximation to these is given in the appendix.

"As a general remark, the ad hoc committee wishes to propose the following guidelines to observers:

1. Imprecise 'quick look' observations are not useful except to point out in real time the position of a newly discovered unusual object. All published observations made in the course of a given program should be reduced. Quick look observations of common objects are discouraged.

2. Most numbered planets have good ephemerides that are sufficient for many years in advance. Those with unreliable orbits should be identified in 'Efemeridy Malykh Planet'. Two or three observations of each planet every five years is all that is needed to maintain well determined orbits.

3. Whenever maximum accuracy is needed, the best available star catalog should be used. The name of the catalog should be given so that in further studies corrections for systematic catalog errors can be introduced. Other factors that limit precision should be noted. All observations should be carried out, reduced, and published in accordance with the instructions specified for each program.

4. Whenever an observation is published, observers are requested not to give more than one decimal figure beyond the accuracy of the observation.

"Finally, the committee is quite aware of the fact that it has not been able to collect information on all existing programs, so that the omission of any program is not to be considered as a sign of disapproval. On the contrary, it would like to encourage well planned new programs and requests that Commission 20 collect information to improve the lists."

\section{Appendix: Lists and classes of minor plenets recommended for observation}

1. Objects currently under study for dynamical purposes and for which the most precise positions are needed. Random errors should be better than 0.5 , but systematic errors due to instrumental or atmospheris dispersion should be much less. The observations should be referred to a well defined system (e.g., AGK3) and plate constants or dependences must be stated. Long and uniform series of at least 25 observations made by several observatories are necessary at every opposition and should extend as far as possible toward quadrature unless otherwise specified in the program instructions. The following programs belong to this class:

- The 20 selected Leningrad objects. 
- Branham's objects.

- (51) Nemausa.

- Objects making close approaches to larger planets, whose masses might thereby be determined, including (197), (348), (486), (526), (1023), (1825).

- Objects to be observed in connection with HIPPARCOS (See Scholl's presentation, above.)

2. Objects currently eligible for occultation predictions. Four or five observations per opposition, well distributed in time and extending through quadrature, are requested with an accuracy of $1 "$.

(Consultations are in progress about the list of objects.)

3. Numbered objects of general dynamical interest, for which a few observations to an accuracy of $\sim 2^{\prime \prime}$, or better if possible, are required. Three or four such observations per opposition are sufficient. Examples are:

- Trojans.

- Mars crossers.

- Minor planets beyond the Hecuba gap.

- The $3 / 1,2 / 1$ and $(N+1) / N$ librators.

- High inclination $\left(i>30^{\circ}\right.$ ) and/or high eccentricity $(e>0.4)$ objects.

- Hungarias and Phocaeas.

4. Numbered objects that need an improvement in their ephemerides. Four or five observations per opposition with an accuracy of 2 " are necessary.

(Consultations are in progress about compilation and publication of a list of such objects.)

5. Unnumbered minor planets with reasonably reliable ephemerides. Four or five observations per opposition with an accuracy of 2 " are necessary until they are numbered. Such objects should be identified and ephemerides provided in the MPCs.

6. Search for new asteroids. For each type of body, the aim should be for statistically significant sampling, in particular, for completeness to a given magnitude or in a given region. Any discovered object should be followed up by several observations with an accuracy of $2^{\prime \prime}$ during each of the two succeeding months, so as to provide a basis for good ephemerides for the next oppositions. If only single observations are obtained, the daily motion should be given to facilitate identification. The magnitude also is needed as an identifying characteristic in all cases.

- Fast moving objects

- Objects with a large component of motion in declination at opposition.

- Objects with direct motion at opposition.

- Unidentified objects with typical motion at opposition and $\mathrm{m}<18$.

A committee was to be appointed by the President of the Commission to supervise completion and updating of the lists of minor planets in need of observations. [Following consultations, appointments have been made as follows: President of the Commission (Roemer, chm), Director of the Minor Planet Center (Marsden), ITA 
representative (V A Shor), L K Kristensen, E Bowell.]

The Commission then approved the following recommendation in support of further efforts to recover lost numbered minor planets:

"Commission 20 encourages the accurate measurement of positions of minor planets from the extensive plate collections such as those at the Budapest, Goethe Link, Johannesburg, Iowell, Simeis and Turku observatories. Specifically, there is a need for measurement of plates of unnumbered minor planets for which only approximate positions were previously available. Further, in order to support the current efforts to recover the few remaining lost minor planets or to make a linkage with a possible accidental rediscovery, Commission 20 encourages the early reexamination and remeasurement of all existing plates that contain or may contain inages of those lost planets and the prompt publication of the results in the Minor Planet Circulars. Commission 20 notes with appreciation that Richard M West, Garching, has volunteered to process plates of the lost numbered planets should the original observatories be unable to do so."

Noting that a large number of IAU symposia and colloquia were already under consideration for the 1982-85 triennium, Roemer invited those planning meetings of potention interest to members of the Commission, or with proposals for meetings farther in the future, to inform her.

F K Edmondson then proposed a vote of thanks to the acting president for her excellent organization in the difficult situation occasioned by the absence of the commission president. This was adopted by acclamation, and the final session of Commission 20 at the XVIIIth General Assembly of the IAU was then adjourned.

The following corrections to the report of the Commission published in Trans. IAU XVIIIA (1982) have been noted:

p. 205 The orbital periods and semimajor axes of the three Jovian satellites should read $7^{\mathrm{h}} 08 \mathrm{~m} 0,1.79 \mathrm{R}_{\mathrm{J}} ; 16 \mathrm{~h} 11 \mathrm{~m} 3,3.08 \mathrm{R}_{\mathrm{J}}$; and $7^{\mathrm{h}} 04 \mathrm{~m} 5,1.78 \mathrm{R}_{\mathrm{J}}$ for $1979 \mathrm{~J} 1,1979 \mathrm{~J} 2$ and $1979 \mathrm{~J} 3$ respectively.

p. 210 (top) for Hygeia read Hygiea 
COMMISSION 21: LIGHT OF THE NIGHT SKY (LUMIERE DU CIEL NOCTURNE)

Reprot of Meetings, 19 and 24 August 1982

PRESIDENT : H. Tanabe

SECRETARY : Ph. Iamy

19 August 1982

\section{ADMINISTRATIVE SESSION}

\section{Welcome Address}

The president expressed his pleasure to see colleagues of Commission 21 in Greece, which is after all the cradle of astronomy. He emphasized that the past triennium was marked by active international cooperation and hoped that such cooperative work will further develop in the coming triennium.

The president invited the participants to stand in silence in memory of two members deceased in the past three years : Kazimierz Kordylewski and Donald William Schuermen, after recalling their contributions to our research field.

Besides the formal meetings of the commission and Joint Discussion $V$, in which Commission 21 participates, information was given on a related informal meeting on the Halley Optical Probe Experiment (HOPE) for the Giotto Mission to Halley's Comet organized by A.C. Levasseur-Regourd. The president requested to all speakers at the meetings to speak slowly to permit better understanding by the participants from various countries.

\section{Commission Membership}

The names of officers for the triennium 1982-1985, nominated by the IAU Executive Committee on proposal by the current officers are given :

President : R.H. Giese

Vice-President : K. Mattila

The president summarized the interests of the new president in zodiacal light and space observations, and the new vice-president in diffuse galactic and extragalactic light.

The list of the new organizing committee was accepted by the commission members attending the session, and was transmitted for approval by the Executive Committee.

Organizing Committee : R. Dumont, Yu.I. Galperin, M.S. Hanner, A.C. LevasseurRegourd, F. Srnchez-Martinez, H. Tanabe, J.L. Weinberg

The following existing IAU members were co-opted for Commission 21 membership : G.S. Ivanov-kholodny, C.F. Lillie, V.B. Nikonov, L.E. Pasinetti, C. Sánchez-Magro. Some of the names proposed by their National Committee were unknown to the members attending the session. After a discussion on this question, they were accepted.

Admissions of the following applicants to Commission 21 membership were accepted under the condition that they are admitted into the Union : P. Alvarez, W. Hofman, T. Nishimura, H.W. Ripken, M. Schissler, R.H. Zerull. 
Three names were deleted from Commission $2 l$ membership : K. Kordylewski ${ }^{\dagger}$, Th. Schmidt, D.W. Schuermant.

A new list of 27 consultants was approved for the next triennium.

\section{Reports on Astronomy 1982}

The president thanked the members for sending the information on their activities and acknowledged the help provided by R. Dumont, R.H. Giese and K. Mattila in preparation of the report. The 8-page limitation did not allow the president to include all the information he would have liked and many of airglow studies had to be excluded.

F.E. Rosch pointed out that airglow is now part of the ionospheric sciences for which Commission 21 is not concerned. In a general discussion, it was recognized that the domain of competence of Commission 21 is evolving. An opinion was expressed that the way of the commission may have to be reconsidered in the coming decade.

At a suggestion of K. Mattila, president-elect R.H. Giese expressed that he will nominate, in advance, the persons to share writing the next triennial report to maintain its high quality.

\section{The Newsletter of the Commission}

The president issued three newsletters; Nos. 4, 5 and 6, during this triennium. Newsletter No. 4 was devoted to the report of the commission meetings at Montreal General Assembly, No. 5 was a compilation of laboratory reports from each member, and No. 6 included the triennial commission report and its full references.

Warm approval and satisfaction were expressed by the members attending the session for the excellent work performed by the president.

It was agreed that one newsletter per year is adequate.

\section{Relationship with other Commissions}

The president recalled the participation of Commission 21 together with Commission 22 to the IAU Symposium No. 90 "Solid Particles in the Solar System" held in Ottawa in 1979.

In 1980, the president responded questions on airglow problems asked by Commission 50 (Protection of Existing and Potential Observatory Sites).

Commission 21 participates in Joint Discussion V "Origin and Evolution of Interplanetary Objects" to be held during this General Assembly, together with Commissions 15,20 and 22 .

\section{Other Topics}

H.W. Ripken pointed out the lack of information on the detailed programs of the scientific meetings of all commissions. After a discussion, it was agreed that the incoming president will write a letter to the General Secretary on this problem.

The administrative session came to an end with the president expressing his thanks to his vice-president, the organizing committee, the members and consultants of Commission 21 for their kind cooperation during this triennium.

The incoming president R.H. Giese, on behalf of all members, thanked very warmly the retiring president $H$. Tanabe. 
K. Mattila : Recent research on the galactic and extragalactic components of the light of the night sky (invited review paper). - An abstract of the paper has been published in Reports on Astronomy 1982 (Comission 21).

R.H. Giese : The space observation of the zodiacal light (invited review paper). - Available are more than 30 observations from 1 AU (rockets, satellites, manned spacecraft) but only few (Pioneer 10/11, Helios 1/2) from spaceprobes near the ecliptic. These, together with ground-based observations, yield as a (good but only) first approximation an ellipsoidal (or fan like) distribution of dust number density $n(r)$ with solar distance and a power law $n n r^{-1 \cdot 3}$ in ecliptic. The volume scattering function of the zodiacal light particles ( 10 to $150 \mu \mathrm{m}$ ) is compatible with recent laser scattering measurements of irregular, fluffy and meteoritic particles. To gain more insight (bimodal dust population, changes of physical properties etc.) an out-of-ecliptic photopolarimeter was $70 \%$ completed but cannot be flown due to cancellation of the NASA spacecraft for International Solar Polar Mission Space observations in the near future should concentrate to UV $(\lambda<3000 \AA)$ surveys from $1 \mathrm{AU}$ and, even more important, to investigations of the relatively unobserved regions close $\left(\lesssim 20 R_{\odot}\right.$ ) to the sun, where strong interactions of dust with solar radiation and solar wind take place.

S. Koutchmy, Ph. Lamy, G. Nikolsky : Preliminary results from the PCN experiment aboard Saliout 7. - The "Photographie du Ciel Nocturne" experiment consists in a modified Nikon camera equipped with a $58 \mathrm{~mm}$ objective at F/1.2. Color photographs taken with high speed Kodak Ektachrome film (400 ASA) were obtained in June 1982. They include : (1) the E-layer, stratified with the usual oxygen (green line) layer above an orange layer probably composed of sodium, observed for the first time; (2) noctilucent clouds photographed from space for the first time; (3) the zodiacal light between Mercury and Venus. A preliminary analysis of this latter photograph confirms that the plane of symmetry is close to the orbital plane of Venus and reveals a flatter zodiacal cloud than presently expected.

R. Robley : Does the sun disturb the zodiacal cloud? - About the position of the plane of symmetry of the zodiacal cloud the discrepancy obtained by ground-based observations has not been cancelled by the space observations : the resilts from Helios 1 and 2 are different from these of the D2A satellite. Examining the Gegenschein observations made by Tanabe (1965), it appears that the Gegenschein centre relatively to the ecliptic plane has not the same position in 1957 (solar activity maximal) as in 1962. It is proposed to explain this effect by the transverse, relatively to the ecliptic, component of the solar wind ejected through the polar coronal hole; at the opposite of the protons ejected radially (Misconi, 1976) the transverse compoment might, under certain circumtances, disturb the orbital plane of the dust grains.

24 August 1982

\section{SCIENTIFIC SESSION}

H.W. Ripken : Interaction processes between the solar wind and zodiacal dust particles, and relevant observational consequences. - Solar wind ions impinge on zodiacal dust grain surfaces, penetrate, neutralize, and desorb from saturated surfaces into free space. Before ionization occurs the neutral particles resonantly scatters solar radiation. To model this radiation and, by comparisons with observations, gain information on dust-desorbed neutrals and dust grains, the neutral particle density and the velocity distribution function have to be computed. Methods and results are presented. Resonance radiation calculations for $H$ and He yield integral and spectral EUV intensities and provide information on the integral dust cross section, spatial and temporal depsity pradients, the velocity distribution 
function, and particle erosion rates.

J. de Buitrago, F. Sánchez-Martinez : Dynamics of zodiacal dust and new inversion techniques. - The zodiacal cloud, is considered as a system essentially ruled by gravitation and radiation forces acting as a perturbation. Within this context, it is found that spatial distribution of the kind $n\left(r, \beta_{\odot}\right) \sim r^{-v} \cdot f\left(\beta_{\odot}\right),(\nu>1)$, are adequate and consistent. In this picture, the angular distribution $f\left(\beta_{\odot}\right)$, is not determined by the dynamics, remaining mostly dependent upon the injection mechanism. If we accept $\nu=1$ as an approximation, it can be shown that for observations in the helioecliptic plane, the Brightness Integral can be formally transformed into a second order integral equation, with $f\left(\beta_{\odot}\right)$ as the incognite function, which can be solved numerically. Figures of $f\left(B_{\odot}\right)$ and the obtained zodiacal cloud profile are presented.

Ph. Lamy, J.M. Perrin : A new model of light scattering by Large irregular grains. - This new model combines the eikonal model for the diffraction part and geometrical optics for the scattering at large angles (as developed by $M$. Wolff for planetary regolith). Progress has been made by obtaining the exact expression of the eikonal function. The experimental results obtained by $\mathrm{K}$. Weiss by laser scattering of irregular grains are well reproduced both in intensity and polarization, in particular the broadening of the diffraction lobe.

Ph. Lamy : On the bulk density of interplanetary dust grains. - The sources of all possible information on the density of interplanetary grains are reviewed. It is shown that the sources leading to small values (i.e. $<1 \mathrm{~g} \mathrm{~cm}^{-3}$ ) are either indirect or unreliable (meteors, space experiments, early dust collections). On the contrary, reliable sources, such as the extraterrestrial grains collected by Brownlee and the analysis of the lunar microcraters, indicate "normal" densities, typically $2 \mathrm{~g} \mathrm{~cm}^{-3}$, definitely larger than $1 \mathrm{~g} \mathrm{~cm}^{-3}$.

R. Dumont : Recent progress of the inversion technique in zodiacal light interpretation (invited review paper - read by A.C. Levasseur-Regourd). - An increasing amount of works have been made during this triennium towards an inversion of the brightness integral or towards some localization of the contributions along the line-of-sight. A main distinction is between the "local inversion" (where the instrument is located) and the "extended inversion". Practical results of recent works are the shape of the phase function (moderate forward enhancement) and the increase with heliocentric distance of the local polarization degree at $90^{\circ}$ scattering angle.

A.C. Levasseur-Regourd : P.I.R.A.M.I.G./Saliout 7 comera - Photographic high sensitivity comera for visible and near Infra-Red light, studying Atmosphere, Interplanetary Medium and Galaxies. - P.I.R. $\bar{A} . M . I . \bar{G}$. has been develope $\bar{d}$ at Laboratoire d'Astronomie spatiale (IAS) and is used by a team of scientists of Service d'Aéronomie LAS and Institut d'Astrophysique in France and of USSR. It is devoted to the detection of large faint sources and discriminates between atmospheric and interplanetary/ galactic sources through orientation of the orbital station. During the French "Spationaut" mission in June 1982, more than 300 pictures (including sensitometry and duration) were taken. Preliminary results show the spatial extension of $\mathrm{OH}$ waves, the vertical structure of emissive layers, and absorbing regions close to galactic center. Results about zodiacal light polarization in infra-red, symmetry surface, Gegenschein and libration points may be expected.

P.V. Kulkarni : Low Latitude airglow (invited review paper) - With the new interest in the $F$ region of the atmosphere where detection of depletions and irregularities has become important, airglow in the $F$ region is gaining importance. Column emission rate of $1356 \AA$ or $7774 \AA$ of oxygen gives the peak electron density while addition of $6300 \AA$ measurement gives the height of maximum electron density of the $\mathrm{F}$ region. With high spatial resolution $(\sim 0.1)$ at an appropriate site $\left( \pm 18^{\circ} \pm 5^{\circ}\right.$ magnetic latitude) it is possible to map the "bubbles" and "plumes" in the tropical 
F region. It was shown that by measuring a line and a branch emission of an $O H$ band, temperature of the mesospheric region can be monitored and the periodicities found could be connected to the gravity waves in the atmosphere.

P. Alvarez, F. Sanchez-Martinez : Spectmum of the atmospheric continuum. During 1978 and 1979 the spectrum (4000 \& - 8500 \&) of the light of the night sky in the celestial north pole direction has been monitored at Izaña (Tenerife). These measurements show a well correlated ( 0.75$)$ atmospheric continuum emission between $4000 \AA$ and $7000 \AA$, but not correlated with the same emission at $8200 \AA$. This fact has enabled us to determine the profile of this emission between $4000 \AA$ and $7000 \AA$, making no assumptions on the extraterrestrial components. It shows a broad maximum at $5500 \AA$, bluer than the obtained in 1972 by Sternberg and Ingham. The zenithal mean absolute emission of the atmospheric continuum obtained at $5500 \AA$ is $0.25 \mathrm{R} / \AA$ and at $8200 \AA$ is about $0.35 \mathrm{R} / \AA$.

A.S. Asaad : Recent observation of zodiacal Zight in Egypt. - Results of zodiacal light observation at Abu-Simbel since 1975 show an increase of brightness with decreasing solar activity in different colours. Colour indices $B-V, B-R$ increase with sun's elongation during quiet solar activity while colour index $B-V$ decreases during high activity and no variation is noted in between. Lunar variation of zodiacal light is noted, showing a peak at new moon. Such variations are not yet observed from outer space. If however sufficiently accurate and long period observations from outer space are obtained, then the variations noted by many observers may be attributed to the Earth's atmosphere. Lunar variations can be explained on this basis.

H. Tanabe, A. Takechi, A. Miyashita: An interpretation of the false zodiacal iight. - The false zodiacal light has been observed by several zodiacal light observers since the beginning of the last century, but its full explanation is not found yet. From our photographic and spectroscopic observations made in 1978 and from descriptions of previous observers, we have reached the conclusion that the false zodiacal light may be a real zodiacal band seen wider than usual due to the combination of the luminance difference threshold of the human eye and a special distribution of the airglow ( $5577 \AA$ ) in the sky : namely, that the airglow be faint in the region of faint astronomical light.

F.E. Roach : Overview of a half century of the light of the night sky. - No abstract available. 
Report of Meetings, 18 and 24 August 1982 .

PRESIDENT: W.G. E1ford

SECRETARI ES: B.A. McIntosh

B.A. Lindblad

\section{August 1982}

The President welcomed 14 members and 2 consultants. Apologies were received from L. Kresak, B.A. Lindblad and Z. Sekanina.

\section{OFFTCERS AND MEMBERSHTP}

The following nominations for officers of the Commission were endorsed : President, O.I. Belkovich; Vice President, D.E. Brownlee; Organizing Committee: P.B. Babadzhanov, W.J. Baggaley, W.G. Elford, H. Fechtig, M.S. Hanner, J.A.M. McDonnel1, J. Stohl, K. Tomita.

The President announced the names of six new members: A.T. Blackwe11, E.Grun, M. Hajdukova, P. Lamy, H. Miles, H.W. Ripken. Four other nominations were held over until the next business meeting pending confirmation of membership. Consultants appointed for the next term are: G. Cevolani, K.B. Hindley, F. Horz, J.W. Mason, T. Nasagawa, G. Schwehm, J. Trulsen, D.K. Yeomans.

The President reported the deaths of three members of the Commission since the last meeting: V. Guth, H. Hirose, D.W. Schuerman.

II REPORT OF THE COMMISSION

The published report to be considered at the next business meeting. An extended report was tabled.

\section{IIL PROJECTS AND WORKING GROUPS.}

1. International Halley Watch

Dr I. Halliday reported on the work of the Steering Commitcee for the International Halley Watch. While there is no separate discipline for the study of meteors associated with Comet Halley it was agreed that Commission 22 should be responsible for encouraging regular observation of the Orionid and $n$-Aquarid showers during the next 5-10 years.

It was pointed out that although there may be no dramatic change in the observed fluxes, studies of the structure of these streams during the Halley apparition are very important in complementing the comet observations. Dr Millman reported that he is preparing a paper summarizing the present state of knowledge concerning meteor showers associated with comet Halley, and he agreed to distribute reprints to nembers of Commission 22 .

It was noted that Dr Z. Sekanina of J.P.L. (USA) is the archivist for meteor data relating to the Halley apparition. A resolution on $\mathrm{IHW}$ was deferred unt1 the next meeting. 


\section{Project GLOBMET}

The President outlined the background to GLOBMET (Global Meteor Observation System) which had been proposed by the Soviet Geophysical Committee and supported by resolutions at the IAMAP meeting in Hamburg (August, 1981) and the IAGA meeting at Edinburgh (August, 1981). A committee had been formed within SCOSTEP with representatives from IAGA, IAMAP, IAU and URSI to produce a GLOBMET planning document. A draft document was now before Commission 22 for comment, with the aim of having a document in final form to present to the IUGG Assembly in August 1983.

The main objective of the project is to promote a network of radio meteor observatories to study: (1) the atmospheric circulation in the meteor region, (2) the influx and distribution of meteors in the neighbourhood of the earth, (3) models of meteor/atmosphere interaction.

Several speakers pointed out that the data sought by workers concerned with meteor wind studies were not always suitable for astronomical studies. It was agreed that a working group, (C.S.L. Keay, O.I. Belkovich, B.A. McIntosh and J. Stoh1) examine the proposed astronomical objectives and bring a recommendation to the next meeting.

\section{European and Canadian Fireball Networks}

The meeting noted with concern the decision to close down two stations of the European network that are operated by the University Observatory of the Viennese Institute, and also the doubt about confirmed financial support for the MORP network (Canada). It was agreed that a working group (Z. Ceplecha, I. Halliday, C.S.L. Keay) prepare resolutions of support for these networks for presentation to the next meeting.

\section{August 1982}

The meeting was attended by 20 members, 2 consultants and 3 observers. Apologies were received from R.H. Giese, P. Lamy and Z. Sekanina.

I MEMBERSHIP

In addition to the six new members noted at the previous meeting, the following were recorded as new members: I. Hasegawa, R.L. Newburn, E.F. Tedesco, A.K. Terenteva. The name of I.P. Williams was added to the list of consultants. The total membership was now 87 plus 10 consultants.

\section{REPORT OF THE COMMI SSION}

The President tabled the draft report that is to appear in the Transactions, and expressed his thanks to those who had prepared the various sections of the report. Due to space constraints in the Transactions the printed report will be a significant abbreviation of the material submitted. The full report will be posted to all members. The draft report was approved without discussion.

\section{RESOLUTIONS}

Three working groups presented the following resolutions which were adopted:

1. Resolutions concerning the European and Canadian Fireball Networks

Noting the importance of the recent contributions from the Canadian and European Fireball Networks, Commission 22

(a) recommends that continued support be provided for these observational programs in order that a more extensive body of rellable data may be gathered for critical analysis. Although the recovery of meteorites from these two 
major camera networks is likely to remain limited to rare events, progress in the interpretation of fireball data is now leading to significant studies of both the astronomical properties of meteorites and the physics of their atmospheric interaction.

(b) recommends that the University Observatory of Vienna should give its fullest support to the continuation of operation of the two Austrian stations of the European Fireball Network because of their key position in the Network and their essential role in the location and recovery of meteorites which fall on Austrian soil.

\section{Resolution concerning International Halley Watch}

Noting the major effort which will be made to observe Comet Halley, organized by the International Halley Watch, Commission 22

recommends that meteor astronomers pay particular attention to meteor showers assoclated with the comet, the Orionid and Eta-Aquarid showers, since a better understanding of the meterolds from the comet can contribute to an increased knowledge of the comet itself.

\section{Resolutions concerning project GLOBMET}

Noting the request from SCOSTEP for comments on the GLOBMET document, and recognizing that data from GLOBMET can be of astronomical importance, Commission 22

recommends that,

(1) the GLOBMET document emphasize that the data requirements for astronomical purposes may differ from those for geophysical purposes,

(2) the Manual on Meteor Radar Observations include a section on Parameters of Astronomical Importance,

(3) a list of the parameters of astronomical importance prepared by the Working Party be sent to the convenors of the GLOBMET project.

IV . PROJECTS

\section{Meteor Data Centre, Lund, Sweden}

The President reported that a Meteor Data Centre had been set up by Dr B.A. I. Indblad at the Lund Observatory in Sweden, and that the Executive Committee had allocated SwFr 1800 per annum for the three calendar years 1983-85 towards the cost of maintaining the Centre.

Dr Lindblad stated that the purpose of the Meteor Data Centre is to collect and store data obtained by photographic and radar methods on atmospheric trajectories and heliocentric orbits of meteors and fireballs. Limited amounts of data relating to other meteor programs such as radar-visual studies, meteor radar rates etc., are also avallable. Computer programs for stream searching and listing will also be avallable.

Orbital and trajectory data have been obtained mainly from published sources. There is also a limited amount of unpublished data. Data can be supplied on IBM cards, tape or as a computer listing. Duplicate copies of the photographic meteor data are also avallable on request from Dr $Z$. Ceplecha, Ondre jov Observatory, Czechos lovakia.

\section{$\checkmark$ GENERAL BUSINESS}

1. Circulation of Agenda of Business and Scientific Meetings

Dr Ripken polnted out that the Agenda of the Business and Scientific meetings 
had only become available a day or two before each meeting. He suggested that such material should be supplied to the Secretariat prior to the General Assembly so that the information could be made available to all participants, possibly by inclusion in the reglstration material. It was agreed that the president should consult with the General Secretary on this matter.

\section{Appreciation}

The President thanked those present for their participation. The incoming President expressed the members' thanks to the retiring President for his work on behalf of the Commission during the past three years.

VI. SCIENTIFIC PRESENTATIONS

Eleven short contributions were presented during this session.

I.P. Williams :

0.I. Belkovich, Sulejmanov and Tokhtasjev :

K.B. Hindley :

H. Miyashita and H. Tanabe :

A. Hajduk and G. Cevolani :

J.W. Mason :

R.L . Hawkes, J. Jones and

Z. Ceplecha:

P. Pecina and Z. Ceplecha :

B.A. McIntosh :

C.S.L. Keay :

J. Stoh1 :
The evolution of the Quadrantid and Gemintd meteor streams.

Structure of the Gemintd and Quadrantid meteor streams.

Recent studies of the Quadrantid and Taurid meteor streams.

Recent activity of meteor showers observed at the Tokyo Astronomical Observatory.

Zones of increased particle flux density around the orbit of Comet Halley .

Recent studies of the Perseld meteor streams.

The height and orbital distributions of TV meteors.

New aspects in single body meteor physics. Infrasound from meteors : a negative result. A microprocessor radar meteor echo analyser. On the distribution of sporadic meteors. 
$\underline{\text { Report of Meetings on } 18,19 \text { and } 23 \text { August } 1982 .}$

PRESIDENT: H. Eichhorn

SECRETARY: J. L. Russell

Most of the business aspects of the Commission were conducted during the business meeting on 18 August. President Eichhorn welcomed the members of the commission and asked them to stand in memory of those colleagues who have died since the last meeting: Johannes Haas, Paul Herget and Jan Schilt, as well as of Valfrids Oswaldo who was not a member of our Commission at the time of his death.

The President then announced the appointment of Jane L. Russell as Secretary of the Commission. As a result of the elections and carry over, the following will be the Officers and the Organizing Committee for the trienuium 1982-1985:

President: Wilhelm Gliese

Vice President: Arthur R. Upgren

Organizing Committee: T. E. Corbin, H. Eichhorn. G. D. Gatewood, I. I. Kanaev, T. E. Lutz, J. D. Stock, W. F. van Altena.

The following were unanimously approved as new members of the Commission:

A. N. Argue, R. Branham, Liang-tia G. Chiu, J. Fanselow, F. Firneis, A. Fresneau, P. D. Hemenway, J. Hughes, W. H. Jefferys, K. Johnston, L. Stange, J. W. Stein, R. C. Stone, C. Turon, H. G. Walter, G. Westerhout. Standards:

The Commission approved the following report of the Working Group on Parallax

\section{REPORT OF THE WORKING GROUP ON PARALLAX STANDARDS \\ TO I.A.U. COMMISSION 24}

\section{A. R. Upgren, Chairman}

The Working Group on Parallax Standards was established at the meeting of Commission 24 during the XVII General Assembly of the International Astronomical Union in Montreal in August 1979. Its purpose is to investigate and propose methods of evaluating and reducing systematic parallax errors arising between observatories. Its concentration has been on the establishment of a number of star fields which would serve as standards observed by all parallax programs in the usual manner in which they obtain parallax observations.

Two closely related objectives emerged in the course of the group's deliberations. The first of these is the necessity for continued monitoring and calibration of each telescope and the second is the evaluation of parallax differences between observatories. Fields centered on open clusters are best suited for the first objective since many more stars are available for measure than the terms necessary for modeling each of the telescopes.

The Working Group recommends that the first of these objectives be met by regular evening and morning parallax observations of three fields each centered on an open cluster. The three fields are roughly equally separated in right ascension and are accessible to observatories at all latitudes. The fields are: 


$\begin{array}{lll}\text { Object } & \text { R.A.1980 Dec. } & \text { Object at Center } \\ \text { Pleiades } & 3^{\mathrm{h}_{4} 5^{\mathrm{m}} \cdot 1+24^{\circ} 08^{\prime}} & \mathrm{BD}+23^{\circ} 520 \\ \text { Praesepe } & 839.2+1945 & \mathrm{BD}+23^{\circ} 2166\end{array}$

In selecting a larger list of individual stars and star fields, the Working Group sought to optimize the following features: widespread and uncorrelated distributions in magnitude, color, right ascension and declination. To a lesser extent, the absence of detectable orbital motion in the target star or stars, availability of past parallax observations, minimal disruption of presently active parallax programs and astrophysical interest of the target star or stars were also taken into consideration. A preliminary examination of each field has been made in order to be sure that an adequate distribution of suitable reference stars is available for measure.

In order to accomodate all programs, the list of stars has been divided into three sections. The first section contains those stars which it is hoped will be observed on all programs and includes mostly stars already being observed by a majority of them. The second section is a supplementry list intended to increse the number of standards for the programs which can accomodate a larger number. Both sections are limited to target stars between apparent magnitudes 7 and 14 because this is the magnitude interval accessible to most active parallax telescopes with a need for magnitude reduction devices which is small compared to that for stars covering a wide range in apparent magnitude. The third section includes brighter stars, (magnitude 6 and brighter) most of which are among the brightest and closest to their spectral type, along with a smaller number of very bright but distant stars whose true parallax is known in advance to be near zero. This list is proposed with the awareness that ground based telescopes and future space satellites with astrometric capability may both be helpful in establishing a zero point for the system, free of systematic effects at all magnitudes. It also focuses the continued need for parallax programs to respond to problems of stellar astrophysics insofar as they are able to do so, and in the course of calibration, to provide the astronomical community with at least a few highly precise parallaxes of many kinds of stars. Four of the brightest and nearest stars do not appear on this list. They are Sirius, Procyon, Altair and Alpha Centauri. They have parallaxes presently known with one or two percent error and since all four are binaries with considerable orbital motion, the possible uncertainty in their orbital elements makes further improvement in their parallaxes doubtful and not worth the effort involved in their redetermination.

The stars are listed in three tables numbered as indicated above. Each table lists one or more identifications of the central or target star. For all stars, an identification chart is available. Positions for the central star or stars are given for both 1950 and 2000 and $B$ and V magnitudes are both given for ease of use by those with either photographic or visual refractors.

The Working Group recommends a periodic review of the observations of each participating observatory or satellite program, including measures with an automatic machine as necessary. An initial evaluation of the observations, including a critical examination of the available reference star frames, should be made after about three to five years. This plan should be modified as needed after an evaluation is made at that time.

We urge all observatories active in parallax observations to extend their programs to include the three cluster fields, the primary standards listed in Table I and as many of the secondary and bright star fields as possible. 
Table I

Primary List of Parallax Standard Fields

\begin{tabular}{|c|c|c|c|c|c|c|c|c|c|c|}
\hline \multirow[b]{2}{*}{ Field } & \multirow[b]{2}{*}{ Principal Star } & \multicolumn{3}{|c|}{1950} & \multicolumn{3}{|c|}{2000} & \multirow[b]{2}{*}{$\mathrm{V}$} & \multirow[b]{2}{*}{ B } & \multirow[b]{2}{*}{$\underline{B}-\mathrm{V}$} \\
\hline & & & R.A. & Dec. & & R.A. & Dec. & & & \\
\hline 1. & $\mathrm{HD} 225213$ & & $\mathrm{~h}_{02.5}$ & $-37^{\mathrm{o}} 36^{\prime}$ & & 05.2 & $-37^{\circ} 19^{\prime}$ & 8.63 & 10.08 & 1.45 \\
\hline 2. & van Maanen $2=\mathrm{G} 135$ & 0 & 46.5 & +509 & 0 & 49.2 & +525 & 12.37 & 12.93 & 0.56 \\
\hline 3. & $\begin{array}{l}\mathrm{BD}+14^{\circ} 699=\text { vA } 459 \\
\mathrm{BD}+13^{\circ} 685=\text { vA } 472\end{array}$ & & $\begin{array}{l}25.2 \\
25.3\end{array}$ & $\begin{array}{l}+1346 \\
+14 \quad 10\end{array}$ & $\begin{array}{l}4 \\
4\end{array}$ & $\begin{array}{l}28.1 \\
28.2\end{array}$ & $\begin{array}{ll}+13 & 52 \\
+1416\end{array}$ & $\begin{array}{l}9.52 \\
9.03\end{array}$ & $\begin{array}{r}10.45 \\
9.87\end{array}$ & $\begin{array}{l}0.93 \\
0.84\end{array}$ \\
\hline 4. & HD $33793=$ Kapteyn's Star & 5 & 09.7 & -4500 & 5 & 11.4 & -4456 & 8.81 & 10.37 & 1.56 \\
\hline 5. & $\mathrm{BD}-3^{\circ} 1123$ & 5 & 28.5 & -343 & 5 & 31.2 & -340 & 7.97 & 9.44 & 1.47 \\
\hline 6. & G87-7 = G1 246 & 6 & 44.2 & +3735 & 6 & 47.4 & +3731 & 12.10 & 12.02 & -0.08 \\
\hline 7. & $\mathrm{BD}-20^{\circ} 2665$ & 8 & 42.5 & $-21 \quad 16$ & 8 & 45.0 & -2127 & 10.16 & 11.38 & 1.22 \\
\hline 8. & Ross $434=$ G1 366 & 9 & 41.7 & $+76 \quad 17$ & 9 & 47.1 & $+76 \quad 03$ & 10.63 & 12.06 & 1.43 \\
\hline 9. & HD $95735=$ Lal 21185 & 11 & 00.6 & +3618 & 11 & 03.5 & +3601 & 7.50 & 9.00 & 1.50 \\
\hline 10 . & Ross $128=\mathrm{G} 1447$ & 11 & 45.1 & +1.06 & 11 & 47.4 & +049 & 11.10 & 12.86 & 1.76 \\
\hline 11. & BPM 36430 & 11 & 53.7 & -4824 & 11 & 56.4 & $-48 \quad 40$ & 12.85 & 12.65 & -0.20 \\
\hline 12. & $\mathrm{BPM}+2^{\circ} 2538=$ Ross 456 & 12 & 23.0 & +134 & 12 & 25.3 & +117 & 9.61 & 10.06 & 0.45 \\
\hline 13. & $\begin{array}{l}\text { Ross } 476=\text { Wolf } 485 \mathrm{~B} \\
\mathrm{BD}-7^{\circ} 3632=\text { Wolf } 485 \mathrm{~A}\end{array}$ & & $\begin{array}{l}27.5 \\
27.7\end{array}$ & $\begin{array}{l}-827 \\
-819\end{array}$ & $\begin{array}{l}13 \\
13\end{array}$ & $\begin{array}{l}30.2 \\
30.5\end{array}$ & $\begin{array}{l}-842 \\
-834\end{array}$ & $\begin{array}{l}14.34 \\
12.31\end{array}$ & $\begin{array}{l}15.97 \\
12.38\end{array}$ & $\begin{array}{l}1.63 \\
0.07\end{array}$ \\
\hline 14. & Proxima Cen $=$ G1 551 & 14 & 26.3 & -6228 & 14 & 30.2 & -6241 & 11.05 & 13.02 & 1.97 \\
\hline 15. & $\mathrm{G} 202-48=\mathrm{G} 1625$ & 16 & 24.2 & +5425 & 16 & 25.2 & +54.18 & 10.14 & 11.75 & 1.61 \\
\hline 16. & G240-72 & 17 & 78.9 & +7053 & 17 & 52.0 & +7052 & 14.15 & 14.55 & 0.40 \\
\hline 17. & Barnard's Star = G1 699 & 17 & 55.4 & +433 & 17 & 58.1 & +432 & 9.54 & 11.28 & 1.74 \\
\hline 18. & Wolf 940 & 21 & 44.1 & -024 & 21 & 46.4 & -0.10 & 12.67 & 14.29 & 1.62 \\
\hline 19. & Ross $248=\mathrm{G} 1905$ & 23 & 39.4 & +4355 & 23 & 42.1 & +4411 & 12.29 & 14.21 & 1.92 \\
\hline 20 . & $\begin{array}{l}\mathrm{G} 130-6=\mathrm{G} 1905 \cdot 2 \mathrm{~B} \\
\mathrm{G} 130-5=\mathrm{G} 19052 \mathrm{~A}\end{array}$ & $\begin{array}{l}23 \\
23\end{array}$ & $\begin{array}{l}41.3 \\
41.4\end{array}$ & $\begin{array}{ll}+32 & 16 \\
+32 & 19\end{array}$ & $\begin{array}{l}23 \\
23\end{array}$ & $\begin{array}{l}44.0 \\
44.1\end{array}$ & $\begin{array}{ll}+32 & 32 \\
+32 & 35\end{array}$ & $\begin{array}{l}12.90 \\
11.73\end{array}$ & $\begin{array}{l}13.05 \\
13.28\end{array}$ & $\begin{array}{l}0.15 \\
1.55\end{array}$ \\
\hline
\end{tabular}


Table II

Secondary List of Parallax Standard Fields

\begin{tabular}{|c|c|c|c|c|c|c|c|c|}
\hline & & & & 20 & & & & \\
\hline Field & Principal Star & R.A. & Dec. & R.A. & Dec. & V & B & $\underline{B-V}$ \\
\hline 1. & $\mathrm{CoD}-31^{\circ} 662$ & $1^{h_{30} m_{0}}$ & $-30^{\circ} 56^{\prime}$ & $1^{\mathrm{h}_{32} \mathrm{~m}_{2}}$ & $-30^{\circ} 40^{\prime}$ & 10.78 & 11.42 & 0.64 \\
\hline 2. & Wolf $110=\mathrm{G} 3-36$ & 200.1 & +528 & 202.5 & +542 & 12.33 & 13.77 & 1.44 \\
\hline 3. & $\mathrm{BD}+33^{\circ} 529=$ Wolf 1323 & 249.0 & +3412 & 252.0 & $+34 \quad 24$ & 9.55 & 10.89 & 1.34 \\
\hline 4. & Ross $578=$ G160-5 & 335.8 & -1137 & 338.4 & $-11 \quad 27$ & 13.01 & 14.69 & 1.68 \\
\hline 5. & LHS 1565 & 334.3 & $-44 \quad 40$ & 336.1 & -4430 & 13.03 & 14.93 & 1.90 \\
\hline 6. & $\begin{array}{l}\text { Stein 2051A }=\text { G1 } 169.1 \mathrm{~A} \\
\text { Stein 2051B }=\text { G1 } 169.1 \mathrm{~B}\end{array}$ & $\begin{array}{ll}4 & 26.7 \\
4 & 26.7\end{array}$ & $\begin{array}{ll}+58 & 53 \\
+58 & 53\end{array}$ & $\begin{array}{ll}4 & 31.3 \\
4 & 31.3\end{array}$ & $\begin{array}{l}+5859 \\
+5859\end{array}$ & $\begin{array}{l}11.09 \\
12.44\end{array}$ & $\begin{array}{l}12.73 \\
12.75\end{array}$ & $\begin{array}{l}1.64 \\
0.31\end{array}$ \\
\hline 7. & Ross $41=$ G1 203 & 525.3 & +937 & 528.1 & +939 & 12.48 & 14.12 & 1.64 \\
\hline 8. & Ross $986=$ G1 268 & 706.6 & +3838 & 710.2 & $+38 \quad 33$ & 11.48 & 13.19 & 1.71 \\
\hline 9. & Wolf $1421=G 1289$ & 745.2 & +2030 & 748.2 & $+20 \quad 22$ & 11.47 & 12.99 & 1.52 \\
\hline 10 . & $\mathrm{BD}+31^{\circ} 1684$ & 750.4 & $+30 \quad 46$ & 753.4 & $+30 \quad 38$ & 8.30 & 8.92 & 0.62 \\
\hline 11. & G51-15 & 826.9 & +2657 & 830.3 & +2647 & 14.81 & 16.87 & 2.06 \\
\hline 12. & $\mathrm{BD}+42^{\circ} 1922$ & 841.9 & +4152 & 845.5 & +4141 & 8.60 & .54 & 0.94 \\
\hline 13. & Ross $85=$ G1 361 & 938.5 & +1326 & 941.3 & $+13 \quad 12$ & 10.40 & 11.90 & 1.50 \\
\hline 14. & G163-27 & 1055.1 & -715 & 1057.4 & -731 & 14.33 & 14.65 & 0.32 \\
\hline 15. & $\mathrm{BD}-17^{\circ} 3526$ & 1157.6 & $\begin{array}{ll}-18 & 07\end{array}$ & 1200.3 & -1823 & 10.78 & 11.95 & 1.17 \\
\hline 16. & $\begin{array}{l}\text { HD } 134439 \\
\text { HD } 134440\end{array}$ & $\begin{array}{ll}15 & 07.2 \\
15 & 07.2\end{array}$ & $\begin{array}{ll}-16 & 13 \\
-16 & 13\end{array}$ & $\begin{array}{ll}15 & 10.0 \\
15 & 10.0\end{array}$ & $\begin{array}{ll}-16 & 24 \\
-16 & 24\end{array}$ & $\begin{array}{l}9.02 \\
9.43\end{array}$ & $\begin{array}{r}9.81 \\
10.28\end{array}$ & $\begin{array}{l}0.79 \\
0.85\end{array}$ \\
\hline 17. & Ross $1057=\mathrm{G} 257-20$ & $15 \quad 42.2$ & $+76 \quad 10$ & 1541.1 & +7603 & 12.22 & 13.87 & 1.65 \\
\hline 18. & Ross $808=\mathrm{G} 180-23$ & 1559.6 & +3657 & $16 \quad 01.5$ & +3648 & 14.36 & 14.53 & 0.17 \\
\hline 19. & $\mathrm{COD}=38^{\circ} 10980$ & 1620.2 & $\begin{array}{ll}-39 & 07\end{array}$ & $16 \quad 23.4$ & $\begin{array}{ll}-39 & 14\end{array}$ & 11.00 & 11.14 & 0.14 \\
\hline 20 . & Wolf $1346=$ G1 794 & $20 \quad 32.2$ & +2454 & $20 \quad 34.3$ & +2504 & 11.53 & 11.47 & -0.06 \\
\hline 21. & Ross $769=$ G1 817 & 2102.2 & $\begin{array}{ll}-17 & 08\end{array}$ & 2105.1 & -1656 & 11.44 & 12.88 & 1.44 \\
\hline 22 . & Wolf $1106=\mathrm{G} 231-27$ & 2106.8 & +5934 & 2108.3 & +5946 & 13.30 & 14.85 & 1.55 \\
\hline 23 . & $\mathrm{BD}+8^{\circ} 4638$ & 2114.1 & +911 & 2116.4 & +923 & 7.95 & 8.97 & 1.02 \\
\hline 24 . & $\mathrm{COD}-49^{\circ} 13515$ & 2130.2 & $\begin{array}{ll}-49 & 13\end{array}$ & 2133.4 & $-48 \quad 59$ & 8.70 & 10.16 & 1.46 \\
\hline 25. & $\mathrm{G} 261-45=\mathrm{G} 1836.2$ & 2136.7 & +8249 & 2134.3 & $+83 \quad 02$ & 13.02 & 13.00 & -0.02 \\
\hline 26 . & $\mathrm{BD}+42^{\circ} 4471$ & 2238.3 & +4245 & 2240.4 & +4300 & 9.81 & 10.92 & 1.11 \\
\hline
\end{tabular}


Table III

Parallax Standards - Bright Stars

\begin{tabular}{|c|c|c|c|c|c|c|c|c|}
\hline \multirow[b]{2}{*}{ Field } & \multirow[b]{2}{*}{ Principal Star } & \multicolumn{2}{|c|}{1950} & \multicolumn{2}{|c|}{2000} & \multirow[b]{2}{*}{$\mathrm{V}$} & \multirow[b]{2}{*}{ B } & \multirow[b]{2}{*}{$\underline{B-V}$} \\
\hline & & R.A. & Dec. & R.A. & Dec. & & & \\
\hline 1. & $\beta$ Cas & $0^{\mathrm{h}_{0}} 6^{\mathrm{m}_{5}}$ & $+58^{\circ} 52^{\prime}$ & $0^{\mathrm{h}_{0} 9^{\mathrm{m}} \mathrm{2}}$ & $+59^{\circ} 09^{\prime}$ & 2.25 & 2.60 & 0.35 \\
\hline 2 . & $\alpha$ Er $\mathbf{i}$ & 135.8 & -5730 & 137.7 & $-57 \quad 15$ & 0.47 & 0.28 & -0.19 \\
\hline 3 . & $\alpha \operatorname{Per}$ & 320.8 & +4940 & 324.3 & +4951 & 1.79 & 2.27 & 0.48 \\
\hline 4. & $\varepsilon \operatorname{Er} \mathbf{i}$ & 330.5 & -938 & 332.9 & -928 & 3.73 & 4.62 & 0.89 \\
\hline 5. & $\alpha$ Tau & 433.0 & +1624 & 435.9 & +1630 & 0.86 & 2.39 & 1.53 \\
\hline 6. & $\pi^{3}$ Or i & $4 \quad 47.1$ & +652 & 449.8 & +657 & 3.19 & 3.64 & 0.45 \\
\hline 7. & $\beta$ Or $\mathrm{i}$ & $\begin{array}{ll}5 & 12.1\end{array}$ & -816 & 514.5 & -812 & 0.08 & 0.05 & -0.03 \\
\hline 8. & $\alpha$ Or i & $5 \quad 52.5$ & +724 & 555.2 & +724 & $0.80 \mathrm{v}$ & $2.66 \mathrm{v}$ & 1.86 \\
\hline 9 . & $\alpha$ Car & 622.8 & -5240 & 624.0 & $\begin{array}{ll}-52 & 41\end{array}$ & -0.73 & -0.57 & 0.16 \\
\hline 10 . & $\delta \mathrm{CMa}$ & 706.4 & -2619 & 708.4 & -2624 & 1.84 & 2.52 & 0.68 \\
\hline 11. & B Gem & 742.3 & +2808 & 745.3 & +2801 & 1.15 & 2.15 & 1.00 \\
\hline 12. & $\alpha$ Leo & $10 \quad 05.7$ & +1212 & $10 \quad 08.4$ & +1158 & 1.36 & 1.25 & -0.11 \\
\hline 13. & HD 103095 & 1150.1 & $+38 \quad 05$ & 1153.0 & +3743 & 6.45 & 7.20 & 0.75 \\
\hline 14. & $\gamma \mathrm{UMa}$ & 1151.2 & +5358 & 1153.8 & +5342 & 2.44 & 2.44 & 0.00 \\
\hline 15. & $\delta \mathrm{UMa}$ & 1213.0 & +5718 & 1215.4 & +5702 & 3.31 & 3.39 & 0.08 \\
\hline 16. & B CVn & 1231.4 & +4138 & 1233.8 & +4121 & 4.27 & 4.86 & 0.59 \\
\hline 17. & $\mathrm{~B} \mathrm{Com}$ & 1309.5 & +2808 & 1311.9 & +2753 & 4.26 & 4.84 & 0.58 \\
\hline 18 . & $n \mathrm{Boo}$ & 1352.3 & +1839 & 1354.7 & +1824 & 2.69 & 3.27 & 0.58 \\
\hline 19. & $\alpha$ Boo & $14 \quad 13.4$ & +1926 & 1415.7 & +1911 & -0.06 & 1.17 & 1.23 \\
\hline 20 & $\alpha$ Lyr & $18 \quad 35.2$ & +3844 & $13 \quad 36.9$ & $+38 \quad 47$ & 0.04 & 0.04 & 0.00 \\
\hline 21. & $\alpha$ Cyg & $\begin{array}{ll}20 & 39.7\end{array}$ & +4506 & 2041.4 & $+45 \quad 16$ & 1.26 & 1.35 & 0.09 \\
\hline 22 . & $\begin{array}{l}61 \text { Cyg A } \\
61 \text { Byg B }\end{array}$ & $\begin{array}{ll}21 & 04.7 \\
21 & 04.7\end{array}$ & $\begin{array}{ll}+38 & 30 \\
+38 & 30\end{array}$ & $\begin{array}{ll}21 & 06.9 \\
21 & 06.9\end{array}$ & $\begin{array}{ll}+38 & 44 \\
+38 & 44\end{array}$ & $\begin{array}{l}5.19 \\
6.02\end{array}$ & $\begin{array}{l}6.38 \\
7.40\end{array}$ & $\begin{array}{l}1.19 \\
1.38\end{array}$ \\
\hline 23 . & $\varepsilon$ Ind & 2159.5 & -5700 & 2203.4 & -5647 & 4.67 & 5.73 & 1.06 \\
\hline 24. & $\alpha$ Gru & 2205.1 & $\begin{array}{lll}-47 & 12\end{array}$ & $22 \quad 08.2$ & -4658 & 1.73 & 1.60 & -0.13 \\
\hline 25. & HD 216803 & 2253.6 & -3150 & 2256.4 & -3134 & 6.49 & 7.59 & 1.10 \\
\hline 26. & a PsA A & 2254.9 & $-29 \quad 53$ & 2257.6 & -2937 & 1.16 & 1.25 & 0.09 \\
\hline
\end{tabular}


Remarks

1. Nearest $\delta$ Scuti-type variable star.

2. Nearest evolved B star.

3. Brightest member of the nearby Per cluster.

4. Nearby dK star.

5. Nearest late gK star.

6. Nearest unevolved single dF star.

7. Distant blue supergiant; true parallax is $<0: 002$.

8. Distant red supergiant; true parallax is $<0: 002$.

9. Nearest supergiant.

10. Distant supergiant; true parallax is $<0$ ".002.

11. Nearest normal giant.

12. Nearest $B$ main sequence star.

13. Groombridge 1830 ; neaby sdG star.

14. Nearby A0V standard star; member of UMa cluster.

15. Nearby A3V standard star; member of UMa cluster.

16. Weak-line G0V star, otherwise nearly identical to No. 17 .

17. Strong-line G0V star, otherwise nearly identical to No. 16 .

18. Nearby old subgiant star.

19. Nearest high-velocity giant star.

20. Nearby A0V flux-calibration standard star.

21. Distant supergiant; true parallax is $<0.002$.

22. Nearby pair of high-velocity dK stars.

One of the pair may show orbital motion.

23. Nearby single dK star.

24. Nearby $B$ main sequence star.

25. $\alpha$ PsA B; proper motion dK companion to No. 26 .

26. Nearest normal dA star.- 
The Commission expressed its thanks to the Working Group.

The Commission also approved the report of its working group on the identification of radio/optical astrometric sources and thanked them for their work. The following is a summary of their report:

\section{SUMMARY OF REPORT OF I.A.U. COMMISSION 24 WORKING GROUP ON THE IDENTIFICATION OF RADIO/OPTICAL ASTROMETRIC SOURCES}

\section{A. N. Argue, Chairman}

The objective is to select suitable sources to act as benchmark in establishing a coordinate reference frame based on extragalactic objects. The system must be accessible to both radio and optical astrometry, therefore objects must be suitable for both methods. Approximately 100 objects uniformly spread over the sky are required.

With only a very small number of exceptions, extragalactic sources are too faint for HIPPARCOS, so that bootstrapping by ground based telescopes or, in particular, by Space Telescope will be necessary. Coordinate with Space Telescope requires

(i) Selection of 100 sources

\section{(ii) Selection of HIPPARCHOS bootstrap stars}

(i) A data-base is being compiled at the Department of Astronomy, U. of Texas at Austin, containing 600 sources with radio and optical data. The data are being continually augmented and updated, particularly in the radio domains, but the liklihood is that most of the objects finally selected already feature in this data-base.

(ii) the most likely one-third of those sources in (i) have been tenatively selected, and suitable bootstrap stars identified as HIPPARCOS candidates. These candidates must conform to certain well defined criteria as to cleanliness etc. to be acceptable to HIPPARCOS and Space Telescope. Following careful examination on Sky Survey prints they are being tested for absence of duplicity within the range 0.3 to 15 arc sec at Hamburg, and for absence of structure at the resolution limit of Space Telecope by speckle interferometry by groups at Lowell Observatory and Imperial College, London.

The Commission endorsed the proposal to hold an IAU Symposium entitled "Astrometric Techniques" from 9-12 January in Merida, Venezuela. (The organization of this Symposium was subsequently approved by the Executive Committee of the IAU.) The Commission voted to continue its joint Working Group on the Stellar Reference Frame (H. Eichhorn, chairman) with the same composition as in the triennium just passed.

The president appointed Messrs. Murray, Lutz and Stock to function as a Resolution Committee, charging it especially with solicitation for suggestions how this Commission could help avert the threatened closing of the Sydney (Australia) Observatory. During the meeting of August 19, D. King from the Sydney Observatory reported that the Observatory had, in fact, already been closed, but that the $12^{\circ}$ of the Sydney photographic catalogue which had already been measured would be reduced by April 1983, and suggested that Commission support best be directed toward urging the government of New South Wales to keep on the payroll those astronomers who were formerly on the staff of the Sydney Observatory, so that they could continue and finish the ongoing work. Such a resolution passed unanimously. The incoming president (Gliese) was asked to convey the best wishes of the Commission to the Lembang Observatory in Java on the occasion of its anniversary.

In the meeting on 19 August, scientific papers were presented by W. J. Luyten on Catalogues of Large Proper Motion, by T. Corbin on the reference stars for the Halley Watch: 
The USNO is committed to provide improved positions for the Astrometry Network of the International Halley Watch covering the path from $21^{\mathrm{h}} 00^{\mathrm{m}},-10.7$ to $18^{\mathrm{h}} 49^{\mathrm{m}},-35.0$. These are the predicted positions of the comet on 10 February 1986 and 30 March 1986, respectively. It is intended that the reference stars will cover an area extending $2^{\circ}$ on either side of the comet's path with an average density of about 5 per square degree. The stars have been chosen from the SAO and are all in the magnitude range $6.0 \mathrm{~m} 8.9$ except for a few fainter stars that were needed to fill gaps in the distribution. All known double stars that meet the conditions $\mathrm{m}_{2}-\mathrm{m}_{1}<4.0$, and $2{ }^{\prime} 0 \leqq \mathrm{~d}=\left[54^{\prime \prime}-2\left(\mathrm{~m}_{1}+\mathrm{m}_{2}\right)\right]$ have also been eliminated. Here $m_{1}$ and $m_{2}$ are the magnitudes of the two components and $d$ is their separation.

This was followed by a paper by T. Corbin and G. Douglass on the astrographic catalogue: The U.S. Naval Observatory is currently contributing to the effort to make all of the AC available in machine readable form. Strasbourg has completed the region $-2^{\circ}$ to $+32^{\circ}$ and has kindly provided us with a copy of the data. We have finished $-4^{\circ}$ to $-12^{\circ}$ and $-41^{\circ}$ to $-52^{\circ}$. Our efforts will now concentrate on the $-12^{\circ}$ through $-40^{\circ}$ region. The data will be used at USNO to provide finding positions for the measurement of the 8" Dual Astrograph plates. Finally, the AC positions will be reduced to the system of the FK4 through the use of a reference system based on the IRS (International Reference Stars) positions and proper motions.

J. Stock gave a paper on plate overlap reduction by using spherical projection; Zhao one on Astronomy at the Shanghai Observatory, and $\mathrm{P}$. Hemenway delivered a paper on HIPPARCOS.

During the Scientific Meeting of 23 August, papers were presented by A. Reiz, A. Nobili and C. Dahn, the latter on parallax measurements of faint stars using CCD cameras.

This is an abstract of Nobili's paper: Geophysical use of synchronous Earth satellites astrometry. Geosynchronous satellites currently used for telecommunication purposes can be easily tracked with moderate size large field telescopes measuring the satellites angular coordinates with respect to some catalogue field stars and the accuracy is between 1 and 2 aresec. These data can be analyzed to measure the ellipticity of the Earth's equator. A recent paper (Catalano, McCrosky, Milani and Nobili, 1982, Journal of Geophysical Research, in press) shows that the organization of an international optical tracking campaign with the accuracy mentioned above would allow an improvement in the knowledge of the long wavelength geoid by at least one order of magnitude, that is very interesting for many geophysical purposes.

The following paper was transmitted with a request for publication:

\title{
A DETERMINATION OF STELLAR PROPER MOTIONS WITH RESPECT TO GALAXIES AT PULKOVO
}

\author{
N. M. Bronnikova, A. N. Deutsch, N. V. Fatchikhin \\ O. N. Orlova and N. A. Shakht \\ Pulkovo Observatory, U.S.S.R.
}

Absolute proper motions of stars determined with respect to galaxies can be used both for determination of the solar motion parameters, galactic rotation constants and for a calculation of the correction to the precession constant.

At present four programs for the measurement of stellar proper motion with respect to galaxies are in progress. The LICK [1] and Yale-Columbia [2] programs use 51-cm aperture astrographs, Lohrmann program [3] uses the 2-m Reflecting Telescope. The program of the Pulkovo observatory [4] uses various Normal Astrographs and other more long-focus instruments at a few observatories in the northern and southern hemispheres. 
The Pulkovo zone envolves 157 areas within $-5^{\circ}$ to $+90^{\circ}$ in declination [5]. Besides areas with galaxies the Pulkovo program foresees separate photographs of the fundamental stars of the catalogue of faint stars (F KSZ. The list of $240 \mathrm{FKSZ}$ was published by A. N. Deutsch [6].

A number of papers have been published dealing with the proper motions of stars related to galaxies, obtained at Pulkovo by N. V. Fatschichin [7], at Taschkent by A. G. Rachimiv [8], at Golosseevo by S. P. Rybka [9] and N. V. Harchenko [10].

At present a new catalogue of absolute proper motion of stars from observation with the Pulkovo Normal Astrograph is nearing completion. We have got $95 \%$ of second-epoch plates with galaxies and $73 \%$ of second-epoch plates with FKSZ. The epoch differences are more than 20 years. We hope to obtain all second-epoch plates in 1983 and then we shall have 3 plate pairs for each of 157 areas with galaxies and 2 pairs for 24 areas with FKSZ. All plates with FKSZ and one of the three pairs with galaxies is taken with diffraction gratting.

270 plate pairs for 135 areas with galaxies have been measured. For 80000 stars up to the 16 magnitudes have been determined relative proper motion. The reductions have been calculated for a conversion of relative proper motions to absolute ones using galaxies, stars of the AGK3 catalogue on 135 areas. Besides we applied a statistical method using Binnendyk's secular parallaxes.

Using the reduction on 20 areas distributed on the hemisphere uniformly we have calculated the secular parallaxes for stars $13^{\mathrm{m}} \cdot 7$ and 15.7 . The meaning parallaxes are given next table.

\begin{tabular}{|c|c|c|}
\hline $\mathrm{m} / \mathrm{b}$ & $27^{\circ}$ & $61^{\circ}$ \\
\hline $13 ! 18$ & $+0 ! 0089$ & \\
\hline & $\begin{array}{r} \pm \\
+0 ! 0059\end{array}$ & \\
\hline & \pm & \\
\hline
\end{tabular}

where $b$ is a galactic latitude, $m$ is a magnitude of stars.

The average number of well measured galaxies in an area is 4, the stars AGK3 is 20 . According to preliminary data [11.12] the probable error of a star of $14^{\mathrm{m}}-15^{\mathrm{m}}$ as determined from one pair equals +0!006. The error of the measurement of galaxies is about twice as large as that of stars of $14^{\mathrm{m}}-15^{\mathrm{m}}$. The probable error of the reduction determined using 3 pairs is $\pm 0.003[13]$.

\section{References}

Deutsch, A.N. 1954. Trans. IAU, 8, p. 789.

Deutsch, A.N. 1980. Isvestia Glavn. Astr. Obs. 198, p. 124.

Deutsch, A.N., Lavdovsky, V.V., Fatschichin N.V. 1955. Isvestia Glavn. Astr. Obs., 154, p. 14.

Deutsch, A.N., Lavdovsky, V.V. and Orlova, O.N. 1975. In book "Sovremennye problemy Astrometrii". p. 214, Moskwa.

Deutsch, A.N., Lavdovsky, V.V. and Orlova, O.N. 1978. In book "Novye idei v Astrometrii". p. 72 , Leningrad.

Fatschikhin, N.V. 1974. Trudy Glavn. Astr. Obs., 81, p. 2.

Fatchikhin, N.V. 1982. Izvestia Glavn. Astr. Obs., 200, 107. Pulkovo, 1982.

Harchenko, N.V. 1980. In book "Astrometria i Astrophizika". 35, p. 3, Kiev.

Harchenko, N.V. 1980. In book "Astrometria i Astrophizika". 42, p. 70, Kiev.

Rachimov, A.G. 1978. In book "Kinematisheski i dinamisheskie harakteristiki otdelnych zvezdnych system". 109,59 , Tashkent.

Rybka, S.P. 1978. In book "Astrometria i Astrophizika", 35, p. 3, Kiev.

Sanding, H.-U. 1976. Mitteil. Lohrman Obs., N33, p. 943.

Wesselink, A.J. 1974. "New Problem in Astrometry". Ed. W. Gliese, p. 201.

Wright, W.H. 1950. Proc. Amer. Phil. Soc., 94, p. 1. 
COMMISSION 25: STELLAR PHOTOMETRY AND POLARIMETRY (PHOTOMETRIE ET POLARIMETRIE STELLAIRES)

Reports of Meetings 18 and 21 August 1982

PRESIDENT : J.A. Graham

VICE-PRESIDENT: J. Tinbergen
SECRETARIES: J.A. Graham

M.F. McCarthy

At the Patras General Assembly, activities of Commission 25 consisted of 5 sessions. 3 sessions were joint meetings with other Commissions and 2 were held by Commission 25 alone. Reports of two of the joint meetings, "Systematic Effects in Abundance Determinations for Meta1-Poor Stars" and "Stellar Absolute Energy Distributions" will be given elsewhere. Here, we report on the joint meeting with Commission 37, "Photometry in Crowded Fields" and the two Commission 25 meetings "New Standard Stars Results" and "Commission 25 Business".

\section{PHOTOMETRY IN CROWDED FIELDS}

This joint meeting was with Commission 37 (Stars Clusters and Associations). It was organized by $\mathrm{Dr}$. Alan Penny (UK) who also presided except during the period of his own presentation when the undersigned took the chair. The following talks were given.

1) Photometry of Magellanic Cloud Clusters -- M.F. Walker (USA)

Dr. Walker outlined the program undertaken in collaboration with A. Blecha and J. Anderson to study Magellanic Cloud clusters with the Danish $1.5 \mathrm{~m}$ telescope on La Silla. A McMulien electronographic camera is used to obtain electrographs which are subsequently analyzed with either a Joyce Lobel measuring machine or the Geneva two-dimensional scanning system. Results for the cluster Hodge 11 were presented and a discussion given of methods to deal with the problem of field crowding.

\section{2) The KPNO Rich Field Program -- J.R. Mould (USA)}

The Kitt Peak Rich Field program has become widely used in deriving magnitudes and colours from digital images. Dr. Mould gave an account of its operation from the user's viewpoint. All images in the field are replicas of some point-spread function which is determined as a first step towards subsequent analysis. The program accounts for elongation of images if present.

3) RR Lyrae Stars in the Central Bulge of Our Galaxy '-- B. Helt (Denmark)

Dr. Helt described a search for RR Lyrae variable stars in the central regions of our Galaxy. The Danish $1.5 \mathrm{~m}$ telescope and $40 \mathrm{~mm}$ electronic camera was used to observe 3 fields, each $10^{\prime}$ diameter. The search was carried out both by blinking plates and by forming positive/negative composite images. Many new variables have been discovered in the Sgr I field. Dr. Helt emphasized that it is essential to always check the linearity of the electronic camera by observing, for example, standard sequences during each observing run.

4) Gaussians and Lorentzians to Fit a Multiple Stellar Image -- A. Penny (UK)

This talk described the use of single 2D Gaussian profiles and multiple 2D Franz-Lorentz profiles by a suite of programs on the Royal Greenwich Observatory Starlink node. The single Gaussian was surprisingly resistant to the presence of a nearby star. The multiple Lorentz profiles fitted actual star images well. 


\section{5) Crowded Field Electronography -- P. Linde (Sweden)}

In this paper, the techniques used at Lund Observatory for dealing with photometry in crowded fields were discussed. Methods for fitting and checking the point-spread function were described and reference was made to the linearity and the relation between photoelectric and electronographic magnitudes.

J.A. Graham

18 August 1982

\section{NEW STANDARD STAR RESULTS}

The scientific session of Commission 25 on August 18 was concerned with "New Standard Star Results". With the many new area detectors available, a strong need has arisen for faint photometric and polarimetric standard stars which can serve as calibration standards. Preferably such stars should be distributed over a wide range of magnitude, be spread over the sky sufficiently so that some are accessible at any time, and be well tied into established photometric systems.

A new Catalogue of Photometric Sequences is being prepared by $N$. Argue (UK), E. Miller (USA) and W. Warren Jr. (USA). N. Argue was prevented by unforseen circumstances from presenting a report on this project but later communicated results about progress. The work is actually the third supplement to two earlier catalogues. It is complete now to 1982.0 and will contain 1271 new sequences, mostly UBV(RI) to limiting magnitude 23. The magnetic tape will soon be available from Wayne Warren at the World Data Center, NASA-Goddard. Later on, copies of the tape will be available from the Centre de Données Stellaires, Strasbourg and a printed version will be circulated.

Recent work concerning UBVRI Photoelectric Sequences Around the Celestial Equator was described by A. Lando1t (USA). Observations have been made of 223 stars on the UBVRI systems of Johnson and Cousins. The stars average 20.7 measures each on 12.2 different nights and fall in the magnitude range $7<\mathrm{V}<12.5$.

Because of illness, D. Kilkenny (S. Africa) was unable to present his paper "Standard Star Work at the South African Astronomical Observatory". In his place, I. Glass (S. Africa) gave a brief summary of programs in progress at SAAO and presented some observations of standard stars in the infrared JHKL bands.

Finally, M. Breger (USA) gave an account of work done on Highly Precise Standards for Polarization Measurements. The widely used polarized and unpolarized standards by Serkowski have been extensively reobserved to extend their accuracy to a few parts in $10^{4}$ in $p(\lambda)$. Among polarized standards, 3 early-type supergiants (55 Cyg, $9 \mathrm{Gem}$, HD 183143) show definite polarimetric variability. The unpolarized standard HD 202573 is slightly polarized with $\mathrm{p}(\mathrm{V})=0.054 \pm 0.010 \%$. These stars should not be used as standards. Special attention was given to the rotation of position angle with wavelength in this work.

J.A. Graham

18 August, 1982

\section{COMMISSION 25 BUSINESS MEETING}

The President (J.A. Graham) called the meeting to order at 9.05 a.m. and appointed the undersigned to serve as Recording Secretary for the session. About 25 members were present. President Graham announced the following Agenda which was approved unanimously. 
1) Opening Remarks. Commemoration of Departed Colleagues.

2) Report of Commission in Trans. IAU XVIIIA.

3) New Members of the Commission.

4) New Commission Officials.

5) Resolutions.

6) Scientific Results.

The President commemorated our departed President of the Union, Dr. M.K. Vainu Bappu and recalled his early work in $\mathrm{H} \gamma$ photometry. Though he was a never a member of Commission 25, Dr. Bappu had an initial and continuing interest in our field and we will miss his presence and direction. The President also commemorated the departure from us of Dr. Harold L. Johnson, whose contributions to photometry were so many and so important for our science, Dr. Graham characterized Johnson's spirit as one which, with excellence as his norm, was alert and active. He was always a person who looked for and found new fields to conquer. Next President Graham eulogized our departed colleague $\mathrm{K}$. Serkowski whom he called "one of our polarimetric pioneers". Then President Graham invited all members to stand for a moment of silence to honor the memory of these departed colleagues.

The President's Triennial Report was next considered. Pres. Graham thanked all members who contributed and acknowledged especially the assistance of Drs. Bessel1, Chugajnov, Hauck, Hyland and Tinbergen in its preparation. M. McCarthy moved that the report be accepted; this was seconded by $T$. Gehrels and approved.

President Graham next read a list of new officers proposed to serve the Commission during the next three years; this read as follows:

President : J. Tinbergen

Vice-President: F. Rufener

Scientific Organizing Committee: M. Bessel1, P. Chugajnov, J. Dachs, P. Edwards, J. Graham, B. Hauck, D. Kilkenny, A. Landolt, I. McLean, V. Piirola, N. Shakhovskoj.

M. Besse1l pointed out that there was no person on the SOC whose work was chiefly infrared photometry. I. Glass (S. Africa) was suggested and added to the above list. Approval of the nomination list was moved formally by A. Landolt, seconded by M. McCarthy and carried. The President then thanked the retiring members of the SOC for their years of service.

The President suggested that recommendations from members could be placed in this account of the proceedings rather than be submitted to the formalities of the Resolutions Committee, unless there was some pressing item of importance. There were no resolutions to be submitted formally on this occasion.

Vice-President Tinbergen, President-Elect of Commission 25 asked members to send him notes for the periodic progress reports which he plans to circulate to members of the Commission. The new address of our incoming President is as follows:

\author{
Dr. J. Tinbergen \\ Kapteyn Sterrenwacht Werkgroep \\ Mensingheweg 20 \\ $9301 \mathrm{KA}$ - Roden \\ THE NETHERLANDS
}

The final business matter was the reading of the list of new members. These are L. Baldinelli, B. Carney, D. Hall, M. Das, J. Denoyelle, J.C. Forte, I.S. Glass, A. Grauer, M. Grenon, H. Hensberge, R. Hilditch, D. Kilkenny, Z. Kviz, P. Laskarides, R. Lenzen, V. Oskanyan, A. Penny, R. Pfeiffer, A.G.D. Philip, D. Sullivan,

H. Trodahl. Consultants for the coming triennium are J-Y. Daniel and E. Miller. 
A rousing round of applause was offered to thank John Graham for his splendid service of Commission 25. Congratulations and promises of support were also offered to our new President Jaap Tinbergen as he begins his work for us all.

The final portion ( 40 mins) of the session was devoted to scientific presentations. The undersigned wishes to point out that our meetings were attended by many guest members of the IAU and Invited Participants thus demonstrating the appeal and importance of the twofold branches of our science: stellar photometry and polarimetry.

Dr. E.F. Milone (Canada) described the new photometer in use with the 16" telescope at Calgary. The photometer incorporates a rapid alternate detecting system. It uses a chopping secondary mirror whose position angle can be varied 360 degrees in the plane of the sky. Four-channel pulse counting electronics are synchronized to four positions of the secondary mirror permitting effectively simultaneous measurement of a comparison star, variable star and two sky backgrounds using a single photomultiplier, filter and aperture. The system adequately compensates for the effects of transparency, sky brightness and detector sensitivity variations and has been successfully used with a cloud cover as much as 3 magnitudes.

Next, Dr. P. Edwards (New Zealand) spoke on Atmospheric Extinction Measurements. He described cooperative work undertaken in collaboration with the meteorologists to determine extinction values in UBV, uvby and narrow band regions. Dr. Edwards outlined the procedures followed and also mentioned the complications caused this year by the eruption of 2 volcanoes (one in Mexico and one in Java). Very large extinction values were noted in Texas and Hawaii by some commission members. It was recommended that, before blithely applying mean extinction coefficients, all colleagues should be careful to test the quality of the night with adequate and necessary measurement of standard stars. It was noted that a working group had been formed within Commission 50 (Protection of Existing and Potential observing Sites) to determine extinction effects due to volcanoes. There was an active discussion of the importance of archival storage and possible publication of extinction data.

The scientific portion of the session concluded with a lively talk by Dr. Tom Gehrels (USA) about the program of the Spacewatch Camera. This presently is the $0.9 \mathrm{~m}$ Newtonian telescope of the Steward Observatory on Kitt Peak. The plan is to replace it with a specially designed $1.8 \mathrm{~m}$ prime focus reflector. It will use a CCD detector in the continuous scanning mode primarily for the discovery of near earth asteroids but also for stellar and galactic observations. During the light part of the moon, the telescope is to be used with a radial velocity spectrometer for the search of planets of other stars. This program was initiated by Krzysztof Serkowski and after his death continued by Robert McMillan.

This concludes the Secretary's Report of the Commission 25 Business Meeting.

Martin F. McCarthy, S.J. 21 August 1982 
Report of Meetings, 18 and 23 August 1982

PRESIDENT: 0. G. Franz

SECRETARY : J. Dommanget

\section{August 1982}

The President opened the meeting and welcomed those in attendance. J. Dommanget was appointed to serve as Secretary. All present stood briefly in silence to honor the memory of two distinguished colleagues, P. M. Djurkovic and U. Güntzel-Lingner, lost by death since the 1979 meeting.

1. Commission of ficers and membership

By written nomination and secret balloting, the Comission endorsed the following officers for 1982-1985:

President: M. G. Fracastoro

Vice President: K. D. Rakos

Organizing Committee: J. Dormanget, W. D. Heintz, and H. A. McAl ister

Continuing for a second term on the Organizing Comittee will be A. Poveda and C. D. Scarfe. Franz will serve one term as an ex-officio member.

The President announced the resignation from the Commission of A. S. da Silva and M. C. Johnson. He then presented, and the Commission endorsed, the following list of proposed new members: E. Cabrita, R. B. Culver, A. N. Dadaev, F. C. Fekel, 0. E. Ferrer, C. A. Hernandez, G. Ishida, M. I. Kumsiashvili, N. L. Magalashvili, R. Pannunzio, G. N. Salukvadze, A. M. Shulberg, M. Tapia, A. Valbousquet, and K. A. van der Hucht.

2. Double star work with the 26-inch Johannesburg refractor

At the invitation of the President, J. Hers attended the meeting and commented briefly on the status of the Johannesburg refractor. He expressed his willingness to help explore conditions under which some use of this intrument for visual double star work might be resumed. Among these conditions would have to be expressions of real interest by double star observers from abroad to come to Johannesburg for short or longer observing visits if given some financial help. Statements of interest and specific information on necessary assistance (e.g., travel expenses, living expenses, accommodations, etc.) should be communicated directly to $\mathrm{J}$. Hers, Post Office Box 48, Sedgefield, South Africa 6573.

\section{Name of Commission 26}

A proposal by Dormanget to change the name of the Commission from "Double Stars (Etoiles Doubles)" to "Double and Multiple Stars (Etojles Doubles et Multiples)" was adopted unanimously and forwarded to the Secretary General for formal action by the General Assembly during its second session at Patras.

\section{Proceedings of IAU Colloguium No. 62}

R. Harrington reported that some authors of contributed papers have shortened 
their manuscripts to reduce printing costs, while others have agreed to pay charges for excess pages. Publication of the proceedings still depends on the availability of the necessary funding.

\section{Nomenclature of components of multiple systems}

At the request of the President, Heintz submitted a statement concerning the designation of the components of multiple stars. Following several comments by Commission members, the President recommended that further discussion be postponed until the second session of the Commission to allow all interested members time to review the problem and to analyze the statement of Heintz.

\section{Supplementary Rules (By-1aws)}

The President recalled that during its meeting of 19 May 1981 at Flagstaff, Arizona, the Organizing Comittee discussed and endorsed new Supplementary Rules (By-laws) to define and clarify the nominating and balloting procedures for Commission offices. These proposed by-laws, intended not to replace or alter, but to supplement the existing Internal Working Rules, were subsequently submitted to all Commission members for their review and written comments. While those who responded expressed unanimous support for the intent of these by-laws, some difficulties and certain inconsistencies with existing working rules were noted, indicating the need for further deliberations before a final version of the by-laws could be prepared.

The President's remarks were followed by extensive and often spirited discussion, particularly concerning voting by proxy and the problems which balloting by mail may pose if unforeseen and unpredictable situations should arise. The Commission then decided, by unanimous vote of those present and voting, to adopt the new by-laws "in principle." The retiring president was directed to form an ad hoc committee for the purpose of revising the proposed Supplementary Rules, perhaps combining them with existing Internal Working Rules, and submitting a revised text to the Commission for ratification as soon as possible after the Patras General Assembly.

\section{August 1982}

\section{SCIENTIFIC PRESENTATIONS}

1. A. H. Batten: Recent Results on $\Sigma 2367$ and 70 Ophiuchi

2. C. E. Worley: Status Report on the Double Star Index, Observation, and Orbit Catalogs

3. J. Dommanget: A New Catalog of Ephemerides of Radial Velocities for Orbital Visual Binaries

: The Observation of Radial Velocities of Visual Binaries

4. C. D. Scarfe: Zeta Hercul is - The Third Dimension

\section{ADMINISTRATIVE MATTERS}

\section{Co-sponsorship of symposia and colloquia} meetings:

The Commission voted to offer co-sponsorship for the following proposed

a) Symposium on "Calibration in Astronomy"; tentative plans being coordinated by A. H. Batten. 
b) Colloquium on "MK Spectral Classification: Criteria and Applications"; R. F. Garrison, Chairman, Colloquium Organizing Committee.

c) Colloquium on selected topics concerning the "Masses and Dynamics of Double and Multiple Stars," to be held at Lembang in June 1983; B. Hidayat, Chairman, Local Organizing Committee.

\section{Next IAU Double Star Colloquium}

A tentative proposal to hold a "Double Star Colloquium" in 1984 in Italy was presented by Fracastoro and accepted by the Commission. Precise date, location, and spectfic topic will be determined by a colloquium organizing committee at a later date.

\section{Nomenclature of components of multiple stars}

In response to the statement presented by Heintz during the session of 18 August 1982, separate proposals on the designation of components of multiple systems were presented by Couteau and Valbousquet. Heintz reiterated that the purpose of his statement was not to make a specific proposal, but to call attention to this problem and the need for its consideration and solution. Since it was clear that such a solution could not be found during the remainder of the Commission meeting, the President urged those most interested in and affected by this problem to confer directiy and to develop a mutually acceptable nomenclature. 
COMMISSION 27: VARIABLE STARS (ETOILES VARIABLES)

Report of Meetings: August 19 and 23, 1982

PRESIDENT: J.D. Fernie

SECRETARY: Nancy R. Evans

BUSINESS AND SCIENTIFIC MEETING, August 191982

1. Nominations. 1982-85:

The commission agreed to nominate the following Organizing Committee for

President: N. Baker

Vice-President: B. Szeldl

Members: M. Breger, A.N. Cox, R.E. Gershberg, M. Jerzykiewicz,

L.N. Mavridis, L.N. Mirzoyan, J.R. Percy, A.M. van Genderen,

B. Warner, J.D. Fernie (ex-officio).

2. New Members.

It was agreed that the executive be authorized to review the 1 ist of proposed new commisston members and decide on their admission.

3. General Catalogue of Variable Stars.

The President read a report by Dr. Samus' concerning the continuing work on the GCVS. The fourth edition of the catalogue is well underway, and will include a listing of extragalactic variables and one of cross-listings. The new Catalogue of Suspected Variables, containing 14811 stars, has been published, and a copy was shown at the meeting. The Catalogue is also available: on magnetic tape through the Strasbourg Centre, as will be the $4 \mathrm{th}$ edition of the GCVS. The total number of designated variable stars now amounts to 28254 .

The Commission is much indebted to the Sternberg Astronomical Institute for its work on these catalogues.

4. Informatioil Bulletin on Variables Stars.

A report by the editor of IBVS, Dr. Szeidl, was read to the meeting. Dr. Szeidl noted that as of August 6, 1982, 2184 issues of the Bulletin had been published, including more than 500 since the previous General Assembly. With only Dr. Szeidl, a secretary, and Dr. Szabados available to produce the Builetin, the task is a heavy one, and Dr. Szeidl emphasized that material submitted to the Builetin must be in camera-ready form, particulariy if the turn-around time of two to four weeks is to be maintained.

Dr. Szeidl proposed that the Bulletin continue to publish material on eclipsing binarles, a proposal wholeheartedly supported by the meeting. It was moved and unanimously agreed that Dr. Szeidl be thanked for his production of the Bulletin and asked to continue the work.

\section{Archives of Unpub1ished Data.}

Dr. Breger reminded the meeting of the existence of the archives for 
unpublished photoelectric data, and the manner in which the system works. Summaries of existing data files are published in the PASP every eighteen months. A third data bank, that at the Strasbourg Centre, has been added to the system.

6. Hipparchos Satellite Project.

Dr. C. Jaschek pointed out to the meeting that the Tycho portion of the Hipparchos satellite is expected to obtain photoelectric magnitudes for many thousands of the brightest stars over a three-year period, with typically fifty observations per star. This could be of immense benefit to varlable star observers. Technical details are available from Dr. E. Hoeg of the Copenhagen Observatory.

7. Cepheid Colloquium.

The President announced that it was hoped to celebrate the 200th anniversary of the discovery of cepheid variables by holding an IAU Colloquium on cepheids at the University of Toronto/David Dunlap Observatory probab1y in August 1984.

\section{Scientific Session.}

The meeting closed with three short review papers:

A. Maeder: Hubble-Sandage Variables

J. Percy : Early-type Variables

A. Cox : Double-mode RR Lyrae Stars

MEETING OF FLARE-STAR WORKING GROUP, August 23, 1982

The meeting was arranged by $\mathrm{Dr}$. Mavridis and was held at the Stephanion observatory. Sixteen persons attended. The following papers were presented:
V.A. Ambartsumian: The Main Aims of the Program of Observations of Flare
L.N. Mirzoyan : Some Results of Three-Colour Photographic Observations of Flare Stars in Open Clusters.
P.V. Kulkarni : Infrared Flare Activity in the Rapid Burster 1730-333.
C.A.O. Torres : Evolutionary Status of the Pleiades from the K-type BY Dra Stars.
B.R.P. Pettersen : Time Scales of Variations in Continuum and Hydrogen Emission Lines During Flares.
L.N. Mavridis, G. Asteriadis, and F. Mahmoud: Long-Term Variations of the Flare Activity and Quiet-state Luminosity of the Flare Stars EV Lac and BY Dra.

SCIENTIFIC MEETING, August $23, ' 1982$

An informal scientific session produced the following speakers and papers:

D. Kilkenny: The RCB Stars.

P.W. Hill : Extreme Helium Stars.

N.R. Evans : Recent Observations of SU Cas.

W. Gieren : Continued Discussion of SU Cas.

C.T. Bolton: V986 Oph.

J.A. Mattel: The AAVSO Data Bank and Publications.

J. Rountree: The BW Vu1 Observational Campaign.

S. Barcza : Problems in Calibrating RR Lyrae UBV Colours in Te and log $g$.

G. Bakos : Light Variations in 57 Peg.

M. Frolov : The Classification of $\delta$ Scuti Stars.

J. Cuypers : The $\beta$ Cephei Star $\beta$ Cru. 
COMMISSION 28: GALAXIES (GALAXIES)

Report of Meetings, 18, 19, 20, 23, 24, and 25 August 1982

PRESIDENT: B. E. Westerlund SECRETARY: N. Bergvall

Commission 28 was involved in twelve sessions, three of them all-day joint discussions:

Evolution in old Stellar Populations in Galaxies (Commissions 25, 28, 29, $33,34,35,37,45,47)$, August 19.

The Extragalactic Distance Scale and the Hubble Constant (25, 28, 40, 45, 47, 48), August 23.

Active Galactic Nuclei $(28,40,48)$, August 18.

Details of these joint discussions will appear in Highlights of Astronomy, volume 6 . Of the remaining sessions, three were joint meetings: Dark Haloes

of Galaxies (with Commission 33), Beams and Jets in Extragalactic Sources (with Commission 40), and The Local Supercluster and its Environment (with Commission 47). The remaining sessions were Commission 28 alone. Programs for these meetings and abstracts of some of the papers presented follow the minutes of the business meeting below. Other abstracts or summaries may be found in the Highlights of Astronomy.

\section{August 1982}

\section{BUSINESS MEETING}

\section{NEW OFFICERS AND MEMBERS}

The Commission unamimously approved the election of the new President, V. C. Rubin, and Vice-President, P. C. van der Kruit. The President proposed, and the Commission unanimously accepted, the list of names proposed for the new Organizing Committee.

The list of IAU members who wish to join Commission 28 was read and adopted. A second list of proposed new members to the Union, wishing to join Commission 28, was read and adopted, after a short discussion.

II. REPORT OF THE COMMISSION

The President asked for any comments regarding the report of the Commission, which had been distributed to the Commission members present in Patras before the meeting. The report was adopted without discussion.

\section{THE WORKING GROUPS}

A large amount of administration work goes into the Working Groups and compared to this some members considered the scientific benefit to be too meager. As arguments against this, the importance of the Working Groups for initializing cooperation between different fields and for circulating abstracts was stressed. After some discussion the Commission, recognizing the importance of circulating information, recommended that the Working Groups should continue to exist.

\section{RESOLUTIONS AND RECOMMENDATIONS}

D. Lynden-Bell pointed out the fact that, although precession influences a measured orientation of a galaxy, very few observers quote the epoch they 
have used, when presenting their results. The problem was discussed, and it was recommended that the epoch should be quoted whenever a position angle is published.

$V$. Rubin drew attention to the ambiguity existing in connection with the definition of the inclination angle of a galaxy. After a short discussion the following internal resolution was adopted:

"Define the inclination $i$ of a galaxy such that $i=0$ when the angle between the line-of-sight to the galaxy and the rotation axis of the galaxy equals $0^{\circ}$ ( $i$.e. face on), and $i=90^{\circ}$ when the angle between the line-of-sight and the rotation axis of the galaxy equals $90^{\circ}$ (i.e. edge-on)".

\section{MISCELIANEOUS}

The problem of defining coordinates for objects within a galaxy was discussed. A group, consisting of H. R. Dickel, P. W. Hodge, D. Lynden-Bell and S. M. Simkin was formed in order to treat the problem in more detail.

The question of whether supernovae should be included in Commission 28 was discussed. It was suggested that a Working Group on superncvae should be established. The Organizing Committee was asked to find a proper solution to the problem.

T. Kinman had asked the Commission to urge all observers to publish their redshifts as soon as they are available. It was recognized that systematic collection of redshift data is in progress at a few places. The Commission recommended the incoming President to contact H. Rood in order to encourage him to continue his collection of redshifts and to publish them at regular intervals.

$K$. Freeman reported from the Bescancon symposium "Internal Kinematics and Dynamics of Galaxies".

\section{PROGRAMS OF SCIENTIFIC SESSIONS}

NEW RESULTS IN EXTRAGALACTIC RESEARCH August 20

S. Gottesman: Neutral Hydrogen in the Dwarf Elliptical Galaxies NGC 185 and NGC 205

F. Schweizer: Merging Galaxies and the Formation of Ellipticals

H. van Woerden: Distribution and Motion of HI in Lenticular Galaxies

$H$. van Woerden and R. Warmels: Comparison of HI Diameters in Virgo Cluster and Field Galaxies

L. Dressel: NGC 315: High-Velocity Clouds in an Active Elliptical Galaxy?

$\mathrm{J}$. Hesser: Mass and Distance of NGC 5128

K. C. Freeman: Dynamics of Galactic Bulges

B. F. Burke: Gravitational Lenses and Dark Matter in the Universe

J. Hutchings: QSO- Galaxy Interactions and QSO Morphology

D. M. Worrali: The Broad-Band X-Ray Spectrum of a QSO Sample and Multifrequency Observations of BL Lac Objects

H. R. Miller: A Recent Photometric Study of Seyfert Galaxies

G. A. Gurzadyan and V. G. Gurzadyan: On the Dynamical Structure of QSOs Derived from Emission Line Data

A. Pedlar: A Ha Filament Associated with Radio Emission in NGC 1275

E. Valtaoja: A 15.7 Min Periodicity in OJ 287?

M. G. Pastoriza: Metallic Abundances of $\mathrm{E}$ and $\mathrm{SO}$ Galaxies and Globular Clusters of the LMC and SMC

Ch. J. Peterson: The Motion of the Local Group

DARK HALOES OF GALAXIES August 20 Joint Meeting with Commission 33

See Commission 33 for presented papers 
THE LOCAL SUPERCLUSTER AND ITS ENVIRONMENT August 24 Joint Meeting with

Commission 47

B. Tully: Morphology of the Local Supercluster

J. Huchra: The Dynamics of the Local Supercluster

E. Salpeter: Theoretical Models for Virgocentric Infall

G. Abell: Properties of Superclusters

J. Einasto, H. G. Corwin, J. Huchra, R. H. Miller, and M. Tarenghi: Local Cells of the Universe; Voids of Diameter $200 \mathrm{Mpc}$ (presented by M. Tarenghi)

J. Zeldovich: Remarks on the Structure of the Universe

GIANT HII COMPLEXES OUTSIDE OUR GALAXY August 24

1. Observational results and their primary interpretations in the following domains:

Radio: D. S. Heeschen

$X$-rays: L. van Speybroeck

Visible: M. Rosa

UV: P. Benvenuti

IR: C. M. Telesco

2. Confrontation with related investigations in our Galaxy and general interpretations: F. P. Israel

BEAMS AND JETS IN EXTRAGALACTIC SOURCES August 25 Joint Meeting with Commission 40

See Commission 40 for presented papers

ABSTRACTS OF SCIENTIFIC PAPERS:

S. T. Gottesman: Observations have been made in collaboration with $D$. W. Johnson of the atomic hydrogen content of the dwarf, peculiar elliptical galaxies NGC 185 and NGC 205. An angular resolution of about 1 ! 1 was obtained ith the VLA with a velocity resolution of approximately $6 \mathrm{~km} / \mathrm{sec}$.

The gas in NGC 185 does not appear to be rotating. Rather, a single not highly resolved feature was observed with a mass of $1.8 \times 10^{5} \mathrm{M}$ :

In the case of NGC 205 an extended feature with structure ${ }^{\circ}$ is found with a mass of $4.1 \times 10^{5} \mathrm{M}$. The gas appears to be in the form of a rotating disk with a maximum observêd velocity of $29 \mathrm{~km} / \mathrm{sec}$ at a radial distance of $\sim 1 ! 5$.

In each system, the centroid of the gas is significantly offset from the center of the galaxy. Also, the gas does not coincide with the well known dust clouds observed in NGC 185. In the case of $\mathrm{NGC} 205$ the gas appears to be more closely associated with the dark matter. It is difficult to understand how . Ol\% of the total mass of these galaxies has failed to coalesce at the bottom of the galaxien potential well. Perhaps the explanation is associated with the bursts of star formation that are occurring in these peculiar elliptical systems.

L. L. Dresse1: We report the detection of HI in absorption against the nuclear continuum source in the giant elliptical galaxy NGC 315. This is the first reported observation of individually resolved high-velocity clouds in or near an elliptical galaxy. The absorption line is highly blue-shifted in the reference frame of the galaxy, with a projected velocity toward the galaxy of $490 \mathrm{~km} \mathrm{~s}$. It is very strong $(244+4 \mathrm{mJy})$ and consists of two components with very narrow velocity widths of $2.5 \mathrm{~km} \mathrm{~s} \mathrm{~s}^{-1}$ (FWHM) each. The peak optical depths are 0.9 and 0.2. If the linewidths are due solely to thermal broadening, then the kinetic temperaturemust be $150 \mathrm{~K}$. The neutral gas column densities implied are $2-5 \times 10^{20}$ hydrogen atoms $\mathrm{cm}^{-2}$. These absorbing clouds are thus very similar to the aiffuse HI clouds observed in absorption in the Galaxy. We did not detect OH in absorption in these clouds to a limiting peak optical depth of 0.04 , but this result is consistent with either a galactic or extragalactic origin of the clouds. They may have condensed out of a large unobserved galactic halo of ges, or they may reside in or have been captured from the intracluster medium. 
The investigation has been carried out in collaboration with T. M. Bania and M. M. Davis.

J. E. Hesser: Spectroscopic observations with the CTIO 4-m telescope and SIT vidicon have confirmed 17 of 18 visually selected candidates as globular clusters. The cluster system, as presently known, extends to a projected radius of $\sim 36(\mathrm{D} / 5 \mathrm{Mpc}) \mathrm{kpc}$, where $\mathrm{D}$ is the distance to $\mathrm{NGC5128}$ in Mpc. Evidence has been found for a radial $B-V$ color gradient, with the redder, presumably more metal enriched, clusters lying nearer the nucleus. The heliocentric radial velocities range between $\sim 300-860 \mathrm{~km} \mathrm{~s} \mathrm{~s}^{-1}$. Star counts on UK Schmidt plates suggest a total cluster population of $\sim 600$. Observations in May, 1982, of 13 objects within the color range of the confirmed clusters but chosen without regard to possible spatial extent reveal two more clusters. This supports the prediction of the star counts concerning the presence of many more clusters than have been visually identfied by their slightly non-stellar appearance. From the velocities we estimate a mass of $\sim 10^{12} \mathrm{M}$. Scaling the combined Galactic and M31 globular cluster luminosity function $t 8^{\circ} \mathrm{N} \sim 600, E(B-V)=0.10$ mag allows a comparison with the NGC 5128 cumulative luminosity function for confirmed clusters; this comparison yields a distance of $\sim 3 \mathrm{Mpc}$.

The investigation has been carried out in collaboration with H.C. Harris, S. v.d. Bergh, and G. L. H. Harris.

Diana M. Worrall: Einstein Observatory IPC X-ray measurements of QSOs have been used by several authors to estimate the contribution of QSOs to the diffuse $X$-ray background (XRB). However, the shortage of information on quasar spectra is a major difficulty since one compares IPC fluxes essentially measured at I keV with an XRB known above $3 \mathrm{keV}$. X-ray spectra above $2 \mathrm{keV}$ are known with good statistics for the two brightest QSOs, and with less precision for a sample of 14 others. A soft spectral excess, such as is observed in BL Lac-type objects, would result in an overestimate of the QSO contribution to the XRB. Frank Marshall (Goddard Space Flight Center) and I have compared the average $\sim 7 \mathrm{keV}$ flux for an optically-selected sample of 25 QSOs detected, just above threshold, with the HEAO-I A2 experiment, with their average $\sim$ I keV flux measured with the IPC. Our preliminary analysis gives a $90 \%$ confidence range for $\alpha\left(f^{\sim}{ }^{-\alpha}\right)$ of $0.62(+0.36$, $-0.24)$ for $\mathrm{N}_{\mathrm{H}} \leqslant 3 \quad 10^{20}$ atoms $\mathrm{cm}^{-2}$. Higher column densities for OSOs are not anticipated, but if $\mathrm{N}_{\mathrm{H}}$ were $\sim 10^{22}$ atoms $\mathrm{cm}^{-2}$, then $\alpha \geqslant 0.75$. Although, we find no evidence for the soft energy excesses typically seen in BL Lac-type objects, and our spectrum is similar to those exhibited by Seyfert galaxies, the spectrum is soft enough that, if these objects are typical of the higher redshift more radioquiet QSOs, then QSOs may perhaps be excluded as being the dominant origin of the diffuse X-ray background. (See De Zotti et. al., 1982, Ap. J., 253, 47).

Fred Bruhweiler (Computer Sciences Corporation) and I have observed the BL Lac-type objects OJ 287, OQ 530, 3C 371 and ON 325 with the IUE satellite. This has been done in conjunction with collaborators who have made nearly-simultaneous observations at visual, infrared and radio wavelengths. We have applied synchrotron selfCompton emission models to the data. Two relevant parameters for each source, the self-absorption frequency of the synchrotron source and the source size at this frequency (or source variability time scale), are not known. The smallest synchrotron components, which are the ones producing the most Compton radiation, may be self-absorbed at radio frequencies above those at which the sources are mapped. The use of reported radio source sizes may thus lead to erroneous conclusions. We find, however, that our higher energy spectral measurements provide sufficient evidence alone to show that either relativistic beaming is present in these sources, or that a more complex model involving an electron reacceleration mechanism is required.

G.A. Gurzadyan and V.G. Gurzadyan: Two facts concerning the behaviour of resonance ultraviolet doublet of ionized magnesium $2800 \mathrm{MgII}$ in emission in the spectra of quasars (QSOS) and active galactic nuclei attract one's attention. The first one is attributed to the ratio of observed flux in MgII doublet to the flux in hydrogen $L_{\alpha}$-line: $F\left(\operatorname{MgII} / F\left(L_{\alpha}\right)\right) \equiv Q$. For usual stars these lines are generated 
in the stellar chromospheres and always $Q>1$, varying in a band $2-20$; for instance, $Q$ (Sun) $=4.5$. However for QSOs, as a rule, $Q<1$; for investigated QSOS $Q \simeq 0.2$ The second fact concerns the width of MgII and HB emission lines. For QSOs their width corresponds to very high velocities $\sim 5000 \mathrm{~km} \mathrm{~s}^{-1}$. Here the following problem is examined: is it possible on the basis of these two extraordinary facts peculiar only to QSOs and Seyfert galaxies to decipher their internal structure and to throw light on the nature of emission regions? We point out that the observational data can be explained by a presence of star-mass ionized cloud system with Maxwellian velocity distribution. A model of generation of these clouds is proposed.

A. Pedlar: NGC 1275 (Perseus A, 3C84) is surrounded by a series of Ha filaments which Cowie et al. (MNRAS 191,399) believe to be due to cooling accretion flows from hot $\left(10^{7} \mathrm{~K}\right) \mathrm{X}$-ray emitting gas in the Perseus cluster. We have mapped the $\lambda 73 \mathrm{~cm}$ radio continuum emission from NGC 1275 using MERLIN with a resolution of 1 arcsec. The resulting structure shows a $5 \mathrm{kpc}$ radio jet emanating from the nucleus in $\mathrm{PA} \sim 160^{\circ}$. This aligns approximately with the milliarcsecond jet in the nucleus ( $\mathrm{PA} \sim 170^{\circ}$ ). Of particular interest is a filament in PA $\sim 170^{\circ}$ which aligns precisely with the VLBI jet, and is also unique in that it shows by far the largest velocity gradient in any of the filaments and extends $\sim 1$ arc min from the nucleus. We attribute this to the action of a beam which originates in the nucleus and consider the ionised gas to have been entrained into the beam and transported to the outer part of the galaxy before it cools to $\sim 10^{4} \mathrm{~K}$ and emits $\mathrm{H} \alpha$ lines. We therefore feel that this particular filament is not caused by a cooling accretion flow, but is associated with ejection from the nucleus. Consequently optical comparisons between this filament and others in the halo would be useful to confirm our hypothesis.

A full account of this project is about to be submitted to MNRAS.

The work was done in collaboration with $R$. Booler and $R$. Davies.

E. Valtaoja: A. 15.7 minute periodic flux variation wás noticed in oJ 287 in April 1981 at $37 \mathrm{GHz}$. The source has been subsequently monitored for 142 hours att $22 \mathrm{GHz}$ and $37 \mathrm{GHz}$ as well as in optical photometry. The 15.7 minute period was discovered at two simultaneous photometric observations in February 1982, and the same period was also seen at $22 \mathrm{GHz}$ at the same time. The optical variations were observed in phase with each other at both observatories, but the radio variations were out of phase by about one third of the phase. The peak-to-peak amplitude of the variation is about $5 \%$ of the total intensity, and the period is $943 \pm 10$ seconds. The results support a spinar model for OJ 287 .

The investigation has been carried out in collaboration with H. Lehto, P. Terrikorpi, S. Haarala, T. Korhonen, M. Valtonen, H. Teräsranta, E. Salonen, S. Urpo, M. Tiuri and V. Piirola.

G. Pastoriza: Metallicity of $8 \mathrm{E}$ and so galaxies as well as that of red globulars of the IMC and SMC were obtained by means of DDO integrated photometry calibrated with galactic globular clusters (Bica and Pastoriza, 1982). A correction was obtained in order to reduce the colors of the galaxies to zero redshift. The relation metallicity vs $M_{V}$ for the galaxies is analyzed (adding to our sample the observations of McClure and Van den Bergh, 1968 and Faber, 1973). For the Magellanic Clouds we found metallicity ranging from intermediate to poor. The investigations has been carried out in cooperation with H. A. Dottori and E. L. D. Bica.

Ch. J. Peterson: The Rubin et al. (1976, Astron. J., 81, 687, 691) study of a distant sample of SC I galaxies produced a solution for the motion of the Local Group that was (and still is) at variance both with several other determinations which have used other selections of galaxies and with the motion deduced from the anisotropy of the cosmic background radiation. The weakest part of their study was reliance (in part) on photographic magnitudes (which they corrected) from the Zwicky et al. (1961-1968) Catalogue of Galaxies and Clusters of Galaxies. We now have Kitt Peak photoelectric photometry of their galaxies and reanalysis for the motion by the method of Jackson (1982, Ph. D. thesis, University of California - Santa Cruz) shows that use of the more reliable photoelectrically 
derived magnitudes does not remove the differences between the motion vector from the Rubin et al. galaxies ( $V=671+195 \mathrm{~km} \mathrm{~s}^{-1}$ toward $\mathrm{I}=200^{\circ}, \mathrm{b}=-12^{\circ}$ ) and the motion derived from closer samples of galaxies (e.g. de Vaucouleurs et al. 1982, Astrophys. J., 248, 395, 408) and that derived from the cosmic background radiation (Boughn et al. 1981, Astrophys. J. (Letters), 243, L 113). The new observational data remove any last doubt that the Rubin et al. motion is an artifact of systematic errors in the Zwicky magnitudes. Jackson's work further eliminates as sources of error any bias in the Rubin et al. selection of galaxies in terms of either apparent or absolute magnitudes, use of a simple cosecant law for the galactic absorption, or the methodology of analysis. We suggest that the differences in derived motions may merely reflect the complex flow patterns and inhomogeneities of the galaxy distribution in the local region of the present day universe.

This work has been supported by the U.S. National Science Foundation.

\section{Report of Working Groups}

\section{THE MAGELIANIC CLOUDS}

The Working Group met on 25 August. Dr M. W. Feast was re-elected Chairman.

The following papers were read:

D. Lynden-Bell: Which Way are MC Going?

R. J. Cohen and R. Davies: The Magellanic Stream

M. T. Brück: The SMC Halo

T. Lloyd Evans: $C$ and MS stars in the MC

M. F. Walker: Santa Cruz MC Programmes

M. S. Bessell: Red Variables in MC

J. Isserstedt: Star Formation in MC (film)

Ch. Fehrenbach: MC Catalogues

A. F. J. Moffat: The Core of 30 Doradus

A. F. J. Moffat: WR stars in MC

M. Kontizas: Kron 3 and NGC 152

M. Kontizas: Dynamics of SMC Clusters

N. R. Walborn: Supergiant Planetaries in MC

$K$. Nandy: U-V extinction in SMC

P. Dubois: The Thickness of the SMC

B. Y. Mills: MC SN Remnants

D. Crampton and J. B. Hutchings: MC XR Binaries

C. Chiosi: Star Formation in MC

\section{GALAXY PHOTOMETRY AND SPECTROPHOTOMETRY}

The Group convened in the afternoon of August 24 jointly with the $W$. G. on Internal Motions. The Chairman of the W. G. acted as chairman of the session, which started with the business. It was unanimously agreed that the W. G. should continue to exist. It was also decided to elect the $W$. G. Chairman for the next triennium by consulting all members by mail. A drafted resolution aiming to simplify the notation of units commonly used in surface photometry work was presented by de Vaucouleurs and Capaccioli. They also proposed to constitute a task force or consortium for a careful photometric mapping of the Virgo Cluster galaxies.

The following papers were presented:

S. Okamura: Recent Work on Galaxy Photometry in Japan

J.-L. Nieto: Recent Results from High-Resolution Observations of Central Regions of Galaxies

Ch. Peterson: Systematic Errors in the Magnitudes of Zwicky Catalogue Galaxies

H. Lorenz: Image Processing by Means of A Digital Filter Technique

M. Capaccioli: Some Properties of Early-Type Galaxies Inferred from Photometric Mapping 
ABSTRACTS OF PAPERS:

S. Okamura: The present status of galaxy photometry in Japan was briefly reviewed. The major programs carried out recently or in progress now at several institutions were referred to, and the results of some recent works were reported. Preliminary results were presented of the surface photometry program by the Tokyo group in which they measured the two-dimensional luminosity distribution of some 260 galaxies of various morphological types in Virgo and Ursa Major regions in the $\mathrm{V}$-band on the basis of the photographic plates taken with the 105-cm Schmidt at Kiso Branch of the Tokyo Observatory. They could support the consortium proposed at the meeting by Capaccioli and de Vaucouleurs by extending this project.

J. L. Nieto: Two sets of observations are presented which were made at the prime focus of the CFH telescope. The resolution of these data is very good, which confirms the high quality of the images obtainable at this site. 1 - NGC 6251 exhibits a very narrow dust lane almost perpendicular to the major axis of the optical image. The dust lane is parallel to the radio jet. This suggests a random orientation of the nuclei of galaxies with respect to their main bodies, represented for instance by the rotation axis of the dust and the gas. 2 - NGC 3379 was observed with resolutions between 0.5 and 3 arc-seconds. Adding all the relevant data published in the literature to these new data allowed the building of the high resolution profile of this galaxy. Some evidences for the existence of two populations as well as a peak of light in the center of this galaxy are presented.

Ch. Peterson: From a comparison of photoelectrically derived total magnitudes of a sample of $119 \mathrm{Sc}$ galaxies with the photographic magnitudes of the Zwicky et al. Catalogue, we find there exists in the photographic data both a scale error and a correction based on the average surface brightness $\mathrm{m}^{\prime}$ :

$$
\mathrm{B}(\mathrm{pe})=\mathrm{A}+(1.079 \pm 0.008) \mathrm{m}(\mathrm{pg}, \mathrm{Zw})-(0.464 \pm 0.003) \mathrm{m}^{\prime}(\mathrm{pg}, \mathrm{Zw})
$$

These coefficients are essentially the same as found by de Vaucouleurs and Pence (1979) from a study of Virgo region galaxies. The zero point $A$ is essentially the same for the galaxies of the catalogue volumes I-V. Volume VI differs by 0.3 magnitudes. Prior investigators, on the other hand, have suggested that Volume I systematically differs from the remaining Volumes. It is suggested that this apparent conflict is due to a need to correct Zwicky data also for galaxy type.

H. Lorenz: On the basis of the HAAR transformation a processing of astronomical images is described which separates the noise optimally and reduces the redundancy of the data up to 100. The system fits its own bandwidth to the incoming signal and steeres a two-dimensional KALMAM-FILTER: The objects in the field are picked up in the domain of the HAAR-tranform. Astronomically relevant parameters are derived from the expansion of the intensity distribution into potential moments.

A measuring system is planned in cooperation with VEB Carl-Zeiss Jena. This system will include software packages for different applications and it will ensure mechanical accuracy and stability (air bearings, laser metering).

M. Capaccioli: A brief account is given of the work done at Padova-Asiago in the field of galaxy surface photometry, with emphasis to the study of the properties of early-type galaxies.

1 - A photometric and geometrical investigation of ten galaxies in Virgo is presented which, together with previously published studies, stresses the need for further, more accurate work.

2 - From the analysis of the available luminosity profiles for $E$ galaxies we find a systematic behaviour of the residuels with respect to the $\mathrm{rl} / 4$ law.

3 - We have re-discussed the luminosity law which represents the bulges of so's, finding it steeper than before thought.

\section{INTERNAL MOTIONS IN GALAXIES}

A short business session was held at the joint meeting of the Working Group on Internal Motions in Galaxies with the Working Group on Galaxy Photometry and Spectrophotometry. It was agreed that the Working Group on Internal Motions in Galaxies served a very useful purpose in circulating preprint information. In the 
absence of alternative nominations S. T. Gottesman agreed to serve for another 3 years as chairman of that Working Group. However, he noted that he wished this to be his last term in that position.

A total of 9 papers concerned with internal motions in galaxies were read before the two Working Groups. S. Simkin reported on the alignment of the axes of radio sources with the rotation axes of galaxies. She finds that for high luminosity sources the axes are closely aligned. S. Simkin also read a paper by Pronik reporting variability, over a period of 10 years, in the $H \beta$ flux and forbidden line strengths observed in the galaxies NGC 1275 and NGC 3227. Pronik and Merculova, as reported by Simkin, find that over a shorter period of 4 days the HB flux of NGC 1275 may vary by a factor of 2. Terzian reported on the Arecibo program to measure the HI properties of binary and multiple galaxies. A tendency has been found for the total mass to light ratios to be similar within pairs. Brinks showed his film on the kinematics and structure of the atomic hydrogen in M 31. He noted several features of interest including the non-circular motions of the inner spiral structure. U. J. Schwarz reported on a Westerbork study of HI in the peculiar system NGC 3718/3729. Many of the properties can be explained by a warped edge-on ring. J. M. van der Hulst presented new VLA observations of the barred spirals NGC 1097 and NGC 1365. For the latter he finds no gas in the central region, whereas NGC 1097 reveals HI in the bar zone. However, its structure is complicated by a possible interaction with a nearby companion. S.T. Gottesman reported on the VLA observations of the HI in the barred systems NGC 3992 and NGC 4731. The barred central region of NGC 3992 showed little or no HI while the bar of NGC 4731 appears quite gas rich. The final paper was read by G. L. Pilbratt on CO observations of M5I obtained with the Onsala telescope. These data show little contrast between the arm and inter arm regions. However, further observations are in progress.

\section{SPACE SCHMIDT SURVEYS}

This Working Group met on the afternoon of August 25. The session began with a brief business meeting, during which it was decided that the current chairman, $K$. Henize, and vice-chairman, F. Bertola, would continue in their offices for the next 3 years.

The scientific session was initiated by H.J Smith, who reviewed the instrument concepts and scientific importance of the space Schmidt program and reviewed the status of the multi-national proposal for a Space Schmidt Telescope (SST) which is coordinated by $\mathrm{J}$. Wray at the University of Texas.

R. Barbon reviewed the extensive studies taking place at Asiago/Padova, in cooperation with Aeritalia Corporation, concerning the design of the main structure of the SST. (See abstract).

H. Heckathorn described progress at the Naval Research Laboratory (NRL) on the development of large-format high-resolution UV electrographic detectors for the SST (See abstract).

In a separate paper, Heckathorn described follow-up studies of very hot, faint stars discovered by the NRL S201 UV telescope carried on the Apollo 16 and Skylab missions.

In the first of three papers on telescopes and/or techniques related to the objectives of the SST, T. Gull reviewed the characteristics and scientific objectives of the UV telescopes to be carried on the NASA OSS-3 payload.

H. Lorenz described a method for producing spectra of very faint objects using a multi-slit field spectrograph with the 6-meter telescope. (See abstract).

$K$. Kodaira described the $60-\mathrm{cm} \mathrm{f} / 4 \mathrm{UV}$ reflector, VVT, which is under study for flight on the Japanese Ultraviolet-Telescope Satellite (UVSAT).

ABSTRACTS OF PAPERS:

R. Barbon: Most of the efforts in Italy have been so far devoted to a feasibility study of the telescope main frame made by AERITALIA, a well known space contractor. Although the study resulted positive toward the fabrication 
and operation of the instrument, the total cost estimate for the hardware to be built in Italy came out much higher than planned. A new approach, which makes use of graphite-epoxy, looks more promising since this material, with an extremely low coefficient of thermal expansion, leads to a structure with lower weight and passive thermal control only, thus reducing the cost considerably. AERITALIA has been asked to provide an engineering verification of the new design and a cost estimate. In the mean time the Italian groups involved in this project have pointed out the areas of interest for the research programs to be accomplished during the first flights as follows. I. Spiral structure in nearby galaxies: theories vs. observations. 2. Progenitors of type I SNe. 3. Young stellar population in elliptical galaxies. Morphology of diffuse nebulae.

H. M. Heckathorn and G. R. Carruthers: The Naval Research Laboratory has a program to develop electrographic detectors for use in a variety of space astronomy and other space science applications. Of particular interest to this Working Group is our continuing development of UV-sensitive electrographic detectors having large-diameter, semitransparent photocathodes which are designed for use at the focal surface of a large auxiliary optical system such as the folded allreflecting Schmidt. Our presentation will describe technical developments which have taken place since the publication by NASA of the "Spacelab Wide Angle Telescope Working Group Report" in 1979. Four specific developments will be discussed in detail.

(1) Successful fabrication of two large diameter (177-mm), thin (1-mm), curved (2000-mm radius), and UV-transmitting (calcium fluoride) faceplates.

(2) Successful design of a permanent magnet focussing assembly of the size and field uniformity required by the space schmidt detector.

(3) Successful modification of our prototype large-format (70 \% scale) detector to allow for (1) continuous vacuum pumping, with a demountable but sealed tube structure, and (2) the imposition of a thin, electron-permeable barrier membrane (Iocated between the photocathode and the recording emulsion) which is both optically opaque and impervious to the products of film outgassing.

(4) Continued development of medium- and large-format electrographic detectors sensitive in the mid-UV spectral region $(1700-3200 \AA)$ which make use of "solar-blind" cesium telluride photocathodes coupled with UV-grade fused silica faceplates.

At this point, we feel that technical feasibility has been demonstrated for all aspects of the far-UV large-format electrographic detector for the space Schmidt save one: verifying that the thin faceplate can survive the vibration and acoustic environment of a shuttle launch. We intend to conduct vibration tests on one of the two calcium fluoride faceplates, in an evacuated housing and mounted on a prototrpe image tube structure as nianned for the Snace Schmidt. The other faceplate will be used (with a cesium iodide semitransparent photocathode) in a laboratory full-scale prototype detector, when and if funds for the construction of this detector (and procurement of the PFMA) become available.

V. Afanasiev, I. Karachentsev, S. Dodonov, I. Terebish, H. Lorenz: A "multi slit method" is described which improves by means of night sky masking the limiting magnitude. The spectrograph is based on focal reducer optical arrangements. A limiting magnitude of $22.5 \mathrm{mag}$ in $B$ has been achieved for spectra with a reciprocal linear aispersion of $650 \mathrm{~A} / \mathrm{mm}$. The preliminary results indicate a substantional flattening of the $\log \mathrm{M} / \mathrm{log} \mathrm{S}$ diagram for quasars at magnitudes of $21^{m}-22^{m}$. 
COMMISSION 29: STELLAR SPECTRA (SPECTRES STELLAIRES)

Report of the Business and Scientific Meetings, 18-25 August 1982

PRESIDENT: W. Bonsack

SECRETARY: C. Jaschek

\section{Business Meeting}

The triennial business meeting of the Commission was opened by the President, who first gave a report on the activities of the Commission since the last meeting. The principal activities consisted of sponsorship of symposia and colloquia, of working groups on specialized topics, and of the International Register of Stellar Spectroscopists. The Commission participated in six IAU-sponsored meetings in 1979-82, and the Organizing Committee has approved sponsorship of two more in the near future. The IRSS is a list of stellar spectroscopists giving names, addresses, and research specialties, without regard to IAU membership. It is edited by $W$. Bonsack and A. Slettebak, and is co-sponsored by Commission 45 . A current computer file of the contents of the IRSS is maintained at the University of Hawail. Two editions have been printed and distributed without charge to all persons listed and to 150 institutions; the current file contains 260 names.

The President also reported that the members of the Commission currently number 185, with approximately 15 new members to be added. Four members of the Commission have died since 1979, Including the President of the IAU, Prof. Bappu. There have been no resignations.

The future of the IRSS was discussed, and it was voted to continue the sponsorship by the Commission; Bonsack and slettebak are willing to continue as editors.

Reports on the Working Groups sponsored by the Commission were given by the Chairs of the WG's.

WG on Be stars: The report was presented in written form by M. Jaschek. The WG organized an IAU Symposium on Be Stars (no. 98, Munich, FRG), and pub11shes a newsletter. A scientific meeting was planned for the General Assembly. The WG is cosponsored by Comm. 45.

WG on Ap stars: The report was presented in oral and written form by $C$. $R$. Cowley. The WG particlpated in organizing the 23rd Liege Internationa1 Co1loquium, and has joined with an Informal European group in publishing a newsletter. A paper glving recommendations for abundance determinations in Ap stars is being prepared by Adelman and Cowley. A scientific meeting has been planned for the General Assembly. The WG is cosponsored by Comm. 45.

WG on Standard Stars: This WG was recommended at the 1979 business meeting, and was organized by L. Pasinett1, with the collaboration of a provistonal committee. The WG w111 deal with the problem of identifying "normal" standard stars, and providing standard reference data, for various problems in stellar astronomy. A scientific meeting was organized for the General Assembly, at which the need for standard stars and data in various fields were summarized. The WG 1 s cosponsored by Commissions 30 and 45 .

The president asked the Commission if it wished to formally sponsor the WG on 
Standard Stars. By a show of hands, the Commission voted to sponsor the WG.

C. Jaschek proposed that the Commission approve the proposal of A. Batten to co-sponsor a Symposium on Calibration in Astronomy. Garrison and Underhil1 suggested that the topic is too broad, and should be restricted severely. It was agreed to refer the question to the WG on Standard Stars for recommendation (Note: after a further urgent request from $\mathrm{Dr}$. Batten for a decision by the Commission, the 0.C. declined to sponsor the meeting, on the grounds stated above).

The election of officers and the Organizing Committee of the Commission was carried out. Bonsack presented the selection of the 0.C. for the new officers: President, Dr. Jun Jugaku; Vice President, Dr. Giusa Cayrel-de-Strobe1. The Commission confirmed this selection. A slate of 14 candidates for the 0.C. was presented, which included six from the previous $0 . C$. who were willing to continue, and eight additional who had been selected to give geographic balance, and who had indicated willingness to serve if elected. The president called for further nominations, but none were offered. Voting was by paper ballot, each member present voting for up to eight candidates for the eight offices; the eight with the most votes were declared elected. Those elected were Y. Andrillat, P. Conti, C. Cowley, C. Jaschek, H. Levato, M. Smith, R. Viotti, and S. Wolff.

The President next reported on Colloquia and Symposia which had been proposed for sponsorship or co-sponsorship. The titles of the meetings with their proposers are "Observational Tests of Stellar Evolution Theory" (A. Maeder); "Origins of Non-radiative Heating/Momentum in Hot Stars" (A. Underhil1); "MK Spectral Classification: Criteria and Applications" (R.F. Garrison); "Structure and Evolution of the Magellanic Clouds" (S. v.d. Bergh, K.S. de Boer); and "Late-Type Chemically-Peculiar Stars" (P.C. Keenan); "Luminous Stars, Assoclations, and Galaxies" (P.S. Conti). The first two of these meetings had already been approved for sponsorship by the OC. Drs. Garrison and Underhill described thelr proposed meetings. At the request of $\mathrm{Dr}$. Keenan, his proposed meeting was discussed, including the invitation conveyed by Mrs. Harmer to hold it at the Royal Greenwich observatory. It was recommended that the meeting topic should be broad enough to include all chemically peculiar stars of late type, which would attract more participants than could be accommodated by RGO. C. Jaschek proposed that the meeting be held at Strasbourg. Bonsack agreed to convey these comments to Keenan and Harmer.

\section{Scientific Meetings}

SYSTEMATIC EFFECTS IN ABUNDANCE DETERMINATIONS FOR METAL-POOR STARS (Joint meeting of Commissions 25, 29, 37, 45). The meeting was intended to explore the technical problems which have led to significant discrepancies in the abundances obtained for the more metal-rich globular clusters by different techniques. Invited papers were presented by M.S. Bessell on "Low-resolution and Photometric Abundance Studies"; by R.C. Peterson on "High-resolution Abundance Studies", and by B. Gustafsson on "Model Atmospheres and Spectrum Synthes1s". Short papers were contributed by A. Underhill, M. Spite, M. Grenon, R.P. Kraft, and R. Rutten. A summary was given by G. Cayre1. The meeting was organized by a committee chaired by $W$. Bonsack; the invited papers will be published in the Publications of the Astronomical Society of the Pacific.

SCIENTIFIC MEETING OF THE WORKING GROUP ON Ap STARS (Joint meeting of Commissions 29 and 45). The scientific meeting was orgainzed and chaired by C. Cowley, and consisted of short review papers on Digital Data Processing, Metal-Weak $A$ and B Stars, High-Velocity A and B Stars, CP Stars in Clusters, Turbulence and Diffusion Theory, Microturbulence and Abundances, Magnetic Fields, Abundance Patches, and Rapid Var-iations. The papers emphasized the progress in the field since the Liege Symposium in 1981. In addition to the scientific program, a new Organizing 
Committee was nominated and elected by those present. C. Cowley was re-elected chairman.

MEETING OF THE WORKING GROUP ON STANDARD STARS (Joint Meeting of Commissions 29, 30, and 45). The program began with a business session, in order to discuss the program of the WG, and to elect an Organizing Committee. L. Pasinetti chaired the meeting, and gave an introduction listing possible items for the program, including (1) revision of existing lists of standard stars, (2) study of standard stars with modern techniques and criteria, (3) the spectral type of the sun and solar analogues, and (4) distribution of standard stars around the sky. The invited papers reviewed the current situation in some of the above respects: standard velocity stars (A.H. Batten), standard stars for polarization (M. Breger), 1dentiflcations and equivalent width measurements from photographic plates (C. Moross1), and UV catalogues and standards (K.A. van der Hucht). A paper on the mid-UV spectrum of Procyon was contributed by F. Castelli, et al. The Organizing Committee elected for 1982-85 is A.H. Batten, M. Breger, R. Garrison, M. Gerbaldi, I.N. Glushneva, M. Hack, C. Jaschek, L.E. PasinettI (chair), F. SpIte, and K.A. van der Hucht.

MEETING OF THE WORKING GROUP ON Be STARS (Joint Meeting of Commissions 29 and 45). Proceedings will be reported by Commission 45 .

ATOMIC AND MOLECULAR DATA IN STELLAR SPECTRA (Joint Meeting of Commissions 14, 29, and 36). Proceedings will be reported by Commission 14 .

\section{Toint Discussions}

Commission 29 participated in two Joint Discussions held at the General Assembly: JD II, "Evolution in 01d Stellar Populations in Galaxies," and JD VII, "Mass Loss Phenomena". 
Compte Rendu de séances, 21 Août 1982

PRESIDENT: M. Duf 1 ot

SECRETAIRE: J, Andersen

\section{SEANCE ADMINISTRATIVE}

1. Bureau $1982-1985$ de 1a Commission

Les propositions suivantes du Comité d'Organisation sont approuvées à l'unanimité des membres présents :

Président : A.G. Davis Philip

Vice Président : J. Andersen

Membres du Comité d'Organisation : M. Duflot, D.P. Hube, E. Maurice, M. Mayor, J. Sahade

\section{Motions de soutien}

La Commission a décidé de participer avec la commission 29 à la création du Working Group sur les étoiles standards proposé par la Commission 45 , et de nommer A.H. Batten comme représentant.

D'autre part la Commission a décidé de soutenir la demande de A.H. Batten pour un Symposium sur la calibration en astronomie qui se tiendrait en 1984 à Como en Italie.

Les deux motions suivantes ont donc été approuvées

- Commission 30 cosponsors the Working Group on standard stars created by Commission 45 .

- Commission 30 supports the proposal that a Symposium on "Calibration in Astronomy" be held in 1984.

\section{Futur Symposium sur les Vitesses Radiales}

A.G.D. Philip a proposé un Symposium sur les Vitesses Radiales qui pourrait avoir lieu en automne 1984 (Albany). La Commission donne son accord pour que A.G.D. Philip continue à préparer ce symposium et fasse la demande officielle auprès du Comité Exécutif de 1'UAI.

\section{Etoiles de vitesse radiale standard}

Après un rapport présenté par A.H. Batten, la Commission a approuvé les décisions suivantes :

(a) The star HD 184467 is now known to be a 2 sp binary ( $\sim 480 \mathrm{~d}$ ).

- The star HD 35410 is probably a l sp binary.

- The star HD 14969 is a binary.

These stars should not be used as standards and deleced from the list of standard stars. 
(b) The following stars should be considered as secondary standards, suitable for use only at low dispersion :

\begin{tabular}{|c|c|}
\hline Bright Stars & Faint Stars \\
\hline $\begin{array}{r}36673 \\
84441 \\
102870 \\
186791 \\
206778\end{array}$ & $\begin{array}{r}3765 \\
9138 \\
26162 \\
29587 \\
51250 \\
65583 \\
92588 \\
103095 \\
115521 \\
126053 \\
144579 \\
145001 \\
154417 \\
187691 \\
223311\end{array}$ \\
\hline
\end{tabular}

(Some of them may be variable and some may have velocities different from the adopted values).

Rappelons qu'en 1958 (Transactions UAI 10), la sous-commission 30a "recommended that the stars $\alpha$ Per, HD 20902, and $\alpha$ Car, HD 45348, should be dropped from the list of standard velocity stars".

\section{Working Group sur les vitesses radiales moyennes}

C. Jaschek a souligné l'importance de la demande faite par la communauté astronomique d'un catalogue de vitesses radiales moyennes.

Mne M. Barbier, présidente du WG, a communiqué les conclusions du groupe qui s'est attaché à trouver une solution qui permette d'obtenir les VR moyennes au moyen d'un ordinateur. Cette solution est discutée.

Afin de pouvoir prendre une décision définitive, les deux premières heures du catalogue seront réalisées, le plus rapidement possible, sur les bases proposées, et soumises au Comité d'Organisation de la Commission. Mme M. Barbier demande le maintien du WG actuel (M. Barbier, A.H. Batten, M. Duflot, D. Evans, R. Griffin) et $J$. Andersen accepte de s'y joindre.

\section{SEANCE SCIENTIFIQUE}

During the scientific session, the following papers were given:

J. Dommanget : Informations et problèmes relatifs à l'observation des vitesses radiales des étoiles doubles visuelles.

The necessity was stressed to obtain radial velocity observations in order to define orientations of the orbital planes and any possible systematic in these.

Ch. Fehrenbach : Le prisme objectif de $60 \mathrm{~cm}$. Résultats actuels.

La mesure des clichés obtenus au prisme objectif à champ normal de $60 \mathrm{~cm}$ est faite par enregistrement digitalisé de pointés photométriques et par corrélation. La méthode a été améliorée par la mise en mémoire dans 1 'ordinateur de 6 spectres 
étalons. La précision des mesures est de $4 \mathrm{~km} \mathrm{~s}^{-1}$ pour une moyenne de trois clichés et ceci pratiquement quel que soit le type spectral, alors que la dispersion n'est que de $200 \mathrm{~A} \mathrm{~mm}^{-1}$. On obtient sur plaque IIIaJ des étoiles de magnitude 12 avec deux poses de $40 \mathrm{~m}$ (méthode de retournement).

\section{W.I. Beavers : Radial velocity program and future plans at the Erwin Fick observatory.}

Exploiting the advantages of a dedicated coudé instrument, experiments are made with special masks for various types of objects. Observations are concentrated on bright stars, including checks of long term stability of standard and other stars.

M. Mayor : Reports on CORAVEL measurements.

A brief report was given on the capabilities of CORAVEL to supply metal abundances and rotational velocities as a by-product, and of the large-scale programmes undertaken, especially those with CORAVEL II in the southern hemisphere (LMC + SMC supergiants and cepheids, $\omega$ Cen, 47 tuc, Pop.II stars).

D.W. Latham : The digital stellar speedometer at the F.I. Whipple Observatory.

Recording one order of a cross-dispersed echelle spectrum with a photon counting Reticon detector in one of several similar instruments designed for determination of galaxy redshifts, stellar radial velocities are determined with good precision by software correlation with a library of standard spectra. Limiting magnitude is about 17.5 with the MMT.

\section{J. Andersen : Radial Velocities of Bright Stars ; Future Instrument Developments.}

Through a collaboration on coudé and CORAVEL observations from ESO, La Silla, only 9 observations now remain to complete all HR stars, and observations are started on some $4000 \mathrm{~F}$ stars of known age and metal abundance. The gross inadequacy of the existing set of standard stars to check observations of B, $A$, and $F$ stars of different rotations was stressed.

Combining existing optical, electronic, and software advances (Mayor, Latham) it should be possible to build instruments allowing either rapid, on-line results on bright stars with a small telescope, or reaching $B=18^{\mathrm{m}}-19^{\mathrm{m}}$ on a $4 \mathrm{~m}$ telescope (old SMC/LMC and dwarf galaxy giants, globular clusters, .....).

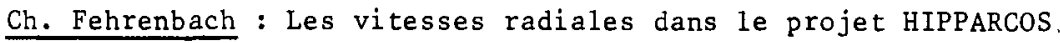

Radial velocities of the northern early-type HIPPARCos stars will be determined using the objective prism-technique (see above) in fields of sufficient star density, and coudé observations otherwise.

F. Feke 1 : Standard Stars Observed at the McDonald Observatory.

Results for some bright A-F stars found to be constant over several years of use as standards in spectroscopic binary programmes were given. 
D. Dravins : Astrometric Determination of Absolute Heliocentric Radial Velocities in Moving Clusters.

It is proposed to use HIPPARCOS determinations of proper motions and convergent points for the Hyades and Ursa Major to compute absolute radial velocities of individual stars to about $400 \mathrm{~m} / \mathrm{s}$. Comparison with the direct measurements will yield information on other sources of systematic line shifts (convection, relativistic redshift, ....). 
COMMISS ION 31: TIME

(L'HEURE)

Report of Meetings, 19, 20,23 August 1982

PRES IDENT : S. I i j ima

SECRETARY: D.D. MCCARTHY

General

Prior to Commission meetings, a short session of the Organizing Carmittee was held on the afternoon of 17 August 1982 in which items and proposals to be submitted for consideration by the Cormission were reviewed.

Twenty-five members of Commission 31, four future members, three consultants, and four invited participants attended Comission 31 meetings.

\section{August 1982}

In a joint meeting of Commissions 19 and 31 the progress of Project MERIT was reviewed. Talks by G. Wilkins I.I. Mueller, and M. Feissel are summarized in the report of IAU Camission 19.

These discussions were followed by presentations by each of the principal coordinators of the project.

K. Yokoyama pointed out the Short Campaign had led to an improvement in the results from optical astrametry and that a further improvement could be expected during the Main Campaign as a result of the use of more accurate star catalogs for the astrolabes and PZTs and better corrections for nutation and tidal effects. He hopes to determine short-period variations from the observations at the better stations.

C. Oesterwinter reported that data fram the Doppler tracking of satellites had been analyzed in an attempt to detect plate motions; the values found for the Australian and Pacific plates appeared to be significant and in general agreement with geological studies. The lack of proper information about local changes in the positions of the antennae had hindered the analyses. In reply to a question B. Guinot stated that a new MEDOC II system is being developed; it will include four new French stations and should come into operation during the Ma in Campaign.

B. Schultz reviewed the current status of the satellite laser ranging stations that supplied data to the analysis centers at Greenbelt and Austin in the USA. For many stations the precision is about $\pm 10 \mathrm{~cm}$ and for one or two it is $+2-3 \mathrm{~cm}$. There is a very noticeable weekend effect in the frequency of observations; a better balance between day and night observations will also be required if reliable determinations of short-period variations are to be made. More effort must be made to reduce both systematic and random errors. B. Guinot cammented that the monthly reports fram the University of Texas are very useful.

O. Calame said that lunar laser ranging contribution to MERIT was carried out in the $\mathrm{f} r$ amework of the EROLD campaign, but was 1 imited to the determination of UT since data were only available from the McDonald Observatory. She expressed the hope that LLR data would also be available from Grasse, Wettzell and Orroral during the Main Campaign, and she presented solutions for the annual and semi-annual terms in UT fram MeDonald data for the period 1971-1981. 
W.J. Klepezynski spoke mainly about the connected-element radio interfercmeter at Green Bank since this was the only CERI system in regular use for the determination of Earth-rotation parameters. Some results had been obtained fram Cambridge, England, during the Short Campaign and he hoped that some time would be available on the Very Large Array at Socorro during the Main Campaign. The Green Bank system is being extended to allow the determination of all three parameters, and a water-vapor radiameter is being installed in order to reduce the errors due to the troposphere. In response to a question he said that it might be possible to obtain a solution with a precision of $+0.5 \mathrm{~ms}$ at an interval of 12 hours.

D.S. Robertson showed results fram VLBI and claimed that UT could be obtained with a precision of the order of $\pm 0.1 \mathrm{~ms}$ fram 24 hours of observation in spite of bad weather and bad ionospheric conditions. The POLARIS network in the USA requires one more element in order to obtain all three parameters; observations will be made every five days. In response to a question he said that this network when used with another element at Wettzell, Germany, should give results with precisions of $\pm 0.1 \mathrm{~ms}$ and $\pm 5 \mathrm{~cm}$ fram 24 hours of observation.

In the ensuing general discussion G. Wilkins stated that the third and final draft of the MERIT recommendations on standards should be available during the autumn and that recommendations on the monumentation of sites would be prepared since this will be very important in the establistment of the new conventional terrestrial reference frame.

The 47 voting participants then unanimously endorsed the following two resolutions .

The International Astronomical Union

taking note of the success of the MERIT short campaign, and

recognizing that the results to be obtained during the MERIT main

campaign will be of short and long-term benefit for the studies of the planet Earth

endorses the following two resolutions adopted by the International Association of Geodesy (IAG), at its General Meeting at Tokyo (May 1982):

l'Uni on Astronomique Internationale

prenant note du succes de la campagne courte du programe MERIT

et

reconnaissant que les résultats à obtenir au cours de la campagne principale de MERIT apporteront des contributions à long et à court termes pour les etudes de la planète Terre

soucrit aux deux résolutions suivantes adoptées par l'Association Internationale de Géodésie (AIG) lors de sa réunion générale de Tokyo (Mai 1982):

\section{AIG Resolution 1}

The International Association of Geodesy noting that the results to be obtained during the MERIT main campaign will be of long-term benefit to geodesy and its applications

strongly endorses the proposals of the COTES and MERIT Working Groups that during the campaign (a) very long baseline radio interferometric and satellite and lunar laser ranging systems be used for co-located observations 
of high precision at the recommended sites, and (b) observations be made intensively for a limited period to detect any short-period variations in the derived Earthrotation parameters, and

urges that the appropriate resources and facilities be made available for these activities by the countries involved.

\section{AIG Resolution 2}

The International Association of Geodesy

considers that it is important that the new terrestrial reference frame to be derived fram high-precision observations during the MERIT Main Campaign should be extended and related to existing services as accurately and quickly as possible, and

urges that coordinated precise positioning observations be made during the campaign by satellite radio-tracking systems at the Very Long Baseline Interfercmetric, Lunar and Satellite Laser Ranging sites as well as at a larger number of well distributed sites around the world.

It recommends (a) that the coordinates of the stations of the International Latitude Service be determined in the Reference System (NWL 10F) adopted for the analys is of observations by the Doppler method of artificial satellite of TRANSIT or NOVA type, and (b) that special support be given to BIH acting as the coordinating center for the MERIT international campaign.

In a meeting devoted to regularized UT1: $B$. Guinot described the zonal tidal effects on the rotation angle of the Earth. Up to $+0.8 \mathrm{~ms}$ error may appear in UT1 because of failure to account for this ef fect. $\bar{D}$.D. MaCarthy then reported on the need for clarification of the use of UT1*. Comparison of U.S. Naval Observatory UT1 and UT1 derived from BIH UT1* shows that USNO UT1 is in better agreement with UT1 derived from the regularized UT1.

S. Yatskiv showed that regularized UT1 could be used practically in an ephemeris terrestrial system used as an reference system intermediate between the Conventional Inertial System and the Conventional Terrestrial System.

D.S. Robertson reported that he found no improvement when he campared VLBI results with UT1 derived fram UT1*.

A discussion then followed which concerned some of the details of a proposed resolution regarding the zonal tidal model and the need for more careful clarification. Action on the proposal was postponed to a later meeting.

\section{ADMINISTRATIVE SESS ION}

In the first business meeting of Carmission 31 , S. Iijima presented the President's Report. Members stood in silence to honor the memory of N. Matsunami and A. V. Shiryaev who passed away since the last meeting in Montreal. This was followed by a report delivered by $B$. Guinot on the Bureau International de l'Heure. He stated that there were no major changes in the formulation of TAI, and that clocks and frequency standards from N. America, Europe and N. Africa continued to be intercompared using Loran-C. The AlaOs algorith produces EAL fram which TAI is formed using primary cesium standards. Steps in frequency of $\pm 0.2 \times 10^{-13}$ are made with about two steering corrections per year. 
An annual effect in the difference between TAI and the primary standards was noted. The omplitude is about 1 microsecond and it is not clear what the cause is al though Guinot feels that it is probably due to camercial clocks. Work is now in process on a new version of ALCas.

The maximum error in TAI for averaging times greater than two months is $+1 \times 10^{-13}$. The BIH expects to cooperate in Project LASSO. Guinot noted that the relationship with the International Bureau of Weights and Measures provides the services of one physicist and remains very satisfactory.

W. Markowitz stated that his report on the meeting of the Consultative Camittee for the Definition of the Second (CODS) held in Paris in September 1980, had already been published and is available in the IAU Information Bulletin of January 1981.

J. Pilkington delivered the report of H.M. Smith on the International Radio Consultative Camittee (OCIR) Study Group 7. He mentioned that OCIR reports have not been so widely known, but contain much useful information for investigators in the field. The quality of the work of BIH was also recognized by the OCIR and this was reported to the Cammission.

S. Aoki concluded the session by presenting a discussion on the aspects of legal time. This included a compilation of the reports fram Camission members regarding existing legislation in their respective countries concerned with time. His report will be published elsewhere, e.g., in the Time and Latitude Bulletins of the Tokyo Astronamical Observatory.

\section{$\underline{20 \text { August } 1982}$}

A joint meeting with Cammissions $4,7,8$ and 19 was held for the discussion of resolutions related to the adoption of new astronamical coordinate systems, constants and theories in 1984. (See the report of Cammission 4.)

In an extra meeting of Commissions 19 and 31 regarding Regularized UT1 which followed, I.I. Mueller briefly reviewed the discussion of the previous day. B. Guinot presented a new resolution regarding the use of a zonal tide model. The following resolution was passed with 21 in favor and 1 abstention:

International Astronomical Union Commissions 19 and 31

considering the need to clarify the role of the zonal tides in the processing and publication of Universal Time

recommend (1) that, when the effect of the short period zonal tides, i.e., with (periods less than 35 days), is camputed for the above purposes, the attached expression based on Yoder, Williams and Parkes study (J. Geophys Res., 86, 881 1981) be used, and (2) that the letter $R$ be added to the notation of the relevant quantities to indicate that the correction for the short period zonal terms has been made (for instance UT1R).

l'Union Astronomique Internationale Camissions 19 et 31 Considerant le besoin de représenter clairement le role des marées zonales dans le calcul et la publication de temps universel, recammandent que lorsque l'effet des marées zonales à court terme (période inferieure à 35 jours) est calculé dans les buts précités, on utilise l'expression ci-jointe, tirée des travaux de Yoder, Williams et Parke (J. Geophys Res., 86, 881 (1981), et que le suffixe R soit ajouté à la notation des quantities concernées pour signifier que la correction des effets à court terme a été effectuée (exemple: UT1R). 
TERIS DUE TO ZONAL TIDES, WITH PERIODS UP TO 35 DAYS

Based on Yoder, Williams and Parke: 1981, J. Geophys Res., Vol 86, 881 with $\kappa / C=0.94$

UT1R, $\Delta R, \omega R$ represents the regularized forms of UT1, of the duration of the day $\Delta$, and of the angular velocity of the Earth, $\omega$.

The units are $10^{-4} \mathrm{~s}$ for UT, $10^{-5} \mathrm{~s}$ for $\Delta$, and $10^{-14} \mathrm{rad} / \mathrm{s}$ for $\omega$.

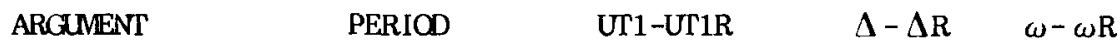

\begin{tabular}{|c|c|c|c|c|c|c|c|c|c|}
\hline $\mathbf{N}$ & 1 & $1^{\prime}$ & $\mathbf{F}$ & $\mathrm{D}$ & $\Omega$ & Days & $\begin{array}{l}\text { Coefficient of } \\
\text { Sin (Argument) }\end{array}$ & \multicolumn{2}{|c|}{$\begin{array}{l}\text { Coeff i cient } \\
\text { Cos (Argume }\end{array}$} \\
\hline 1 & 1 & 0 & 2 & 2 & 2 & 5.64 & -0.02 & 0.3 & -0.2 \\
\hline 2 & 2 & 0 & 2 & 0 & 1 & 6.85 & -0.04 & 0.4 & -0.3 \\
\hline 3 & 2 & 0 & 2 & 0 & 2 & 6.86 & -0.10 & 0.9 & -0.8 \\
\hline 4 & 0 & 0 & 2 & 2 & 1 & 7.09 & -0.05 & 0.4 & -0.4 \\
\hline 5 & 0 & 0 & 2 & 2 & 2 & 7.10 & -0.12 & 1.1 & -0.9 \\
\hline 6 & 1 & 0 & 2 & 0 & 0 & 9.11 & -0.04 & 0.3 & -0.2 \\
\hline 7 & 1 & 0 & 2 & 0 & 1 & 9.12 & -0.41 & 2.8 & -2.4 \\
\hline 8 & 1 & 0 & 2 & 0 & 2 & 9.13 & -0.99 & 6.8 & -5.8 \\
\hline 9 & 3 & 0 & 0 & 0 & 0 & 9.18 & -0.02 & 0.1 & -0.1 \\
\hline 10 & -1 & 0 & 2 & 2 & 1 & 9.54 & -0.08 & 0.5 & -0.5 \\
\hline 11 & -1 & 0 & 2 & 2 & 2 & 9.56 & -0.20 & 1.3 & -1.1 \\
\hline 12 & 1 & 0 & 0 & 2 & 0 & 9.61 & -0.08 & 0.5 & -0.4 \\
\hline 13 & 2 & 0 & 2 & -2 & 2 & 12.81 & 0.02 & -0.1 & 0.1 \\
\hline 14 & 0 & 1 & 2 & 0 & 2 & 13.17 & 0.03 & -0.1 & 0.1 \\
\hline 15 & 0 & 0 & 2 & 0 & 0 & 13.61 & -0.30 & 1.4 & -1.2 \\
\hline 16 & 0 & 0 & 2 & 0 & 1 & 13.63 & -3.21 & 14.8 & -12.5 \\
\hline 17 & 0 & 0 & 2 & 0 & 2 & 13.66 & -7.76 & 35.7 & -30.1 \\
\hline 18 & 2 & 0 & 0 & 0 & -1 & 13.75 & 0.02 & -0.1 & 0.1 \\
\hline 19 & 2 & 0 & 0 & 0 & 0 & 13.78 & -0.34 & 1.5 & -1.3 \\
\hline 20 & 2 & 0 & 0 & 0 & 1 & 13.81 & 0.02 & -0.1 & 0.1 \\
\hline 21 & 0 & -1 & 2 & 0 & 2 & 14.19 & -0.02 & 0.1 & -0.1 \\
\hline 22 & 0 & 0 & 0 & 2 & -1 & 14.73 & 0.05 & -0.2 & 0.2 \\
\hline 23 & 0 & 0 & 0 & 2 & 0 & 14.77 & -0.73 & 3.1 & -2.0 \\
\hline 24 & 0 & 0 & 0 & 2 & 1 & 14.80 & -0.05 & 0.2 & -0.2 \\
\hline 25 & 0 & -1 & 0 & 2 & 0 & 15.39 & -0.05 & 0.2 & -0.2 \\
\hline 26 & 1 & 0 & 2 & -2 & 1 & 23.86 & 0.05 & -0.1 & 0.1 \\
\hline 27 & 1 & 0 & 2 & -2 & 2 & 23.94 & 0.10 & -0.3 & 0.2 \\
\hline 28 & 1 & 1 & 0 & 0 & 0 & 25.62 & 0.04 & -0.1 & 0.1 \\
\hline 29 & -1 & 0 & 2 & 0 & 0 & 26.88 & 0.05 & -0.1 & 0.1 \\
\hline 30 & -1 & 0 & 2 & 0 & 1 & 26.98 & 0.18 & -0.4 & 0.3 \\
\hline 31 & -1 & 0 & 2 & 0 & 2 & 27.09 & 0.44 & -1.0 & 0.9 \\
\hline 32 & 1 & 0 & 0 & 0 & -1 & 27.44 & 0.53 & -1.2 & 1.0 \\
\hline 33 & 1 & 0 & 0 & 0 & 0 & 27.56 & -8.26 & 18.8 & -15.9 \\
\hline 34 & 1 & 0 & 0 & 0 & 1 & 27.67 & 0.54 & -1.2 & 1.0 \\
\hline 35 & 0 & 0 & 0 & 1 & 0 & 29.53 & 0.05 & -0.1 & 0.1 \\
\hline 36 & 1 & -1 & 0 & 0 & 0 & 29.80 & -0.06 & 0.1 & -0.1 \\
\hline 37 & -1 & 0 & 0 & 2 & -1 & 31.66 & 0.12 & -0.2 & 0.2 \\
\hline 38 & -1 & 0 & 0 & 2 & 0 & 31.81 & -1.82 & 3.6 & -3.0 \\
\hline 39 & -1 & 0 & 0 & 2 & 1 & 31.96 & 0.13 & -0.3 & 0.2 \\
\hline 40 & 1 & 0 & -2 & 2 & -1 & 32.61 & 0.02 & -0.0 & 0.0 \\
\hline 41 & -1 & -1 & 0 & 2 & 0 & 34.85 & -0.09 & 0.2 & -0.1 \\
\hline
\end{tabular}

$1=134.96+13 \% 064993(\mathrm{MJD}-51544.5)$ Mean Anamaly of the Moon

$1^{\prime}=357.53+0.985600($ MJD-51544.5) Mean Anomaly of the Sun 


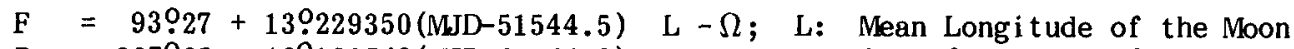

$\mathrm{D}=297.85+12.190749(\mathrm{MJD}-51544.5)$ Mean Elongation of the Moon from

$\Omega=125.04-0.052954(\mathrm{MJD}-51544.5)$ Mean Long $\mathrm{itude}$ of the Ascending Node of the Moon

A short discussion followed concerning a possible sign change in the pole coordinates to reflect the adoption of a positive East longitude convention. No act $i$ on was taken.

Joint meetings were also later held with Cammissions 4 and 19 which were concerned with astronamical constants and ephemerides. (See report of Cammission 4.)

\section{$\underline{23 \text { August } 1982}$}

In a scientific session H.F. Fliegel reported on Time Transfer using the Global Positioning System (GPS). This system of navigational satellites is designated by the United States Department of Defense as the successor to the TRANSIT satellite system. GPS was originally proposed as a constellation of 24 satellites, which would have provided a cont inuous worldwide navigational capability. It is now to be implemented as an 18-satellite system, of which 4 satellites will almost always be more than $5^{\circ}$ above the horizon. Four of the proposed 18 satellites are now operating, and are designated Navstars $3,4,5$ and 6. They transmit two codes: a $P$ (precision) code, which is intended to be available in the final system only by authorized users; and a C/A (clear access) code, which is, and which is expected to remain, available to all users.

Fliegel reviewed the time transfer experiments made since the last IAU General Assembly using the C/A code of the GPS system, especially between the U.S. National Bureau of Standards and the U.S. Naval Observatory.

B.E.H. Serene presented a paper entitled "The LASSO Experiment Readiness Prior to SIRIO-2 Launch". The launch is scheduled for 10 September 1982. In the paper, Serene described the laser pulse system. It is expected that $+1 \mathrm{~ns}$ accuracy can be achieved in time transfers among Austria, France, East and West Germany, I taly, Netherlands, Spa in, USA, and ESA. The first activity will be operated fram CERGA in order to use the laser ranging facilities there. This will consist of getting the satellite into position over longitude $25^{\circ} \mathrm{W}$, allowing the crystal to warm up and conducting experiments with signal detections and threshold sensitivity.

S. Leschiutta then reported on LASSO follow-on activities. Scient if ic needs which will be addressed include navigation, precise time transfer, VLBI, and the metrological system.

The error sources in LASSO are expected to be due to the width and shape of the pulse, the stability of the amplitude, and optical and electrical delays.

Other effects on the the satellite orbit such as attitude control, and possible weather dependence may be a source of concern. All of this activity will require coordination of synchronous activity of many groups. The advantages include the facts that there is no ionosphere effect and no problems with military usage. Accuracy of the time transfer is hoped to be about $+1 \mathrm{~ns}$. Further activity may be concerned with the development of microwave and optical systems. Leschiutta then went on to describe the planning of a second generation SIRIO, the POP satellite (POPSAT) for positioning, polar motion, Earth rotation and the Precise Range and Range Rate Satellite (PRARE). 
S. Starker presented a paper entitled "The Use of Spread Spectrum Techniques for Time Transfer". He pointed out that pulse techniques require a strong pulse and a high signal-to-noise ratio, while spread spectrum techniques require relatively low power and low signal-to-noise. He described an experiment which used the OTS-2 satellite which, despite a problem with multipath, gave clock difference accuracy of $\pm 20 \mathrm{~ns}$.

J.A. Galt reported on Satellite Time Comparisons by OW Techniques. He discussed the use of the Symphonie Satellite in which precisions of $1 \times 10^{-14}$ were obtained routinely between Canada and PTB. This method is no longer used since satellite ground stations are not available. Galt also mentioned the ANIK A1 satellite which is experimental but has achieved \pm 1 ns precisions over very small distance.

C.O. Alley presented a paper entitled "Sub-ns Time Camparisons Using 100 ps Laser Pulses" in which he described a joint U.S. Naval Observatory - University of Maryland experiment in time transfer. A standard deviation of 100 comparisons of $\pm 200-400$ ps was achieved and the accuracy is estimated to be 1-2 ns over a $26-\mathrm{km}$ separation. Alley also described the Shuttle Time and Frequency Transfer (STIFT) experiment which has a goal of \pm 1 ns in time and $\pm 1 \times 10^{-15}$ in frequency transfer.

A paper entitled "Time Transfer by VLBI" was presented by J. Fanselow in which he listed the delays encountered in VLBI time transfer. These include geometric, media (ionospheric, tropospheric,....) antenna, instrument, as well as the clock difference. The Deep Space Network of the Jet Propulsion Laboratory has a water vapor radiameter capable of $+3 \mathrm{~ns}$. Within two years it is expected that this will be reduced to a precision better than \pm 1 ns and that time transfer accurate to hundreds of picoseconds may be possible.

Ya. S. Yatskiv presented a paper entitled "Pulsar Time Scale" by V.G. Il'in, Ya. P. Ilyasov, A.D. Kuzmin, S.B. Pushkin, G.N. Palij, T.Y. Shabanova, Yu. P. Shitov. The authors propose to use pulsars for possible time transfer at an accuracy level of about 200 microseconds. The pulsars are PSR $0834+06$ with a period of 1.73 and a stability of $6.8 \times 10^{-15}$; PSR $0950+08$ with a period of 0.25 ; and PSR $1919+21$ with a period of 1933 . Discussion of the paper pointed out the existence of randam noise in the rates of pulsar timings and the requirement for large instruments and possible secular rates in pulsar timing.

S.-h. Ye presented a paper entitled "Time Camparison Equipment via Laser Pulses" by F.-m. Yang, Q.-x. Zhuang, J.-y. Su, D.-t. Tan, Z.-y. Li, and J.-x. Cai. She reported on time transfer experiments over a $25.2 \mathrm{~km}$ distance which achieved an external precision of $\pm 1.5 \mathrm{~ns}$. This was done using Rubidium clocks and it is expected that results would be improved with improved clocks. She feels that an internal precision of $\pm 0.5 \mathrm{~ns}$ is possible and is eager to participate in experiments with other laboratories.

In a joint session with Cammission 4 the following resolution on UT1 as the argument of almanacs was discussed and passed with 27 in favor and one abstention:

International Astronomical Uni on Cammissions 4 and 31

Noting that the present method of keeping UTC within 0.9 of UT1 by means of leap seconds both provides the second of SI and meets the needs for safe celestial navigation,

Recormend that the Air and Nautical Almanacs continue to be published with UT1 as the argument. 
Explanatory Note This resolution concerns only the cont inued use of UT1 as the argiment of navigational almanacs. It does not deal with the use of less precisely defined terms, such as GMT and UT, which are used as tabular headings in the sense of UT1. Recommendations concerning the ir use were made in Resolut i on Number 1, adopted by Commissions 4 and 31 in 1976 at Grenoble.

l'Union Astronomique Internationale Cammissions 4 et 31 notant que la methode actuelle qui maintient le UTC a moins de $0.9 \mathrm{~s}$ du UT1 au moyen de secondes intercalaires procure la seconde du $\mathrm{S} 1$ et remplit les besoins pour une navigation astronamique sure, recormandent que les éphémérides aeronautiques et nautiques, continuent à étre publi ées en utilisant UT1 comme argument. Note d'explication Cette résolution ne se rapporte qu'á la poursuite de l'usage de UT1 conme argument des éphémérides de navigation. Elle ne traite pas de l'usage de termes moins précisenent definis, tels que GMT, UT qui sont utilisés dans les tables avec les sens de UT1. Des recommandations sour l'emploi de ces termes apparaissent dans la Résolution no 1, adoptée par les cormissions 4 et 31 , en 1976 , à Grenoble.

A resolution recognizing the CODS Declaration on TAI was discussed and tabled.

Following those items $S$. Aoki presented a paper entitled "Operational Procedure of TAI". He discussed precise definitions of the geocentric frame and the geoid which are key concepts in the relationships between TAI, TDT and TDB. A proper reference frame of a non-rotating massless particle, whose trajectory coincides with the geocenter, is introduced as a representative geocentric frame. By this frame, TDT is defined through a theory of dynamies from TDB without considering any geophysical effect such as the geopotential, the Earth rotation, and the polar motion.

On the other hand, an atomic time can be connected to TDT by taking into account all geophysical effects within this frame. Fram our present knowledge of geophysical phenomena, it is possible to identify TAI with TDT within the accuracy of the order of $10^{-16}$. He noted, however, that TAI is in principle a "coordinated" time scale defined by certain operational procedure which may be changed according to our knowledge about the Earth.

W. Markowitz then described the relationship between Ephemer is Time, TDT and the present extrapolation of Ephemeris Time obtained by using TAI. He reported that it is not yet possible to detect a linear drift in the number of periods of Cesium-133 radiation in one Ephemer is Time second.

A paper prepared by the Hydrographic Department of Japan showing lunar occultation observations of ET-TAI was presented by A. Yamazaki. On 1 January 1981, the Hydrographic Department of Japan (JHD) took over the services of the international center for the receipt and processing of timings of Lunar occultations from H.M. Nautical Almanac Office.

Since then JHD:

1. set up "International Lunar Occultation Center" in the Astronamical Division;

2. accepted 17,866 timing data in total $(11,362$ in 1981 and 6,504 in 1982 ) as of July 31,1982 ; 
3. checked and corrected these data and converted same of them into machine-readable forms;

4. computed the predictions of total and grazing occultations and sent to same institutions and groups;

5. edited a pamphlet "Guide to Lunar Occultation Observations" and distributed to all the observers.

The processing of the data is now in progress and results will be obtained soon.

\section{ADMINISTRATIVE SESSION}

The officers and Camission representatives were selected as follows:

President: G. Hermleb

Vice President: D.D. McCar thy

Organizing Committee: N.S. Blinov, C.C. Costain, H.F. Fliegel, B. Guinot, S. I i jima, Y. Mi ao, A. Orte, P. Paquet, E. Proverbio, J. Pilkington

Representative to CODS: W. Markowitz (J. Benavente, al ternate)

Representative to CCIR Study Group 7: H.M. Smith (J. Pilkington, alternate)

BIH Directing Board: P. Paquet, S. I i jima

New Commission Members: D.W. Allan, S. Aoki, K. Dorenwendt, M.-k. Fuj imoto, C. Kakuta, A.M. Lozinskij, D. Matsakis, D.S. Robertson, J.McA. Steele.

Consultants: C.O. Alley, J.A. Barnes, P. Gi acamo, R. Kaarls, Y. Koga, R. Lake, S. Leschiut ta, B.S. Mathur, P. Mour ilhe Silva, J. Rutman, Y. Saburi, S. Starker, J. Terrien.

The cormission expressed its thanks to H.M. Smith for his past service on the BIH Directing Board. 
COMMISSION 33: STRUCTURE AND DYNAMICS OF THE GALACTIC SYSTEM (STRUCTURE ET DYNAMIQUE DU SYSTEME GALACTIQUE)

Report of Meetings on 18,20,21,23,24 and 25 August 1982

ACTING PRESIDENT: R. Wielen SECRETARY: W.B. Burton

18 August 1982

Business Meeting of Commission 33

Due to illness, the President of the Commission, G.G. Kuzmin, was unable to attend the General Assembly. Therefore, the Vice-President, R. Wielen, was appointed as Acting President.

The Commission unanimously approved the election of the new President, R. Wielen, and Vice-President, W.B. Burton. The Commission unanimously accepted the list of names proposed for the new Organizing Committee (continuing until 1985: W. Iwanowska, E.K. Kharadze, G.G. Kuzmin as outgoing president, D. LyndenBe11; for the period 1982-1988: L. Blitz, G. Lynga, M. Mayor, M. Miyamoto). The list of IAU members who wish to join Commission 33 was read and adopted:

G. Asteriadis, L. Balazs, B. Baud, L. Blitz, H.V. Cane, J.Y. Caswell, N.F. Comins, J.K. Denoyelle, G.F. Gilmore, K. Hamajima, S. Inagaki, M. Iye, R.N. Manchester, T. Papayannopoulos, B.J. Robinson, H. Schwerdtfeger, J.A. Sellwood, A. Strobe1, C. Terzides, K.0. Thielheim, W. Tobin, S.S.O. Wrandemark, Y. Yoshii.

Recent and future meetings were discussed. W.B. Burton reported on the IAU Symposium No. 100 "Internal Kinematics and Dynamics of Galaxies" in Besancon and on the Leiden Southern Workshop; L. Blitz summarized the Vancouver Workshop on "The Milky Way. Structure, Kinematics and Dynamics" (Editor of the proceedings: W. L.H. Shuter; to be published in the Reidel Astrophysics and Space Science Library). R. Wielen reported that Commission 33 is sponsoring an IAU Symposium on "The Milky Way as a Galaxy" in Groningen, June 1983, and is co-sponsoring an IAU Colloquium on "Nearby Stars and the Stellar Luminosity Function" in Middletown, June 1983. Commission 33 also supported a proposal for a Colloquium on "Statistical Methods in Astronomy" in Strasbourg. R. Wielen described the program for the scientific sessions of Commission 33 at the Patras General Assembly.

After a lengthy discussion, Comnission 33 decided to establish a Working Group on Galactic Constants. The purpose of the working group is (1) to consider whether any changes in the system of galactic constants $R_{o}, A, B, \theta_{o}$ should be recommended and (2) to produce a critical review about the available data on these galactic constants and on other quantities (e.g. the solar motion). F. Kerr will be the chairman of the working group, D. Lynden-Bell the vice-chairman.

\section{Observing Program for the Astrometry Satellite HIPPARCOS}

Chairman: R. Wielen. The following papers were presented:

R. Wielen: Introduction: Possible contributions of IAU Commission 33 to the HIPPARCOS observing program

C.A. Murray: Scientific organisation of the HIPPARCOS project 
C. Turon: Compilation of the Input Catalogue for HIPPARCOS

A.R. Upgren: Trigonometric parallaxes from HIPPARCOS

R. Wielen: Proper motions from HIPPARCOS

J.P. Garrigue, J. Guibert, M.O. Mennessier, N.Q. Rieu: HIPPARCOS and the variable stars

E. Hфg: TYCHO, an astrometric and photometric survey

\section{August 1982}

Joint Meeting of Commissions 33 and 28. Chairman: D. Lynden-Be11. The following papers were presented:

J.P. Ostriker: Review of dark haloes

H. van Woerden: Masses of galaxies from $21 \mathrm{~cm}$ curves and binaries

A.J. Kalnajs: Interpretation of velocity curves

J.E. Baldwin: Velocity curve of M31

A. Pedlar: Mass-to-light ratios in small groups of galaxies

J. Hunter: Velocity curve for a galaxy with satellites

E.E. Salpeter: Evidence on dark haloes and $M / L$ in clusters of galaxies

D.N. Schramm: Cosmological constraints on the constitution of dark haloes

R.B. Tully: Interpretation of Virgo Cluster dynamics

\section{August 1982}

Selection of Objects for Observations by HIPPARCOS and Space Telescope for the Establishment of an Extragalactic Reference Frame

Joint Meeting of Commissions 24,7,8,33, 40 and 44. Chairman: A.N. Argue. See report of Commission 24.

\section{August 1982}

\section{The Large-Scale Distribution of Gas in the Galaxy}

Joint Meeting of Commissions 33 and 34. Chairman: W.B. Burton. The following papers were presented:

L. Blitz: Mass and distribution of hydrogen, especially in the outer parts of the Galaxy

I. Kazès: Distribution and physical state of HI seen in absorption

$D . B$. Sanders: Distribution of $C O$ in our Galaxy and in nearby spirals

B. Robinson: Distribution of $\mathrm{CO}$ from southern-hemisphere surveys

R.J. Cohen: Distribution of molecules other than $\mathrm{CO}$ and $\mathrm{H}_{2} \mathrm{O}$

W.L.H. Shuter: The al1-plane variation of sine with $v$

B.E. Turner: Mapping of the $1720 \mathrm{MHz}$ OH line

H. Bloemen: $\gamma$-rays and the galactic gas distribution

C. Heiles: Supershells as a characteristic of the galactic gas morphology

R. Giovanelli: High-velocity clouds

I. Mirabel: High-velocity clouds near the direction of the galactic center

A. Winnberg: The total mass in the inner Galaxy

I. Kazès: HI absorption lines from molecular clouds 
24 August 1982

Recent Results on Galactic Radio Sources and mm Waves

Joint Meeting of Commissions 40, 33 and 34. Chairman: G. Swarup. See report of Commission 40.

\section{August 1982 \\ Dynamics}

Chairman: R. Wielen. The following papers were presented:

G. Contopoulos: Ordered and ergodic motions in galaxies

E. Athanassoula: Periodic orbits and ergodicity in barred galaxy models

S. Ninkovic: Contribution to the study of the structure and dynamics of the galactic halo

A full account of the Joint Discussion II on the "Evolution of old stellar populations", co-sponsored by Commission 33, is given in the "Highlights of Astronomy". 
COMMISSION 34: INTERSTELILAR MATTER

(MATIERE INTERSTELLAIRE)

Report of Meetings, $20-25$ August 1982

PRESIDENT: V. Radhakrishnan

VICE-PRESIDENT: M. Peimbert

SECRETARY: D.C.V. Mallik

Business session, 20 August

The President and members honoured the memory of $\mathrm{K}$. Serkowski, a member of the Commission, who died on October 7, 1982, and of B.M. Tinsley who died on 23 March 1981.

The President then gave a short report on the Commission's activities during the triennium. The Commission 34 Report for 1982 had been prepared in consultation with the members of the Organising Committee. The President thanked all the contributors for their help, and mentioned that the Report had been commented upon very favourably by the General Secretary of the Union.

\section{Membership}

According to the list passed on to the President by the IAU Secretariat the membership of the Commission stood at 478, registering a healthy growth since the last General Assembly. Four more names were added during the Business Session bringing the total to 482 . The President noted that although this made the Commission one of the largest in the Union the number was not unmanageable in any sense and no measure was needed to curb it.

\section{Organising Committee}

Next, the slate of officers and Organising Committee for the next triennium was approved with one change necessitated by B.D. Donn's preference to step down from the 0.C. Following a proposal by M. Peimbert, his place was taken by J.S. Mathis. The Committee as proposed for 1982-85 now consists of M. Peimbert President; J. Lequeux, Vice-President; S. D'Odorico; B.G. Elmegreen; E.B. Kostyakova; J.S. Mathis; U. Mebold; V. Radhakrishnan; P.A. Shaver; Y. Terzian, P.G. Wannier.

\section{Change of Name of Commission}

The Presldent proposed that the name of Commission 34 should be changed to simply "Interstellar Matter" from "Interstellar Matter and Planetary Nebulae" since planetary nebulae are only one of various types of objects the Commission deals with and as such there was no need for their mention in the Commision title. In the discussion that followed, L.H. Aller, perhaps the oldest member of the Commission present in the Business Meeting, noted that the proposed new name of the Commission was really not new since the Commission was called by this name in the early forties when he became a member. Peimbert noted that planetary nebulae were different in the sense that their study includes the study of the central.stars also. He felt that a Working Group on Planetary Nebulae may be formed to coordinate the work in this special area. By agreement the decision on the formation of the WG was postponed to the next IAU General Assembly. The 
President allayed the fears of some of the members by stating that the change in the name did not imply any change in the activities of the Comission, and that planetary nebulae would continue to be one of the important areas of interest of the Commission. The members agreed with the proposed change of name.

\section{Symposia/Colloquia}

The Vice-President then listed the following meetings under consideration which may be of interest to members of the Commission:

1. "Milky Way as a Galaxy", Groningen, The Netherlands, May-June 1983.

2. "Mage1lanic Clouds", Tubingen, FRG, 1983.

3. "Nearby Interstellar Medium", U.S.A., 1983-84. The venue is yet to be decided.

4."Star Forming Regions", Tokyo, Japan, 1985.

5. "Planetary Nebulae", Mexico, D.F., Mexico or Santa Cruz, U.S.A., 1987.

\section{Other Matters}

The President then called on Helene Dickel, coordinator of the Working Group on Nomenclature, to give a report of their activity. Dickel mentioned the efforts of the WG towards evolving a standardised nomenclature in areas of interest to Commission 34, and reported in particular on the publication of two compendiums: (1) The First Dictionary of Nomenclature of Celestial objects by A. Fernandez, M.C. Lortet and F. Spite, and (2) A Compilation of Current Designation Practices in Astronomy by J. Mead. In view of the good work done by the Working Group $1 t$ was decided that the Group should continue. In response to requests for a Working Group on Calibration of HI Profiles, U. Mebold, a member of the OC, was named as the coordinator to form such a group:

\section{Scientific Sessions}

The President spoke briefly on the planning of the scientific sessions during the current General Assembly. The programme, he said, was decided after extensive correspondence with members of the $\mathrm{OC}$ and Presidents of other Commissions. Commission 34 is involved in Jolnt Discussion II on Evolution of old Stellar Populations in Galaxies and five Joint Meetings with various comissions. In addition, a session of contributed papers on Important Recent Results and a session on Planetary Nebulae have also been organised. The members expressed satisfaction at the general planning of these sessions. However, some members felt that a single session on contributed papers was highly inadequate and at least two such sessions should be planned in future. It was suggested that more papers could be accommodated by having poster sessions in addition to oral presentations, although opinion was divided on the suitability of such a session during an IAU General Assembly.

Brief reports on the scientific sessions are given below.

\section{JOINT COMMISSION MEETINGS}

\section{The Violent Interstellar Medium}

Commissions 34,40 , and 48

Chairman: R. McCray

20 August 1982

Following the brief introductory remarks by the Chairman, de Boer reviewed the data on the high velocity gas in the galactic disk and halo. Giving a 
brief historlcal perspective on the ploneering work in the optical region by Adams, Spitzer, Munch and others, he went on to describe in some detail the wealth of information obtained in UV by Copernicus and IUE. This included the observations of high velocity motions in galactic H II regions. Although there Is reasonable coverage of the velocity data in many parts of the sky, a consistent theoretical model describing the motions of the gas both in the disk and the halo is yet to emerge. de Boer mentioned in this connection the theoretical work of Shapiro and Field and of Bregman on the Galactic Fountain. Very recently, observations by de Boer and Savage of the interstellar lines in the spectrum of Barnard 29 in M13 have suggested that the halo gas does not corotate with the disk as was believed earlier. The data suggest a decrease in the rotational velocity with increasing distance from the plane.

The high velocity motions and complex structure of the Carina region were discussed by Walborn who showed spectacular [S II] interference filter photographs of the region. The data on both the optical and UV interstellar lines of the region indicate high velocities in the range $-200 \mathrm{kms}^{-1}$ to $+150 \mathrm{kms}^{-1}$. The Carina region is also a source of soft $X$-rays as observed by the Einstein Observatory. According to Walborn, the sources of the kinetic energy in the region are derived from the energetic wind of the of and WR stars found in the region. The question as to whether the region contains an unrecognised SNR has not yet been settled.

Ever since the discovery of $\mathrm{H}_{2} \mathrm{O}$ maser sources in Orion and W49 more than a decade ago, observations in infrared and millimeter waves have clearly shown the existence of high velocity mass outflows in molecular clouds. More recent indications have come from the data on high rotational lines of co seen in the submillimeter reglons of the spectrum and from quadrupole lines in the vibrationrotation spectrum of $\mathrm{H}_{2}$. Reviewing the violent activity in dense molecular clouds, Genzel emphasized that the high excitation observed in molecular Iines in these reglons indicates the presence of hydromagnetic shocks. Further, a bipolar structure of the molecular outflows is seen to be an extremely common occurrence in these clouds as also in compact IR sources, very compact HII regions and in the neighbourhood of $\mathrm{T}$ Tauri stars and Herbig-Haro objects. Genzel described in detail the kinematic picture of the Kleinmann-Low region in Orion that has emerged from these observations. It seems unlikely that the outflows are radiatively driven in this region since the momentum in the flow is one to two orders of magnttude higher than the momentum in the radiation field. However, the radiative energy avallable is more than an order of magnitude higher than the kinetic energy in the outflows and a satisfactory mechanism is yet to be invoked for an efficient conversion of this energy.

The observational status of the soft X-ray background was discussed in the talks by Clark and Kraushaar. Clark reviewed the SAS 3 results and stressed that the observed $\mathrm{C}$-band intensity can be fitted by contributions from two components - a local one from a hot bubble in which we are immersed, and a remote or extended one due to the widely distributed galactic corona. The extended component is absorbed in transmission as is evident from an anticorrelation of the C-band intensity with the colum density of the neutral gas.. The effective absorption cross-section of the gas is, however, less than the photoelectric absorption, and this indicates a high degree of 'clumping' in the gas. Kraushaar's review included the higher energy $M$-band data. The existence of emission 1 ines in this band has conclusively proven the thermal nature of the X-rays. Although the $\mathrm{C}$-band and $\mathrm{B}$-band intensities anticorrelate with neutral hydrogen column density, the M-band Intensity, according to Kraushaar, does not show such an anticorrelation. Moreover, the origin of the $M$-band intensity is unknown since it requires substantial amounts of gas at $10^{6.4} \mathrm{~K}$ rather than at $10^{5.7} \mathrm{~K}$ as predicted by the McKee-Ostriker theory of a supernova-explosion-dominated ISM. 
Kraushaar conjectured that a part of the intensity might be extragalactic in orlgin, while the rest could be contributed by local $M$ dwarfs, although a fit to the data would then imply a local density of $M$ dwarfs much higher than the values quoted in stellar surveys.

Cash reviewed the observations of $\mathrm{X}$-ray supershells in the Cygnus, Orion/ Eridanus and Carina inner regions. These shells are invarlably associated with Giant Molecular Clouds and $O B$ associations with thetr outer boundaries usually delineated by optical $\mathrm{H} \alpha$ filaments. The absence of these shells in the Gum nebula and Carina outer regions impose stringent requirements on the thermal properties of the X-ray emitting gas. The optical supershells were reviewed by Meaburn who also showed beautiful $\mathrm{H \alpha}$ photographs of regions in the LMC and SMC containingthese structures. Meaburn emphasised that these are super-supershells in the sense that their physical dimensions are $\sim$ a kiloparsec, much larger than the $X$-ray supershells that Cash described. As a possible mechanism for producing these, Meaburn referred to the theory of stochastic self-propagating star-formation given by Gerola and Seiden. He concluded his review with a brief summary of recent speckle interferometric observations of R136 at the centre of 30 Doradus.

The concluding review of the session was given by Ostriker on the threephase model of the interstellar medium. He spoke briefly on the original version of the model by McKee and himself and described recent improvements of $1 t$ due to Cowie, McKee and himself. He discussed the theoretical and observational virtues of the model and also its problems.

The papers presented at the above session are expected to be published in the Highlights of Astronomy Vol. VI.

\section{Spectroscopic Investigation of Grains}

Commissions 15 and 34

Chairman: B.D. Donn

21 August 1982

The session opened with a review by Knacke on interstellar silicates. The existence of silicates in both interstellar and circumstellar dust seems to be well established through the identification of infrared bands at $9.8 \mu \mathrm{m}$ and $18 \mu \mathrm{m}$. The silicates are present in both diffuse and molecular clouds and their composition appears to be quite uniform since good fits to the observed spectra can be achieved by varying only the temperature and column density of dust in these sources. Polarization measurements show the silicate particles to be aligned and give quantitative limits on the band strengths. Knacke described the laboratory work on Brownlee particles whose spectra show remarkable similarities with the interstellar spectra, and condensation experiments that suggest silicates with spectra resembling the interstellar can condense from the gas. According to him, modelling shows that the visual and UV extinction and polarization data can be well fitted by silicates and graphite-like particles.

Kratschmer reported on the laboratory measurements on ice and ammonlated ice mixtures in the 4-18 $\mu \mathrm{m}$ range. Application of the data to the observed interstellar extinction characteristics suggests that for the BN object the $3.1 \mu \mathrm{m}$ feature together with its long wavelength wing may be described by spherical particles composed of $80 \% \mathrm{H}_{2} \mathrm{O}$ and $20 \% \mathrm{NH}_{3}$ with radil of $0.3-0.4 \mu \mathrm{m}$. Referring to the absence of the $12 . \mu \mathrm{m}$ absorption feature of ice in some IR sources, he said that this might be due to a deviation of the IR continuum beyond $10 \mu \mathrm{m}$ from a true blackbody, since the laboratory work did not indicate 
appreciable decrease in the strength of this feature due to the presence of admixtures.

Donn described the experimental research on grains carried out at Goddard, University of Alabama and York University. The program at Goddard is primarily a study of the condensation of refractory particles from the vapour and the determination of their various properties. A secondary phase of the program is a study of lce mixtures. These experiments have helped elucidate the nature of the particles in the interstellar medium that produce the $10 \mu \mathrm{m}$ and $20 \mu \mathrm{m}$ absorption bands and also the $3.4 \mathrm{\mu m}$ feature. He mentioned the work of Wdowiak on laboratory condensates. They showed absorptions at $4500 \AA, 6273 \AA, 6282 \AA$ and $5780 \AA$, and on warmup, emission features are seen in the range 5000-7500. Finally Donn referred to the laboratory work by Duley on MgO grains of size $<20 \mathrm{~nm}$ which suggests the presence of an absorption band at $220 \mathrm{~nm}$ similar to the interstellar UV absorption band. Mentioning the problems with graphite, Donn noted that further work on MgO would be desirable.

Pirronello described experiments on irradiation of ice mixtures with $1.5 \mathrm{MeV}$ helium ions to study the interaction of energetic particles with frozen gases. The last paper of the session was given by d'Hendecourt on expulsion of molecules from grains in dense clouds. Remarking on the existence of appreciable amounts of $\mathrm{CO}, \mathrm{H}_{2} \mathrm{O}$, and $\mathrm{NH}_{3}$ in these clouds, he suggested that grain-grain collisions at relative velocities above $40 \mathrm{~ms}^{-1}$ could produce molecular ejection from grains in clouds.

\section{Large-Scale Distribution of Gas}

Commissions 33 and 34

Chairman: W.B. Burton

23 August 1982

The mass and distribution of atomic hydrogen, distribution of co from northern and southern surveys, and distribution of molecules other than $\mathrm{CO}$ and $\mathrm{H}_{2}$ were discussed in reviews by B1itz, Sanders, Robinson and Cohen respectively. Heiles spoke on the HI supershells as a characteristic of the Galactic gas morphology. Kazes described work on HI observed in absorption. High-velocity clouds were discussed by Glovane111. A review on Gamma rays and the Galactic gas distribution was presented by Bloemen, and Young gave the final review on a comparison of the Galactic gas distribution with that in nearby spirals. A detailed report of this meeting will be found in the Commission 33 report in the Proceedings.

\section{Recent Results in Galactic Radio Astronomy}

Commissions 34 and 40

Chairman: V. Radhakrishnan

24 August 1982

Elghteen contributed papers were presented on topics which included Pulsars, Supernova Remnants, $H$ II regions and Spectral line observations of interstellar molecules. Among the more noteworthy results were the following.

Lyne reported on proper motion measurements on pulsars and an interesting correlation that has been found between their observed velocities and derived magnetic fields. M1lls presented observations of SNR in the LMC and SMC obtained with the recently commissioned Molonglo Synthesis Telescope. These observations 
clearly suggest that the majority of SNRs have not decelerated, contrary to the generally held view. Very beautiful observations of the Galactic Centre, obtained with the VLA, showing evidence of a spiral-1ike morphology for Sgr A - West and a shel1-structure for Sgr A - East were presented by Ekers. Jennison described the results obtained with the Mark I - Kent Low Frequency system which showed a jet-like structural anomaly in the SNR Cas A.

A complete 11st of all the papers will appear in the Comm. 40 reports in the Proceedings.

\section{The Interstellar Medium from Space}

Commissions 34 and 44

Chairman: W. Grewing

25 August 1982

The existence of a million-degree component of the interstellar gas is now well established. Garmire discussed the soft X-ray sky maps that have been obtained with the proportional counters aboard the HEAO 1 spacecraft, and argued that some of the extended features seen in these maps can be accounted for if the solar system is surrounded by million-degree gas extending out to a distance of about $100 \mathrm{pc}$. Within this volume must be a number of cool, evaporating clouds. At its edge one finds an expanding shock.

The same local region in space was then discussed by Bruhweiler who reviewed the results from recent UV experiments. It had been known already that the $H I$ column density is very low along a few lines of sight. A more systematic study has now shown that low $\mathrm{H}$ I column densities are found in the entire hemisphere between $1=90$ and $1=270$ degrees to distances of many tens of parsecs. Conversely, looking towards the galactic center, the line of sight seems to pass through a local cloud before penetrating again through a region with little $H$ I. Bruhweiler went on to discuss the implications that the very low H I densities have for the EUV radiation fleld in interstellar space.

The state of the interstellar gas at the outskirts of our galaxy was considered by Blades who reviewed the recent observations of the galactic halo, and haloes around external galaxies. In particular, the IUE observations of distant background targets provide clear evidence for gas well outside the plane of our galaxy. In addition to the predicted highly ionised species, one also finds evidence for neutral or only singly ionised constituents, indicating the presence of cool clouds. Indeed, in the very high resolution optical observations, many of the absorption components are seen to break up into several sub-components that are extremely narrow.

The contributions that infrared observations can make to extend our understanding of the interstellar medium were highlighted by Harwit, who chose the Orion complex as an example. The importance of various fine-structure transitions was stressed, and their observed strengths were compared to model predictions for $H$ II regions. Turning to very recent results, Harwit reported the detection of the diffuse [C II] $156 \mu \mathrm{m}$ emission from the galactic plane that was measured in a very beautiful experiment in which the moon was used as a reference source while it moved across the plane.

Ever since the interstellar reddening law was known from UV wavelengths through the optical to near and medium IR wavelengths, the question has been with us as to what materials cause the extinction. As Mathis pointed out in the concluding talk of the session, a vast amount of data has been complled over the 
past years, mostly from UV observations. He stressed that the features in the extinction curve are still a puzzle. While they can be attributed to a number of materials, all those materials would also produce additional features which are not observed. Mathis went on to discuss what can be concluded from the observed polarisation properties, from the peculiar extinction observed e.g., in dark clouds, and from the correlation with the atomic and molecular hydrogen densities. Some systematic effects are observed in the properties of circumstellar dust depending on the $0 / C$ ratio in the associated stars. Mathis concluded his review by stressing the need for further IR and FIR observations to be combined with observations in the UV.

COMMISSION 34 MEETINGS

\section{Important Recent Results}

Chairman: Y. Terzian

20 August 1982

The following contributed papers were presented:

Stellar Winds and Their Impact on the Interstellar Medium - Howard A. Smith. Multi-colour Polarimetric and Photometric Studies of the Rho Ophiuchi Dark Cloud - Frederick J. Vrba, G.V. Coyne, S. Tapia.

The Ratio of Deuterium to Hydrogen in Interstellar Space - claudine Laurent, Alfred V1dal-Madjar, Cecile Gry, Paul Bruston, Roger Ferlet, and Donald York.

Asymmetric Broad $\mathrm{HCO}^{+}$Line Wings in Cores of Molecular Clouds - Aa. Sandqvist, A. Wooten, R.B. Loren, P. Friberg, and A. Hjalmarson.

The D1ffuse Interstellar Lines at 5780 and $5797 \AA$ - D. McNally.

Infrared and Radio Observations of Broad-Winged CO Sources - Howard A. Smith, Harley A. Thronson, and Charles J. Lada.

Warm Neutral Halos Around Molecular Clouds - P.G. Wannier, and M.R. Morris. Detalled Models of Non-Spherical H II Regions - Robert Rubin.

Anomalous [SII] $6716 / \mathrm{H \alpha}$ Intensity Ratios in the Faint Galactic Background. - R.J. Reynolds.

Models of Molecular Clouds: Mult1-transition Studies of $\mathrm{CS}$ and $\mathrm{H}_{2} \mathrm{CO}$.

-Neal J. Evans II, Lee G. Mundy, Ronald L. Snell, and Paul F. GoIdsmith.

\section{Planetary Nebulae}

Chairman: M. Peimbert

21 August 1982

Terzian reviewed the observations of nebular shells. He noted that there had been major progress in observational techniques in the last few years. UV spectroscopy of the nebulae by IUE on the one hand, and high-resolution ( $\approx 0 ! 5)$ radiomaps obtained by the VLA on the other have enriched enormously our knowledge of the nebular shells. Recent observations have also revealed morphological detalls showing many of the nebulae to have more than one shell and sometimes 
halos. In her review of the central stars, Acker said that spectra of more than a hundred nuclei are now available. They show a wide range of characteristics w1th WR types perhaps dominating in number. Several of the visible nuclei are of late spectral types which, according to her, indicates the likely presence of hot companions. The binary nature of at least 16 planetary nuclei has been confirmed and 17 more are suspected to be binaries. She discussed the physical parameters of these stars and emphasised how greatly UV observations have helped in this respect.

Peach described briefly the important atomic processes in nebular shells and discussed the advances made in the calculations of atomic data in the last few years. The work of Mendoza on transition probabilities, electron excitation rates and photoionization cross-sections, of Nussbaumer and Storey on recombination processes, and of McCarroll and associates and Dalgarno and assoclates on charge exchange was mentioned in this connection. Aller spoke on the chemical abundances in planetaries and said that recent work had shown these to be sufficiently different from one nebular type to another so that an average chemical composition was no longer a meaningful characterisation Aller emphasised the advantages gained in recent times by the use of UV and infrared observations to obtain abundances and discussed the improved methods developed to do so.

Serrano described the planetary nebulae in a galactic context. Mentioning the difficulties in the determination of distances for the galactic planetaries, he quoted results from the surveys of such objects in Local Group galaxies to show that the total number in the Galaxy should be close to 10,000 . This, he said, favoured the calibration of distances by Cudworth over the one used by Cahn and Kaler. Serrano discussed the recent work on the determination of scale heights, space densities and birthrates of these nebulae in the solar neighbourhood and pointed out that the low value of the scale height obtained in general was a puzzle. He also discussed the significance of the carbon and nitrogen abundances of planetaries from the point of view of chemical evolution of galaxies.

The session ended with a contributed paper by Walborn on a suspected extended planetary nebula in LMC. 


\title{
23 August 1982
}

\section{BUSINESS MEETING}

At the start of the meeting members stood in silence for a few minutes in memory of B. M. Tinsley, who had died since the Montreal meeting.

The President reported that the vote which he had organised earlier in the year had produced the following list of nominations for membership of the Organising Commit tee :

\author{
PRESIDENT A. N. Cox (Los Alamos Scientific Laboratory, New Mexico) \\ VICE-PRESIDENT D. Sugimoto (University of Tokyo) \\ ORGANISING COMMITTEE P. Bodenheimer, C. Chiosi, D. J. Faulkner, A. Maeder, \\ J-L. Tassoul, R. J. Tayler, A. V. Tutukov, V. Weidemann, J-P. Zahn.
}

The composition of the Organising Committee was agreed. It was explained that the rule adopted at the Montreal meeting about the rotation of membership of the Organising Committee meant that D. J. Faulkner, R. J. Tayler, A. V. Tutukov and $\mathrm{J}-\mathrm{P}$. Zahn would leave the Organising Committee in three years' time. The ballot of members of the Commission had attracted not much short of a 50 per cent vote; this was quite a high vote as there are a number of senior members of the Commission who are no longer active in the field of stellar constitution.

The membership of the Commission was revised. Five new names had been added by the Executive Committee since the Montreal meeting; four of these were members from China (Nanjing) who were added when the arrangements for Chinese adherence to the IAU were settled and the fifth was an existing member who had intended to join at Montreal. The President had inspected the curricula vitae of all the new members who were now proposed by National Committees and he was satisfied that they were all active in the Commission's field. In addition some existing members of the IAU wished to join the Commission. The elsction of all of the new members was agreed. No member of the Commission, other than B. M. Tinsley, had been lost by death or resignation in the past three years. After the election of the new members, the Comission membership is 247.

The President announced that the Draft Report of the Commission had once again consisted of short reviews on specific topics with contributions by $V$. Castellani, J. P. Cox, A. Maeder, J. J. Monaghan, A. V. Tutukov and J. C. Wheeler. The small number of free offprints which he had received had been distributed to members of the outgoing and incoming Organising Committees and to the authors of contributions.

There was a discussion about the number of meetings of the Commission at Patras with some members feeling that there should have been more meetings. The President explained that there were two reasons why he had not tried to organise further meet1ngs. The first was that Commission 35 was involved in three Joint Discussions 
which were occupying three full days. The second was that he had received no suggestions for topics for Commission meetings following his circular letter earlier in the year. Dr Cox said that he would welcome the views of commission members on the number of meetings to be held at the next General Assembly and on topics for the meetings.

\section{Scientific Meetings}

MIXING IN STELLAR INTERIORS 20 AUGUST 1982

This meeting was organised by $A$. Maeder and had the following programme:

J-P. Zahn Mixing processes induced by stellar rotation

A. Renzini Surface chemical composition and mixing

A. Maeder Cluster observations and models with mixing

A fourth paper by G. Shaviv could not be given because he was unable to be at Patras but a short communication by $I$. W. Roxburgh on a new instability in stellar interiors was added to the programme.

Zahn said that mixing in stellar interiors could be produced elther by advection by large scale circulation or by instability leading to turbulence. The main cause of such instability was differential rotation, which could arise even in a star which was initialiy uniformly rotating as a result of contraction or expansion, of mass or angular momentum loss or of meridional circulation. He discussed four types of instability; shear flow, dynamical (Rayleigh), baroclinic and double diffusive. In particular in discussing baroclinic instability he stressed that boundary conditions are very important so that local stability criteria, which are relatively easy to obtain, are not very useful. Roxburgh also discussed baroclinic instability in the case of the Sun. He said that, if diffusion was present, there could be an overstability which might occur even if the well known Goldreich. Schubert, Fricke instability were absent. He suggested that the present solar angular velocity profile might be such as to give marginal instability, with the solar centre rotating much more rapidly than the surface, and that this might ease or solve the solar neutrino problem.

Renzini discussed the comparison between surface element abundances in red giants and the predictions of stellar evolution theory, in an attempt to decide whether mixing processes other than convection are needed. He showed that there is no compelling evidence for additional mixing in the first dredge-up stage in giants with masses < $3 M_{0}$. There are problems in Cepheids and GF supergiants, but these cannot simply be solved with an additional mixing process as the observations do not seem to conserve the total number of CNO nuclei which should not be changed in $H$ burning; some abundances are probably in error. There is apparently a need for a third dredge-up in low mass stars but this is not predicted by standard models.

Maeder explained that along the main sequence from $1 M_{0}$ to $60 M_{0}$ the observed main sequence band is wider than expected from standard theory. The effect increases with mass and it is not due to variation in chemical composition or to atmospheric effects. He discussed several detailed comparisons of theory and observation. In NGC 752 no hook is visible in the evolutionary tracks and in 34 clusters in the age range from the Hyades to the Pleiades there are ten times more stars about the main sequence band than expected. He discussed diffusion due to overshooting from convection zones and argued that this was necessary to explain the observations. 
Following the Business Meeting a Scientific Meeting was devoted to any topics of interest to members of the commission with the following programme:

K. A. van Riper
J. J. Cowan
A. N. Cox
Q-Y. Qu
C. M. Sharp
J. L. and M. Tassoul
G. Ruben
D. Sugimoto

K. A. van Riper

J. J. Cowan

J. L. and M. Tassoul

D. Sugimoto
Stellar collapse

r-process production in supernovae

Theoretical studies of Mira variables

Abnormal neutron stars and quark abnormal neutron stars Molecular opacities

Meridional circulation in early type stars

Meridional mixing in supernovae

Mass loss

van Riper discussed the pre-supernovae collapse of a massive star. The calculations of different groups disagree about whether or not an explosion occurs, which is not surprising as the gravitational energy released in a neutron star collapse is ten times the observed energy of a supernova. He discussed the role of nuclear disintegration, neutronization and neutrino trapping and explained that a core bounce should occur when it is slightly above nuclear density. Whether or not the envelope is ejected depends critically on the entropy distribution determined by earlier stellar evolution and on whether or not there is a phase transition just below nuclear density.

Cowan explained that although it is generally accepted that the r-process occurs in supernovae, its precise site is uncertain. There is a possibility that it can occur in the helium zone which is shocked as a result of the explosion. Provided that there is enough ${ }^{13} \mathrm{C}$ in the helium zone, neutrons provided by $(\alpha, n)$ reactions can produce the first two r-process peaks; the failure to produce the third peak may be due to errors in $\beta$-decay rates. An essential feature is that this is a non-equilibrium r-process. Provided a relatively small fraction of the helium zone has the correct conditions for the r-process, observed abundances can be explained.

Cox discussed some problems in the interpretation of the properties of Mira variables in 47 Tuc, in particular whether they pulsate in the fundamental mode or in the first overtone and suggested that a good treatment of time-dependent convection would be needed to solve the problem. Qu described an abnormal state of nuclear matter which could arise above nuclear density as a result of coupling between nucleons and mesons. In such circumstances the effective mass of the nucleon becomes very small and abnormal neutron stars may be possible with higher maximum masses than those of normal neutron stars. Both abnormal neutrons and abnormal protons can be important and models are also possible with a quark interior and an abnormal neutron exterior. He suggested that the change of state from a normal to an abnormal neutron star could produce a supernova explosion.

Sharp described his calculations of molecular opacities which might be relevant in the atmospheres of late type stars. He had considered a solar composition and had included 22 elements and 60 isotopes with 36 diatomic molecules and 202 isotoplc variants and 9 triatomic molecules and 119 isotopic variants. $\mathrm{J}-\mathrm{L}$. Tassoul discussed the problem of finding a rotation law and a circulation pattern so that radlative equilibrium prevails at every point in a star. He described how a baraclinic structure combined with a turbulent dissipative flow can lead to acceptable solutions.

Ruben considered the hydrodynamics of a rotating supernova. He explained that, even though the rotation energy is very much less than the collapse energy, there are very complicated motions. When the shock wave reaches the surface of the star, there is a strong meridional movement with a magnitude which is $10-20 \%$ of the 
radial velocity. Sugimoto said that, when considering problems in which substantial mass loss from a star is supposed to occur, it is necessary to take account of the energy involved in getting matter from the central regions to the surface so that mass loss can occur. He believed that this was very important in mass loss from neutron stars in $X$-ray burster models.

In addition to the Commission meetings, Commission 35 was concerned in three Jolnt Discussions on Solar Luminosity Variations, Evolution in 0id Stellar Populations in Galaxies and Mass Loss Phenomena. 

ATMOSPHERES STELLAIRES)

Report of the Meetings on August $23 \mathrm{rd}$ and 25 th

CHAIRMAN : G. Traving

SECRETARY: K. Kodaira

\section{Bussiness Meeting August 25th, 9:000 m}

I. COMMISSION ACTIVITIES

The President outlined the activities of the Commission in the period following the 17 th General Assembly.

II. MEMBERSHIP

a) DECEASED: Heidman, N.; Myerscough, V.P.

b) VOLUNTARY RESIGNATIONS: Fischel, D.; Thompson, R.I;

Unsc̈ld, A.

c) The president reported the names of those members, who

failed to confirm their membership.

d) The membership of the following new members was approved:

Crivellari, L.; Cannon, C.J.; Dupree, A.K.; Erikson, K.;

Froeschlé, C.; Haisch, B.M.; Hartmann, L.W.; Heaslev, J.N.;

Hubeny, I.; Koester, D.; Mnatsakanian, M.; Nagirner, D.I.;

Rachkovsky, D.N.; Rutten, R.J.; Simon, T.; Stencel, R.E.;

Vardavas, I.M.; Weber, S.V.; Wilson, S.J.; Yanovitsky, E.G..

III. NOMINATION OF PRESIDENT AND VICEPRESIDENT

The nomination of $\mathrm{B}$. Gustafsson as President and of $\mathrm{K}$. Kodaira as Vice-President was approved unanimously.

IV. ORGANIZING COMMITTEE

The following names for the new Organizing Committee were approved: Gray, D.; Hearn, A.G.; Kalkofen, W.; Kudritzki, R.; Mihalas, D.; Sapar, A.; Seaton, M.; Traving, G.; Underhill, A.B..

\section{FUTURE ACTIVITIES}

Plans for future activities of the Commission were discussed, but no decisions taken. The business meeting adjourned at 10:05 am.

\section{SCIENTIFIC SESSIONS}

1) JOINT SESSION (with Commissions 14 and 29): August 23rd, 8:30 am The minutes of this session will be published elswhere.

2) MEETING: August $23 \mathrm{rd}, 3: 15 \mathrm{~cm}$

Radiative Transfer

Chairman: W. Kalkofen

Introduction to papers on transfer in spherical atmospheres:

W. Kalkofen

Papers Presented:

(1) Construction of Models: R. Wehrse

(ii) Discrete Space Theory: A. Peraiah 
Introduction to papers on fast methods of solving the transfer equation: W. Kalkofen

Papers Presented:

(i) Probabilistic Radiative Transfer: R. Canfield

(ii) Use of Approximate Lambda-Operators: G. Scharmer

(iii) An Integral Enuation Method: P. Ulmschneider

The material presented at this session together with further papers on transfer in spherical media and on fast methods are to be published in a forthcoming book on "Methods in Radiative Transfer". The expected publication date is 1983.

3) MEETING: August $25 \mathrm{th}, 3: 15 \mathrm{pm}$

The impact of new observational data on the theory of stellar atmospheres.

Chairman: G. Traving

Papers Presented:

(i) The Impact of UV-Observations on the Theory of Late-Type Stellar Atmospheres: D. Reimers (presented by L. Hartmann)

(ii) The Impact of X-Ray Observations: A.K. Dupree (presented by $w$. Kalkofen)

(iii) The Impact of UV-Observations on the Theory of Early-Type Stellar Atmospheres: A.B. Underhill

The papers presented in this session form part of the report of Commission 36 which has been published in the "Transactions IAU Vol. $18 \mathrm{~A} ", \mathrm{pg} .479$. 
In addition to the Commission sessions described below, the Commission also participated in the following:

1. Jolnt Discussion II: Evolution in old stellar populations in galaxies (chairman: G. Lyngå)

2. Joint Discussion VII: Mass-loss phenomena (chairman: A.G. Hearn)

3. Session 251: Photometry in crowded fields (chairman: A. Penny)

4. Session 291: Systematic effects in abundance determinations for metal-poor stars (chairman: W.K. Bonsack)

Reports of the Joint Discussions will be found in Highlights of Astronomy, reports of Session 251 will be found in this volume in the proceedings of Commission 25 and reports of Session 291 are being printed in the Publications of the Astronomical Society of the Pacific.

\section{August 1982}

\section{BUSINESS SESSION}

1. The President summarised the changes in the membership of the Commission, and the following new organising committee was elected to serve for the three-year perfod 1982-85:
K.C. Freeman
(President)
G.L.H. Harris
D.C. Heggie
(V1ce-president)
G. Lyngå
(Past President)
P.E. N1ssen
C.A. P1lachovski
G.N. Salukvadze

2. A brlef report was given of IAU Colloquium No. 68, "Astrophysical Parameters for Globular Clusters" (Schenectady, New York, U.S.A., October $8-10,1981$ ), and the President Ifsted a number of other meetings which the Commission had also decided to support. It was decided to support the proposal by R.M. Humphreys for a meeting on "Luminous Stars, Associations and Galaxles", tentatively planned for autumn, 1984, in Europe.

3. The meeting discussed the following proposal for the numbering of stars in clusters, which was prepared by a Working Group consisting of A. Moffat (chairman), G. Lyngå, J.-C. Mermilliad, and S. van den Bergh. 
Proposal:

(1) All star cluster investigators should always publish finding charts that unambiguously indicate the stars observed (preferably as in (2) below), if not given explicitly elsewhere. Even the measure of only a few stars justifies a chart. The chart should be labelled " $N$ " at the top for north and "E" at the left for east with a line segment at the edge showing a simple number of arc units on the sky. Editors of appropriate fournals should be urged to insist that authors comply.

(2) Numbering should be made in a systematic fashion. The preferred, most flexible way is to use $X Y$ coordinates in minutes of arc, centered on some arbitrary but precisely defined star or other position near the cluster centre and aligned so that $X, Y$ are parallel to and $g 0$ in the same sense as RA and Dec, respectively, for 1950.0. Once the origin has been chosen it must remain the same for all future work on a given cluster. An approprlate level of precision for the tabulated $X, Y$ values should be adopted in order to avoid ambiguities. To facilitate easy use, the charts should indicate the $X, Y$ axes with tic marks at intervals of one arc minute or better, depending on the degree of concentration of the cluster. The scale should nominally be $l^{\prime}$ per $\mathrm{cm}\left(=6^{\prime \prime}\right.$ per $\left.\mathrm{mm}\right)$ or a simple multiple thereof. Once one good chart is published in this way for a given cluster, subsequent papers on the same cluster can, if appropriate, refer to it by $X, Y$ coordinates for identification of stars, without having to make a new chart. Cross-identifications to old numbering systems should be supplied.

(3) A photographic atlas containing charts of all existing Galactic open and globular star clusters should be made by some central body (e.g. CDS) as a basis for (2). The charts would be left blank except for the addition of $\mathrm{X}, \mathrm{Y}$ axes and tic marks. J.-C. Mermilliod has indicated he might supervise the undertaking of this task. Plates for Northern clusters will be obtained with the CERGA Schmidt telescope by Dr Heudier.

In the discussion that followed, J.-C. Mermilliod exhibited an example of a suitable chart prepared from a Schmidt plate, though how the atlas of charts should be published, and what the limiting magnitude should be, had not been settled. The atlas would cover perhaps 200 or so of the most frequently studied Galactic open and globular clusters. Astronomers working to fainter magnitudes than the chart limit, could prepare their own charts and send copies to the CDS (Centre de Données Stellaires) at Strasbourg. A suitable model for such charts is the well-used catalogue containing UBV magnitudes of stars in clusters by Hoag et al. (1961, Publ. U.S. Naval Obs. 17, part 7). The proposal of the Working Group was accepted.

4. B. Balázs reported on the status of the Catalogue of Star Clusters and Associations, and the problems caused by the exponential growth of 11terature in this field. By 1983 it was hoped that data published up to 1976 would be incorporated, and that the backlog would disappear by 1987 . To facilitate this, Dr Balázs repeated his appeal at Grenoble in 1976, i.e. "recognising the importance of the Catalogue of Star Clusters and Associations to all astronomers, Commission 37 urges all authors to send coples of their papers and these objects to the Editors of the Catalogue, B. Balazs or J. Ruprecht". 
G. Lynga stated that the Lund-Strasbourg computer-based catalogue of open cluster data would be updated in 1983. J.C. Mermilliod reported the addition of new UBV data for new clusters to his catalogue $(27.153 .023)$, and A.G. Davis Philip stated that he and R.E. White were engaged on a new edition of an atlas of colour-magnitude dlagrams of globular clusters.

\section{SCIENTIFIC SESSION}

This consisted of a poster session followed by a discussion supervised by the chafrmen.

1. Open Clusters (cha1rman: P.E. Nissen)

The following poster papers were displayed:

R. Cayre1, G. Cayre1 and B. Campbe11: New Observational Study of Lithium depletion along the main sequence of the Hyades.

Observations made at the coude focus of the Canada-France-Hawaii Telescope have allowed us to establish that the equivalent width of the 11thium resonance doublet at $6707 \AA$ decreases from $79 \mathrm{~m} \AA$ at spectral type GlV down to less than $2 \mathrm{~mA}$ at spectral type $\mathrm{K} 1 \mathrm{~V}$. This represents a depletion by a factor one hundred over this range.

A.M. Jakobsen: Differential Ages in Open Clusters.

A relationship between uvby, $\mathrm{H} \beta$ Strömgren photometric observations and the ages and masses of B-type stars has been established. In some open clusters an age spread from 10 million to 100 million years between the early (h1gh mass) and the late (low mass) B-type stars is indicated. NGC $457,663,1893,1960,2264,6910, \mathrm{~h}+\chi$ Per and stock 2 are extensively observed.

M.L. Roth: Ages of Open Clusters (a Catalogue).

A.R. Upgren and E.W. Weis: Photometry and luminosity function of the Hyades.

D.A. VandenBerg and T.J. Bridges: Zero-age main sequences applied to the Plelades, Praesepe, and Hyades.

Zhao Jun-L1ang: Determination of membership in open clusters by means of statitistical decision theory.

2. Globular Clusters (chairman: J. Mould)

The following poster papers were displayed:

G. Alcaino: NGC 6256: Definftively a Galactic globular cluster.

G. Alcaino: The globular cluster NGC 6544 .

G. Alcaino and W. Liller: Main sequence photometry for globular clusters.

A. Ardeberg, H. Lindgren and P.E. Nissen: Metal abundance and age of NGC 6397 from uvby- $\beta$ photometry of turn-off stars. 
A. Chleffi and F. D'Antona: CNO self-pollution in globular clusters: Evolutionary effects.

Making the working hypothesis that presently evolving stars in globular clusters have been polluted in $C N$ rich matter in the first epoch of the life of the cluster, we compute the evolution of stratifled stars, in which the external layers are $C N$ enriched. These stars evolve very rapidly when the hydrogen burning shell crosses the chemical discontinuity left by convection.

J.E. Hesser, W.E. Harris and B. Atwood: Photometry of globular clusters with the CTIO SIT Vidicon.

A color-magnitude diagram reaching $M_{V} \sim+9$ mag. has been constructed for NGC $104=47$ Tuc. It shows excellent agreement in position and slope with the theoretical tracks computed by D.A. VandenBerg for $Y=0.2, Z=0.006$ $([\mathrm{Fe} / \mathrm{H}]=-0.5), \alpha=1.65$, and $15 \leq \tau_{g} \leq 18$. A preliminary $\underline{\mathrm{V}}$ magnitude luminosity function for NGC 2257 in the LMC confirms Stryker's identification of the location of its turnoff and supports her contention that it is as old as M92.

W. Liller and G. Alcaino: A search for globular clusters around the edge-on spiral galaxies of the Sculptor Group NGC 55 and NGC 253.

T. Lloyd-Evans: MS stars in $\omega$ Centauri.

S.J. Shawl and J.E. Hesser: Radial velocities from integrated 11ght observations of globular cluster $\mathrm{H \alpha}$ absorption lines.

Radial velocities of $\sim 5 \mathrm{~km} \mathrm{~s} \mathrm{~s}^{-1}$ precision determined with the CTIO single-étalon Fabry-Perot interferometer for $\sim 85$ galactic globular clusters are generally in good agreement with those in Webbink's compilation (Astrophys. J. Supp1. 45, 259, 1981). Results we reported at IAU Coll. 68 from $120 \AA \mathrm{mm}^{-1}$ image-tube spectra for NGC $6171,6528,6624$, 6652,6681 and 6723 are confirmed by the higher accuracy F-P measurements.

\section{August 1982}

\section{SCIENTIFIC SESSION: CLUSTER CORES (chairman: D.C. Heggie)}

The following reviews were presented:

D. Lynden-Be11: Theory of core evolution.

Lynden-Bell showed how self-similar models elucidated core collapse and re-expansion. At the moment of final core collaps the density is of the form $\rho_{\alpha} \mathrm{r}^{-\alpha}$ with $\alpha=2.22$. After core collapse, binary formation and evolution at the centre provides the energy for a re-expansion with the central regions forming a singular almost 1sothermal sphere with $p$ o $\mathrm{r}^{-2}$. This law bends over to the $r^{-2.22}$ halo at a transition radius $r_{c}$ which Increases as

$$
\left|t-t_{0}\right|^{\frac{2}{6-\alpha}}
$$

In this re-expansion the characteristic scales follow the same law as the collapse phase (Lynden-Bell and Eggleton MNRAS 1980, 191, 483): 


$$
\begin{aligned}
& v_{c}^{2} \propto\left|t_{o}-t\right|^{(4-2 \alpha) /(6-\alpha)} ; M_{c} \propto\left|t_{o}-t\right|^{(6-2 \alpha) /(6-\alpha)} ; \\
& E_{c} \propto\left|t_{0}-t\right|^{2(5-2 \alpha) /(6-\alpha)} ; \rho_{c} \alpha\left|t_{o}-t\right|^{-2 \alpha /(6-\alpha)} .
\end{aligned}
$$

In the above $c$ refers to values at the edge of the core where

$-\delta \log \rho / \delta \log r$

changes from 2 towards 2.22 .

D.H. Martins: Surface photometry of globular cluster cores.

Recent results of surface and stellar photometry of several galactic globular cluster cores were reviewed. Most attention was given to those clusters presumed to contain X-ray sources or which possess excess central surface brightness. In particular, discussion centered around NGC 6397, $6440,6441,6624,6712$, and 7078. Data analysis techniques were reviewed with emphasis placed on useful developments in the area of image processing.

Selected references:

Aurfere, M. 1982. Astron. Astrophys. (in press).

Auriere, M., and Cordon1, J.-P. 1981. Astron. Astrophys. 100, 307.

Auriere, M., and Cordoni, J.-P. 1981. Astron. Astrophys. (Supp1. Ser.) 46 347.

Bahcall, N.A., Lasker, B.M., and Wamsteker, W. 1977. Astrophys. J.

(Letters) 213, L105.

Da'Costa, G.S. 1979. Astron. J. 84, 505.

Grindlay, J.E. 1981. IAU Colloq. No. 68, Astrophysical Parameters for

Globular Clusters, ed. A.G.D. Philip and D.S. Hayes.

Martins, D.H., Harvel, C.A., and Miller, D.H. 1980. Astron. J. 85, 521.

Martins, D.H. and Harve1, C.A. 1980. Astrophys. J. (Letters) 236, L77.

Martins, D.H., 1982. (in preparation).

The following poster papers were contributed:

A. Altamore, L. Angelett1, R. Capuzzo-Dolcetta and P. Giannone: UV observations of M5.

E. Bettwieser: Late stages of core collapse in globular clusters.

G.S. Bisnovatyi-Kogan, M.M. Romanova: Globular clusters and X-ray bursters.

D.C. Heggle: Post-collapse evolution of a gaseous cluster model.

S. Inagak1: The effects on binaries on the evolution of globular clusters. 
H.E. Kandrup: Dynamical friction in a mean field approximation: a summary.

J. Katz: On the stability of two-component cores of globular clusters.

G.E. Kron, L.H. Wasserman and A.V. Hewitt: Abnormalities in the brightness profiles of the core regions of some globular clusters.

A.P. Lightman: What's happened in the cores of globular clusters?

N.M. Spasova: Globular cluster cores - structure and analysis. 
COMMISSION 38 : EXCHANGE OF ASTRONOMERS - (ECHANGE DES ASTRONOMES )

Committee of the Executive Committee

Report of Meetings, August 18, 19, 24.

PRESIDENT : J. DELHAYE

SECRETARY : P.M. ROUTLY

1. First Meeting - August 18, 1982 .

President J. Delhaye called the first meeting of Commission 38 to order at 1100 hrs, Aug 18, 1982.

1.a Recording Secretary

At President Delhaye's request, Dr. P.M. Routly accepted the assignment of recording the minutes of the Commission's meetings during the course of the XVIIIth General Assembly.

1.b Members Present

The following members or observers, together with their countries of affiliation, were present at the August 18 th meeting : H.M. A1-Naimy, IRAQ; T.M. Borchkhadze, USSR; J. Delhaye, France; M.K. Hamid, ....; E.A. Muller, SWITZERLAND; P.M. Routly, USA; C.R. Tolbert,USA; F.B. Wood, USA.

1. c Agenda

Mr. Delhaye announced that there would be a Joint Meeting with Commission 46 on Aug 19 and a second meeting of Commission 38 on Aug 24. President Delhaye stated that the purpose of the Joint Meeting was to learn details of a new Visiting Lecturers Program which had been proposed to the Executive Committee by Commission 46 and had already been approved.

The following agenda to cover all meetings involving Commission 38 was agreed to by the attending members.

\section{AGENDA}

1. Report of the President

1.1 - The activities of the Comnission for the last three years.

1.2 - Critical remarks which have been made.

1.3 - Budget.

2. Open Problems

3. Joint Meeting with Commission 46, Aug 19 th

4. Election of Officers and Members of Commission 38 .

President Delhaye stated that he wished to discuss Items 1.1 and 1.3 immediately but defer Items 1.2, 2, and 4 to the secpnd meeting of Commission 38 on Aug 24 .

\section{1.d President's Report - Agenda Items $\neq 1.1$ and $\neq 1.3$}

President Delhaye distributed a report covering the years 1979-1982 which included a current listing of the members of Commission 38, a copy of the Guidelines defining the Exchange of Astronomers Program as adopted in 1980, Interim Reports $\neq 1$ and $\neq 2$ on the Commission's detailed operations dated June 1, 1980 and May 15, 1981, a final report to the Executive committee dated Nov 30,1981 , an addendum to the $f i-$ nal report covering the period Dec 1, 1981 to July 15, 1982, and a brief summary of finances. Between the Montreal General Assembly and July 15, 1982, a total of 35 applications were received, 22 grants were awarded, 8 applications were denied, 3 were withdrawn, and 2 are still pending. 
Total expenses to June 1st, 1982 amounted to approximately 61,319 SFR. Since the budget allocated to Commission 38 for the period 1979-82 was only 58,000 SFR, it was necessary for President Delhaye to approach the Executive Committee for supplementary funding. An additional sum of 10,000 SFR was authorized, raising the total budget to $68,000 \mathrm{SFR}$, permitting the operations of Commission 38 to balance.

While no formal action was needed or taken, the Committee Members in attendance endorsed President Delhaye's report and expressed the general feeling that the Exchange of Astronomers Program had been effectively and efficiently run during the past three years.

With regard to the future, President Delhaye reported that Comnission 38's budget for 1982-85 has again been set at 58,000 SFR by the Executive Committee. Assuming the continued financial health of the IAU, it was understood that the Executive Committee would consider an augmentation to this budget should the demands on Commission 38 require it.

There being no further business to discuss, President Delhaye adjourned the meeting at 12.30 hours.

\section{Joint Meeting with Commission 46 - August 19, 1982.}

Interested members of Commission 38 met with Commission 46 on August 19, 1982 at 11.00 hours to discuss the Education of Astronomers from Developing Countries and Plans for the Visiting Lecturers Program. Results of these deliberations are described in paragraph 3.c below.

3. Second Meeting - August 24, 1982.

President Delhaye called the second and final meeting of Commission 38 to order at 15.00 hours on August 24,1982 .

\section{3. a Members Present}

The following members or observers, together with their countries of affiliation, were present at the Aug 24 meeting :T.M. Borchkhadze, USSR; A.A. Boyarchuk, USSR; J. Delhaye, FRANCE; S. Ferraz Mello, BRAZIL; H.F. Haupt, AUSTRIA; K.C. Leung, USA; P.M. Routly, USA; W. Shougnan ....; F.G. Smith, UK; C.R. Tolbert, USA; D. Wentze1, USA; F.B. Wood, USA.

\section{3.b New Officers and Members of Commission 38 - Agenda Item $\neq 4$}

After careful examination and general discussion of the present composition of Commission 38, the following slate of officers and members for 1982-85 was proposed for ratification at the second session of the XVIII General Assembly :

President F.B. WOOD

University of Florida, Department of Astronomy, Thomas W. Bryant Bldg Gainesville, F1a 32611.

Vice President E.A. Muller Observatoire de Genève, Sauverny,

Organizing Committee

Members
Suisse.
A.A. Boyarchuk; J. Delhaye; D.A. MacRae; P.M. Routly; J. Sahade; F.G. Smith; C.R. Tolbert; H.H. Voigt; S.H. Ye. A.W. Al-Sabti; B.J. Bok; A. Florsch; G. Gordoli; H. Haupt; G. Keller; K.C. Leung; H. Li; M. Marik; S. Miyamoto; I.S. Nha; S.E. Okoye; A. Opolski; T.L. Page; A. Reiz; G. Ruben; E.V.P. Smith; G. Teleki; J.P. Wild.

3.c Joint Meeting with Commission 46 - Agenda $\neq 3$

As a result of the Joint Meeting held on Aug 19, the following actions were formally endorsed by Commission 38 at its Aug 24 meeting.

1. Commissions 38 and 46 adopted the report of the joint Working Group on the Education of Astronomers from developing countries. The Working Group, having completed its charge, was formally terminated. 
2. Commissions 38 and 46 resolved to establish a subcommittee to implement the recommendations of the Visiting Lecturers Program in the report of the Working Group and in Agenda item 13(d) 2 of the 18th General Assembly of the IAU. Initialy, the membership of the committee will be J. Delhaye (38), S. Ferraz-Mello (46), D.C. Wentzel (46, Chairman), and F.B. Wood (38). The membership will be subsequently expanded to ensure active participation from Developing Countries in its work, with advice and consent from the Organizing Committees of Commissions 38 and 46 .

It was agreed that the terms of reference for the subcommittee would be:

a. To seek individuals and their institutions willing to host a Visiting Lecturers Program.

b. To identify the local procedures to support the program; in particular, the local body with whom any future contrats should be made.

c. To outline a Visiting Lecturers Program agreeable to both host and subcomittee; at this time the subcommittee should identify several possible lecturers with experience in the field of the agreed programme, known experience of the developing world and preferably fluency in the local language. ( It is recognized that such an identification will not limit final selection.)

d. To submit, in light of $c$. above, a formal proposal to the IAU Executive Committee for a Visiting Lecturers Programme in a named Developing Country, to be based an a named host institution.

e. To submit at the time of d. above, the outline of a second programme, such that the General Secretary may seek support through a UNESCO,contract.

f. To establish, at the time of d. above, an advisory panel with experience of local conditions, developing countries and the type of programme envisaged, in order to advise the Executive Committee on the final selection of lecturers and on the articulation and oversight of the programme.

It was further agreed that the countries to be initially contacted with little or no astronomy, would be : Egypt, Indonesia, Kenya, Korea, Nigeria, Paraguay, Peru, Portugal, Sri Lanka, Thailand.

3.d Criticisms and Problems - Agenda Items $\neq 1.2$ and $\neq 2$.

President Delhaye reported that a few members on the Executive Committee had raised several questions critical of Commission 38 's operation.

a. Qualifications of Candidates. One of these questions had to do with assuring that candidates for Commission 38 grants were the best qualified available. It was suggested that Commission 38 have some way of intercomparing or evaluating candidates so that they might be ranked in some kind of order of merit or desirability.

The Commission members in attendance discussed this idea at some length and rejected it for the following reasons. First, it implies the existence of deadlines so that applications could be accumulated prior to the rating process. This contrasts:to the present procedure where applications are acted upon as they are received. It was felt that the "deadline approach" would require too much lead time to be responsive to the schedules of prospective applicants. As to the qualifications of applicants, it was pointed out that present procedures already require the approval, support, and endorsement of the candidate by responsible officials at both the Host and Home Institutions. To require further approval was felt to be an unnecessary additional complication and one that cast doubt on the competence and veracity of the applicant's endorsers.

b. Per Diem and Dependents. Another question that was raised concerned per diem and dependents. It was suggested that the Exchange of Astronomers Program cover per diem costs as well as the costs of dependents in lieu of travel in some cases and in addition to travel in others. Commission Members agreed that such support would be ideal but was not feasible within the framework of the present budget.

In summary, the Commission decided to recommend thet the present guidelines be left as they are and that the Exchange of Astronomers Program continues to be operated without substantial change from current operational procedures.

3.c Thanks and Adjournment.

President Delhaye thanked the Commission and its officers for their work and 
support over the past three years. He also extended best wishes to the new officers and expressed the hope of seeing everyone again at the next General Assembly in India in 1985. There being no further business to discuss, President Delhaye adjourned the' meeting at 16.45 hours.

P.M. ROUTLY, Secretary. 
Report of Meetings, 18, 19, 20, 21, 23, 24 and 25 August 1982

PRESIDENT: G. Swarup

SECRETARY: V.K. Kapahi

\section{Business Meet ings}

With the President, Govind Swarup, in the chair, business meetings of the Commission were held on 19 and 25 August. The following business was transacted.

\section{REPORT OF THE PRESIDENT}

The activities of the commission during the preceding three years were briefly summarised by the President. Although the radio astronomy service seems to have got a reasonable deal, on the whole, at the World Administrative Radio Conference (WARC) in 1979, we must continue our efforts to obtain adequate protection for the radio astronomy bands from harmful transmissions by other services.

The President thanked all the contributors to the triennial report which had been published by the IAU and was available at Patras. As in the previous report astrophysical results obtained through the radio technique were covered by several other appropriate Commissions. The Commission 40 report deals mainly with basic measurements, radio surveys, and new developments in radio telescopes and instrumentation.

During the three years the Commission cosponsored IAU Symposia 95-Pulsars; 97-Extragalactic Radio Sources: 100-Intermal Kinematics and Dynamics of Galaxies; 101-Supermova Remnants and their X-ray Emission: 104-Early Evolution of the Universe and its Present Stmucture; Colloquium 71-Activity of Red Dwarf Stars.

\section{LINES OF ASTROPHYSICAL IMPORTANCE}

Brian Robinson summarised the activities of the working group on the protection of radio spectral lines. Based on comments from several astronomers, the group recommended that the lines at $36.392 \mathrm{GHz}\left(\mathrm{HC}_{3} \mathrm{~N}\right.$ ) and $150.74 \mathrm{GHz}$ (nitric oxide) be deleted and several other lines be added to the 1979 list of astrophysically important lines (IAU Transactions Vol.XVII B). The new list included several lines above $275 \mathrm{GHz}$, a region of the spectrum pioneered by radio astronomers. Following the discussion that ensued, the WG met again on 24 August and the proposed changes to the 1979 list were again discussed in the 2nd business session. The revised new list of lines, approved by the Commission is shown in Table 1 in which the 1 ines below $275 \mathrm{GHz}$ are listed in order of increasing frequency while those above $275 \mathrm{GHz}$ are ranked in order of their foreseen astrophysical significance. The revised membership of the WG is: A. Baudry, R. Booth, L. Doherty, M. Morimoto, V. Pankonin, V. Radhakrishnan, B. Robinson (Chairman), R. Schilizzi, V. Slysh, B. Turner,

A. Webster and T. Wilson.

In view of the importance of obtaining official backing of IAU, a resolution (1isted in the Proceedings of the XVIIIth General Assembly) concerning protection from harmful interference was unanimously approved by the members.

\section{WORKING GROUP ON VLBI}

Ken Johnston proposed that a working group be formed to foster the interdisciplinary use of large steerable antennas for astrophysical, astrometric and geodetic research in Very Long Baseline Interferometry (VLBI). The proposal was well received by the members and a resolution recommending the formation of such a 
working group was approved. The resolution was later endorsed by the IAU General Assembly. The working group, whose present membership is K. Johnston (Chairman), S. Ananthakrishnan, D. Backer, E. Bajaja, R. Booth, N. Broten, M. Cohen, G. Grueff, D. Jauncey, P. Kaufmann, K. Kellermann, A. Kus, L. Matveyenko, M. Morimoto, G. Nicholson, I. Pauliny-Toth, R. Schilizzi, P. Wilkinson and S. Ye, will be concerned mainly with the collection and dissemination of information concerning the available facilities and instrumentation at different observatories, plans of experiments in astronomy and geodesy, and the need for symposia etc. with a view to encouraging international cooperation in the use of VLBI.

\section{RADIO SOURCE NOMENCLATURE}

A resolution recommending a system of naming radio sources by positions (the present IAU system) referred to epoch $J 2000.0$ and renaming of sources referred to epoch B 1950.0 was proposed by Bernie Burke. As no consensus could be reached at the meeting, it was agreed to form an ad-hoc working group that would discuss the issue in some detail and come up with a recommendation at the second business session. The working group; composed of B. Burke (Chairman), J. Baldwin, H. Dickel, K. Johnston, V. Kapahi, B. Mills, R. Strom and G. Westerhout met on 23 rd August and concluded that it was not prepared to recommend any changes at this time. A reconvocation of the group might be appropriate at the next General Assembly of the IAU.

\section{PULSAR TIME SCALE}

N. Kardashev on behalf of V.G. II'in et al. proposed the creation of a new time scale, to be called Pulsar Time Scale, based on radio pulses from PSR $0834+16$ as the main reference and $0950+08$ and $1919+21$ as other references. According to them, such a time scale would be more precise and stable than Universal Time and Ephemeris Time Scales. After some discussion it was agreed that an ad-hoc committee consisting of $R$. Manchester, D. Backer and A. Lyne should consider the merits of the proposal in detail and report their findings at the second meeting of the Commission. The gist of the Committee's recommendations was as follows. Any such proposal should be made jointly with Commission 31. Although a Pulsar Time Scale would be useful on $1 y$ for long time intervals it remained to be shown that the precision achieved would be comparable to that defined by TAI. More than 3 pulsars would be required to take care of possible large period variations in one or more pulsars. Accurate positions and proper motions will need to be known. There are difficulties in defining a standard pulse template; in general this template will vary with frequency. Dispersion Measures will also need to be accurately known. A moderately large antenna will be required to obtain the necessary sensitivity.

A decision on the proposal was therefore deferred.

\section{IUCAF AND CCIR ACTIVITIES}

Gart Westerhout summarized the activities of IUCAF which is now concerned with the actions to be taken in the post-WARC period. The impact of WARC-79 on Radio Astronomy has been well summarized in a paper published by V. Fankonin and R.M. Price (IEEE Trans. on Electromagnetic Compatibility, 1981, Vol. EMC-23, p.308). IUCAF will watch closely the preparations for the forthcoming WARC (1985) dealing with the efficient use of the geostationary orbit.

A brief report of the activities of CCIR Study Group-2 since WARC-79 was presented by Lorne Doherty. The Study Group met in Geneva in 1980 and 1981 and the Plenary Assembly was held in February 1982. A new report of CCIR, containing the work done at these meetings, will be published later this year (Recommendations and Reports of the CCIR, XV Plenary Assembly, Geneva 1982, Vo1. II - Space Research and Radio Astronomy). There has been a major reorganization of texts dealing with radio astronomy, as a consequence of the results of WARC -79 and the addition of new technical material. 
The Study Group-2 has formed a working party to prepare technical material for the 1985 Space WARC. Consideration of emissions from space, particularly out of band emissions, will be an important subject of discussion in the coming years. Other important areas of interest are preferred frequencies for spectral 1 ine observations, harmful interference criteria for synthesis and VLBI arrays, and various sharing problems with other services. Radio astronomers involved with CCIR work would welcome assistance from other colleagues in these areas.

\section{REPRESENTATION IN CCIR AND IUCAF}

IAU has two representatives in CCIR and IUCAF. The commission chose $J$. Whit eoak as one of the new CCIR representatives in place of B. Robinson. L. Doherty will continue to be the other representative. For IUCAF, G. Swarup will replace G. Westerhout, while R. Schilizzi will continue to be the other representative.

\section{TRANSMISSIONS AT $38 \mathrm{MHZ}$}

A suggestion was made by Roger Jennison that since current observations at $38 \mathrm{MHz}$ suffer severe interference from long distance propagation of transmissions, particularly from the USA and the USSR, a resolution should be passed recommending that every effort be exerted to remove all transmissions from the $38 \mathrm{MHz}$ band. Several members were of the opinion, however, that any such resolution would have little effect at present, and it would be preferable to take up the matter first through the concerned national administrations. After considerable discussion the proposed resolution was 'reluctant ly' withdrawn.

\section{INTERNATIONAL HALLEY WATCH}

William Irwine made a brief presentation on the aims of the 'International Halley Watch' project and stressing the importance of coordinated radio observations of the comet in the coming years, with the most sensitive instruments. Those planning to make observations, both in the continuum and in molecular lines should get in touch with him at the Department of Physics and Astronomy, University of Massachusetts, Amherst, Massachusetts 01003.

\section{$X$. NEW OFF ICERS AND ORGANIZING COMMITTEE}

The Commission unanimously approved the following slate suggested by the outgoing Organizing Committee: President: K. Kellermann, Vice President: J. Baldwin Organizing Committee: J. Ables, N. Broten, G. Dulk, B. Höglund, N. Kardashev, M. Morimoto, S. Okoye, G. Setti, R. Strom, G. Swarup, L. Weliachew, T. Wilson and S. Wang.

\section{$X I$. NEW MEMBERS}

Proposal to add 67 new members whose names had been put up on the notice boards earlier was approved by the Commission. The total membership of the Commission has now almost reached the 500 mark.

\section{MISCEL LANEOUS}

Paul Wild's announcement that the news of the Australian Government's decision to fund the proposed Australian Synthesis radio telescope had just reached Patras was greeted by members with applause and jubilation.

The second business meeting closed with Ken Kellermann, the new President, proposing a vote of thanks to all those responsible for organizing Commission activities in Patras and with a request to members to let him have their views regarding any changes they would like to see in the Commission's activities in future. 
Scientific Meetings

\section{JOINT DISCUSSIONS}

Commission 40 cosponsored two Joint Discussions. Active Galactic Nuclei, with Commissions 28 and 48 on 18 August and Extragalactic Distance Scale and the Hubble Constant with Commissions $25,28,45,47$ and 48 on 23 August. The transactions of these meetings will be published by the IAU in 'Highlights of Astronomy'.

II. JOINT MEETINGS

Commission 40 participated in as many as five Joint Meetings.

(i) Early Years of Radio Astronomy - with Commission 41 on 19 August.

(ii) Solar Maximm Year - with Commissions 10,12 and 44 on 20 August.

(iii) The Violent Interstellar Medium - with Commissions 34 and 48 on 20 August.

The details of the above meetings may be found in the reports of other Cominissions and/or in 'Highlight.s of Astronomy'.

(iv) A Joint Meeting with Commissions 33 and 34 entitled Recent Results: Galactic and mm-wave was held on 24 August. In the first session of this meeting, chaired by G. Swarup, the following papers were presented.

F.G. Smith: Pulsar Results from Jodrel1 Bank

V. Radhakrishnan: Recent Pulsar work at RRI, Bangalore

D.J. Smith: Electric Field in Pulsar Magnetospheres

R.N. Manchester: Pulsar Search in Supernova Remnants

T. Velusamy: (i) Pulsar Search at Ooty, (ii) Interstellar Scattering towards the Inner Galaxy

B.Y. Mills: Supernova Remnants in the Magellanic Clouds

R.C. Jennison: A Structural Anomaly in Cas A

R.D. Ekers: High Resolution Continuum and Recombination Line Radio Observations of the Galactic Centre.

The second session was chaired by $V$. Radhakrishnan and the following papers presented.

K.J. Johnston: Recent VLA Results on HII Regions

V. Hughes: VLA Observations of Cep A

F. Churchwe11: The Kinetic Temperature Profiles of Sgr B2, Orion and DR21

I. Kazes: Possible Relation between HI Gas and Molecules

R.P. Norris: MERLIN OH Maps

C. Heiles: (i) Recent mm-wave Interferometric Results from Hat Creek, (ii) Magnetic Field Strengths in two Types of Region

H.R. Dickel: Observations of HCN in DR21 with the Hat Creek Interferometer

L.E.B.Johansson: Spectral Scan of Orion A and IRC+10216

B.J. Robinson: Southern Galactic Plane CO Survey

J. Guibert: ${ }^{13} \mathrm{CO}$ Observations toward the Second Galactic Quadrant

B. Irwine: Discovery of Interstellar $\mathrm{HOC}^{+}$

N.J. Evans II: Models of Molecular Clouds: Multi-transition Studies of $\mathrm{CS}$ and $\mathrm{H}_{2} \mathrm{CO}$

(v) A Joint Meeting with Commission 28 entitled Beams and Jets in Extragalactic Sources was held on 25 August with R.D. Ekers in the chair. The following talks were given. 
R.A. Laing: Large-Scale Radio Jets (review)

P.N. Wilkinson: Small-Scale Radio Jets (review)

L. B. Baath: VLBI monitoring of BL Lacs

G. Swarup: Jets in Quasars 3C9 and 3C280.1

H. Murdoch: Precessing Jets in 2300-189

J. van Gorkom: Jets in Edge-on Spirals?

G.K. Miley: Optical Studies of Jets (review)

W. van Breugel: Optical Observations of 3C293

K.J. Johnston: SS433-Recent Results (review)

P.A.G. Scheuer: Problems in Jet Physics (review)

R. Sanders: Reconfinement of Jets

The procedings of the above session will be published in 'Highlights of Astronomy'.

\section{I. COMMISSION MEETINGS}

i) Recent Results: Extragalactic. The talks given at this session (24 August), chaired by V.K. Kapahi, were:

B.F. Burke: Gravitational lenses: Probes of the dark matter

M.V. Gorenstein: High sensitivity VLBI maps of $0957+561 \mathrm{~A}, \mathrm{~B}$

D. Walsh: Mass distribution of the gravitational lens $0957+561$

R.J. Davis: MERLIN observations of 3C273

R.T. Schilizzi: Large scale radio structure of superluminal sources

K.J. Johnston: Recent results on BL Lac sources

J.E. Baldwin: Structure and spectra of giant radio galaxies

V.K. Kapahi: Radio and optical axes of elliptical galaxies

F.G. Smith: Is the Universe rotating?

D.C. Backer: VLBI at $89 \mathrm{GHz}$

S.H. Ye: The Shanghai-Effelsberg VLBI experiment

L. Weliachew: Neutral gas kinematics and $\mathrm{OH}$ masers in the central regions of $\mathrm{M} 82$ J.M. van der Hulst: Radio supernovae

ii) Reports from Observatories. In this session, whose chairman was K.I. Kellermann, the following talks were given.

J. Guibert: Technical developments in Paris/Meudon observatories

G.S. Downs: Radio astronomy with the NASA Deep Space Network

L.J. Malkamaki: An actively stabilized acousto-optical spectrometer

G. Westerhout: USNO-Green Bank astrometry project

M. Morimoto: The Nobeyama $45 \mathrm{~m}$ radio telescope

A. Baudry: Programs with the Bordeaux mm-telescope

R.N. Manchester: Radio astronomy at CSIRO

M.R. Kundu: Recent results from Clark Lake

P. Kaufmann: Latest results from Itupetinga

R.T. Schilizzi: Report from the Netherlands Foundation for Radio Astronomy

N.W. Broten: The proposed Canadian Long Baseline Array

E.E. Baart: The Rhodes-Hartebeesthoek $2.3 \mathrm{GHz}$ survey

P. Thomasson: First two years of MERLIN

R.D. Ekers: Status of the VLA and VLB array

R. Booth: Recent results from Onsala

B. Hooghout: The French/German mu-telescopes

A. Kus: New developments in Torun 
TABLE 1. RADIO FREQUENCY LINES OF THE GREATEST ASTROPHYSICAL SIGNIFICANCE

\begin{tabular}{|c|c|c|}
\hline Line & $\begin{array}{l}\text { Frequency } \\
\quad(\mathrm{GHz})\end{array}$ & Line \\
\hline
\end{tabular}

I. Lines below $275 \mathrm{GHz}$

\begin{tabular}{|c|c|c|c|c|c|c|}
\hline $\mathrm{D}$ & dent er ium & 0.3274 & & $\mathrm{C}_{2} \mathrm{H}$ & ethynyl radical & $87.317 *$ \\
\hline $\mathrm{H}$ & hydrogen & 1.4204 & & $\mathrm{HCN}$ & hydrogen cyanide & 88.632 \\
\hline $\mathrm{OH}$ & hydroxyl & 1.6122 & & $\mathrm{HCO}^{+}$ & formyl ion & 89.189 \\
\hline $\mathrm{OH}$ & hydroxyl & 1.6654 & & HNC & H. isocyanide & $90.664 *$ \\
\hline $\mathrm{OH}$ & hydroxyl & 1.6674 & & $\mathrm{~N}_{2} \mathrm{H}^{+}$ & & 93.17 \\
\hline $\mathrm{OH}$ & hydroxyl & 1.7205 & & $\mathrm{CS}$ & C. monosulphide & 97.981 \\
\hline $\mathrm{CH}$ & $\mathrm{CH}$ radical & 3.2638 & & ${ }^{12} \mathrm{C}^{18} \mathrm{O}$ & carbon monoxide & 109.782 \\
\hline $\mathrm{CH}$ & Cil radical & 3.3355 & & ${ }^{13} \mathrm{C}^{16} \mathrm{O}$ & carbon monoxide & 110.201 \\
\hline $\mathrm{CH}$ & CH radical & 3.3492 & & $\mathrm{CO}$ & carbon monoxide & 115.271 \\
\hline $\mathrm{H}_{2} \mathrm{CO}$ & forma ld ehyde & 4.8297 & & $\mathrm{H}_{2} \mathrm{CO}$ & formald ehyde & 140.840 \\
\hline $\mathrm{H}_{2} \mathrm{CO}$ & forma ld ehyde & 14.488 & * & $\mathrm{CS}$ & $(J=3 \rightarrow 2)$ & 146.969 * \\
\hline $\mathrm{H}_{2} \mathrm{O}$ & water & 22.235 & & $\mathrm{H}_{2} \mathrm{CO}$ & formald ehyde & 150.498 \\
\hline $\mathrm{NH}_{3}$ & ammonia & 23.694 & & $\mathrm{H}_{2} \mathrm{O}$ & water $\left(3_{13} \rightarrow 2_{20}\right)$ & $183.310 *$ \\
\hline $\mathrm{NH}_{3}$ & ammonia & 23.723 & & $\mathrm{cs}$ & $(J=4 \rightarrow 3)$ & 195.962 * \\
\hline $\mathrm{NH}_{3}$ & ammonia & 23.870 & & ${ }^{12} \mathrm{C}^{18} \mathrm{O}$ & $(J=2+1)$ & 219.560 \\
\hline SiO & silicon monoxide & 42.821 & & ${ }^{13} \mathrm{C}^{16} \mathrm{O}$ & $(\mathrm{J}=2+1)$ & 220.399 \\
\hline SiO & silicon monoxide & 43.122 & & $\mathrm{CO}$ & $(J=2+1)$ & 230.538 \\
\hline CS & C. monosulphide & 48.991 & & $\mathrm{CS}$ & $(J=5+4)$ & $244.953 *$ \\
\hline $\mathrm{DCO}^{+}$ & formy 1 ion & 72.039 & * & $\mathrm{HCN}$ & $(J=3+2)$ & 265.886 * \\
\hline SiO & silicon monoxide & 86.243 & & $\mathrm{HCO}^{+}$ & $(J=3+2)$ & $267.557 \star$ \\
\hline $\mathrm{H}^{13} \mathrm{CO}$ & formy 1 ion & 86.754 & * & HNC & $(\mathrm{J}=3+2)$ & $271.981 *$ \\
\hline
\end{tabular}

II. Lines above $275 \mathrm{GHz}$ *

$\begin{array}{llllll}\mathrm{C} & \left({ }^{3} \mathrm{P}_{1} \rightarrow{ }^{3} \mathrm{P}_{0}\right) & 492.162 & \mathrm{HCN} & (\mathrm{J}=4 \rightarrow 3) & 354.484 \\ \mathrm{CO} & (\mathrm{J}=3 \rightarrow 2) & 345.796 & \mathrm{HCO}^{+} & (\mathrm{J}=4 \rightarrow 3) & 356.734 \\ \mathrm{C} 0 & (\mathrm{~J}=4 \rightarrow 3) & 461.041 & \mathrm{~N}_{2} \mathrm{H}^{+} & (\mathrm{J}=3 \rightarrow 2) & 279.511 \\ \mathrm{CO} & (\mathrm{J}=6 \rightarrow 5) & 691.473 & \mathrm{~N}_{2} \mathrm{H}^{+} & (\mathrm{J}=4 \rightarrow 3) & 372.672 \\ { }^{13} \mathrm{C}^{16} \mathrm{O}(\mathrm{J}=3 \rightarrow 2) & 330.587 & \mathrm{NH}_{3} & \left(1_{\mathrm{O}}+\mathrm{O}_{\mathrm{O}}\right) & 572.498 \\ { }^{13} \mathrm{C}^{16} \mathrm{O}(\mathrm{J}=4+3) & 440.765 & \mathrm{H}_{2} \mathrm{O} & \left(1_{10^{+}} 1_{21}\right) & 556.936 \\ { }^{12} \mathrm{C}^{18} \mathrm{O}(\mathrm{J}=3 \rightarrow 2) & 329.330 & \mathrm{H}_{2} \mathrm{O} & \left(4_{14}+321\right) & 380.197 \\ { }^{12} \mathrm{C}^{18} \mathrm{O}(\mathrm{J}=4 \rightarrow 3) & 439.088 & & & \end{array}$

Note: Lines marked with an asterisk are additions to the 1979 1ist. Lines at $36.392 \mathrm{GHz}\left(\mathrm{HC}_{3} \mathrm{~N}\right)$ and $150.74 \mathrm{GHz}(\mathrm{Nitr}$ ic Oxide) have been deleted from the 1979 list. 


\section{August 1982}

\section{THE USE OF HISTORICAL RECORDS IN ASTRONOMICAL RESEARCH}

Nearly fifty astronomers gathered to hear papers discussing areas of astronomical research in which evidence is drawn from historical records. A primary purpose of the session was to alert researchers to the problems involved in assessing the historical record -- its reliability, completeness, interpretation, etc. Speakers also hoped to interest historians who might lead them to historical data hitherto unrecognized.

F. Richard Stephenson spoke of supernovae and ancient eclipse records, and reported that lunar eclipses now seem to offer the strongest evidence for determining the slowing of Earth's rotation during the past 2500 years. R. Nadal discussed the first part of an investigation of the Hipparchus commentary on Eudoxus and Aratus, which should eventually shed light on the origins of the Ptolemaic star catalogue. Samuel Goldstein described his analysis of thirteen tables in the Almagest, which has led him to the controversial conclusion that these tables antedate Ptolemy's work by several centuries. Tao Kiang reported on the ongoing Chinese searches for astronomical phenomena in provincial histories. A paper read on behalf of S.R. Malin and D. Gubbins urged that historians keep on the look-out for geomagnetic data in pre-1350 records. Finally, John Eddy gave a general summary of astronomical data in a variety of early records, including some from American Indians.

\section{August 1982}

\section{JOINT MEETING WITH COMMISSION 40 ON THE EARLY HISTORY OF RADIO ASTRONOMY}

This crowded session was arranged by a committee set up jointly with Commission 40 (radio astronomy), and commemorated the fiftieth anniversary of Kar1 Jansky's detection of cosmic radio signals. The speakers were: Robert Hanbury Brown, "Early radio astronomy at Jodrell Bank"; Peter Scheuer, "The development of aperture synthesis"; Bernard Mills, "The first radio source surveys and the $\log \mathrm{N}-\log \mathrm{S}$ relationship"; William McCrea, "The influence of radio astronomy on cosmology"; Fred Haddock, "The development of radio astronomy in the United States"; F. Graham Smith, "Early radio source work at Cambridge"; and W.T. Sullivan, "The discovery of the $21-\mathrm{cm}$ hydrogen line". Written versions of what were often very informal presentations are to be included in work to be published by Cambridge University Press.

\section{August 1982}

\section{BUSINESS SESSION}

Members stood for a few moments to honour the memory of members recently deceased: Joseph Ashbrook, Arthur Beer, Maria-Luisa Righini-Bonelli, Willy Hartner, llenri Miche1, B.E. Tumanjan, and F. Zagar. O. Pedersen (Denmark) was proposed as 
President for 1982-85 and J.A. Eddy (USA) as Vice-President. H. Eelsalu (USSR), E.G. Forbes (UK, representing IUHPS), M.A. Hoskin (UK), J.P. Verdet (France), and $K$. Yabuti (Japan) were elected as members of the Organizing Committee. It was noted with pleasure that E. Poulle (France) and C. Wilson (USA), hitherto consulting members of Commission 41 , were to be elected full members of IAU. R.L. Bishop (Canada), Z.-G. Chen (China), J.B. Carlson (USA), J. Darius (UK), P.S. Florides (Ireland), G.M. Idlis (USSR), C.J. Peterson (USA), N.A. Porter (Ireland), Z.-Z. Xi (China) and Q.-M. Zhao (China), together with E. Poulle and C. Wilson, were elected members of Commission 41.

As all consulting members lapse at each General Assembly, the following were elected consulting members for 1982-85: A. Aaboe, W.B. Ashworth, A.F. Aveni, E. Badolati, J.A. Bennett, R. Billard, P.G. Bulgakov, D.H. DeVorkin, A.I. Eremeeva, B.R. Goldstein, N.S. Hetherington, E.S. Kennedy, D.A. King, I.A. Klimishin, H.G. Korber, P. Kunitzsch, H. Labat, N.B. Lavrova, F.R. Maddison, Y. Maeyama, R. Mercier, N.I. Nevskaya, D.J. Price, B.A. Rosenfeld, G. Rosinska, G. Saliba, J. Samso, A.R. Serban, R.W. Smith, Z. Sokolovskaya, G.J. Toomer, G. L'E. Turner, A. Van Helden, J. Vernet Gines, B.L. van der Waerden, D.W. Waters, S.R. Weart, R.S. Westman, and D.T. Whiteside.

Part of the third session was devoted to a discussion, introduced by 01 af Pedersen on the basis of replies to a questionnaire, concerning tools needed by the historian of astronomy. There was support for proposals for a number of publications, amongst them: The Alfonsine Tables together with an explanatory appendix; the Almagest in the Latin translation of Gerard of Cremona; a facsimile edition of a medieval collection of astronomical manuscripts; a glossary of Latin astronomical terms; and algorithms to be used with computers. The Commission voted to have the Organizing Committce set up a working group to promote the publication of suitable facsimile editions, and the Committee was also instructed to work to establish a central catalogue of observations made before 1600 .

Announcements were made concerning a number of projects. Hoskin reported that the General history of astronomy, which is being published by Cambridge University Press under the auspices of IAU and IUHPS; will now have each of the four volumes divided into either two or three parts, and that part 1 of vol. iv is now in the press. It is hoped that two parts will be published each year. Pedersen announced that the manuscripts, notes and correspondence of Ejnar Hertzsprung, now in the History of Science Department of Aarhus University, were available on microfiche, and Hoskin reported that plans were well advanced for making the papers of the third and fourth Earls of Rosse similarly available. It was pointed out that microfilms of the Herschel papers of the Royal Astronomical Society had been purchased by a number of institutions world-wide, and that historians and astronomers wishing to work on the papers no longer needed to incur the cost of travel to England. By charging for each print a little more than the actual cost, an institution could recover its initial outlay and make its archival holdings available world-wide at no expense to itself.

Other announcements included new's of a catalogue of seventeenth-century books in European astronomical observatories being prepared by Giovanna Grassi; of a new critical edition of the star catalogue in the Almagest by Paul Kunitzsch; and of a forthcoming publication by R. Dvorak to replace the Planetentafeln of P.V. Neugebauer. Ephemerides of the Sun, Moon and planets from -1000 to -600 have been published for the Austrian Academy of Science by H. Hunger and R. Dvorak, though the accuracy at such a distant time interval must be regarded with considerable reserve. Among other topics discussed by the Commission was the possibility raised by Derek Howse of sponsoring at Greenwich in 1984 a symposium to celebrate the centenary of the International Meridian Conference. 
COMMISSION 42: CLOSE BINARY STARS (ÉTOILES DOUBLES SERRÉES)

Report of Meeting, 18 August 1982

PRESIDENT: B. Warner

SECRETARY: E. Budding

\section{Business Meeting I - August 181982}

Minutes of the meeting held in Room $\mathrm{B} 2$ University Complex, Patras from 09.30 until 11.00 .

1. The Chairman (President of the Commission - B. Warner) first listed the proposed agenda on the blackboard and requested comments from the floor. After none were given the proposed agenda was deemed acceptable and adopted. The Commission then elected E. Budding as Secretary for the meetings, whose responsibility was to note the minutes and act as correspondent for the General Assembly Journal "Astrocosmos".

2. The President asked for a moment of silence in memory of five Commission members: A. Beer, G. Chis, K. Serkowsky. M.B. Shapley and J.A.J. Whel an who had died since the 1ast General Assembly.

The President then went on to consider the present membership, currently totalling 211 people, which he noted as a relatively large number. He had received no notifications of intended withdrawals, though some 41 new proposals had come to his attention -7 by direct contact with himself and 34 through the IAU secretariat sent via National Committees. The Fresident urged serious attention to the known activity of proposed Commission Members, mentioning that, for example, merely expressing an interest in the workings of the Commission should not be a basis for acceptance, particularly in view of the large current membership.

On the other hand, he knew of several names who he thought ought to become Commission Members and put some of these before the meeting, at the same time inviting further nominations from those present. The President pointed to the relative shortage of theoreticians among the membership and hoped that a better balance might be achieved. After some discussion of nominations it was announced that a list of those proposed would appear on the Commission's notice board, and the Organizing Committee would in due course make the selection. The President asked all those with information or comments on proposed new members to contact him, or other members of the Organizing Committee, during the week.

3. The President listed the existing Organizing Committee of the Commission (in no particular order) as follows: A.M. Cherepashchuk (USSR), A.H. Batten (Canada), B. Warner (South Africa), R.H. Koch (USA), Y. Kondo (USA), J. Smak (Poland), E.P.J. van den Heuvel (Holland), M. Kitamura (Japan). In a separate group were listed J.A.J. Whelan (GB - deceased), K. Gyldenkerne (Denmark), G. Larsson-Leander (Sweden), M.G. Fracastoro (Italy), S.D. Sinvhal (India) and L.B. Lucy (USA). The last four named had all indicated to the President, either directly or indirectly, a desire to leave the Organizing Committee.

The President then put the following names on the board G. Shaviv (Israe1), K.D. Abyankhar (India), M. Rodonó (Italy), J. Anderson (Denmark), K. Ch. Leung (USA); to stand in place of the members leaving the Committee. These were accepted by the meeting. 
Finally the President proposed that the usual succession to his office be followed, i.e. Vice President A.H. Batten to become the next President. He also proposed that J. Smak become the succeeding Vice-President. These proposals were accepted nem con.

4. The President posed the question as to the real usefulness of the Commission Report. After thanking various Report Contributors he noted that a much more comprehensive record of the work on close binary systems could always be derived from the annual Astronomy and Astrophysics Abstracts where restrictions as to length or content were much less present. He also thought that the General Secretary may be able to offer some advice on the subject. T. Herczeg (supported by $K$. Leung) pointed out a possible usefulness of the Report to members of other Commissions of the Union. F.B. Wood commented on the possible historical value of the Report to future archivists. There was some discussion as to the nature or style of subsections which the President thought could have room for improvement. It was possible that certain lists of references could be more usefully diffused via a centre such as that at Strasbourg.

5. At the last General Assembly Dr. Larsson-Leander had announced his intention to give up the editorship of the "Bibliography and Program Notes" by this meeting. As it was generally agreed that these communications were very useful and should be maintained, a new editorship would therefore be required. The President praised the work of $\mathrm{Dr}$. Larsson-Leander and his team of helpers for their unstinting efforts over so many years, and strong applause greeted the President's request for a vote of thanks. It was pointed out that the IAU does not provide any financial support for the "Program Notes" and finance might become a problem for a new editor. Dr. T. Herczeg was proposed as possible editor to replace Dr. Larsson-Leander, and he announced his willingness to accept the responsibility.

The main uses of the "Program Notes" were thought to lie in their ability to allow members to avoid duplication of effort and offer co-operation on known programmes of work. Dr. Bolton suggested that the notes could contain a questionnaire about current programmes, rather than rely on members writing letters. Yoji Kondo suggested a more basic questionnaire might be issued to determine in what areas the "Program Notes" were felt to be useful. The possibility of a nominal charge, both to help towards costs and to prevent unnecessary postage, was raised, but it was deemed technically difficult and at present unnecessary. The possibility of issue from Strasbourg was again raised in case of financial difficulties. Dr. Herczeg said that he would try to examine the various practical options.

Y. Kondo was invited to speak about setting up a co-ordinated program on Aur stars - to include as a special case the forthcoming eclipse of $\varepsilon$ Aur. He visualised that a great deal of observational material spanning a wide spectral range would be available after the end of the eclipse of $\varepsilon$ Aur in 1985. Also material would be available for $\zeta$ Aur, 31 and $32 \mathrm{Cyg}$, VV Cep and $\mu^{1}$ Sag. $\mu$ Gem was also mentioned as a possibly similar candidate. Members were asked to communicate directly with $R$. Stencel with a view to arranging a Colloquium in 1985 . Asked about the requirement for further organizational support from the Commission, Kondo indicated his desire to spare collective effort. It was thought that he and stencel could effectively deal with the proposal themselves.

The President then spoke on behalf of J. Mattei of the AAVSO to urge IAU support for the observational work of a large number of amateur observers. Some 150 cataclysmic variables have accumulated photometric data on them, and though some of the work prior to $1964 \mathrm{had}$ been reduced and published there is a large backlog of observations requiring effective processing. A figure of some $4.5 \mathrm{million}$ individual observations was mentioned, including a growing quantity of photoelectrically based data. 
Support from the IAU would add prestige to the work; a fuller funding might then be obtained from other sources. The final output was visualised as comprising an Atlas of Light Curves, and magnetic tapes for the corresponding numerical information. A.H. Batten volunteered continued support for this work as incoming President.

The President summarized the discussion in terms of two points: (i) that there was unaninimous support by the Commission for the reduction and publication of these observations as soon as possible, and (ii) that the incoming President would make some private arrangements with Mrs. Mattei to this end. On the basis of (i), the Organizing Committee later submitted the following resolution to the Resolutions Committee of the IAU: "Considering the recent increased interest in cataclysmic variables, and the absence of long term light curves for correlation of observational and theoretical research, we note the data collected by the AAVSO from observers and groups worldwide, and we resolve that these already computerised observations would be a good source of reference and that they should be published". Signed by Janet A. Mattei, President, AAVSO and Brian Warner, President, Commission 42.

8. The President then read out a communication he had received from Dr. Katherine Kron concerning some old observations of the Algol type variables RR TrA, ST Car and $X$ Pic, obtained in 1951-53 by J. Summerville at the request of A. Hog. The data consists of a large quantity of UBV photoelectric photometry, particularly of RR TrA. The problem was - what would become of these observations?

The President suggested that the material be sent to the variable star archives of the RAS, but appealed for any further comments. Chambliss remarked that, if it were possible, the observations could be better served by a more immediate analysis. The suggestion that an announcement in the Inf. BulZ on Variable Stars about these data should be given, was supported by the President.

9. Under the heading of "any other business" the President stated that in view of the number of proposed contributions to the Scientific Meeting to be held on August 23 that the two sessions ( 3 and 4 ) be allowed to run continuously (i.e. without the allocated half-hour break between). About 16 titles had already been received and perhaps more might come. The President thought that the second Business Meeting could also be used for scientific presentations if the demand was sufficient.

In a final point at this Meeting, E. Budding commented on the forthcoming bicentenary of Goodricke's original noticing of the periodicity of Algol and wondered whether some special meeting might be arranged in honour of the occasion. The general feeling appeared to be that the suggestion had come too late for international organization, though no categoric decision was arrived at.

The Meeting was closed at $11^{\mathrm{h}} 00$. 
Report on Scientific Sessions held on 23 and 24 August, 1982

PRESIDENT: Brian Warner

SECRETARY: Edwin Budding

\author{
Abstracts of Papers \\ Optical Spectrophotometry of the HDE226868/Cygnus X-1 System \\ D.R. Gies and C.T. Bolton
}

We have carried out spectrophotometric measures on high signal-to-noise optical spectra obtained by orbital phase averaging lower signal-to-noise high dispersion spectra in ten phase bins. The equivalent widths of the absorption lines confirm the 09.7 Iab spectral classification of the primary. The strengths of all of the absorption lines vary by less than ten percent with orbital phase, except that the He II $\lambda 4542$ line varies by approximately 25 percent with maximum strength coincident with superior conjunction of the supergiant.

Fourier transform analysis of several He I and Si III absorption lines yields $v_{\sin i}=109+2 \mathrm{~km} \mathrm{~s}^{-1}$ and $v_{\text {macro }}=60+10 \mathrm{~km} \mathrm{~s}^{-1}$. If we assume synchronous rotation and a minimum distance of $1.9 \mathrm{kpc}$, then the 1 ight and velocity curves for the system can be combined to yield $30^{\circ} \leqslant i \leqslant 40^{\circ}$ and minimum masses of 24 and 10 solar masses for the supergiant and its companion.

$X$-ray heating of the hemisphere of the primary star that is facing the companion cannot account for the observed behaviour of the He II $\lambda 4686$ emission profiles. However, we have been able to reproduce most of the significant features of the behaviour of these profiles with a model in which the stellar wind from the primary is strongly enhanced near the inner Lagrangian point. At the moment, there does not seem to be any need to invoke a disk around the companion to explain the He II emission line profile.

\title{
$\frac{\text { The X-ray burster } 1735-44: \text { a binary period? }}{\text { J. Hutchings, D. Crampton and A. P. Cowley }}$
}

SIT Vidicon spectra with 30 minute time resolution indicate a 2.86 hour cycle apparently periodic over two nights. Line intensities and velocities of ionized $\mathrm{C}$ and $\mathrm{N}$ vary systematically but in a complex way. Possible binary parameters and mechanisms for exciting the lines are discussed.

The unique binary BE UMa

J. Hutchings, D. Crampton and A. P. Cowley

New radial velocity data refine the orbit and show a 0.15 phase error in earlier ephemerides. Analysis of the light curves considerably constrains model parameters, and suggests that the binary is in an unusual evolutionary state: a very hot subdwarf heating one side of an $M$ giant companion.

The radial-velocity orbit of HD77581 = Vela $X-1$ determined by cross correlation E. J. Zuiderwijk

Radial velocities have been estimated for the early B-type super-giant HD77581 using $6512 \AA / \mathrm{mm}$ Coudé spectrograms obtained between 1973 and 1976 at the European 
Southern Observatory in Chile (ref 1 ). The rectified intensity registrations of individual spectra were cross-correlated with a high signal-to-noise average of all spectra (the template), after sampling in wavelength intervals centred on the strongest spectral features at positions equidistant in $\log \lambda$, and after proper normalisation. The difference in velocity between test spectrum and template follows directly from the position of the maximum of the cross-correlation function. The accuracy of the velocity estimate is between 2 and $3 \mathrm{~km} / \mathrm{s}$ for most of the spectra. A more detailed description of the method and error determination will be given elsewhere.

Orbital elements of the X-ray source Vela X-1 (= 4U 0900-40) are we11 known:$\mathrm{e}=0.089$ and $\omega=168^{\circ}$ (ref 2) - and were used to transform the epoch of observation for each spectrum into true anomaly. The radial velocity variations as a function of true anomaly, however, do not follow the expected sine wave. Instead, during the transition of the $\mathrm{X}$-ray source a disturbance of about $7 \mathrm{~km} / \mathrm{s}$ magnitude appears to be superposed on the underlying sinusoid. The occurrence of this perturbation coincides with an increase in strength of the HeI triplet series (by about $30 \%$ ), and with a distortion in the $H \beta$ profile. Therefore, observations made during $\mathrm{X}$-ray transition were given a low weight in an orbital solution. Then $\mathrm{K}_{\mathrm{opt}} \sim 17 \mathrm{~km} / \mathrm{s}$, as opposed to the $20.5 \mathrm{~km} / \mathrm{s}$ found before (ref 1), and the mass of the $X$-ray source $\mathrm{MX}_{\mathrm{X}} \sin ^{3} \mathrm{i} \sim 1.3 \mathrm{M}_{\odot}$. For an inclination of $75^{\circ}$ (ref 3 ) this yields: $\mathrm{MX}_{\mathrm{X}} \sim 1.45 \mathrm{M}_{\odot}$.

\section{References}

Van Paradijs, J. et al.: 1977, Astron. Astrophys. Supp1. 30, p. 195. Hayakawa, S.: 1982, Space Sci. Rev. 31, p. 283. Avni, Y. and Bahcall, J.N. 1976, Astrophys. J. 197, p. 675.

RY Sct: An International Collaborative Project

$$
\text { R. M. West }
$$

The $11^{\mathrm{d}}$ period close binary system RY Sct is being observed during a concerted campaign. Photometric observations have been obtained in the USSR by Zakirov and Kumsiachvili and analysed by Cherepashchuk; polarimetric observations by Korol (USSR) and Landaberry (Brazil); high dispersion spectroscopy by Sahade (Argentina) and West (ESO); IUE spectra by West (ESO); $21-\mathrm{cm}$ observations have been attempted by Bajaja (Argentina) and Kazès (France), and Belserene (USA) has analysed the historical light curve. Preliminary results indicate that this system, in which the primary star is of spectral-type BO, is surrounded by an expanding gas shell and the presence of gas streams and a rotating torus are indicated. From the evolutionary point of view, RY Sct is probably a younger, hotter and more massive analogue of the $\beta$ Lyrae system. The total mass is in excess of 30 solar masses. The programme continues and a full discussion is expected to become available within the next year or two.

\section{On the Age and Angular Momentum of $W$ Ursae Majoris binaries}

\section{W. van Hamme}

Homogeneous and accurate values of the parameters of $W$ Ursae Majoris contact binaries are required to solve the problems of their structure and age. Photometric elements of many systems are available, but the difficulties of spectroscopic work, because of the rapid rotation of the components, makes reliable determination of absolute dimensions impossible. Nevertheless, the position of 47 W Ursae Majoris systems in a mass-radius diagram was constructed from the photometric elements and only one spectroscopic datum, i.e. the observed spectral type of the binary, and the spectral type-mass relation of Habets and Heintze (1981, Astron. Astrodhys. Supp1. Ser. 46, 193). This position then was compared with the position of the theoretical Main Sequence band, constructed for 11 different chemical compositions from the evolutionary models of Mengel, Sweigart, Demarque and Gross (1979, Astrophys. 
J. Suppl. 40, 733), and differential effects in age were studied. Angular momentummass and angular momentum-mass ratio diagrams also were constructed. The main conclusions are:

(i) the subdivision into $\mathrm{A}-$ and $\mathrm{W}$-type systems does not purely correspond to a separation in age, e.g. some of the A-type systems ( 7 of the total number of 22 of our sample) share the same evolutionary state as the bulk of the W-type systems.

(ii) there is a clear separation in the A-type group between systems which can and which cannot be the evolutionary products of $W$-type systems.

\section{$\frac{\text { A Possible Source for W UMa Systems }}{\text { E. Budding }}$}

A possible source for the W UMa binaries is described, in terms of a Case B type process of mass transfer between components, for which the original mass ratio $\left(m_{2} / m_{1}\right)$ is quite small $(\leqslant 0.2)$. The slow stage of the Case $B$ process is concentrated on and discussed by means of a simplified analytical representation, which reduces, essentially, to a pair of simultaneous ordinary differential equations.

The progress of the resulting common envelope phase is studied by reference to a number of parameters, including some involved in braking mechanisms, which get rid of a certain proportion of orbital angular momentum.

Many observed features of W UMa systems seem able to be broadly accounted for in the scheme, which predicts that a large proportion ( $\sim 10 \%$ ) of seemingly single intermediate mass stars should actually be attended, not too distantly, by low mass companions.

\section{On the Light Curve Analysis of RS CVn Type Binaries Osman Demircan}

The present state of the light curve analysis of the RS CVn tyne binaries has been reviewed. Considerations on the wave-like distortion effect have been critically examined and under the spot model assumption an idea has been introduced to simplify the light curve analysis problem of RS CVn type binaries. The idea is that if spots cover only one part of the hemisphere (say $\Delta \lambda$ in longitude) of a component they ought to be out of sight in a certain time interval. In this interval of time $\left(180^{\circ}-\Delta \lambda\right.$ in phase if Prot $\left.=P_{\text {rev }}\right)$, the light changes should be free from spot effect and can be analysed by considering only the eclipse and proximity effects.

\section{DM Virginis - A Future RS Canum Venaticorum Star? C. R. Chambliss and R. L. Walker}

DM Virginis is a detached eclipsing binary system consisting of two almost identica1 F6-7 IV-V components. Its period is shown to be about 4.6694 days. Light curves in UBV are analysed by the Wood method. Consistent solutions are found, and these indicate that the eclipses are complete and nearly central. The primary is an occultation, and the duration of the complete phase of the eclipses is about 10 minutes. The absolute dimensions of the components are given, and these are shown to be very similar to those of the eclipsing binary $C D$ Tauri and to the subgiant Procyon. DM Virginis is an important system, because its components are essentially free of the close proximity effects found in most eclipsing binary systems. Although this system cannot presently be regarded as an RS CVn system, it is seen as a precursor of this group of stars.

\section{New Results in the Copenhagen Binary Programmes} J. Andersen, B. Nordstrom and J. V. Clausen

The Copenhagen programme (the whole programme is carried out by a group of $\sim 8$ people) of photometric and spectroscopic observations of double-lined eclipsing 
binaries aims at determining masses, radii, and luminosities of sufficient accuracy for critical comparison with stellar structure calculations. During the past couple of years, a large body of new observations has been collected. When these have been fully analysed the existing meagre body of high accuracy data should be at a level permitting a comprehensive discussion of the whole material, planned for 1983 . However, some of the individual unpublished or preliminary results should be of immediate interest to other workers in the field, in particular:

1) Photoelectric photometric and radial velocity observations of Tz For are yielding the first accurate masses and radii for $G$ giants;

2) EM Car will yield one of the very few existing reliable mass and radius determinations for 0 stars (about $20 \mathrm{M}_{\odot}$ );

3) For the we11-known bright system V Pup the interpretation of older radial velocity data as evidence for abundant circumstellar material is found to be incorrect;

4) Several of the newly studied systems have eccentric orbits, and the improved determination of $\mathrm{K}_{2}$, the density concentration coefficient, contribute yet another parameter in the comparison with theories of stellar evolution;

5) Recent photoelectric radial velocity observations of two of the Serpentid group of rapid mass-exchange binaries studied in the satelite UV by Plavec et al. (SX Cas, RX Cas), provide reliable velocity curves for the secondaries which cast doubt on the accepted semi-detached nature of these systems - and thus on the mechanisms for the mass exchange itself.

Spectroscopic Observations of the Eclipsing Triple System $\lambda$ Tauri

Radial velocities from low noise Reticon observations obtained at McDonald observatory of the University of Texas are used to determine the orbital elements of the short-period eclipsing components of this semi-detached system. Combining the minimum masses from the orbital solution with the inclination from the photometric solution, the $B 3 \mathrm{~V}$ star has a mass of $7.2 \mathrm{M}_{\odot}$ and the mid-A subgiant has a mass of $1.9 \mathrm{M}_{\odot}$.

The 33 day periodicity in the velocity residuals, discovered in previous investigations, is confirmed and is present in the secondary velocities as well as those of the primary. The presence of the 33 day periodicity in the velocities of both components means that it can be unambiguously ascribed to orbital motion around a third body. The $K$ and $f(m)$ for the 33 day orbit are $10.1 \mathrm{~km} \mathrm{~s}^{-1}$ and $0.0034 \mathrm{M}_{0}$. From photometric considerations the orbits appear to be nearly coplanar. Therefore, the mass of the third star is $0.7 \mathrm{M}_{\odot}$ and is probably a $\mathrm{K}$ dwarf.

The VW Cephei System

Albert P. Linne11

High speed photometry of VW Cephei discloses important features in the light curves and color curves (Linnell, 1982, Ap. J. Suppl., in press). Light synthesis simulation leads to the following conclusions: (1) None of the proposals in the literature to explain $W$-type $W$ Ursae Majoris light curves satisfactorily represents the VW Cep data. (2) The facing hemispheres of both components must have elevated temperatures, with the secondary slightly hotter. A tentative proposal is that these temperature excesses represent the observational signature of the energy exchange process.

Period changes and the distortion of the light curve of the eclipsing variable CQ Cep with a WR component

J. M. Kreiner and J. Tremko

The variation of the period of the eclipsing variable $C Q$ Cep has been found. The period has been shortening in the time interval from the beginning of the observation until J.D. 2430000 by a value of $0.15 \mathrm{~s}^{-1}$ year ${ }^{-1}$. After J.D. 2430000 the 
period became constant and has been undergoing small variations only. The period changes are ascribed to the non-stationary and non-isotropic mass outflow from the Wolf-Rayet component. The influence of the asymmetry of the light curve on the determination of the epochs of minima is discussed.

\section{The Theoretically expected X-ray Luminosity and the Binary Nature of WR Runaway Stars \\ D. Vanbeveren, W. van Rensbergen and C. de Loore}

The X-ray luminosity expected in WR+ compact star binaries is calculated by combining the production of hard X-rays by accretion and absorption by a stellar wind, in order to decide if the colum density in a WR stellar wind is large enough to absorb all $\mathrm{x}$-radiation.

Stating that there are as many $\mathrm{WR}+$ compact star binaries as $\mathrm{WR}+\mathrm{OB}$ systems depends on the survival probability of the binary during the supernova explosion. This probability is critically investigated and a statistical study is presented based on massive close binary evolution in order to estimate the number of $\mathrm{fR}+\mathrm{com}^{-}$ pact star binaries.

\section{UBV Light Curves of AR Lacertae during 1980-81 and 1981-82 \\ I.-S. Nha, H. S. Park, Y. W. Kang, F. B. Wood, R. M. Williamon and D. B. Leviton}

The UBV observations of the brightest RS CVn-type eclipsing binary star AR Lac were made at four observatories, two in Korea and two in the USA, in 1980-81 and 1981-82 seasons. As a result of the cooperation, two light curves in the yellow and in the blue are completed for each observing season.

The orbital period of AR Lac has decreased by 0.014 since 1977. An analysis of our yellow light curves together with five other yellow curves available in the literature since 1975 shows that there seems no periodicity in the migration of the distortion waves. There is a gradual decrease of at least 0.1 between 1976 and 1982 in the brightness of the cooler component if one assumes that the hotter component is constant.

\section{A Re-analysis of the Eclipsing Binary System VV Orionis}

$$
\text { C. R. Chambliss }
$$

VV Orionis is an eclipsing binary consisting of $\mathrm{Bl} V$ and $\mathrm{B} 4 \mathrm{~V}$ components with a period of 1.4854 days. The eclipses are complete and the primary is a transit. The observations of VV Ori in UBV and in uvby obtained by Chambliss in 1975-79 have been re-analysed with the use of an updated version of the WINK program of Wood. Several solutions were obtained using the normal points (ca. 80 per light curve). These establish fairly reliable values for the linear limb darkening coefficient of VV Ori A $\left(u_{1}\right)$ and approximate values for the luminosity of the third component of this system. Attempts to determine non-linear coefficients of limb darkening for the primary component, however, proved to be unsuccessful. Solutions were also obtained using all observations (ca. 620 per light curve). Very close agreement was found between the values of the geometric elements determined from these solutions and those determined by use of the normal points only. The solutions based on all observations produced reliable values for $u_{1}$, typically about $0.30+0.04$. The results are in good agreement with theoretical limb darkening coefficients derived from model atmosphere calculations. The contribution of VV Ori $\mathrm{C}$ (orbital period = ca. 120 days) to the light of the system was also ascertained, and it was found that this could be best interpreted if this component has a spectral type of $\mathrm{A} 3 \mathrm{~V}$. Mean values for the geometric and photometric orbital elements of Vv ori are also derived. Since VV Ori $A$ is one of the very few early-type stars for which reliable limb darkening coefficients can be empirically determined, it is viewed as being a system of considerable importance. 
On Selected Contact and Semidetached Systems K. C. Leung, L. Z. Shen and D. S. Zhai

Photoelectric observations of AW Lac, BX Peg, BB Peg, and RZ Dra were obtained at Beijing Observatory. The data were analized with the Wilson and Devinney method at the University of Nebraska.

AW Lac : The spectral type of B2: for this system is rather uncertain. An extensive search for photometric solutions at many assumed values of mass ratio, $q$, were made. The most reliable photometric $q$ (i.e. the sums of weighted squares of deviations, $\Sigma$, at the minimum) were found between $0.9<q \leq 1$. The degree of overcontact is large at about $70 \%$. The period and the adopted spectral type combined would imply that this system is located close to the domain of Zero-age Contact systems. Unfortunately, we are unable to determine its absolute dimensions and compare them against the ZAMS mass-radius relation because of the lack of any radial velocity curves.

$\mathrm{BX} \mathrm{Peg}$ and $\mathrm{BB} \mathrm{Peg}$ : These systems have similar spectral types; G5 and F8, and periods; 0.2804 and 0.3615 days respectively. Their light curves are quite similar in shape, with the exception of the significant difference in the heights of the maxima for $B B$ Peg. An extensive search for solutions at the assumed values of $q^{\prime} s$ at $0.35,0.45,0.55,0.7,0.9,1.2$, and 2.8 were made. Coincidentally, both systems are having reliable $q$ values at about 2.8 . The degrees of overcontact are small at $12 \%$ and $10 \%$ respectively. They are both $W$-type systems. It is of interest to point out that while the two systems are similar in most aspects but there is such a large difference in the observed maxima for $B B$ Peg.

RZ Dre : After extensive search for $q$ values, we have improved significantly on the previously reported photometric $q$. The reliable $q$ should be at 0.45 and the system is semidetached with its secondary filling the Roche lobe.

\section{The Period and Light Variations of WW Aurigae}

\section{J. Tremko and G. A. Bakos}

Recent photoelectric observations by Kiyokawa and Kitamura (1975) have shown that the period of WW Aur remained constant for the last 30 years. On the other hand the light curve showed some variations of about 0.02 mag during and outside ec1ipses.

From our photoelectric observations we have derived three new epochs of primary and seven epochs of secondary minima. By means of an $0-C$ diagram we have improved the period to a value $P=2.52501946$ days from the old value, $P=2.52501922$ days. We also found that the secondary eclipse is displaced from the mid-point of two consecutive primary minima by 0.0008 days indicating that an apsidal motion effect may be present.

Variations of the $B-V$ index are obvious at primary minima. The colour index increases by $0.03 \mathrm{mag}$ at mideclipse indicating that the more luminous and hotter component is being eclipsed. A blueing effect at secondary minima could not be observed since no two colour observations were available. Other light variations, possibly due to gas streams have been observed before the first and after the fourth contact. At present time these light changes are being investigated.

\section{Reference}

Kiyokawa, M. and Kitamura, M.: 1975, Annal Tokyo Obs. Series 2, Vol. XV, No. 3. 
This communication summarizes the consequences of a third companion (period $P_{2}$ ) to a close binary (period $P_{1}$ ). Tidal deformation of the close pair is taken into account, and both numerical and analytical methods are used. Apart from the we11-known revolutions of the apsidal and nodal lines, a modulation of the binary eccentricity is important. The amplitude of this modulation grows with the relative inclination between the orbits, and above a "critical inclination" no circular orbits are possible. For point-mass triples, the critical inclination is around $40^{\circ}$, but it increases with the tidal distortion. When the binary apsidal motion alone becomes larger than the three-body contribution, the eccentricity stays nearly constant at all inclinations. Such facts must be included in any study of the orbital evolution of triple systems. The interaction between tidal friction and the three-body perturbations will proceed along qualitatively different tracks in different regions of the $\left(P_{1}-P_{2}\right)-p l a n e$ and for different relative inclinations. A ful1 discussion is very complicated and remains to be done. As for the actual observation of three-body effects, the relevant time-scale is about $P_{2}{ }^{2} / P_{1}$, which is often quite long. An important case is IU Aur, where the eclipse depths are secularly increasing due to a $400 \mathrm{y}$ (approx.) nodal motion. In a few years, the inclination will pass through $90^{\circ}$, and photometric coverage is very desirable.

\section{Close Binary Systems before and after Mass Transfer: Origin of Spectroscopic Semidetached Binaries J. P. de Greve and F. Vansina}

For 26 semidetached binaries, the masses of both components before and after the Roche lobe overflow are determined, assuming a case $B$ of mass transfer. When an amount of mass loss from the system of rough 1 y $40 \%$ is adopted the initial mass ratios are most concentrated in the range 0.35 to 0.75 . Only a few systems have initial mass ratios close to 1 .

\section{SV Centauri - Change of the Light Curve since the Beginning of the Century \\ Z. Kviz}

The comparision of the light curves of SV Centauri for the last 90 years indicates that the minima are getting deeper (especially the primary one) and orobably wider. The primary minimum occasionally shows a flat bottom. Frequent monitoring of the depth and shape of the minima (mainly primary) is urgently needed. 
Report of Meetings

ACTING PRESIDENT (23-26 AUGUST): M. Oda
ACTING PRESIDENT (17-22 AUGUST): Y. Kondo $\quad$ SECRETARY: J. Rahe

In the absence of Commission 44 President R. J. van Duinen, Y. Kondo was designated Acting President until the arrival of Vice-President M. Oda.

\section{BUSINESS MEETING - 18 August 1982}

The first item on the agenda was the election of new officers for the next triennial period and the co-option of new members. M. Oda (Japan), the incumbent Vice-President, was named new President, following the wellestablished protocol, and Y. Kondo (U.S.A.) was elected new Vice-President for the next three-year period. Newly elected members of the Organizing Committee are M. Grewing (F.R.G.), D. C. Morton (Australia), K. A. van der Hucht (The Netherlands) and A. J. Willis (U.K.); the remainder of the Organizing Committee for the next three years are A. A. Boyarchuk (U.S.S.R.), K. Fredga (Sweden), L. Peterson (U.S.A.), E. Peytremann (France), A. B. Underhill (U.S.A.), B. Valnicek (Czechoslovakia) and R. J. van Duinen (The Netherlands).

The Acting President reported with sorrow the tragic death of $D$. W. Schuerman in May 1982.

Commission 44 voted to co-sponsor the proposed IAU Colloquium "The Origin of Non-Radiative Heating/Momentum in Hot Stars", to be held in June 1984; the meeting was proposed by A. B. Underhill.

As no further business meetings were scheduled at the General Assembly, the following actions were taken by polling the members of the Organizing Committee :

Commission 44 agreed to endorse the following resolution. "Commission 44 strongly supports the use of the Space Shuttle science payload for observing Comet Halley during its 1985-86 apparition. Space Shuttle observations can be carried out when the Comet's position is near the sun and other means of observations are not feasible. Such measurements will be a valuable complement to ground-based observations and cometary missions. Multiple launches of the Shuttle cometary science payload will make a significant contribution to the world-wide efforts to study Comet Halley."

Commission 44 also agreed to co-sponsor the proposed IAU Colloquium "Iocal Interstellar Medium", to be held in June 1984 at the University of Wisconsin. The meeting, sponsored by Commission 34, was proposed by Y. Kondo. 


\section{SCIENCE MEETINGS}

Commission 44 sponsored or co-sponsored a number of scientific meetings at the General Assembly, as listed below:

New Ideas About Stars Demanded by Observations from Space, 18 August, chaired by A. B. Underhill, sponsored by Commission 44 .

Galactic Astrophysics \& Gamma-Ray Astronomy, 19 August, G. Morfill, Commissions $44 \& 48$.

UV and X-Ray Observations of Interacting Binary Systems, 20 August, Y. Kondo, Commissions $42 \& 44$. \&44.

Solar Maximum Year, 20 August, E. Tandberg-Hanssen, Commissions 10, 12, 40

Recent Solar Results, 21 August, V. Bumba, Commissions 10, 12 \& 44 .

WG on Objects for the Extragalactic Reference Frame, 21 August, Commissions $7,8,24,33,40$ \& 44 .

Space Telescope, 23 August, F. Macchetto, Commission 44.

Future Space Program, 25 August, M. Oda, Commission 44.

The Interstellar Medium Observed from Space, 25 August, M. Grewing, Commissions $34 \& 44$.

Brief summaries of the meetings have been received from some of the chairmen and are reported in the following. The proceedings of the Joint Session "UV and X-ray Observations of Interacting Binary Systems" is to be published in the Highlights of Astronomy; the summary of this meeting will therefore not be included in the following report.

New Ideas About Stars Demanded by Observations from Space - Anne B. Underhill, Chairman.

The wide topic of New Ideas About Stars Demanded by Observations from Space was reviewed by A. G. Hearn who summarized results concerning the hot stars, by C. Jordan who summarized the results for cool stars, and by C. J. Crannell who summarized the results attained in the domain of high-energy astrophysics.

Hearn noted that the observations (1) that most, if not all, hot stars lose mass at a detectable rate, and (2) that many radiate $X$-rays at a detectable level causing us to modify our concept of what occurs in the outer atmospheres of hot stars. When one is determining the rate of mass loss, it is essential to take account of how the ionization balance changes outward. Radiatively driven instabilities in a wind may be important for generating the very hot gas which is required to radiate $X$-rays at the observed level.

Jordan reviewed the observations of cool stars under three headings: surveys in the lines of $\mathrm{Mg}$ II and in the EUV range; (2) detailed studies of individual stars, and (3) results from $X$-ray surveys. She pointed out that many of the differences noted among the UV spectra from $G$ and $K$ stars appear to be associated with changes in coronal structure and in the presence or 
absence of stellar winds. However, there appears to be no sudden change in total energy input into the chromosphere-corona as one goes along the spectral sequence. A positive correlation between the rate of rotation and the degree of activity in the chromosphere-corona seems to exist for dwarf stars of types $F, G$, and $K$. The activity of stellar magnetic fields appears to be an important factor for controlling what is seen.

Crannell reviewed the instrumentation used to observe stars in the energy range from $10 \mathrm{keV}$ to $1 \mathrm{GeV}$. A wide range of physical processes seems to be active including electron bremsstrahlung, nuclear de-excitation, and particle annhilation. Observations of stars in the high-energy range gives information chiefly about binary stars and neutron stars.

Galactic Astrophysics \& Gamma-Ray Astronomy - G. Morfill, Chairman.

The aim of this meeting was to determine the role of galactic gamma-ray astronomy within the general concept of galactic astrophysics. The timing, at the end of the COS-B mission, was regarded as opportune, because it gives interested astrophysicists the possibility for interdisciplinary studies using the existing gamma-ray data base (e.g., comparison with infra-red, radio, $x$-ray, and other observations), as well as for theoretical studies. The next generation of gamma-ray detectors will probably not be in operation for another 5-10 years, and therefore it is hoped that the proceedings of this meeting can be used (in the intermediate time) as a basis for further studies, as a stimulation for more theoretical work and as an important contribution for defining the aims and operation of future gamma-ray missions. The interrelationship with other branches of astronomy, the astrophysical implications and the study of relevant physical processes using available measurements in the near- Earth environment were important results of the meeting.

The Results of the Solar Maximum Year - E. Tandberg-Hanssen, Chairman.

After an overview of the SMY by de Jager there followed reports on the FBS (Flare Buildup Study), the SERF (Study of Energy Release in Flares), and the STIP (Study of Traveling Interplanetary Perturbations) by Gaizauskas, Rust, and Dryer, respectively. A comprehensive report on the SMY activities in the USSR was given by Mustel, and Wang Shon Gian reported on solar observations in the People's Republic of China. After lunch, highlights from the Hinotori and SMM satellites were discussed by $K$. Tanaka, House and Leibacher. The session concluded with two comprehensive papers on Magnetic Field Studies and Radio Astronomical Studies during SMY by Hagyard and Pick, respectively.

The combined impression made by all these contributions was the wealth of information on solar activity gained during the SMY.

Space Telescope - F. Macchetto, Chairman.

The meetins, which was well attended, was entirely devoted to the Space Telescope project and the expected impact that it will have on astronomy.

Prof. Giacconi discussed the role of the Space Telescope Science Institute in leading the scientific, related activities of the Space Telescope. He 
described its tasks, responsibilities and management organization. He illustrated what an observer had to do to prepare for and to carry out an observing program and what he can expect in terms of support by the Science Institute. He described the selection procedures that the Science Institute will adopt and discussed the policies of data rights and archival regearch.

Dr. F. Macchetto illustrated the preparations that are being made in Europe to fully exploit the Space Telescope data and explained the role of the European coordinating facility as the European Center for Space Telescope Archival Data and Astronomical Software Exchange and Development.

Prof. C. R. O'Dell described the Space Telescope Observatory as a whole, illustrated the present status of development, and showed the excellent quality achieved in polishing the mirrors. He also assured everyone about NASA's commitment to launch the Space Telescope prior to the next IAU General Assembly.

The Principal Investigators for the scientific instruments described briefly their instmuments and the expected scientific performance obtainable from them. The speakers were Prof. J. Westphal (Wide-Field Planetary Camera), Dr. F. Macchetto (Faint Object Camera), Dr. R. Harms (Faint Object Spectrograph), Dr. J. Brandt (High Resolution Spectrograph), Prof. R. Bless (High Speed Photometer), and Dr. R. L. Duncombe (for the Astrometry Team). The session was concluded by an enthusiastic talk by Prof. M. Longair who communicated the scientific excitement of this unique mission to the audience.

In support of this session the Space Telescope Science Institute published a volume which contains the text of each presentation and additional details on the Space Telescope and its complement of scientific instruments. This publication is available from the Science Institute.

The Interstellar Medium Observed from Space - M. Grewing, Chairman.

The existence of a million-degree-component of the interstellar gas is now well established. Dr. G. Garmire discussed the soft X-ray sky maps that have been obtained with the proportional counters aboard the HEAO I spacecraft, and argued that some of the extended features seen in these maps can be accounted for if the solar system is surrounded by a million-degree gas extending out to a distance of about 100 pc. Within this volume must be a number of cool, evaporating clouds. At its edge one finds an expanding shock.

The same local region in space was then discussed by Dr. F. Bruhweiler who reviewed the results from recent UV experiments. It had been known al ready that the H I column density is very low along a few lines of sight. A more systematic study has not shown that low $H$ I column densities are found inthe entire hemisphere between $l=90$ and $l=270$ degrees to diatances of many tens of parsecs. Conversely, looking towards the galactic center, the line of sight seems to pass through a local cloud before entering a region with little $H$. Dr. Bruhweiler went on discussing the implications that the very low $H I$ densities have on the EUV radiation field in interstellar space.

The state of the interstellar gas at the outskirts of our galaxy was considered by Dr. C. Blades who reviewed the recent observations of the galactic halo and halos a round external galaxies. The IUE observations of distant background targets especially provide clear evidence for gas well outside the plane of our galaxy. In addition to the predicted highly ionized species one finds, however, also evidence for neutral or only singly ionized 
constituents, indicating the presence of cool clouds. Indeed, from very high resolution optical observations many of the absorption components are seen to break up into several subcomponents that are extremely narrow.

The contributions that infrared observations can make to extend our understanding of the interstellar medium.were highlighted by Dr. M. Harwit, who chose the Orion complex as an example. The importance of various fine-structure transitions was stressed and the observed strengths were compared to model predictions for $H$ II regions. Turning to very recent results $\mathrm{Dr}$. Harwit reported the detection of the diffuse C II-156 micron emission from the galactic plane that was measured in a very beautiful experiment in which the moon was used as a reference source while it moved across the plane.

Ever since the interstellar reddening law became known from UV wavelengths throughout the optical to near and medium IR wavelengths, has the question been with us what materials are causing the extinction. As Dr. J. Mathis pointed out in the concluding talk of the session, a vast amount of data has been compiled over the past years, mostly from UV observations. He stressed that the features in the extinction curve are still a puzzle. While they can be attributed to a number of materials, all those materials would also produce additional features which are not observed. Dr. Mathis went on discussing what can be concluded from the observed polarization properties, from the peculiar extinction observed, e.g., in dark clouds, and from the correlation with the atomic and molecular hydrogen densities. Some systematic effects are observed in the properties of circumstellar dust depending on the $0 / C-r a t i o$ in the associated stars. Dr. Mathis concluded his review by stressing the need for further IR- and FIR-observations to be combined with observations taken at UV wavelengths. 
Future Space Programmes - M. Oda, Chairman.

Future projects which had been approved or at least authorized in the scientific community were discussed. The Space Telescope and planetary missions were not included, since they were discussed in seperate meetings.

Dr. J. Rosendahl described near term and far term US programmes. They include the Space Telescope and GRO as approved projects, and AXAF whose early start is hoped for. The Explorer project, that is the moderate missions, includes the IR satellite, COBE, EUVE and XTE. The US participation in the ROSAT was decided. As for the Spacelab, several PI class experiments have been approved and infrared astronomy is considered as the next major area to pursue. As for the far term plans the Field's Committee Report was referred to. It was noted that a task force for the space platform or space station had been established.

Dr. Macchetto descirbed the programmes of ESA. The ESA or ESA-related projects COS-B, ISEE, IUE, and GEOS-2 are under operation. Under development are EXOSAT, GIOTTO, Space Tel escope, ISPM and HIPPARCOS. Several projects are under study including DISCO, KEPLER, POLO, ISO, MAGELLAN and X-80.

Dr. Grewing reported the progress of the German X-ray telescope, ROSAT.

Dr. Kardashev reported the USSR programmes for the 1980s. They are: 1) A gamma ray experiment in collaboration with France; 2) $X$-ray observations by the manned Salyut with Czechoslovakia; 3) A future plan for a Salyut $X$-ray telescope with Holland, France, ERG, and ESA; 4) An optical and X-ray telescope with France; 5) A future gamma burst experiments with Veneras and Prognoz stations; 6) A cryogenic submillimeter experiment with France at late 1980s; 7) The "Relict" experiment; 8) RACSAS (Radio Astronomical Cosmical system Aperture Syntheis); and 9) A radio interferometer with a distance of $10^{6} \mathrm{~km}$.

Dr. Y. Tanaka reported the Japanese programes in 1980s which fol low the currently operating HAKUCHO and HINOTORI. They are approved $X$-ray observations ASTRO-B and ASTRO-C plus UVSAT as a planned project.

Dr. W. Cudlip reported the present status of IRAS.

A few reports were contributed including Dr. Samain's UV technology and Dr. Hudson's proposed super-high angular resolution techniques for $X$-ray observations. 
Report of Meetings, 18, 19,20,23,24 and 25 August 1982

\author{
PRESIDENT: A. Slettebak SECRETARY: R. F. Wing
}

I. JOINT DISCUSSIONS

1. Evolution in 01d Stellar Populations in Galaxies (19 August, with Commissions $25,28,29,33,34,35,37$, and 47).

2. The Extragalactic Distance Scale and the Hubble Constant (23 August, with Commissions $25,28,40,47$, and 48).

3. Mass-Loss Phenomena (24 August, with Commissions 29, 35, 36, and 37 ).

\title{
II. JOINT MEETINGS
}

1. Systematic Effects in Abundance Determinations for Metal-Poor Stars (20 August, with Commissions 25, 29, and 37).

This meeting is outined in the Report of Commission 29.

2. Stellar Absolute Energy Distributions (25 August, with Commission 25).

This meeting, which was organized by D. S. Hayes (Comm. 25) and R. F. Wing (Comm. 45), was chaired by Wing. The first session was devoted to absolute calibration techniques in the visual and infrared and to the energy distributions of the Sun and standard stars. The second session dealt with applications to specific photometric systems and comparisons of observed flux distributions to those calculated with model atmospheres. The following papers were presented:

H. Necke1, "The Solar Absolute Energy Distribution".

H. TUg, G. W. Lockwood, and N. M. White, "A Direct Spectrophotometric Comparison, Sun-Vega" (read by Lockwood).

H. Moreno, "Secondary Spectrophotometric Standards for the Southern Hemisphere".

A. Gutierrez-Moreno, "Atmospheric Monochromatic Extinction at CTIO".

D. E. Blackwell, A. D. Petford, and M. J. Selby, "The Absolute Flux of Vega in the Infrared" (read by Petford).

D. S. Hayes, R. F. Wing, S. T. Ridgway, R. R. Joyce, and C. P. Rinsland, "Absolute Calibration of Infrared ( $1-4 \mu \mathrm{m})$ Monochromatic Fluxes" (read by Wing).

E. I. Terez, "Absolute Calibration of the Energy Distribution in the Vega Spectrum over the Region 3110-7500 \&; A. A. Arkharov and E. I. Terez, "The Measurement of the Absolute Spectral Energy Distribution of Vega in the Near-Infrared"; E. I. Terez, "On Stellar Spectrophotometric Standards" (read by Wing). 
E. E. Mendoza, V., "The $\alpha, \Lambda$ Photometric System".

V. Straizys, "A Catalogue of Energy Distribution Curves Representing Different Spectral Types, Luminosities, and Metallictties".

A. G. D. Philip and D. S. Hayes, "Energy Distributions for Horizontal-Branch Stars" (read by Philip).

J. H. Goebe1, "Stellar Infrared Spectrophotometry from the NASA Kuiper Airborne Observatory" (read by C. M. Telesco).

R. F. Wing, C. P. Rinsland, and R. A. Bell, "The Effective Temperature Scale for Late-Type Dwarfs" (read by Wing).

R. Wehrse, "On the Interpretation of Energy Distributions of Spherically Extended M (Super-) Giant Atmospheres".

A. D. Code, "Stars and Galaxies in the Ultraviolet".

\section{MEETINGS OF WORKING GROUPS}

1. WG on Be Stars (23 August, with Commisston 29).

M. Jaschek, Chairperson of the WG, organized and chalred this meeting. During the business portion of the meeting, the following matters were addressed:

(a) The desirability of holding a symposium or Colloquium on the physical parameters of Be stars ( $R$. Viott $i$ ). The consensus was that such a meeting would be premature at this time, in view of the recent IAU Symposium (No. 98) in Apr11, 1981 .

(b) Status report on the Be Newsletter (M. Jaschek). The discussion made it clear that the Newsletter is a great success and should be continued.

(c) New membership in the WG. The consensus is that membership should be open to all who are currently working actively on problems related to Be stars.

(d) Organizing Committee of the WG for 1982-85. An election held at the meeting resulted in the following seven individuals elected to the organizing Committee:

A. Feinstein (Argentina)

P. Harmanec (Czechoslovakia)

L. Houziaux (Belgium)

A. M. Hubert-Delplace (France)
M. Jaschek (France)

A. Slettebak, Chairman (USA)

A. Underhill (USA)

Following the Business Meeting, seven short communications and two invited talks were presented:

J. R. Percy: "Short-Period Photometric Variations in Be Stars".

D. Briot et al.: "Intrinsic Parameters of Be Stars and some Line Continuum Correlations".

Y. Andrillat: "The Infrared Spectra of Be Stars". 

Stars".

G. Peters: "The Nature of the High-Velocity, Discrete Mass Loss in Be

N. M. Ashok, et al.: "Infrared Photometric Studies of Be Stars".

P. Granes and F. Vakill: "Comments on Current Work".

R. Polidan: "A Search for Distant Circumstellar Gas in Be Stars".

A. Underhill and J. C. Mermilliod: Invited talks on "The Evolutionary Status of Be Stars".

\title{
2. WG on Spectroscopic and Photometric Data (18 August).
}

The meeting was organized and chaired by $C$. Jaschek, Chairman of the WG. Following his report on the activities of the WG, there was discussion of future activities and the relationship of the WG to other groups.

The following members of the WG were proposed for 1982-85:
M. Barbier (France)
O. Dluzhnerskaya (USSR)
B. Hauck (Switzerland)
D. Hayes (USA)
N. Houk (USA)
C. Jaschek (France)
M. McCarthy (USA)
J. Mead (USA)

\author{
K. Nandy (UK) \\ L. Pasinetti (Italy) \\ D. Philip, Chafrman (USA) \\ A. Slettebak (USA) \\ F. Spite (France) \\ V. Straizys (USSR) \\ S. Van't Veer (France)
}

3. WG on Ap Stars (18 August, with Commission 29).

The report of this meeting may be found in Commission 29 Report.

4. WG on Standard Stars (25 August, with Commissions 29 and 30).

The report of this meeting may be found in the Commission 29 Report.

IV. MEETING ON "OBJECTIVE-PRISM SURVEYS AND ATLASES" (20 August).

This meeting was organized and chaired by A. Slettebak, at the suggestion of R. West. The latter pointed out that ESO was considering the possibility of making an objective-prism spectral atlas of the southern sky, once the current ESO (R) atlas is finished, and suggested that a discussion of which survey would be most useful would be very desirable. One way of proceeding seemed to be to ask investigators at observatories with objective-prism. facilities to describe their programs and future plans. The following speakers presented brief papers: Telescope".

R. Cannon: "Current and Proposed Objective Prism Work with the UK Schmidt

J. Graham: "Schmidt Programs and Prospects on Cerro Tololo".

N. Houk: "Progress Report on the Revised HD Classification Program". 
A. Savage: "The Optimum Objective Prism Survey for the Identification of Faint Quasars".

M. Schmidt: "Programs and Plans for the Palomar Schmidt Telescope".

A. Slettebak: "Warner and Swasey Observatory Schmidt Programs".

B. Westerlund: "Plans for the Uppsala Southern Station Schmidt Telescope".

D. Crampton: "CFHT Blue Grens Quasar Survey".

H. Maehara: "Kiso Schmidt Telescope Programs".

V. ADMINISTRATIVE MEETING (20 August).

\section{Report of the President}

Since the last General Assembly, Commission 45 co-sponsored the following meetings: IAU Symposium No. 98, "Be Stars", held in Munich, Germany from 6 to 10 April 1981, and IAU Colloquium No. 68, "Astrophysical Parameters for Globular Clusters", held in Schenectady, New York, USA, from 7 to 10 October, 1981.

Elght Circular Letters were sent from the President to the Commission membership during 1980-82, primarily for information purposes and to solicit input for our Commission's activities during the 18th General Assembly.

The Commission 45 Report for 1979-81 was published in the Transactions of the IAU, Vol. 18A. Section reports were written as follows: Classification using Slit Spectroscopy (R. F. Garrison), Objective-Prism Spectral Classification and Classification involving Automatic Methods (D. J. MacConnell), Classification using Multicolor Photometry (V. Straizys), Calibration of Spectral Types in terms of Physical Parameters of the Stars (P. C. Keenan), and Catalogues and Atlases (C. Jaschek).

\section{Membership}

We regret to report that the following members of Commission 45 died since the last General Assembly: M. K. V. Bappu, A. Beer, and H. L. Johnson. Resignations were submitted by $K$. Gyldenkerne and L. N. Mavridis. The following seven new members were proposed and accepted: D. C. Barry (USA), H. H. Guetter (USA), L. Huang (China, Nanjing), T. Lloyd-Evans (South Africa), S. Malaroda (Argentina), E. H. Olsen (Denmark), and H. H. Wu (China, Taipe1). This brings the total Commission membership to 115 .

\section{Election of Officers and Scientific Organizing Committee for 1982-85}

The new officers and Scientific Organizing Committee were elected as follows: V. L. Straizys (USSR), President; R. F. Garrison (Canada), Vice-President; R. A. Bell (USA); M. Golay (Switzerland); A. Heck (Spain); M. Jaschek (France); D. J. MacConnell (USA); A. G. D. Philip (USA); and A. Slettebak (USA).

In some brief remarks, the new President, V. L. Straizys, suggested that the functions of Commission 45 might be extended to include the organization and coordination of research projects. 
4. Reports of Working Groups of the Commission

Brief spoken or written reports of the activities of the Working Groups of Commission 45 were presented by the following Chairpersons:

C. Jaschek: WG on Spectroscopic and Photometric Data.

M. Jaschek: WG on Be Stars.

C. Cowley: WG on Ap Stars.

L. E. Pasinett1: WG on Standard Stars.

\section{Other Reports}

B. Hauck reported on his efforts to implement plate vault data retrieval.

C. Jaschek gave a brief report on the Workshop on UV Stellar Classification which was held at Vilspa, Spain in March 1982.

\section{Proposed Future Symposia and Colloquia}

Commission 45 has agreed to co-sponsor the following Symposia or Colloquia which have either been approved or are under consideration:

IAU Symposium No. 105: "Observational Tests of Stellar Evolution Theory"; Geneva, Switzerland; Sep. 1983.

IAU Colloquium: "Astronomy with Schmidt-Type Telescopes"; Asiago, Italy, Aug. 1983.

IAU Symposium: "Structure and Evolution of the Magellanic Clouds"; Tlbingen, Germany F. R.; Sep. 1983.

\section{International Register of Stellar Spectroscopists (IRSS)}

Th1s publication is edited by $W$. K. Bonsack and A. Slettebak and ma1led to persons listed plus about 230 institutions. In view of support expressed at the meeting and also in the Administrative Meeting of Commission 29, it was decided to continue compiling and distributing this list. 
Report of Meetings held in Patras

PRESIDENT: D. G. Wentzel

SECRETARIES: L. Houzlaux, J. Pasachoff, R. Robbins, V. Ruzd jak

Business Session, 18 August 1982

I. MEMBERSHTP 1982-1985

President: L. Houzlaux

Vice Presidents: M. Rigutt1, C. Iwaniszewska

Organizing Committee: J. Kleczek (Secretary of ISYA)

D. McNally (Editor of Newsletter, ICSU CTS representative)

D. G. Wentzel (Past-president of Commission, publisher of Newsletter)

E. V. Kononovich, M. Gerbaldi, R. R. Robbins (Astr. Education Materials)

S. E. Okoye, A. Sandquist, S. Ferraz-Mello (Visiting Lecturer Program)

Nationa1 Representatives

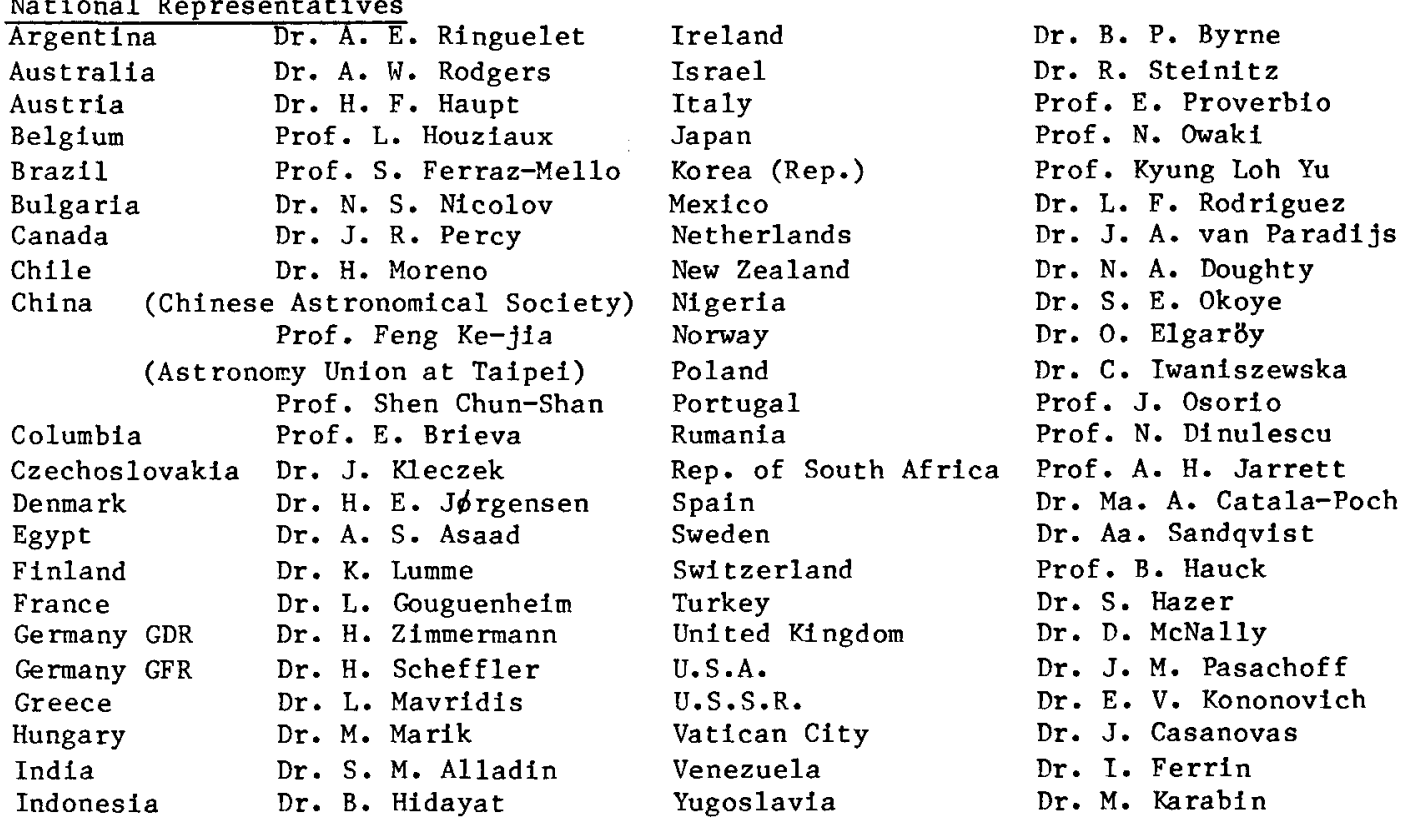

Regular Members. A. Acker, A. Z. Aiad, H. Andrillat, S. M. R. Ansari, I.

Atanasijevic, V. Barocas, L. Bottinelli, W. Buscombe, J. M. Chamberla1n, D. Clarke, S. Codina, E. A. Dibay, F. Dominko, D. L. DuPuy, F. Fuenmayor, H. Gurm, D. B. Hoff, S. Isobe, V. V. Ivanov, J. E. Kennedy, V. Kourganoff, R. C. Madison, J. C. D. Marsh, H. G. Miles, E. A. Müller, B. Onderlicka, W. Osborn, B. Peery, Qu, Q1n Yue, M. RegoFernandez, L. J. Robinson, C. Roslund, A. E. Roy, A. R. Sadik, P. A. H. Seymour, J. L. Safko, T. Schmidt, H. L. Shipman, K. N. Taylor, K. P. Tritton, V. Vujnovic, W. Zealey, M. Zellik. Members not heard from during 1979-82 were omitted from this list. 
Consulting Members. M. L. Aguilar, C. E. Akujor, J. Bishop, M. K. Hemenway, N. T. Jiwaji, P. N. Okeke, L. I. Onuora, K. A. Portzevski1, A. R. Servan, J. Siroky, A. E. Troche-Boggino, R. H. Wilkinson.

Recommendation accepted: The preparation of the membership list before the IAU Gene ral Assemblies will be carried out by the first Vice-president of the Commission. Reason: the correspondence involved provides much useful information for his subsequent term as President of the Commission.

\section{PUBLICATIONS}

The National Reports and Astronomy Education Materials are incorporated in the Newsletters 11-13. The mailing list numbers about 220, with about $20 \%$ each to $\mathrm{N}$. America, Western Europe, Eastern Europe, and roughly 10\% each to Asia, Austra11a, Africa, South America. Additional copies are malled by Houzlaux and McNa11y to local teachers. Surface mail is used.

It was agreed to advertise the Newsletter in the UNESCO Bu1letin and at this IAU General Assembly, but Wentzel stated that advertisement in international journals would yield a mailing list beyond the IAU budget and beyond the capability of the University of Maryland to publish 1t. The IAU budget for Commission 46 publications is Sfr. $5000 \simeq \$ 2500$ for 3 years.

Lengthy discussion centered on the most useful information in Astronomy Education Materials. How important is the attempt to make comments on each published work? Mavridis suggested adding the address of a contact person in each language who might provide more information. For instance, the French and Canadians publish separate lists.

Perkin Elmer's Technical News for September 1982 will have a discussion of astronony and its value, with contributions by C. Townes, J. M. Pasachoff, W. Howard, J. P. Swings and E. M. Burbidge.

\section{COOPERATION WITH INTERNATIONAL TEACHING ORGANISATIONS}

Houziaux mentioned the newsletter of the ICSU Committee on Teaching of

Science. Seven issues have appeared, including some news from the IAU. Since it is difficult to find technicians in many countries, a workshop and newsletter on technicians have been established. A CTS sub-committee has published stx booklets (contact McNally) on learning strategies, including low-cost equipment. A future project may deal with computers and microcomputers.

An international conference on Science, Technology, Education, and the Quality of Life is set for 1985. We should participate to keep astronomy in view of the bodies that make international recommendations. Houzlaux and McNally will work out a proposal. Wentzel has submitted an article to a related publication Sctence, Technology and the Human Prospect.

Houzlaux has not heard from COSTAD since 1978 and did not even receive an answer to his query of their secretariat. Recommendation accepted: that we no longer appoint a delegate.

\section{COMMISSION PROJECTS}

Houziaux will write to the organizers of the IAU Regional Conferences to point out the value of teaching sessions, in accordance with the Commission resolution at Montreal.

Project Contratype has led to the distribution of slide sets and posters to seven countries, supported by the V. M. Slipher Fund of the US National Academy of Sciences, and the circulation of 6 NASA movies to 14 countries. There were no new practical ideas on textbooks. 
V. INTERNATIONAL SCHOOLS FOR YOUNG ASTRONOMERS

This is the most important project of the Commission. The most recent schools were 10-23 September 1979, at La Laguna, Tenerife, Spain (63 students including 12 from America, 8 professors), 17 September - 8 October 1980 at Hvar, Yugoslavia (35 students from Balcan and Mediterranean countries, 8 visiting faculty, stress on practical training, seminars by the participants), and 22 August - 9 September 1981 at Calro, Egypt (28 students, 22 lecturers). The school in Kenya was cancelled for lack of applications, which might be related to the proximity in time and space to the Egypt ISYA, and because no local financial support could be obtalned. The Commission asks ISYA Secretary Kleczek to specia11y inform al1 the National Representatives of countries neighboring to a planned ISYA. Wentzel reported preliminary plans for future ISYA. See below.

A 11st of the approved Rules and Guidelines for ISYA was circulated. It was stressed that each ISYA must be international in faculty and students.

Meetings of the Sub-committee for the ISYA, 19 and 23 August 1982.

The Sub-committee gave preliminary approval to the planning of two ISYA in 1983, after Drs. Hidayat and Fuenmayor provided preliminary budgets and topics. One ISYA is to be in May in Indonesia, with topics on solar physics and galactic structure, in conjunction with the June 1983 solar eclipse. One is to be in October in Venezuela, with some emphasis on space physics. The IAU budget for the ISYA is Sfr. $36000 \cong \$ 18000$ for three years. The two ISYA preliminary budgets each include a $\$ 9000$ contribution from the IAU, but this is not approved until final plans are presented.

Countries mentioned for a possible third ISYA in 1982-1985 are China, Portugal, India and Pakistan. While it seems attractive to involve China fully in IAU activities, the Sub-committee felt that an ISYA in Portugal would be more like1y to come to fruition. Since funding must be sought for any third school, a decision should be made in early 1983.

\section{Session on Visiting Lecturer Program, 19 August 1982}

At a joint session with Commission 38, S. Ferraz-Mello presented the report of the Working Group on the Education of Astronomers from Developing Countries. The following proposal has been formulated and is the basis for a budget recommendation by the IAU Executive Committee for Sfr. $35000 \approx \$ 17000$.

"The International Astronomical Union has decided on a special program to aid countries which wish to inititate or to make substantial improvements in astronomical activities.

The effectiveness of the program requires the collaboration of a University or simflar institution from the country in question, committing itself to participate in a substantial manner and to continue astronomical activities after the completion of this program.

It is not required that the country concerned be a nember of the IAU.

The application should be presented by the country to the Executive Committee of the Union and approved by this Committee.

Costs should be shared fairly, not necessarily equally: in principle, IAU provides the teachers and pays their travel expenses, and the country covers the living costs and the necessary equipment as much as possible. 
A typical arrangement would consist of sending six to elght lecturers to one country in a period of three years, for stays of normally three months each. The three first courses will cover astronomy in general, at the advanced undergraduate level, the following courses can be adapted to the special interests of the host institution."

E. Muller stressed four points made by the Executive Committee: i) the scheme Is not a way to provide paid jobs to young astronomers, 1i) the host country must be not only interested but also committed to pursue education in astronomy after completion of the program, iif) the first project, funded by the IAU and the host country, must be a real success, so that funds may be available from UNESCO for later projects, Iv) the teacher should be fluent in the language spoken by the students. While everyone agreed that a school, for instance, in Peru must have teachers fluent in Spanish, it was also clear that some schools could not possibly be held in the local language, for instance in Indonesia.

Iwaniszewska raised the example of Maria Luisa Agullar, Peru, who wishes to be instructed how to train teachers. Akujor agreed to the need for teacher training. However, this seemed to be a minority view. Both also argued for seeking fellowships to encourage young people to become astronomy teachers, 1.e. fellowships either locally or at established astronomical institutions, perhaps through exchange programs.

other comments were to the effect that lectures should be presented at the appropriate leve1 and there should be practical exercises (Ferraz-Me11o), that a well-documented 1ibrary be available before the lectures start (Moreno), that only experienced teachers be sent, who should keep their home salary while on leave of absence (Hidayat), and that follow-up funds after a project must be sought from UNESCO by the developing country itself (Muller) though perhaps helped by IAU approval (Ferraz-Me110).

In view of reported questions about the propriety of the IAU supporting development of astronomy where there is (almost) none, the Commission passed the following resolution.

Resolution: Whereas astronomy is an integral part of the physical sciences,

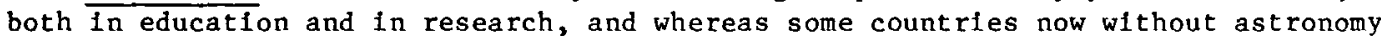
may desire to introduce astronomy in their educational or scientific institutions, be it resolved that the IAU supports the introduction of astronomy in these countries and encourages activities by its Commissions toward this goal.

The following text was approved in principle at the joint Commission meeting, then worked out with the officers of Commission 46 and approved by a later meeting of Commission 38 .

"Commisstons 38 and 46 adopted the report of the joint Working Group on the Education of Astronomers from developing countries. The Working Group, having completed its charge, was formally terminated.

Commisstons 38 and 46 resolved to establish a sub-commlttee to implement the recommendations of the Visiting Lecturers Program in the report of the Working Group and in Agenda item 13(d)2 of the 18th General Assembly of the IAU. Initia11y the membership of the comittee is J. Delhaye (38), S. Ferraz-Me11o (46), D. G. Wentzel (46, Chairman), and F. B. Wood (38). The membership will be subsequent1y expanded to ensure active participation from Developing Countries in its work, with advice and consent from the organizing Committees of Commissions 38 and 46.

The terms of reference for the sub-committee will be: a) to seek individuals and their institutions willing to host a Visiting Lecturers Program; b) to identify 
the local procedures to support the program; in particular, the local body with whom any future contracts should be made; c) to outline a Visiting Lecturers Program agreeable to both host and sub-committee; at this time the sub-committee should identify several possible lecturers with experience in the field of the agreed program, known experience of the developing world and preferably fluency in the local language; (It is recognized that such an identification will not limit final selections.); d) to submit, in 1ight of c) above, a formal proposal to the IAU Executive Committee for a visiting Lecturers Program in a named Developing Country, to be based on a named host institution; e) to submit at the time of d) above, the outline of a second program, such that the General Secretary may seek support through a UNESCO contract; f) and to establish, at the time of d) above, an advisory panel with experience of local conditions, developing countries and the type of program envisaged, in order to advise the Executive Committee on the final selection of lecturers and on the articulation and oversight of the program."

Session on training of teachers, 20 August 1982

Iwaniszewska (Poland) summarized the 35 national reports appearing in Newsletters 11 and 13. Astronomy is a separate course at universities in only 5 countries. In the majority of countries training of teachers is performed at courses given irregularly by university departments $(31 \%)$, summer courses $(26 \%)$ and associations of teachers and astronomers (17\%). Osorio (Portugal) commented that the situation has not improved very much, as only 5 countries have a complete course in ast ronomy.

Hauck (Switzerland) spoke about teacher-training in astronomy in France, Belgium and Switzerland and emphasized the importance of summer schools and 1-2 day courses performed in these countries: He also pointed out difficulties in some countries where there are many educational systems (Switzerland-26 educational systems). Wentzel commented that in the USA there are about 500 educational systems.

Gouguenheim spoke about the summer schools in France. The main goals are to teach the participants in basic astronomy and physics and to perform practical experimental work. She also demonstrated some of the equipment used in the practlcal work.

Owaki informed about the teachers training in Japan. He presented among others an example of a curriculum of Tokyo University for teachers of astronomy and the answers of school teachers to a questionalre regarding astronomy. He stressed that, after graduation, the contacts with teachers are kept in summer schools. Qu spoke about astronomy education in China. Since 1953 about 840 students have graduated at three Universities (in Nanking and Peking). However, it is difficult for graduated astronomers to find a job as there are no courses in astronomy in schools. Moreno stressed the potential of 3 large international telescopes to raise the interest for astronomy in Chile, and he informed about the courses for teachers of mathematics, physics and chemistry in astronomy. Also he pointed out the good interaction with visiting astronomers. Percy described some problems of teaching astronomy in Canada (two official languages, large territory) and presented his plan to publish a "Teachers Guide to Astronomy" which would contain material which the teachers can use directly (maps, tables etc.).

\section{Session on Computers, Television, Activities, 23 August 1982}

Microcomputers in Astronomy Education, D. McNa11y (UK). These are now of sufficiently smal1 cost to give a11 students access to computers and use them as a real tool, not merely for "computing for its own sake". BASIC is the most widespread language, avallable on all machines, with an increasing number of students arriving at universities familiar with it. Graphic capabilities are 
generally avallable and definftely worth having. In five specific areas computers are especially useful:

1) Teaching computing: don't let the computer center do this, because of point (2).

2) Providing sets of programs useful in astronomy: e.g. to compute precession, coordinates, radial velocities, curve fitting, least squares, etc. Eventually students should progress to a better language.

3) Demonstration programs: constellations, bodies moving under central forces, sky to various limiting magnitudes.

4) Difficult concepts: BB radiation, reddening, filters, statistics.

5) Contro1: linking computers, or computers plus instruments, for sophisticated functions e.g. pointing a telescope, guiding a microdensitometer in its measurements.

Programs obtained from outside sources are usually more trouble to adapt than writing your own from scratch. Incompatibilities in the software for various machines are still a major headache. Standards of documentation for interchange of programs between systems are not available.

Radbo (Sweden) has used a computer system for coupled equations, curve of growth abundance analysis for the solar atmosphere, galaxy synthesis. A disk of programs can be borrowed from Bjorn Sundelius (address c/o Mrs. Marie Radbo, Astronomy, Chalmers University, S-41296 Goeteborg).

Television. De Zeeuw (Netherlands) described "Modern Astronomy", a 1981 TV course given in the Netherlands by Leiden Observatory and Teleac, the Dutch TV academy. This course is not part of an open university, because there is no provision for interaction. The program emphasises galaxies, but the 13 lessons of 30 minutes each (20 min of studio time then 10 min interview with an astronomer) are fairly comprehensive of an astronomy syllabus. Animation movies by Seth Szostak are included. 13 radio lessons were a1so produced, and a 450 page book by Nick de Kort, available for $\$ 28$ US. It was a bigger success than expected, w1th 23000 books sold, and will be re-telecast in 1983 with minor changes, even though it was not really for the general public, but for those with some exposure already to astronomy, e.g. amateurs. In producing something like this, close interaction between the TV personnel and the astronomers responsible for content is essential, because some good material looks flat on TV and some bad ideas look good. Control of TV people is essential.

Discussion: Teleac is not very interested in translating this serfes to other languages. There was a British series on commercial TV that stimulated the distribution of 27,000 "starpaks", but the producers there now feel that Carl Sagan has done it all. In the US, it is probably impossible to get veto power over a TV producer. Address: TELEAC, Postbus 2414, 3500 GK Utrecht, The Netherlands.

Radbo: Sweden is an extended country with astronomy at only 4 universities, so an extensive set of correspondence courses have evolved in various fields (with the usual problem of having too little interaction). They added to this concept a series of videocassettes that can be mailed to the home of the student. The cassettes were longer and more detailed than the Netherlands programs, so a book was not as necessary (although a closely coordinated one was produced). A principal aim of the material was the continuing education of teachers. Machine incompatabilities were a big problem. The students for the course were first gathered at Gothenburg for planetarium demonstrations and introductions, then they got the 9 cassettes for 2 weeks at a time (plus a 150-page text). Contacts with the lecturers were maintained by regular correspondence and frequent phone calls, and the lecturers did some visiting. There were essay exams. Production costs were $\$ 60 \mathrm{~K}$, and if the materials can be used for a 10 year period, the cost per course will be $\$ 7 \mathrm{~K}$ (versus $\$ 9 \mathrm{~K}$ per class for conventional techniques). They had 100 students the first time through. Dr. Curt Roslund did most of the manuscript. They have cassettes which can be borrowed for examination. 
Pasachoff (USA) described the use of the Pioneer laserdisk system with 54,000 frames per disk. Quality of visuals is high and either a still or a movie sequence can be searched and projected in five seconds. The system can be interfaced with a computer. A series of 5 disks is being prepared by Center for Aerospace Education, Physics Dept., Drew University, Madison, NJ 07940, USA.

1) Voyager programs (final version price $\$ 320$ for disk)

2) Apo11o/Skylab/Shuttle

3) History of Spaceflight

4) General (all the slides from everyone's catalog)

5) Earth views (Landsat) and planetary geology

The videodisk player is about $\$ 600$, the master disk is virtually indestructible. JPL has produced a Mariner disk (about 50,000 images) which is availab1e now.

Wentzel mentioned that the American Institute of Physics produced and distributes 2-minute TV shows, 5 of them on astronomy. Robbins described StarDate, 2 -minute radio programs produced at the $U$. of Texas, currently reaching about 2 million people per day, on a subscription basis to radio stations. Robbins also commented that 3D terminals now entering the market have tremendous education potential, although they are still quite expensive $(\$ 10,000)$. Nikolov (Bulgaria) reported on a program of 15-20 minute radio lectures that have been produced in his country over the last 4 years. Topics: Introductory material, solar system, stars, extragalactic.

Activities. Ruzdjak (Yugoslavia) described an astronomy activities program in his country, part of a "Science to the Young" movement. Astronomy is one of the competitions in both the 11-14 and 15-18 age groups, even though it is not normally in the school system. Practical work has been encouraged and has grown to $70 \%$ of the projects. Testing did not work out and has been abandoned.

Kleczek (Czechoslovakia) is writing a new edition of Minnaert's book "Practical Work In Elementary Astronomy". Wentzel asked that contributions be sent to Kleczek of new types of experiments, especially in the newer areas of astronomy. Suitable credit can be arranged.

Pasachoff reports some progress toward including astrophysics in the US Graduate Record Examination. Servan (Israel) described the modest growth of astronomoy education in his country. H. Couper (UK) reported on a one-day teach-in at Greenwich. Four astronomers talked to 50 students on what astronomy is real1y $11 \mathrm{ke}$.

Future sessions. Wentzel suggested that the next General Assembly include a session on the use of a planetarium. Should there be an IAU Symposium on teaching? McNally: such a symposium is useful if it concentrates on a particular topic, e.g. teaching of stellar structure. Wentzel: skeptical unless its form is a workshop allowing detalled personal exchanges of ideas. Houziaux: it is more efficient to have teaching sessions at regional IAU conferences.

\section{Session for local teachers, 16 August}

This session, chaired by Prof. C. Antonakopoulos, University of Patras, was dedicated to the teaching of Astronomy at the high-school level. It was attended by representatives from USA, India, Portugal, Taiwan, Greece and several other countries.

The flrst speaker was Prof. L. Mavridis of the University of Thessaloniki who reviewed the educational programs of the Greek University and high school and explained the teaching of Astronomy at both of these levels. 
The next speaker was Mrs. M. Laskaratou, the President of the Hellenic Astronomical Society, who discussed the efforts of the society to popularize Astronomy in Greece and to encourage the teaching of Astronomy in Greek highschools. She reported that the Greek shipowner A. Vergotis has promised to make a large donation for the establishment of an international research center for astronomy and seismology at his beautiful island of Cephalonia.

Prof. Papagiannis outlined the efforts of Boston University to stimulate astronomy education at the high-school level, in part by open houses and the Priscilla and Bart Bok annual awards for projects in Astronomy by high-school students. General discussion included the requests to teach some basic facts such as the cause of eclipses (Bhattacharya, India) and the paralle1 training of astronomers in related flelds (Aller, USA). 
Report of Meetings, 19, 20, 21, 23 and 24 August 1982

PRESIDENT: G. Abe11

SECRETARIES: V. Trimble

I. Novikov

19 and 23 August 1982

JOINT DISCUSSIONS

On 19 August Commission 47 cosponsored Joint Discussion II with Commissions $25,28,29,33,34,35,37$, and 45 on the topic Evolution in old Stellar Populations in Galaxies.

On 23 August Commission 47 cosponsored Jolnt Discussion III with Commissions $25,28,40,45$ and 48 on the toplc The Extragalactic Distance Scale and the Hubble Constant.

The transactions of these Jolnt Discussions are found elsewhere in this volume. Joint Discussion II was under the principal sponsorship of Commission 37 and Joint Discussion III was under the principal sponsorship of Commission 28 .

\section{August 1982}

\section{JOINT COMMISSION MEETINGS}

On 24 August Commission 47 held a joint meeting, with Commission 28, on the topic The Local Supercluster and its Environgent. The meeting, lasting for two sesslons, was cha1red by B.E. Westerlund and G.0. Abe11; the program follows:

R.B. Tully: "Morphology of the Local Supercluster"

J.P. Huchra: "The Dynamics of the Local Supercluster"

E.E. Salpeter: "Dynamical Models of our Virgocentric Deviation From the Hubble Flow"

G.0. Abe11: "Genera1 Superclustering"

J. Einasto, H.G. Corwin, Jr., J. Huchra, R.H. Miller and M. Tarenghi:

"Loca1 Ce11s of the Universe: Two Volds of Diameter $200 \mathrm{Mpc}$

Y. Zeldovich: "Remarks on the Structure of the Universe"

Summaries of these papers are found in the report of Commission 28 .

\section{August 1982}

\section{BUSINESS SESSION}

The President announced, with sorrow, the passing of two members of the Commission since the last General Assembly:

J Neyman

B.M. Tinsley 
The President then summarized other activities of the Commission since the 17th Assembly. These included:

Principal sponsorship (with Commissions 28 and 48) of the I.A.U. Symposium No. 92, "Objects of High Redshift," held at the University of California, Los Angeles, Calffornia, USA, from 28 to 31 August 1979. The Symposium was attended by 146 astronomers from 15 countries. The proceedings have been published by Reldel.

Cosponsorship (with Commisstons 28, 40, and 48) of I.A.U. Symposium No.97, "Extragalactic Radio Sources," held at Albuquerque, New Mexico, from 3 to 7 August 1982. The Proceedings were published by Reldel.

Principal sponsorship (with Commissions 28 and 48 ) of I.A.U. Symposium No. 104, "Early Evolution of the Universe and its Present Structure," to be held at the Orthodox Academy, Kolymbar1, Crete, from 30 August through 2 September 1982, Immedlately following the General Assembly at Patras. (The Symposlum was attended by 191 astronomers from 22 countries; the Proceedings are being edited at this writing, and are expected to be published by Reidel in Spring 1983.)

Election of new officers and organizing commlttee members followed. Those elected, to serve from the end of the General Assembly until the end of the 19 th Assembly in 1.985, are:

President:

Vice President:

Organizing Committee:
J. Audouze

G. Setti

G. Abe11, L.P. Grishchuk, J. Gunn, S. Hayakawa, B. Jones, M. Rees, R. Syunyaev, and H. van der Lann

The following new members were elected to the Commission: D. Basu, G. Chincarini, C.C. Dyer, J. Einasto, H.C. Ford, K. Kellerman, J. Lequeux, T. Lu, F. Melchiorri, W.T. Ni, J.C. Pecker, B.A. Peterson, Q.Y. Qu, H. Saar, V.F. Schwartzman, P.A. Shaver S.F. Shandarin, F.W. Stecker, S. Szalay, V. Trimble, E.L. Wright, and E.J. Zuiderwyk.

H.P. Palmer has resigned.

\section{August 1982}

\section{SCIENTIFIC SESSIONS}

In addition to the two joint discussions and joint commission meeting, the Commisston held two sclentific sessions, both on 21 August. The programs follow:

\section{Recent Observations of Cosmological Interest}

$$
\text { Chair: G.0. Abe11 }
$$

G. Smoot:

P. Kronberg:

M.V. Gorenstein, I.I. Shapiro, N.L. Cohen, E.F. Falco, and A.E.E. Rogers:

"Looking Through a Gravitational Lens: HighSensitivity VLRI Maps of $0957+561$ "

M. Spite and F. Spite: "Lithium Abundance at an Early Age of the Galaxy" 


\section{Recent Observations of Cosmological Interest (Continued)}

M. Longalr :

D.A. Varshalovich:

D. He:
"Evidence for the Cosmologica1 Evolution of the Stellar Components of Radio Galaxies"

"High-Redshift Molecular Clouds and Absorption-Line Spectra of Quasars"

"QSOs in the Direction of the Virgo Cluster"

The Early Unfverse

Cha1r: J. Audouze
Y. Zeldovich:

I. Novikov:

Q.Y. Qu:

D.N. Schramm:

J. Audouze:

J Jones:

B. Jones :
"The Very Ear1y Universe"

"The Origin of the Primordial Perturbations in the Very Early Universe"

"Cosmological Studies in China"

"Planetary-Mass Black Holes Formed During the Quark-Hadron Transition"

"Some Aspects of Primordial Nucleosynthesis and Their Relation to Galactic Chemical Evolution"

"Population III Stars"

"On the Formation of Galaxies"

Full discussions of most of the material covered in the above papers will be published by the time, or shortly after the time, this volume is available; hence, summaries are not given here. On the other hand, a few very general comments on recent advances, especially to supplement or update the President's contribution to Reports on Astronomy, might be in order.

1. The Hubble Constant and Age of the Universe: Aaronson, Huchra and Mould have now observed a number of additional clusters in their ongoing study of the Infrared Eisher-Tully relation and have revised downward somewhat their value for the global Hubble constant -- to about $85 \mathrm{~km}^{*} \mathrm{~s}^{-1} \mathrm{Mpc}^{-1}$. Otherwise, the protagonists in the controversy over the distance scale and age of the universe have not budged from their positions a year ago. On the other hand, as was highlighted in Joint Discussion III, it is becoming increasingly difficult to reconclle what appear to be firm lower limits to the age of the universe from cosmochronology (elther radioactive isotope ratios or globular cluster evolution studies) with a value of the Hubble constant of $\mathrm{H}>75 \mathrm{~km}^{\cdot} \mathrm{s}^{-1} \mathrm{Mpc}^{-1}$, and probably even with $\mathrm{H}>60$. The former point to an age near $16 \times 10^{9} \mathrm{yr}$, and "certainly" not less than 11 or $12 \times 10^{9} \mathrm{yr}$, while if $\mathrm{H}$ is near $100 \mathrm{~km}^{*} \mathrm{~s}^{-1} \mathrm{Mpc}^{-1}$ Friedmann cosmologies with $\Lambda=0$ demand ages less than $10^{10}$ yr, and probably ages for globular clusters less than $8 \times 10^{9} \mathrm{yr}$. 7eldovich reminded the audience at one session at Patras that even in Lemaitre universes with $\Lambda>0$, the "stilistand" 1 n the $\underline{R}(t)$ curve would occur at redshifts of $z \simeq 1 / 2$, inconsistent with observations. This is true, however, for only a density parameter, $\Omega$, near unity, while present observations do not rule out $\Omega=0.04$, in which case a Lemaitre universe with a stationary value of $R(t)$ at a redshift of $z>2$ might still be possible. There is still argument, however, over the possible role of selection effects in the determination of $\mathrm{H}$, and the calibration -- even of the infrared Fisher-Tully relation -- may not be as $f(r m$ as one would hope. For example, very recent (October 1982) work by Sandage on the cephelds in M33 strongly suggests that the modulus of that galaxy should be increased by 0.7 mag. over Baade's value. There is not yet a consensus that the simple Friedmann big bang cosmologies are inconsistent with observations.

2. The Structure of the Universe: Considerable new evidence was presented, both at Patras and at the crete symposium following the General Assembly, concerning the structures of superclusters and volds. On the theoretical side, 


\section{The Early Universe (Continued)}

numerical simulations by Zeldovich and his associates have shown that "pancake"-like superclusters should eventually coalesce, giving the universe a cellular structure, with superclusters, perhaps irregular and filamentary, serving as interstices between the intrasupercluster voids. Observations by Einasto and his coworkers suggest just such a plcture; moreover, Einasto is of the opinion that al1 superclusters are filamentary in structure. Tully's model of the Local Supercluster puts most of the galaxies in a roughly disk-shaped system, but with many in elongated (perhaps filamentary) clouds on elther side. An interesting technique (percolation) for analyzing the surface distribution of galaxies provides a sensitive test of whether that distribution is filamentary or consists of some edge-on sheets of matter, or whether the galaxies clump into randomly shaped configurations. According to the Soviets, the application of the test, at least in some regions, favors the former. In any event, the observations appear to favor flat or filamentary superclusters, and are probably not consistent with the shapes to be expected from gravitational encounters between an initial random distribution of mass points.

3. The Microwave Background: New observatlons, both by the Berkeley and Princeton groups, do not confirm evidence for a quadrupole component to the microwave background radiation, although they do confirm what now appears to be the well-established dipole component. There are also new observations of the spectrum (Berkeley) that cast some question on the reality of the deviations from a black body previously reported, and which have generated so much discussion. There has been some success in interpreting the alleged spectral 1rregularitles in terms of scattering by needle-shaped dust grains produced by "Population III" stars, but perhaps this kind of model will not be required.

4. The Early Universe: The past few years have seen a great flurry of activity in the theoretical study of the early universe. Quantum gravity, grand unffied theories, and super-symmetry theories have been invoked in the hopes of solving a wide range of problems, including those of the baryon asymetry, the monopole, and massive galactic halos. Grand unified theories give mass to the neutrino, and the super-symmetry theories provide massive photinos and massive gravitinos. Particularly interesting is the inflationary universe, first suggested by Guth in 1981. Here the very early universe begins with an exponential expansion similar to the steady-state cosmology. If correct, it could solve the horizon problem (how the universe could ever have been in communication to provide the observed large-scale homogeneity and isotropy) and the flatness problem (how, after expanding in volume by very many orders of magnitude, the universe can have a present density that is within a factor of a few of the critical closure denstty. It is beyond the expertise of this observer to evaluate these new ideas, but clearly they are bringling a great deal of excitement to theoretical cosmology and particle physics.

5. Editorial: The advances in our understanding of the cosmos over the past half-century have been astonishing, but perhaps a bit of caution and humility are in order. It is probably healthy to read a bit of the history of modern astronomy, and learn how quickly Herschel and his contemporaries were to draw firm conclusions about the nature of the nebulae. A theory does not have to be correct because 1 is simple or elegant, and the various protagonists in the controversy over the Hubble constant and age of the universe cannot all be correct. Is it certain that at the XXV General Assembly the Friedmann models of the universe w111 be regarded as firmly established as many seem to regard them today? 
President: F. Pacini

Report of Activities during the XVIIIth General Assembly

Commission 48 was involved in the organization of the following Joint Discussions and specialized meetings:

1. Joint Discussion of Active Galactic Nuclei

2. Galactic Gamma-ray Astronomy

3. Violent Interstellar Medium

4. Impact of Space Research on Astrophysics and Extragalactic Systems, Cosmology

In addition, a Business Meeting was held on August 24, 1982. During the meeting, the proposed composition of the Organizing Committee was approved.

Further activities of Commission 48 around the XVIIIth IAU General Assembly involved the co-sponsorship of IAU Symposium 101: "Supernovae Remnants and their X-ray Emission" (Venice, Italy, August 30-September 2, 1982) and Symposium 104: "Early Evolution of the Universe and its Present Structure" (Crete, Greece, August 30-September 2, 1982). 

TAIRE ET L'HELIOSPHERE)

Report of Meetings on 19 August, 1982

PRESIDENT : H.J. Fahr

Approximately 80 persons attended the meeting in the morning in which 4 review papers and 3 contributed papers were presented concerning the Inner and Outer Heliosphere. After a lively and extended discussion, it was decided to arrange a small workshop on 23 August in order to have a more detailed discussion about problems related to the 3D-structure of the solar wind.

In the afternoon, a meeting was held concerning the heliosphere interface, i.e. the region between supersonic solar wind plasma and unperturbed interstellar plasma. One review paper and three contributed papers were given, during which several aspects of the $3 \mathrm{D}-\mathrm{Deliopause}$ boundary were raised. It appears that the outstanding question is now whether or not there is a supersonic ISM-solar wind interaction. It was decided to further discuss this problem during a forthcoming workshop.

A business meeting with 20 people attending was held in the late afternoon. New members were approved unanimously and Professor S. Grzedzielski (Poland) was unanimously elected new Vice-president of Commission 49. The question of how to further exchange of ideas between Commissions was briefly discussed. 
COMMISSION 50 : PROTECTION OF EXISTING AND POTENTIAL OBSERVATORY SITES (PROTECTION DES SITES D'OBSERVATOIRE EXISTANTS ET POTENTIELS)

Report of Meeting on Site Testing, 19 August 1982

PRESIDENT : F.G. Smith

The morning session was chaired by A. Hoag.

SECRETARY : A.J. Penny

H. Wohl gave the first talk on "Solar site testing in the Canary Isles". He reviewed the history of the JOSO site testing effort since 1967 which began with the consideration of 40 sites, of which 15 were investigated in detail. In 1976 the search was concentrated on Izania on Tenerife and on Roque de Los Muchachos on La Palma. A comparison of the two sites used $40 \mathrm{~cm}$ telescopes looking at the sun for image motion and sharpness amongst other parameters. The Izania site was found to be superior. This was thought to be due to the Caldera on La Palma affecting the seeing more strongly than the topography of Tenerife does for the Izania site. Occasionally. on Tenerife Fried's seeing parameter has been measured as large as $40 \mathrm{~cm}$ which implies that better resolution would be obtained with telescopes larger than those used in the site testing. In a comment from the audience, the importance of such a large value or $r_{0}$ for active image compensating work was pointed out.

A. Penny then gave P. Murdin's report on "Protection and Investigation of the Roque de Los Muchachos site". Microthermal sensors at heights of $3 \mathrm{~m}$ and $12 \mathrm{~m}$ were used simultaneously for eight nights at two positions within the site. One was at Fuente Nova at the edge of the caldera where the original testing had been done and the other was $250 \mathrm{~m} \mathrm{~N}$ and down hill from these, at a proposed site for the $4.2 \mathrm{~m}$ telescope. When the wind is southerly, flowing over the rim of the caldera, turbulence occurs at both 3 and $12 \mathrm{~m}$ heights at the rim, but only at the $3 \mathrm{~m}$ height at the down hill site. The suitability of the proposed $4.2 \mathrm{~m}$ site was thus confirmed. It is suggested that the JOSO comparison of the Roque and of Izania was affected by local conditions very close to the caldera rim. Further microthermal tests will be carried out to investigate local effects and to provide a comparison with telescope seeing measures.

M. Walker reported on "International Comparisons of Seeing". He reviewed the Polaris trail method which has new been used to test fifteen sites and has confirmed the pattern that isolated conical peaks in the five regions of the lower-middle latitude band have the best seeing. A recent investigation of the NW Cone (a site proposed for the California 10m telescope) on Mauna Kea and of a site off the Mauna Kea summit on the north shield was described. At the shield site microthermal activity was found and also the seeing was worse than on the NW Cone.

D. Crawford reviewed the Dark Sky Survey. A project has been funded to collect data from existing sites, to extend the Polaris trail method, and to measure sky brightnesses and night time water vapour leve1s. Seven night time water vapour meters have been built and distributed. A comparison between Mauna Kea and Mt Graham, Arizona, is being undertaken and further site testing is planned with the NTT $15 \mathrm{~m}$ telescope in mind.

G. Lockwood spoke on "Experiences with long-term extinction measurements". Tests at Tucson and Flagstaff have found no effects from man-made pollution. Volcanic activity however has sometimes dramatic effects on aerosol extinction, and there was also evidence for climatic effects. In the south-western us, extinction is higher in summer than in winter. There appeared to be no change in the ozone extinction with the solar cycle. 
J. Tremko reported on "Long-term variations of atmospheric transparency from measurements at Skalnate Pleso and Brno". From observations over 20 years, totalling 400 nights at Skalnate Pleso, long-term variations in the aerosol extinction were found, possibly correlating with the solar cycle. The Brno observations showed that in an urban environment the wavelength power law exponent of the aerosol extinction varies between 1.3 and 3.6 .

A. Hoag reported on "Sky Brightness monitoring". Observations over the sky at a site and zenith measures at locations around the site can be used to estimate local and non-local contributions to the sky brightness. Work at Lowel1 and Kitt Peak was discussed and the effect of night sky emission lines and the solar cycle mentioned. Following this, M. Walker mentioned he had been monitoring the sky brightness at an isolated site in California and found a half magnitude increase in brightness from 1976 to 1980, which result matched the experience of the UK Schmidt in Australia.

$\mathrm{J}$. Bhattacharyya reported on work in Bangalore by $\mathrm{S}$. Venkatesh on the correlation between seeing as measured by a $1 \mathrm{~m}$ telescope and microthermal activity. Cross-spectral analysis of the seeing with the microthermal activity and wind speed showed characteristic peaks at preferred frequencies with high coherence.

D. Carter described the New Zealand six site extinction monitoring program and C. Balnco described the Italian past and present site analysis program.

The afternoon session was chaired by F.G. Smith.

G. Alvarez reported on "Protection of the IAC Observatories in the Canary Isles". The aim is to make the Observatorio de Roque de Los Muchachos site an astronomical reserve. Already air routes above the site have been derouted and the installation of radar stations avoided. The implementation of Commission 50 recommendations is being discussed with the local government and the Spanish government is studying giving the IAC executive faculties concerning the protection of astronomical facilities. A sky brightness investigation, monitoring and control project has started. On Tenerife, the presence of artificial light is clear, but on La Palma there is no problem at present.

A. Asaad spoke on "Sky Brightness at Kottamia". Kottamia is suffering from light pollution caused by the growth of Cairo and looking to Cairo at a zenith distance of $45^{\circ}$, a sky brightness increase of $30 \%$ is present. The projected growth of Cairo and the establishment of new towns will seriously affect the site. Options open to deal with this are to reduce the effect by light pollution control laws or by moving to a high site in Sinai.

D. Crawford spoke on "Tucson experience of light pollution ordinances". Two years ago stricter ordinances were adopted by the city and county and there has been good press coverage and good progress in light pollution control. The counties surrounding Mt Graham and the MMT are being lobbied and one man was working half-time on such lobbying. The time control of outdoor lighting was being pressed. A major argument which can be used is financial saving, and there is good cooperation with the professional lighting engineers. Much can be achieved by determined efforts.

P. Burke also spoke on the "Solar Power Systems". He described the characteristics of the proposed systems and the NAS technical report on it. The deleterious effects on optical, infrared and radio astronomy and on communication with deep space probes were described. There are international treaties preserving wavebands for radio astronomy but none for optical. Whilst NASA no longer have it as an active project, there is the longer term possibility that conditions might change to resurrect it. If this does happen it will probably be as an international large scale project. Astronomers will not have great political strength to oppose it, but can ensure that any project is thoroughly and properly analysed before being started. 
P. Boyce also spoke on the Solar Power Systems. He reviewed the various ways by each its effect on astronomy could be greater than at first envisaged and pointed out that by the time any possible system is in operation the growth in ground based astronomy will mean there will be even more facilities affected. He reiterated the importance of educating governments and peoples of the value of astronomy.

C. Bolton said that the Canadian Astronomical Society and Canadian IAU National Committee wish to have a watching brief on the subject. He mentioned some of the technical problems not so far studied such as that of the rocket launches and of the scattering of light by the occasional volcanic dust layers.

F.G. Smith explained that this was the first time the IAU had discussed the problem and represented the start of IAU interest in the subject. The example of the work of Commission 50 on the protection of sites from ground based effects which had been effective by first studying the technical aspects and then drawing up recommendations pointed a way forward. The effects of all present and future space work should be investigated.

A draft resolution for submission to the General Assembly stating : "Commission 50,

considering with deep concern the proposals for a Satellite Power System which would place very large structures into Earth orbit with the potential of destroying the conditions for a major part of astronomical research in many regions of the electromagnetic spectrum,

recommends national representatives to bring this concern to the notice of space agencies in their countries, and to ensure that the IAU is kept fully informed of proposed developments in space techniques which would involve dangers to astronomy."

was adopted unanimously. F.G. Smith said that the president of Commission 50 would be dealing with the administrative matters concerning this.

The meeting then turned to business matters. F.G. Smith announced his recommendations to the IAU of A. Hoag as president of this Commission and of S. van den Bergh as vice-president. He described the report of the Commission and on the Commission membership. A Working Group on the monitoring of extinction was set up with P. Edwards as chairman, who should be contacted by those wanting to join. A. Hoag mentioned the possibility of a Working Group for guidelines on site evaluation. 
COMMISSION 51: SEARCH FOR EXTRATERRESTRIAL LIFE (RECHERCHE DE LA VIE DANS L'UNIVERS)

\section{PRESIDENT: M.D. PAPAGIANNIS}

VICE PRESIDENTS: F.D. DRAKE and N.S. KARDASHEV

ORGANIZING COMMITTEE: R.D. BROWN, P. CONNES, G.D. GATEWOOD, L. GOLDBERG, J. JUGAKU, G. MARX, F. PACINI, M.J. REES, V.S. TROITSKY

\section{INTRODUCTION}

The possibility that life might exist in many other places in the Universe has puzzled the minds of scientists and laymen alike for thousands of years. Nearly 2,400 years ago in his book On Nature the Greek philosopher Metrodorus of Chios (as best as $I$ can translate $i t$ ) was writing: "It goes against Nature, in a large field only one shaft of wheat to grow, and in an infinite Universe to have only one living world." At the end of the $17 \mathrm{th}$ century the famous Dutch physicist and astronomer Christian Huygens wrote the book Cosmotheoros in which he tried to show with scientific reasoning that the other planets must also harbour life. He also tried to reconcile sclence and theology arguing that uninhabited worlds are a great waste, which is uncharacteristic of God who has a purpose for everything. More recently, in the middle of the 19 th century, the great German mathematician Karl Friedrich Gauss proposed to plant a colossal forest in the shape of an orthogonal triangle, to signal to astronomers of other worlds that the Earth is inhabited by intelligent beings who are well acquainted with the Pythagorean theorem.

For thousands of years all we could do about this intriguing subject was talk and philosophize. In our days, however, the situation has changed drasticaliy. Now, our radio telescopes can receive radio messages transmitted by similar instruments thousands of 11 ght years away, and have already tried repeatedly to do that. Our spaceships are able to explore every part of our solar system, and have already landed on Mars to analyze its soll for signs of biological activity. The electronic and computer support of our optical and radio telescopes is improving at a $f$ ast pace, and in a few years, around 1985, we will have our first large telescope in space.

The first radio search was carried out in 1960 by Frank Drake, the Vice President of our Commission, who in his project OZMA looked for radio messages at the $21 \mathrm{~cm}$ line of hydrogen from two neighboring stars, epsilon Eridani and tau Ceti. Up to now, there have been about 35 radio and optical searches, which for the most part were individual efforts without any overall coordination or long term planning. They were carried out by a few brave astronomers who usually had to work without research grants and who often had to face the skepticism of even some of their own colleagues. In the $70^{\prime} \mathrm{s}$, however, the situation began to change with an accelerating pace.

\section{DEVELOPMENTS IN THE LAST 12 YEARS}

The Byurakan conference in the Soviet Union in 1971 on CETI (Communication with Extra-Terrestrial Intelligence), which was co-sponsored by the USSR Academy of Sciences and the USA National Academy of Sciences, was an important landmark in the establishment of this new field. Credit must be given to the organizers of this meeting: the Soviet astronomers, V. Ambartsumian, N. Kardashev, I. Shklovsky 
and V. Troitsky, the American astronomers, F. Drake, P. Morisson and C. Sagan, who also edited the proceedings published by the MIT Press, and to R. Pesek of Czechoslovakia, who was also representing the International Astronautical Federation. Another important factor in gaining acceptance and recognition for this new field was the stubborn persistence of NASA in the search for extraterrestrial life which, in spite of repeated rejections by the U.S. Congress, was kept alive thanks to the dedicated leadership of a few NASA officials, such as G. Soffen and J. Billingham, and thanks to the tireless efforts of a few other scientists, such as B. Oliver.

A big step forward was also the Joint Session on "Strategies for the Search for Life in the Universe" which was held during the 1979 General Assembly of the IAU in Montreal. It was organized by an international committee chaired by $M$. Papagiannis, and was co-sponsored by three IAU Commissions: 16. Physics of P1anets, 40. Radio Astronomy and 44. Space Astronomy. It represented the first time that the IAU was dealing with this subject. The whole-day session generated great interest and was attended by more than 300 astronomers. The following night, when in an open session chaired by Leo Goldberg, F. Drake and M. Papagiannis summarized the presentations and conclusions of the joint session for the general membership of the IAU, the large auditorium of the University of Montreal was packed with more than 1000 people. The success of these meetings in Montreal, showed the great interest that exists within the astronomical community for this new fleld and led to the formation of our new Commission at the next IAU General Assembly in 1982.

In the three year period between 1979 and 1982 , several other events occurred which strengthened even further the case for the new Commission. The proceedings of the Montreal joint session, edited by M.D. Papagiannis, were published in 1980 by Reidel under the title Strategies for the Search for Life in the Universe. The MIT Press published in 1981 the volume Life in the Universe, which was edited by J. Billingham and contained the studies of several NASA workshops and the proceedings of a conference organized by NASA at the Ames Research Center 1n 1979. The book Extraterrestrials - Where are They?, edited by M. Hart and B. Zuckerman, was published by Pergamon Press in 1982 and contained the proceedings of a conference held in the fall of 1979 at the University of Maryland, USA. Another international conference on SETI, a follow-up of the Byurakan conference, was held in Tallinn, USSR in December of 1981. It was sponsored again by the USSR Academy of Sclences, which maintains an official SETI section, and was chaired by V.S. Troitsky. Its proceedings will be published soon.

A very significant development in this field was the publication by the U.S. Academy of Sciences in 1981 of the report: Astronomy and Astrophysics in the 80's which was prepared by a blue ribbon committee appointed by the Academy and chalred by G. Field. This report endorsed the establishment of an active program for the Search for Extraterrestrial Intelligence (SETI) and recommended to the Government the allocation of 20 million dollars for this program during the decade of the $80^{\prime} s$.

All these developments came to a climax at Patras, Greece in August 1982 when the General Assembly approved the establishment of a new IAU Commission on this subject. A great pay-off to all these efforts came soon after. In October 1982, the U.S. Congress passed NASA's budget including, for the first time, a budget line for SETI. With the establishment of the IAU Commission and with government support for work in this field, the search for extraterrestrial life had finally come of: age.

\section{THE ESTABLISHMENT OF THE NEW COMMISSION}

Following the success of the Montreal meetings in 1979, several people wrote to P. Wayman and to M. Papagiannis inquiring if the IAU could become more active in this field. It is to the great credit of the Secretary General of the IAU that he took this subject under his wing, corresponded with many people, talked to other international unions, and finally brought it to the Executive Commlttee, which made the courageous decision that the IAU ought to assume the leadership in this field 
and approved the establishment of a new IAU Commission on this subject.

M. Papaglannis kept corresponding with $P$. Wayman throughout these three years, and at his request proposed the names for a 12-member advisory board, which were approved by the E.C. Through further consultations we settled on the name and on the objectives of the new Commission. On August 19, 1982, the advisory board met at Patras with P. Wayman who told us that the Executive Committee had approved the establishment of the new Commission and urged us to proceed with organizational matters.

The news about the new Commission was carried by the ASTrocosmos, the newspaper of the General Assembly, and a few announcements were posted in the halls of the University of Patras. The response was amazing as more than 100 IAU members asked immediately to join the new Commission. More members were added in the ensuing months through correspondence or personal contact. The Commission now has a membership of close to 200, about 180 IAU members and 20 consultants. The 1 atter are distinguished scientists who share many common interests with the new Commission, but whose work is primarily in other disclplines such as Physics (E.M. Purcell, a Nobel laureate, USA), Life Sciences (G. Soffen, Director NASA, USA), Chemical Evolution and the Origin of Life (C. Ponnamperuma, USA), Astronautics, (R. Pesek, Czechoslovakia), Geophysics (B. Murray, USA), and others. The variety of fields represented simply conflrms the fact that the search for extraterrestrial ife is a subject of broad appeal that touches many disciplines.

It was decided that the name of the new Commission will be: IAU Commission 51 - Search for Extraterrestrial Iife. Michael D. Papagiannis of Boston University was elected the first President of the new Commission, which was allowed to have two Vice Presidents, Nikolai S. Kardashev of the Center for Space Research of the USSR Academy of Sciences and Frank D. Drake of Cornell University. The other nine members of the inftial advisory board became the Organizing Committee: Ronald D. Brown (Australla), Plerre Connes (France), George D. Gatewood (USA), Leo Goldberg (USA), Jun Jugaku (Japan), Gyorgy Marx (Hungary), Franco Pacini (Italy), Martin J. Rees (UK) and Vasevolod S. Troitsky (USSR). The IAU General Assembly at its second session on August 26, 1982 approved unanimously the establishment of the new Commission and the names of 1 ts officers.

\section{OBJECTIVES AND FUTURE PLANS}

The areas of interest of the new Commission include the following:

1. The search for planets in other solar systems.

2. The evolution of planets and their ability to sustain life.

3. The search for radio signals, intentional or unintentional, of extraterrestrial origin.

4. The search for, and study of, biologically relevant interstellar molecules.

5. The spectroscopic detection of blological activity from primitive forms of 1 ife.

6. The search for different manifestations of advanced civilizations.

7. The coordination and promotion of these activities at the international level, and the collaboration with other international societies (biological, astronaut1cal, etc.) that have related interests.

In this last area we have already made substantial progress. M. Papagiannis was appointed the official representative of the IAU to the IAF (International Astronautical Federation) and as such attended its 33rd Congress in Paris at the end of September 1982. The IAF maintains an active SETI Committee, of which he was elected a member. In reciprocity, $R$. Pesek and J. Billingham, the Chairman and co-Chalrman of this SETI Committee, were elected consultants of our new Commission, thus ensuring excellent communications between these two international bodies. Good contacts also have been established with ISSOL (International Society for the Study of the Origin of Life). Its Vice President, C. Ponnamperuma, has been elected consultant to out Commission and $M$. Papagiannis, who is already a member of ISSOL, will be proposed to become a member of its international advisory committee. We are continuing the dialogue with IUBS (International Union of Bio- 
logical Sciences) which was initiated by P. Wayman, and we expect we will also be talking with COSPAR.

We are already working on several activities, including efforts:

1. To computerize our membership list and distribute it to all our members and consultants to promote closer contacts among all of them.

2. To publish a Newsletter about 3 times a year which will be mailed to all of our members and consultants. It is expected that they in turn will be submitting to the Newsletter brief news releases on thelr recent work which will speed up communications among the people working in this fleld. NATURE has also requested to recelve copies of this Newsletter to communicate all new developments in this field to its wide readership.

3. To hold its first scientific meeting, hopefully an IAU Symposium, in the summer or fall of 1984. Our first choice for this event is Hungary which is centrally located and easily accessible from many parts of the world thus assuring a greater participation. At this first meeting, in addition to the normal scientific program, we also hope to have workshops which will try to plan and coordinate activities and research programs in our field around the world.

\section{RECENT RESEARCH WORK}

According to a sumary compiled in 1981 by $\mathrm{J}$. Tarter of NASA-Ames, and some additional 1982 information from colleagues, in the 22 year history of radio searches from 1960 (project OZMA) to the summer of 1982, there have been at least 31 independent radio searches. These involved at least 12 different radio observatories in seven different countries (USA, USSR, Australia, Canada, France, W. Germany, Netherlands, and Japan). They have compounded about 5,000 hrs of observations, covering a variety of targets, 'frequencies, bandwidths, and resolutions. In the $60^{\prime}$ s we only had 4 radio searches, but in the $70^{\prime}$ 's we had 21 , and in the $80^{\prime} s$ we already had 6 .

These six most recent ones were: A search along the galactic rotation axis by Lord and $O^{\prime}$ Dea at the $2.6 \mathrm{~mm}$ line of $\mathrm{CO}$ with the millimeter radio telescope of the $\mathrm{U}$. of Massachusetts. Two searches at the hydrogen 1ine by Israel and Tarter ( 85 star fields) and by Shostak and Tarter (galactic center) using the Westerbork array in the Netherlands. The search at the hydrogen line by Tarter, Duquet, Clark and Lesyna of 210 solar-type stars with the Arecibo radio telescope. The observations of Biraud and Tarter at the $\mathrm{OH}$ line of 102 stars with the Nancay, France decimetric radio telescope. And finally, the ultra-high resolution observations by Horowitz of 250 solar-type stars at the hydrogen line and at twice the hydrogen line frequency with the Arecibo radio telescope.

Several other radio searches are also now being instigated. The Russians are building a network of 100 SETI dishes to obtain an all-sky coverage for broad band pulses. The Japanese are planning searches at the ant1-maser line of formaldehyde at $4.83 \mathrm{GHz}$ with the Nobeyama millimeter radio telescope, and Harvard University in the USA is converting its $26-\mathrm{m}$ radio telescope to a SETI dedicated facility. This project is being supported by private funds from the Planetary Soclety and will soon be conducting "meridian transit" searches in the $-30^{\circ}$ to $+60^{\circ}$ declination range. The project is estimated to last 5 years with searches made at several "magic" frequencies ( $\mathrm{H}$, OH, etc.) using the "Suitcase SETI" spectrum analyzer deve1oped by Paul Horowitz of Harvard and colleagues from Stanford University. This analyzer has a frequency resolution of $0.015 \mathrm{~Hz}$ over a bandwidth of about $1 \mathrm{kHz}$ and is very effective in eliminating terrestrial interference. It presupposes, however, that the signals are transmitted exactly at the line frequency, having already been corrected by the transmitting civilization for all Doppler effects relatively to our Sun. When this equipment was used with the Arecibo radio telescope in May 1982, it achieved a sensitivity of $2 \times 10^{-27} \mathrm{~W} / \mathrm{m}^{2}$ in one minute, which means that it could have detected a radio signal of only $1 \mathrm{~kW}$ power transmitted by an Arecibo-like antenna about 100 light years away.

Besides the radio searches, in the past 20 years we also had 3 optical searches. 
A search by Wishnia with the Copernicus satellite for UV laser lines from 3 stars, a search by Schwartzman in the USSR for short optical pulses from peculiar objects, and an optical search by Freitas and Valdes in the United States for objects in the L4 and L5 regions of the Earth-moon system.

\section{IMPORTANT TECHNOLOGICAL DEVELOPMENTS}

Meanwhile great progress is being made in the development of a megachannel spectrum analyzer for our radio searches. We now have (NRAO, JPL, Harvard) several 65,500 channel analyzers, but a group at Stanford University. under the direction of Allen Peterson, is now building a revolutionary 8 million channel analyzer. The design, employing a novel approach to digital spectrum analyzers, has been completed and a first generation model with 72,000 channels is now ready for testing. This analyzer is being bullt in modular form which provides great flexibility and allows for continuous expansion. Its capacity will continue to grow in the coming years and is expected to reach its full strength of $8 \mathrm{million}$ channels around 1987 . It will have a bandwidth of $8 \mathrm{MHz}$ and will be able to simultaneously analyze the input signals into several frequency resolutions ranging from $1 \mathrm{~Hz}$ to $1 \mathrm{kHz}$. A spectrum analyzer of this capacity and with these special features will accelerate immensely all radio searches that try to examine a large number of stars over a considerable frequency range, such as the "water hole" between the $H$ and OH lines. It also would be ideal for radio searches for unintentional radio transmissions (such as our own TV and radar signals) from several nearby stars, which must span a wide frequency range with a high frequency resolution. The completion of this megachannel spectrum analyzer will be a major technological breakthrough for our radio searches.

Another area where great progress is being made, is the development of new instrumentation for the astrometric detection of planets in other solar systems. Up to now, the three observatories in the United States working on this problem (Sproul, Allegheny, and Naval), were making their astrometric observations using photographic methods. Two new techniques, however, are now being developed with great potential for the future. The one is an interferometric system that is being built by Shao of NRL and Staelin of MIT, and the other is a photoelectric system which is being developed by Gatewood and his colleagues at the Allegheny Observatory. A prototype of the interferometer was tested in 1980 tracking the position of Polaris, while a more advanced version capable of determining the angular distances between stars is now being tested at the Mt. Wilson Observatory. This technique, by measuring separately the positions of the red and blue fringes every few milliseconds, is capable of removing much of the effects of the atmospheric seeing and is expected ultimately to reach precisions of about 0.1 milliarcseconds (mas).

A prototype of the Multichannel Astrometric Photometer (MAP), as the Allegheny group calls their device, was also first tested in 1980, but a later version of the MAP is now ready to begin surveying activity at the Allegheny observatory. The projected precision is 2-3 mas per hour, which surpasses the precision obtained with photographic methods over an entire year. The precision is now actually 1imited by the quality of the 30-inch objective lens of their refractor telescope, but a new lens figured for red light is now on order, largely thanks to the generosity of Bernard Oliver of the Hewlett-Packard Co., and is expected to become operational in 1984. This w111 further improve the precision of the system and will allow an intensive search to begin for Jupiter-like planets in any of the nearest 100 to 200 stars.

The accuracy of astrometric observations from the ground with the MAP is limited by atmospheric seeing to a precision of about 1 mas. The precision of the system, however, can be improved by about 3 orders of magnitude to about a microarcsecond when used above the atmosphere with a space telescope. A precision of 1 Has will finally allow the detection of Earth-1ike planets in other solar systems. The basic problem with space observations is the need for a good reference system. 
Lockheed, however, which conducted a feasibility study of these new concepts, concluded that Earth-like planets can be detected with present technology using a double aperture, double MAP configuration, that would measure sufficient reference stars to obtain microarcsecond precision in less than 10 hours of space observations. It appears that we can expect great developments in the search for planets in other solar systems during the $80^{\prime} \mathrm{s}$, especially when the new techniques will be paired with space telescopes.

\section{CONCLUSIONS}

Astronomers for thousands of years have been trying to unravel the mysteries of the Universe, while biologists have been working on the mysteries of Life. In fact, however, Iife is an integral part and probably the finest manifestation of Nature, and therefore it is impossible to fully comprehend the Universe without understanding life's place in it.

At last we do have the science and technology to conduct serious searches for extraterrestrial life and we have already been doing so. The number and sophistication of these searches has been increasing steadily in recent years, and 1mportant technological developments anticipated for this decade promise to increase their effectiveness to much higher levels. We also have the necessary manpower, many dedicated astronomers and other scientists from around the world who are eager to undertake these tasks. The rapid swelling of the membership of our new Commission to nearly 200 attests to that. The time for this new fleld has come, and the decision of the IAU, the acknowledged spokesman for Astronomy around the world to establish this new Commission, has provided the necessary recognition and endorsement. We can expect, therefore, an exciting future for this new branch of Astronomy, the search for life (BIOS) in the Universe, which 1s rapidly becoming known as BIOASTRONOMY.

We must be aware, however, that the search for extraterrestrial 11fe is 1ikely to be an arduous task. The chances of finding extraterrestrial life, primitive or advanced, depend on how common they are and how easy it is to detect them. Primitive forms of life, judging from how fast they appeared on Earth, are probably relatively common in the Galaxy. The spectroscopic detection of their biological activities, however, in planets of other solar systems (one of the long-term objectives of our Commission) is a very difficult task that requires further technological developments. Galactic civilizations, on the other hand, are probably much easier to detect but they could be quite rare in the Galaxy, which would also make their discovery very difficult. It is even conceivable that an extensive search for extraterrestrial intelligence might ultimately lead us to the conclusion that We are the only technological civilization in the Galaxy. Even this, however, should not be taken as a failure of our efforts, because exciting as it might be to find other advanced civilizations, it is equally important to know that we are the only one in the entire Galaxy. The responsibility to preserve this unique civilization and the opportunity to infuse intelligent life into a barren Galaxy will be enormous challenges for the generations to come.

We stand at a historic cross-road. We have the technical capability, the skilled scientists, and above all the strong desire to move ahead with our search for extraterrestrial. 1ife. Let us proceed with this noble quest which will allow us to gain a far better understanding of the Universe in which we 11 ve.

ACKNOWLEDGEMENTS: On behalf of the entire membership of Commission 51, I wish to express my appreciation to Dr. Patrick Wayman, the General Secretary of the IAU during the period 1979-1982, for all his work and efforts to make this new Commission a reality. Also to all the members of the Executive Commitee for their support in this effort and for the honor of electing me the first President of Commission 51 . I am also grateful to the new Secretary General of the Union, Dr. Richard West, to our new President, Dr. R. Harbury Brown, and to the new E.C. for the support and 
interest they have already shown for our new Commission, and for appointing me the official representative of the IAU to the IAF. I also want to thank my colleagues, F. Drake, G. Gatewood, S. Gulkis, P. Horowitz, and J. Tarter for providing me with useful information which I used in the preparation of this report. In closing I want to thank the nearly 200 fellow IAU members and distinguished colleagues from related disciplines who have enthusiastically joined our new Commission. I hope our new Commission will meet the expectations of all of us.

Prof. MICHAEL D. PAPAgIANNIS *

President, IAU Commission 51

* Dept. of Astronomy, Boston University, Boston, MA 02215, USA.

Tel. (617) 353-2625. 
WORKING GROUP FOR PLANETARY SYSTEM NOMENCLATURE (NOMENCLATURE DU SYSTEME PLANETAIRE) (Committee of the Executive Committee)

Report of Meetings on 6 June 1980, 16 April 1981, 16-19 August 1982

PRESIDENT: P.M. Millman

A brief summary of the first two meetings listed above has appeared in Trans. IAU vol XVIII A, p. 669,1982 . A detailed listing of action taken at all three meetings, as approved at the General Assembly in Patras, is given herewith.

One new Latin term has been used in addition to the 32 previously listed in Trans. IAU vols. XVI B and XVII B.

COLLIS (Colles) a small h1ll or knob

The plural form is given in brackets.

The current membership in the WGPSN and in the five nomenclature task groups is listed on page 458 , this volume.

LUNAR NOMENCLATURE

Table 1. 6 names, approved as assigned to craters on the lunar surface. Positions are given in degrees of latitude and longitude.

\begin{tabular}{|c|c|c|c|}
\hline $\begin{array}{l}\text { BEALS, Carlyle S. } \\
\text { (replaces Riemann A) }\end{array}$ & $\begin{array}{l}\text { 1899-1979 } \\
\text { Canadian astronomer }\end{array}$ & $37.1 \mathrm{~N}$ & $086.0 \mathrm{E}$ \\
\hline BOS, Willem H, van den & $\begin{array}{l}1896-1974 \\
\text { Dutch astronomer }\end{array}$ & $05.3 \mathrm{~s}$ & $145.8 \mathrm{E}$ \\
\hline $\begin{array}{l}\text { JENKINS, Louise } \\
\quad(\text { replaces Schubert } \mathrm{Z})\end{array}$ & $\begin{array}{l}1888-1970 \\
\text { American astronomer }\end{array}$ & $00.7 \mathrm{~N}$ & $078.0 \mathrm{E}$ \\
\hline KAO, Ping-Tse & $\begin{array}{l}1888-1970 \\
\text { Taiwanese astronomer }\end{array}$ & $06.7 \mathrm{~s}$ & $087.8 \mathrm{E}$ \\
\hline $\begin{array}{l}\text { KELDYSH, Mstislav V. } \\
\text { (replaces Hercules A) }\end{array}$ & $\begin{array}{l}\text { 1911-1978 } \\
\text { Soviet mathematician }\end{array}$ & $51.5 \mathrm{~N}$ & $044.0 \mathrm{E}$ \\
\hline RECHT, Albert & $\begin{array}{l}\text { 1899-1962 } \\
\text { American astronomer }\end{array}$ & $09.8 \mathrm{~N}$ & $124.0 \mathrm{E}$ \\
\hline
\end{tabular}

MERCURY NOMENCLATURE

Errata: YAKOVLEV, a crater on Mercury, 1isted at bottom of p. 292, Trans. IAU vol. XVII B, 1980 , should be corrected to -

BARMA, Ivan Postnik Yakovlev ( 16 th cen) Russian architect 
VENUS NOMENCLATURE

Table 2. 66 names, approved as assigned to features on the surface of Venus. Approximate locations are given in degrees of latitude and longitude.

\section{CRATERS}

COLETTE

EARHART

NIGHTINGALE

PAVLOVA

SACAJAWEA

\section{CHASMATA}

ARTEMIS

DALI

DEVANA

DIANA

GANIS

HECATE

HENG-O

IX CHEL

JUNO

KUANJA

PARGA

QUILLA

VIRAVA

DORSUM

JUNO

LINEAE

ANTIOPE

GUOR

HARIASA

HIPPOLYTA

KARA

LAMPEDO

MOLPADIA

VIHANSA
(1873-1954) French writer

(1897-1937) American flyer

(1820-1910) English nurse

(1881-1931) Russian classical dancer

(1786-1812) Shoshone Indian guide

Greek goddess of hunt/moon

Georgian goddess of hunt

Czechoslovakian goddess of hunt

Roman goddess of hunt/moon

Western Lapp forest maiden

Greek moon goddess

Chinese moon goddess

Aztec wife of the Sun god

Roman sky goddess, consort of Jupiter

Mbundu goddess of hunt

Samoyed forest spirit

Inca moon goddess

Mordvin forest mother

Roman sky goddess, consort of Jupiter

Greek amazon

Northern European valkyrie

German war goddess

Greek amazon

Icelandic valkryie, a sweet singer

Scythian amazon queen

Greek amazon

German war goddess

$\begin{array}{ll}65 \mathrm{~N} & 322 \mathrm{E} \\ 72 \mathrm{~N} & 136 \mathrm{E} \\ 62 \mathrm{~N} & 132 \mathrm{E} \\ 14 \mathrm{~N} & 040 \mathrm{E} \\ 63 \mathrm{~N} & 335 \mathrm{E}\end{array}$

$30 \mathrm{~S}-42 \mathrm{~S}$

121E-145E

$17 \mathrm{~S}-21 \mathrm{~S}$

155E-180E

00

$289 \mathrm{E}$

$15 \mathrm{~s}$

$150 \mathrm{E}$

$19 \mathrm{~N}-10 \mathrm{~N} \quad 188 \mathrm{E}-200 \mathrm{E}$

$15 \mathrm{~N}-18 \mathrm{~N} \quad 230 \mathrm{E}-245 \mathrm{E}$

$00-10 \mathrm{~N} \quad 350 \mathrm{E}-000$

09S-11S 071E-078E

32S 102E-120E

10S-16S 097E-112E

08S-4IS 222E-321E

32S-38S $\quad 118 \mathrm{E}-135 \mathrm{E}$

13S-17S 117E-132E

$32 \mathrm{~S}-35 \mathrm{~S} \quad 085 \mathrm{E}-095 \mathrm{E}$

$\begin{array}{ll}40 \mathrm{~S} & 350 \mathrm{E} \\ 20 \mathrm{~N} & 000 \\ 19 \mathrm{~N} & 015 \mathrm{E} \\ 42 \mathrm{~S} & 345 \mathrm{E} \\ 44 \mathrm{~S} & 306 \mathrm{E} \\ 57 \mathrm{~N} & 295 \mathrm{E} \\ 48 \mathrm{~S} & 359 \mathrm{E} \\ 54 \mathrm{~N} & 020 \mathrm{E}\end{array}$


Table 2. (Cont'd)

MONTES

\begin{tabular}{|c|c|c|c|}
\hline GULA & Babylonian earth mother & $22 \mathrm{~N}$ & 000 \\
\hline INNINI & $\begin{array}{l}\text { Babylonian earth mother, worshipped at } \\
\text { Kish }\end{array}$ & $35 \mathrm{~S}$ & $329 \mathrm{E}$ \\
\hline MAAT & Egyptian goddess of truth and justice & $02 \mathrm{~S}$ & $194 \mathrm{E}$ \\
\hline NOKOMIS & Algonquin earth mother & $20 \mathrm{~N}-18 \mathrm{~N}$ & $189 \mathrm{E}-191 \mathrm{E}$ \\
\hline $\begin{array}{l}\text { OZZA } \\
\text { (former 1y Gaea) }\end{array}$ & Persian goddess honored by the Koreishies & $05 \mathrm{~N}$ & $200 \mathrm{E}$ \\
\hline $\begin{array}{l}\text { SAPAS } \\
\text { (formerly Ceres) }\end{array}$ & Phoenician goddess & $08 \mathrm{~N}$ & $188 \mathrm{E}$ \\
\hline SIF & Teutonic goddess, Thor's wife & $21 N$ & $352 \mathrm{E}$ \\
\hline TEFNUT & Egyptian goddess of dew or rain & $39 \mathrm{~s}$ & $304 \mathrm{E}$ \\
\hline USHAS & Indian goddess of dawn & $25 S$ & $323 E$ \\
\hline \multicolumn{4}{|l|}{ PATERAE } \\
\hline CLEOPATRA & (69 BC-30 BC) Egyptian queen & $67 \mathrm{~N}$ & $009 \mathrm{E}$ \\
\hline THEODORA & $\begin{array}{l}\text { (c.500-548) Byzantine empress, wife of } \\
\text { Justinian I }\end{array}$ & $23 \mathrm{~N}$ & $280 \mathrm{E}$ \\
\hline \multicolumn{4}{|l|}{ PLANITIAE } \\
\hline AINO & Finnish heroine and water sprite & $45 S$ & $090 \mathrm{E}$ \\
\hline ATALANTA & Greek mythology, a swift-footed huntress & $54 \mathrm{~N}$ & $162 \mathrm{E}$ \\
\hline GUINEVERE & British queen, wife of Arthur & $40 \mathrm{~N}$ & $310 \mathrm{E}$ \\
\hline HELEN & $\begin{array}{l}\text { Wife of Menalaus in ancient Greece } \\
(\mathrm{c} .1100 \mathrm{BC})\end{array}$ & $55 \mathrm{~S}$ & $255 \mathrm{E}$ \\
\hline LAVINIA & Roman wife of Aeneas & $45 S$ & $350 \mathrm{E}$ \\
\hline LEDA & $\begin{array}{l}\text { Greek mythology, mother of Helen } \\
\text { and Castor }\end{array}$ & $45 \mathrm{~N}$ & $065 \mathrm{E}$ \\
\hline NAVKA & Sma11 water nymph & $08 \mathrm{~S}$ & $315 \mathrm{E}$ \\
\hline NIOBE & $\begin{array}{l}\text { Greek, her } 12 \text { children killed by Artemis } \\
\text { and Apollo }\end{array}$ & $38 \mathrm{~N}$ & $132 \mathrm{E}$ \\
\hline RUSALKA & Russian mermaid & $10 \mathrm{~N}-10 \mathrm{~S}$ & $160 E-185 E$ \\
\hline SEDNA & Inuit whose fingers became seals and & $40 \mathrm{~N}$ & $335 \mathrm{E}$ \\
\hline
\end{tabular}

REGIONES

$\begin{array}{llcc}\text { ASTERIA } & \text { Greek titaness } & 18 \mathrm{~N}-30 \mathrm{~N} & 228 \mathrm{E}-270 \mathrm{E} \\ \text { ATLA } & \text { Norse giantess, mother of Heinda11 } & 20 \mathrm{~N}-05 \mathrm{~S} & 185 \mathrm{E}-210 \mathrm{E} \\ \text { BELL } & \text { English giantess } & 25 \mathrm{~N}-35 \mathrm{~N} & 045 \mathrm{E}-055 \mathrm{E} \\ \text { EISILA } & \text { Greek giantess } & 10 \mathrm{~N}-25 \mathrm{~N} & 350 \mathrm{E}-050 \mathrm{E} \\ \text { IMDR } & \text { Norse giantess } & 42 \mathrm{~S} & 211 \mathrm{E} \\ \text { METIS } & \text { Greek titaness } & 72 \mathrm{~N} & 245 \mathrm{E}-255 \mathrm{E}\end{array}$


Table 2. (Cont'd)

\begin{tabular}{llcc}
\hline MNEMOSYNE & Greek titaness & $68 \mathrm{~N}$ & $275 \mathrm{E}-285 \mathrm{E}$ \\
OVDA & Marijian titaness & $05 \mathrm{~N}-10 \mathrm{~S}$ & $080 \mathrm{E}-108 \mathrm{E}$ \\
PHOEBE & Greek titaness & $10 \mathrm{~N}-20 \mathrm{~S}$ & $275 \mathrm{E}-300 \mathrm{E}$ \\
TELLUS & Greek titaness & $35 \mathrm{~N}$ & $080 \mathrm{E}$ \\
TETHUS & Greek titaness & $55 \mathrm{~N}$ & $100 \mathrm{E}$ \\
THEMIS & Greek titaness & $37 \mathrm{~S}-40 \mathrm{~S}$ & $275 \mathrm{E}-310 \mathrm{E}$ \\
THETIS & Greek titaness & $02 \mathrm{~S}-15 \mathrm{~S}$ & $118 \mathrm{E}-140 \mathrm{E}$ \\
ULFRUN & Norse giantess & $18 \mathrm{~N}-03 \mathrm{~S}$ & $220 \mathrm{E}-230 \mathrm{E}$ \\
RUPES & & $03 \mathrm{~N}-08 \mathrm{~N}$ & $059 \mathrm{E}-075 \mathrm{E}$ \\
HESTIA & Greek hearth goddess & $48 \mathrm{~N}-53 \mathrm{~N}$ & $305 \mathrm{E}-325 \mathrm{E}$ \\
UT & Siberian, Turco-Tatar goddess of hearth & $55 \mathrm{~N}-65 \mathrm{~N}$ & $295 \mathrm{E}-355 \mathrm{E}$ \\
VESTA & Roman hearth goddess & & \\
TERRA & & $50 \mathrm{~S}-65 \mathrm{~S}$ & $285 \mathrm{E}-110 \mathrm{E}$ \\
\hline LADA & Serbian goddess of love & &
\end{tabular}

MARS NOMENCLATURE

Table 3. 44 names, approved as assigned to features on the surface of Mars . Positions have been given in degrees of latitude and longitude.

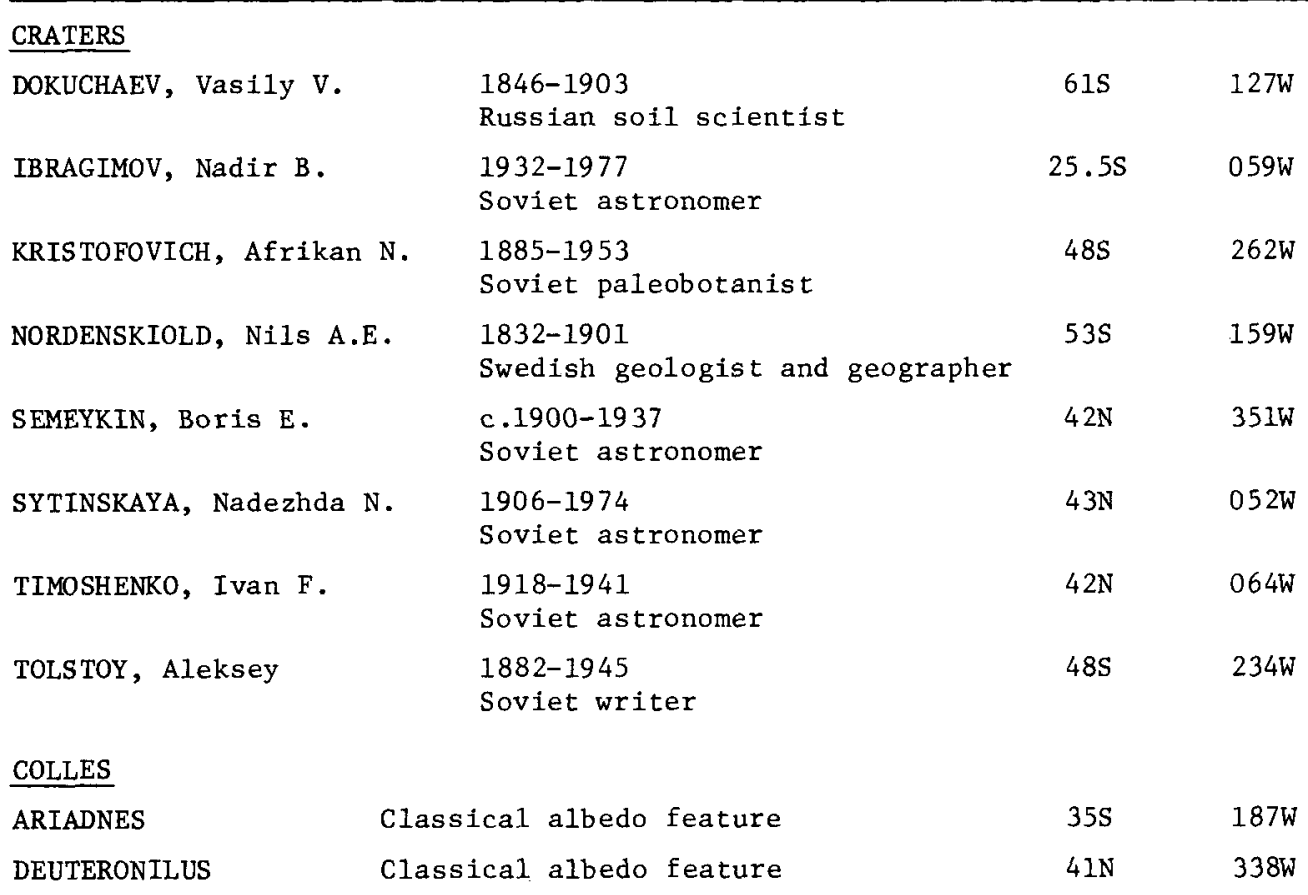


Table 3. (Cont'd)

\section{CHAOS}

ARS INOES

Daughter of Ptolomy Lagus and Bernice, $08 \mathrm{~S}$

$028 \mathrm{~W}$

EOS albedo feature

PYRRHAE

Greek name of Aurora, albedo feature

16S-19S 043W-050W

Daughter of Pandora, albedo feature

$11 \mathrm{~S}$

$029 \mathrm{~W}$

DORS UM

CERBERUS

Classical albedo feature

09S-18S 252W-258W

FOS SAE

CALYDON

Son of Ares and Astynome

$07.5 \mathrm{~S} \quad 087 \mathrm{~W}-093 \mathrm{~W}$

COLOE

Classical albedo feature

$32 \mathrm{~N}-41.5 \mathrm{~N} 294 \mathrm{~W}-307 \mathrm{~W}$

ISMENIAE

Classical albedo feature

$37 \mathrm{~N}-42 \mathrm{~N} \quad 315 \mathrm{~W}-337 \mathrm{~W}$

NIA

River Gambia, West Africa

13S-15S 070W-074W

OTI

Classical albedo feature

07S-I2S 115W-118W

TITHONIAE

Classical albedo feature

01S-03S $034 \mathrm{~W}-047 \mathrm{~W}$

LABYRINTHUS

ADAMAS

Classical albedo feature

$30 \mathrm{~N}-42 \mathrm{~N} \quad 247 \mathrm{~W}-257 \mathrm{~W}$

MENSAE

CANDOR

Classical albedo feature

$06 \mathrm{~S}$

$028 \mathrm{~W}$

HEBES

Classical albedo feature

$01 \mathrm{~S}$

$032 \mathrm{~W}$

MONTES

EREBUS

Classical albedo feature

$30 \mathrm{~N}-42 \mathrm{~N}$

$170 \mathrm{~W}-180 \mathrm{~W}$

GERYON

Classical albedo feature

$07 \mathrm{~S}-08 \mathrm{~S} \quad 078 \mathrm{~W}-084 \mathrm{~W}$

PATERAE

MEROE

NILI

Classical albedo feature

$07 \mathrm{~N}$

$291 W$

Classical albedo feature

$09 \mathrm{~N}$

$293 \mathrm{~W}$

PLANUM

DAEDALIA

Classical albedo feature

$17 \mathrm{~S}-23 \mathrm{~S} \quad 115 \mathrm{~W}-125 \mathrm{~W}$

RUPES

AMENTHES

Classical albedo feature

$00-04 \mathrm{~N} 248 \mathrm{~W}-252 \mathrm{~W}$

MORPHEOS

Classical albedo feature

$36 \mathrm{~S}-39 \mathrm{~S} \quad 231 \mathrm{~W}-240 \mathrm{~W}$

SCOPULUS

OENOTRIA

Classical albedo feature

$15 S-10 S \quad 270 W-292 W$

VALLES

ARNUS

(replaces Arena Rupes)

River in Tuscany, Italy

$18.5 \mathrm{~N}$

$290 \mathrm{~W}$

BRAZOS

River in Texas, USA

$02.5 S-06 S 339 W-344 W$ 
Table 3. (Cont'd)

\begin{tabular}{lllc} 
DRAVA & River in Yugoslavia & $47 \mathrm{~S}-56 \mathrm{~S}$ & $192 \mathrm{~W}-197 \mathrm{~W}$ \\
EVROS & River in Greece & $12 \mathrm{~S}-13 \mathrm{~S}$ & $343 \mathrm{~W}-349 \mathrm{~W}$ \\
GRANICUS & Ancient river in Turkey & $27 \mathrm{~N}-30 \mathrm{~N}$ & $225 \mathrm{~W}-231 \mathrm{~W}$ \\
HEBRUS & Ancient river in Greece & $17 \mathrm{~N}-22 \mathrm{~N}$ & $232 \mathrm{~W}-234 \mathrm{~W}$ \\
INDUS & River in Pakistan & $17 \mathrm{~N}-21 \mathrm{~N}$ & $317 \mathrm{~W}-322 \mathrm{~W}$ \\
LOIRE & River in France & $17.5 \mathrm{~S}-22 \mathrm{~S}$ & $014 \mathrm{~W}-017.5 \mathrm{~W}$ \\
LICUS & Ancient river in France & $01 \mathrm{~S}-05 \mathrm{~S}$ & $233 \mathrm{~W}-234 \mathrm{~W}$ \\
LOUROS & River in Greece & $08 \mathrm{~S}-09.5 \mathrm{~S} 079 \mathrm{~W}-086 \mathrm{~W}$ \\
NAKTONG & River in Korea & $00-09 \mathrm{~N}$ & $330 \mathrm{~W}-321 \mathrm{~W}$ \\
REULL & Word for planet in Gaelic & $37 \mathrm{~S}-43 \mathrm{~S}$ & $248 \mathrm{~W}-265 \mathrm{~W}$ \\
SCAMANDER & Ancient river near Troy, Turkey & $14 \mathrm{~N}-18 \mathrm{~N}$ & $330 \mathrm{~W}-331 \mathrm{~W}$ \\
\hline
\end{tabular}

OUTER SOLAR SYSTEM NOMENCLATURE

The following names* were approved for recently discovered (or more completely observed) satelitites of Jupiter and Saturn:-

\begin{tabular}{cc} 
Permanent Designation & Temporary Designation \\
\hline Jupiter XIV & $1979 \mathrm{~J} 2$ \\
Saturn X & $1980 \mathrm{~S} 1$ \\
Saturn XI & $1980 \mathrm{~S} 3$ \\
Saturn XIII & $1980 \mathrm{~S} 13$ \\
Saturn XIV & $1980 \mathrm{~S} 25$
\end{tabular}

The following nomenclature was approved for divisions or gaps in the rings of Saturn. The first two names are traditional, as used for divisions seen by earth-based visual observers. The last three are applied to prominent narrow gaps, as revealed by the Voyager 2 photography. (See figure on page 345)

\footnotetext{
* Following detailed discussions in Patras among members of Commissions 6 and 20 and the WGPSN it was suggested that three names, orginally recommended by the WGPSN (i.e. ADRASTEA for $1979 \mathrm{J1}$, METIS for $1979 \mathrm{~J} 3$, ATLAS for $1980 \mathrm{~S} 28$ ), be considered provisional until further information re the orbital elements of these three objects had been made generally available. These discussions also called attention to the fact that the IAU had not formally assigned the responsibility for the various steps that must be taken following the discovery of new bodies in the solar system. A joint meeting of representatives from the three IAU units noted above was held and a plan for future action was drawn up. This plan was later endorsed by the Executive Committee of the IAU and the General Assembly. It is reproduced in the Astronomer's Handbook, Section 4, p. 383, this volume, and in the IAU Information Bulletin No. 49, January 1983.
} 
CASSINI, Giovanni D. (1625-1712) - the prominent division between the A ring and the B ring, approx. radius $119,000 \mathrm{~km}$.

ENCKE, Johann F. (1791-1865)

- the widest division in the A ring, approx. radius $133,500 \mathrm{~km}$.

MAXWELL, James C. (1831-1879) - the widest gap in the C ring, approx. radius $87,500 \mathrm{~km}$.

HUYGENS, Christiaan (1629-1695) - the gap at the inner edge of the Cassini division, approx. radius $117,500 \mathrm{~km}$.

KEELER, James E. (1857-1900)

- the gap near the outer edge of the A ring, approx. radius $136,500 \mathrm{~km}$.

Table 4. 7 names, approved as assigned to features on the surfaces of 2 satellites of Jupiter. Approximate positions are given in degrees of latitude and longitude.

IO NOMENCLATURE

PATERAE

$\begin{array}{llll}\text { HUO SHEN } & \text { Chinese god of fire } & 15 \mathrm{~S} & 329 \mathrm{~W} \\ \text { KIBERO } & \text { Underworld toad who gives mankind fire } & 12 \mathrm{~S} & 306 \mathrm{~W} \\ \text { MBALI } & \text { Pygmy word for fire } & 32 \mathrm{~S} & 007 \mathrm{~W} \\ \text { SHOSHU } & \text { Caucasian patron of fire } & 19.5 \mathrm{~S} & 324.5 \mathrm{~W} \\ \text { TALOS } & \text { A smith, nephew of Daedalus } & 26 \mathrm{~S} & 356 \mathrm{~W} \\ \text { TAW } & \text { Monguor word for fire or hearth } & 33.5 \mathrm{~S} & 000.5 \mathrm{~W}\end{array}$

GANYMEDE NOMENCLATURE

CRATER

ENKIDU

Friend of Gilgamesh

$18 \mathrm{~S}$

$322 \mathrm{~W}$

Table 5. 190 names, approved as assigned to features on the surfaces of 9 satellites of Saturn. Approximate positions are given in degrees of latitude and longitude.

JANUS NOMENCLATURE

\section{CRATERS}

CASTOR One of the Dioskuroi, a tamer of horses

IDAS

Cousin of Gemini

LYNCEUS

Cousin of Gemini

PHOIBE

Daughter of Leukippos

EPIMETHEUS NOIENCLATURE

CRATERS

HILAIREA

Wife of Pollux

POLLUX

Latin name for Polydeukes, Castor's twin 


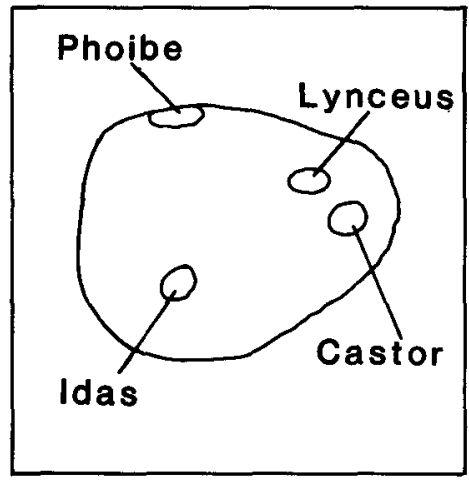

JANUS

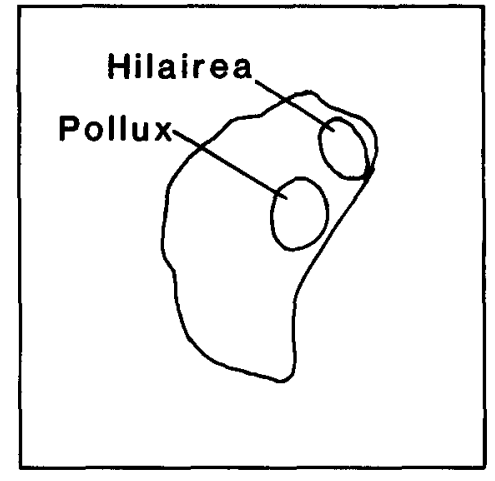

EPIMETHEUS

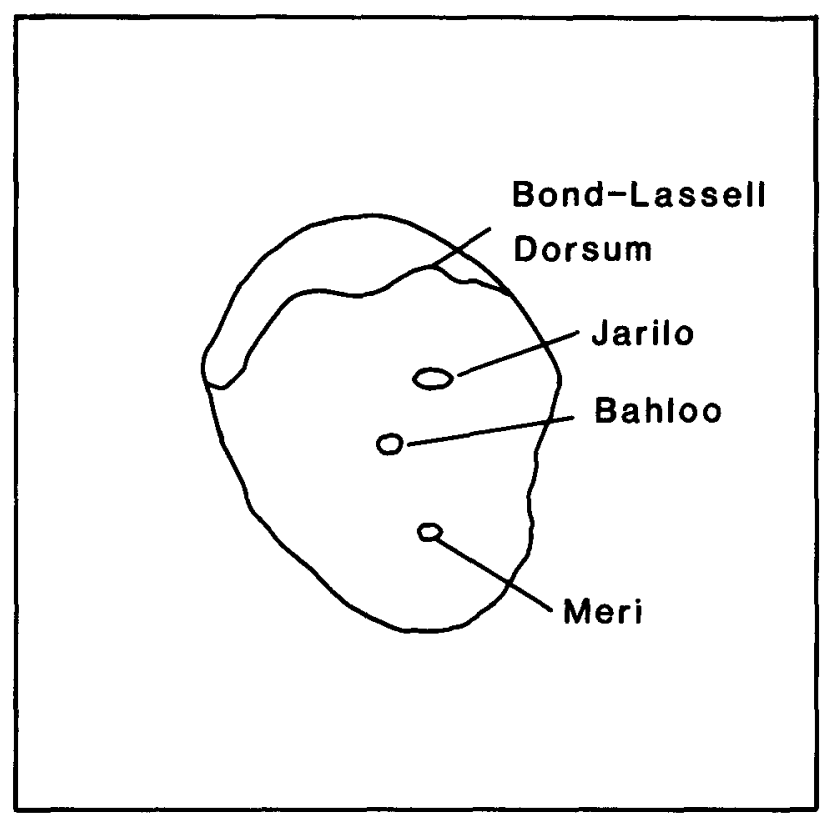

HYPERION 
Table 5. (Cont'd)

MIMAS NOMENCLATURE

CRATERS

ACCOLON

Arthur's companion, jousted with Arthur

$68 \mathrm{~S}$

$166 \mathrm{~W}$

ARTHUR

King of the Round Table assemblage

$35 \mathrm{~S}$

$190 \mathrm{~W}$

BALIN

Knight of "matchless courage and virtue"

$22 \mathrm{~N} \quad 082 \mathrm{~W}$

BAN

King of Benwick, father of Sir Launcelot

$47 \mathrm{~N}$

149W

BEDIVERE

Knight of the Round Table

$10 \mathrm{~N}$

$145 \mathrm{~W}$

BORS

King of Gaul

$45 \mathrm{~N}$

$165 \mathrm{~W}$

DYNAS

Knight of the Round Table

$08 \mathrm{~N}$

$075 \mathrm{~W}$

ELAINE

Daughter of King Pelles

$102 \mathrm{~W}$

GAHERIS

01der son of King Lot

$44 \mathrm{~N}$

$287 \mathrm{~W}$

GAIAHAD

Bastard son of Launcelot and Elaine

$46 \mathrm{~S}$

$135 \mathrm{~W}$

GARETH

Youngest son of King Lot

280W

GAWAIN

Eldest son of King Lot

$254 \mathrm{~W}$

GWYNEVERE

Queen, wife of Arthur and lover of Launcelot

475

$44 S$

$312 W$

HERSCHEL, William

(1738-1822) Astronomer who discovered Mimas

$12 \mathrm{~S}$

$104 \mathrm{~W}$

IGRAINE

Wife of Uther, mother of Arthur

$225 \mathrm{~W}$

ISEURT

Loved by Tristram

$035 \mathrm{~W}$

KAY

Royal senesche1 at Arthur's court

$116 \mathrm{~W}$

LAMEROK

Pellinore's son

$283 \mathrm{~W}$

LAUNCELOT

King Arthur's favorite knight

$317 \mathrm{~W}$

LOT

Leader of the rebel kings of North and West

$227 \mathrm{~W}$

MARK

King of Cornwall

$297 \mathrm{~W}$

Magician and prophet, Arthur's mentor

$215 \mathrm{~W}$

Arthur's bastard son and mortal enemy

$213 \mathrm{~W}$

Arthur's half sister, an enchantress

$240 \mathrm{~W}$

Saracen enemy of Tristram

$157 \mathrm{~W}$

King who pursued the questing beast

$128 \mathrm{~W}$

Pure Knight, finds the Holy Grail

$171 \mathrm{~W}$

Saves Iseult, falls in love with her

$026 \mathrm{~W}$

Ruler of all Britain, Arthur's father

$244 \mathrm{~W}$

CHASMATA

AVALON
CAMELOT
OETA
OSSA
PANGEA
PELION
TINTAGEL

Arthurian paradise

$20 \mathrm{~N}-57 \mathrm{~N} \quad 160 \mathrm{~W}-120 \mathrm{~W}$

Home of the Round Table assemblage

25S-60S $000-045 \mathrm{~W}$

Shook by Titan in war with olympians

$10 \mathrm{~N}-35 \mathrm{~N}$

$130 \mathrm{~W}-105 \mathrm{~W}$

Mt. Pelion piled on top of it

$10 \mathrm{~S}-30 \mathrm{~S}$

$305 \mathrm{~W}-280 \mathrm{~W}$

Picked up by Titan in war with gods

$25 S-55 S$

$340 \mathrm{~W}-290 \mathrm{~W}$

Piled up with Mt. Ossa in war with gods

$20 \mathrm{~S}-25 \mathrm{~S}$

$235 \mathrm{~W}-200 \mathrm{~W}$

Home of Igraine, Arthur's mother

43S-60s

$235 \mathrm{~W}-190 \mathrm{~W}$ 
Table 5. (Cont'd)

ENCELADUS NOMENCLATURE

CRATERS

AHMAD

ALADDIN

ALI BABA

DALILAH

DUBAN

DUNYAZAD

GHARIB

JULNAR

MUSA

PERI-BANU

SALIH

SAMAD

SHAHRAZAD

SHAHRYAR

SINDBAD

FOSSAE

BASSORAH

DARYABAR

ISBANIR

PLANITIAE

DIYAR

SARANDIB

SULCI

HARRAN

SAMARKAND

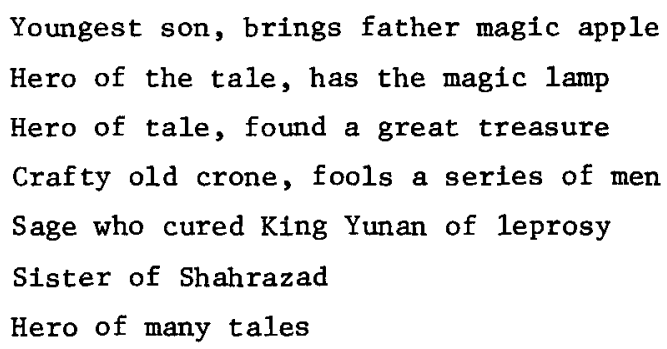

TETHYS NOMENCLATURE

\section{CRATERS}

$\begin{array}{lllr}\text { AJAX } & \text { Greek hero, second only to Achilles } & 30 \mathrm{~S} & 285 \mathrm{~W} \\ \text { ANTICLEIA } & \text { Mother of Odysseus } & 55 \mathrm{~N} & 038 \mathrm{~W} \\ \text { ANTINOUS } & \text { Chief of Penelope's wooers } & 62 \mathrm{~S} & 275 \mathrm{~W} \\ \text { ARETE } & \text { Wife of Alcinous, mother of Nausicaa } & 04 \mathrm{~S} & 300 \mathrm{~W} \\ \text { CIRCE } & \text { Changed Odysseus' companions into swine } & 08 \mathrm{~S} & 049 \mathrm{~W} \\ \text { ELPENOR } & \text { Follower of Odysseus } & 54 \mathrm{~N} & 268 \mathrm{~W} \\ \text { EUMAEUS } & \text { Faithful swineherd who greets Odysseus } & 27 \mathrm{~N} & 047 \mathrm{~W}\end{array}$


Table 5. (Cont'd)

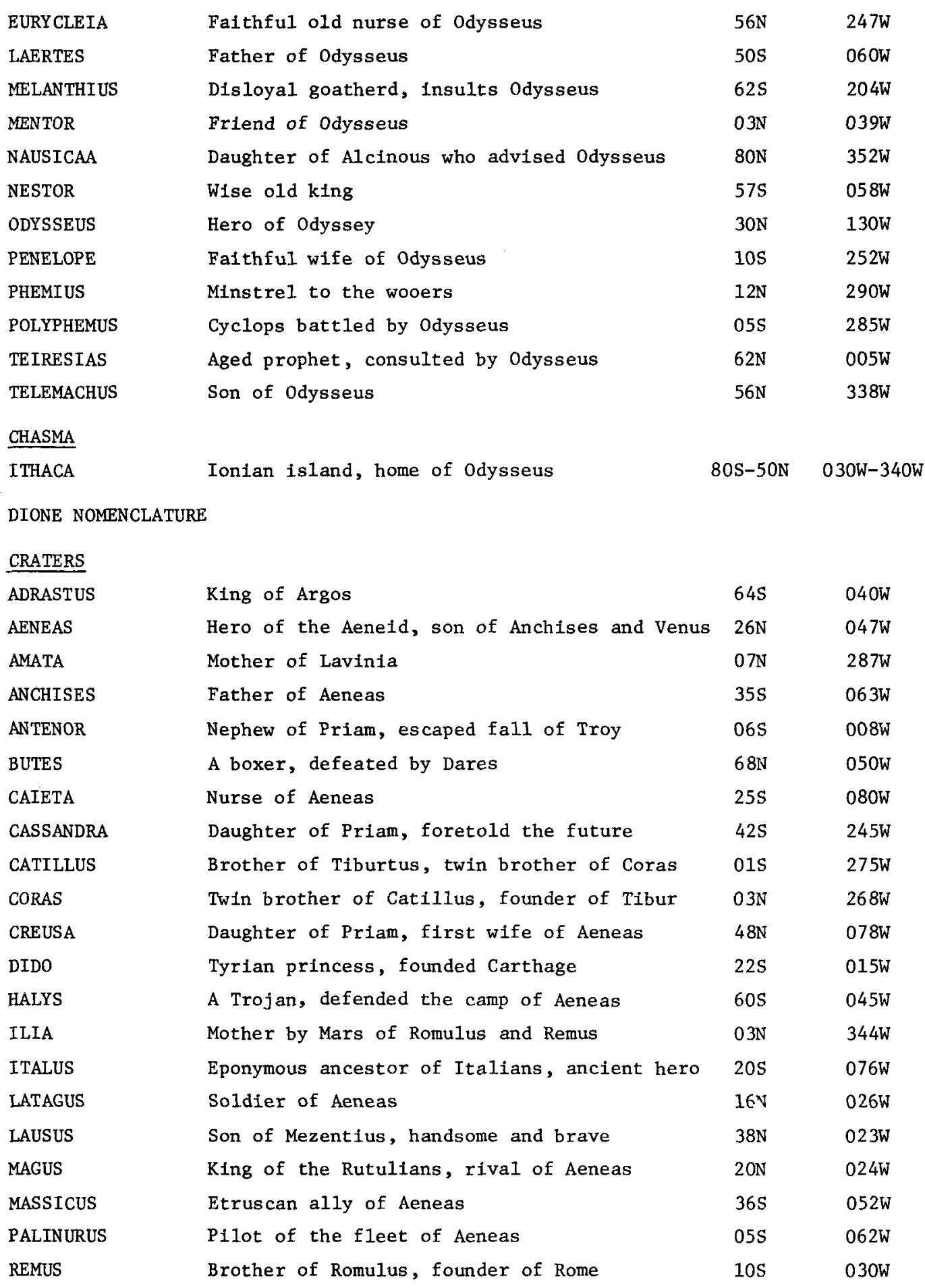


Table 5. (Cont'd)

\begin{tabular}{|c|c|c|c|}
\hline RIPHEUS & A Trojan who fought beside Aeneas at Troy & $56 \mathrm{~S}$ & 029W \\
\hline ROMULUS & Mythical founder of Rome circa $754 \mathrm{BC}$ & $08 \mathrm{~S}$ & $024 \mathrm{~W}$ \\
\hline SABINUS & Fabled ancestor of the Sabines & $44 S$ & $190 \mathrm{~W}$ \\
\hline TURNUS & Rutulian king, rival of Aeneas for Lavinia & $21 \mathrm{~N}$ & $342 W$ \\
\hline \multicolumn{4}{|l|}{ CHASMATA } \\
\hline LARISSA & Town in Thessaly, Achilles' native region & $20 N-48 N$ & $015 \mathrm{~W}-065 \mathrm{~W}$ \\
\hline LATIUM & The Trojans' promised land in Italy & $03 N-45 N$ & $064 W-075 W$ \\
\hline PALATINE & One of the seven hills of Rome & $55 S-73 S$ & $075 \mathrm{~W}-320 \mathrm{~W}$ \\
\hline TIBUR & Ancient town of Italy near Rome & $48 \mathrm{~N}-80 \mathrm{~N}$ & $060 \mathrm{~W}-080 \mathrm{~W}$ \\
\hline \multicolumn{4}{|l|}{ LINEAE } \\
\hline CARTHAGE & An ancient Etruscan city & $20 N-10 N$ & $337 \mathrm{~W}-310 \mathrm{~W}$ \\
\hline PADUA & City in Italy, founded by Antenor & $05 N-40 S$ & $245 W-190 W$ \\
\hline PALATINE & One of the seven hills of Rome & $10 S-55 s$ & $285 \mathrm{~W}-320 \mathrm{~W}$ \\
\hline
\end{tabular}

RHEA NOMENCLATURE

\section{CRATERS}

AANANIN

Korean god of the heavens

ADJUA

Mythical heroine, ancestor of Ulci tribe

AGUNUA

Melanesian god who made sea, land and people $70 \mathrm{~N}$

$065 \mathrm{~W}$

AMETA

Indonesian ancestor whose blood made Hainuwele 59N

$014 \mathrm{~W}$

ARUNAKA

Inca god, creator of a11 things

$14 \mathrm{~S}$

$021 \mathrm{~W}$

ATUM

Creator god of Heliopolos, son of Ptah

$45 \mathrm{~S}$

000

BULAGAT

Mythological ancestor of Buriat tribe

$35 \mathrm{~S}$

$014 \mathrm{~W}$

BUMBA

Bushongo god, vomited up sun, moon, men, etc. $70 \mathrm{~N}$

$040 \mathrm{~W}$

BURKHAN

Siberian god who created world

$69 \mathrm{~N}$

$288 \mathrm{~W}$

CON

DJULI

Coastal creator god

ELLYAY

Ukrainian first man, ancestor of the people

$26 \mathrm{~S}$

046W

FARO

Yakutian ancestor of the people

HAIK

Mande, sacrificial killing purified Earth

$52 \mathrm{~N}$

$121 \mathrm{~W}$

Mythological ancestor of Armenian people

HAOSO

Manchurian creator of all things

$09 \mathrm{~N}$

$008 \mathrm{~W}$

HELLER

Auracanin creator of men and civilization

$09 \mathrm{~N}$

$310 \mathrm{~W}$

IRACA

IZANAGI

IZANAMI

Incan creator god who became the moon

$45 \mathrm{~N}$

$120 \mathrm{~W}$

Japanese mythology, creator god

Japanese creator goddess, sister of Izanagi $46 \mathrm{~S}$

JUMO

Marijtan sky god

$56 \mathrm{~N}$

$065 \mathrm{~W}$

KARORA

Aranda, Australian ancestor

$07 \mathrm{~N}$

KHADO 
Table 5. (Cont'd)

\begin{tabular}{|c|c|c|c|}
\hline KIHO & Tuamotu, Society Islands, made land and sea & $10 \mathrm{~s}$ & $354 \mathrm{~W}$ \\
\hline KUMPARA & Jivaro, Ecuador, creator god & $11 \mathrm{~N}$ & $321 W$ \\
\hline LEZA & Tonga, originator of conditions of life & 19S & $304 \mathrm{~W}$ \\
\hline LOWA & Marsha11 Islands, great creator god & $45 \mathrm{~N}$ & $009 \mathrm{~W}$ \\
\hline MALUNGA & Yao, Bantu, creator god and progenitor god & $74 \mathrm{~N}$ & $049 \mathrm{~W}$ \\
\hline MANOID & Negrito, Malay Peninsula, progenitor goddess & $33 \mathrm{~N}$ & $002 \mathrm{~W}$ \\
\hline MELO & Minyong, India, the original man & $51 \mathrm{~s}$ & $006 \mathrm{~W}$ \\
\hline MUBAT & Tibetan heavenly god & $61 \mathrm{~N}$ & $011 W$ \\
\hline NUM & Samoyed god of heaven & $23 \mathrm{~N}$ & 093W \\
\hline ORMAZD & Persian progenitor god of light & $62 \mathrm{~N}$ & $052 \mathrm{~W}$ \\
\hline $\mathrm{PAN} \mathrm{KU}$ & Miao creator of all things & $72 \mathrm{~N}$ & $115 \mathrm{~W}$ \\
\hline PEDN & Negrito, Malay Peninsula, god, created men & $48 \mathrm{~N}$ & $340 \mathrm{~W}$ \\
\hline QAT & New Hebrides, Melanesia, formed men from trees & .235 & $347 W$ \\
\hline SHOLMO & Buriat, Siberia, devil, created evil creatures & $13 \mathrm{~N}$ & $340 \mathrm{~W}$ \\
\hline TAAROA & Tahitian creator god & $14 \mathrm{~N}$ & 099w \\
\hline THUNUPA & Incan god, creator of all things & $51 \mathrm{~N}$ & $015 W$ \\
\hline TIKA & Supreme Abkhaz being & $25 \mathrm{~N}$ & $087 W$ \\
\hline TORE & Pygmie lord of world, creator of all things & $01 \mathrm{~N}$ & $335 W$ \\
\hline TOROM & Ostyah sky god & $68 \mathrm{~S}$ & $345 W$ \\
\hline UKU & Estonian super-god & $85 \mathrm{~N}$ & $115 \mathrm{~W}$ \\
\hline WHAN IN & Korean god, creator of all things & $74 \mathrm{~N}$ & $121 W$ \\
\hline WURAKA & Kakadu, Australla, ancestor of all people & $28 \mathrm{~N}$ & $357 \mathrm{~W}$ \\
\hline XAMBA & Supreme Bushman, creator of all things & $04 \mathrm{~N}$ & $347 \mathrm{~W}$ \\
\hline $\mathrm{XU}$ & Bushman, creator & $61 \mathrm{~N}$ & 070W \\
\hline YU-TI & Supreme primal Chinese god & $55 \mathrm{~N}$ & $085 \mathrm{~W}$ \\
\hline \multicolumn{4}{|l|}{ CHASMATA } \\
\hline KUN LUN & Chinese mythology, home of the immortals & $37 \mathrm{~N}-50 \mathrm{~N}$ & $275 W-300 W$ \\
\hline PU CHOU & Mountain attacked by Kung Chung, sky fell & $10 \mathrm{~N}-35 \mathrm{~N}$ & $085 \mathrm{~W}-115 \mathrm{~W}$ \\
\hline
\end{tabular}

HYPERION NOMENCLATURE

\section{CRATERS}

BAHLOO The moon, maker of girl babies

HELIOS Son of Hyperion, the Greek sun god

JARILO East-Slavic god of sun, fertility and love

MERI Bororo folk hero, the sun

DORSUM

BOND-LASSELI G.W. Bond and Wm. Lassell, discoverers of Hyperion in 1848 
Table 5. (Cont'd)

IAPETUS NOMENCLATURE

CRATERS

ALMERIC

BALIGANT

One of 12 peers, killed by Marsilion

$53 \mathrm{~N}$

$274 \mathrm{~W}$

BASAN

BERENGER

BESGUN

CHARLEMAGNE

GEBOIN

Emir of Babylon, assisted Marsilion in war

$15 \mathrm{~N}$

$225 \mathrm{~W}$

French baron, brother of Basilie

$30 \mathrm{~N} \quad 197 \mathrm{~W}$

One of 12 peers, killed by Grandoyne

$59 \mathrm{~N} \quad 220 \mathrm{~W}$

Chief cook for Charlemagne's army

$72 \mathrm{~N}$

296W

King of France who conquered Spanish towns

$54 \mathrm{~N}$

266W

GODEFROY

Guarded French dead in Charlemagne's army

$56 \mathrm{~N}$

$175 \mathrm{~W}$

GRANDOYNE

Charlemagne's defender, bearer of gonfalcon

$253 \mathrm{~W}$

Son of Cappadocian king Capuel

$215 \mathrm{~W}$

HAMON

$18 \mathrm{~N}$

$271 W$

LORANT

MARSILION

French commander against Baligant

$165 \mathrm{~W}$

Saracen king of Spain, vassal of Baligant

$177 \mathrm{~W}$

MILON

Guarded French dead in Charlemagne's army

$270 \mathrm{~W}$

OGIER

Dane in vanguard of Charlemagne's army

$274 \mathrm{~W}$

OLIVER

Roland's friend, killed by Marganice

203W

OTHON

One of 12 peers, guarded French dead

$344 \mathrm{~W}$

ROLAND

Charlemagne's nephew, died at Roncevaux

030W

TURPIN

Archbishop of Rheims

$43 \mathrm{~N}$

000

REGIO

CASSINI

G.D. Cassini (1625-1712) discoverer of four satellites of Saturn

TERRA

RONCEVAUX the Spaniards 


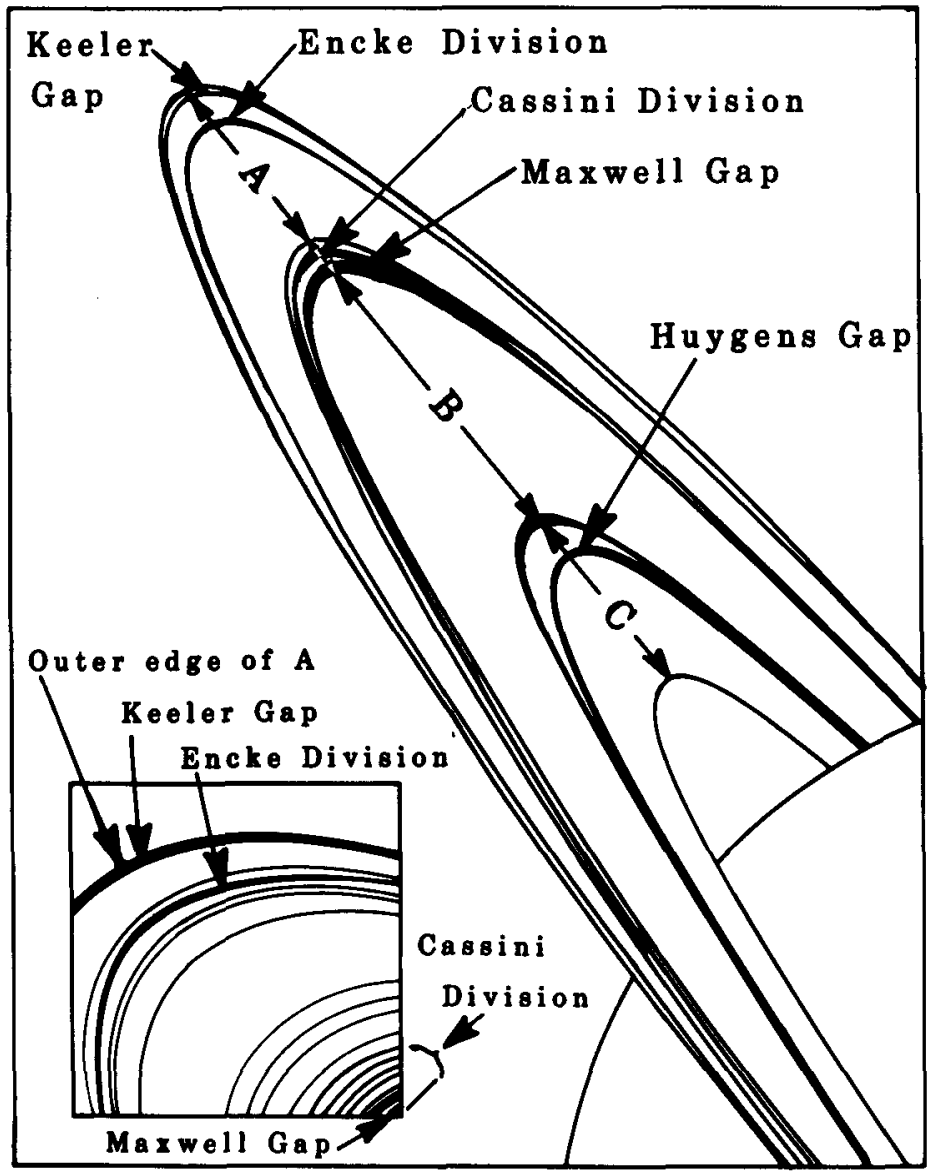

Saturn Ring Nomenclature 